\title{
I eat when I'm sad, I eat when I'm glad: on the role of cue reactivity and classical conditioning in emotional eating
}

Citation for published version (APA):

Bongers, P. M. H. (2015). I eat when I'm sad, I eat when I'm glad : on the role of cue reactivity and classical conditioning in emotional eating. [Doctoral Thesis, Maastricht University]. Maastricht University. https://doi.org/10.26481/dis.20151215pb

Document status and date:

Published: 01/01/2015

DOI:

10.26481/dis.20151215pb

Document Version:

Publisher's PDF, also known as Version of record

\section{Please check the document version of this publication:}

- A submitted manuscript is the version of the article upon submission and before peer-review. There can be important differences between the submitted version and the official published version of record.

People interested in the research are advised to contact the author for the final version of the publication, or visit the DOI to the publisher's website.

- The final author version and the galley proof are versions of the publication after peer review.

- The final published version features the final layout of the paper including the volume, issue and page numbers.

Link to publication

\footnotetext{
General rights rights.

- You may freely distribute the URL identifying the publication in the public portal. please follow below link for the End User Agreement:

www.umlib.nl/taverne-license

Take down policy

If you believe that this document breaches copyright please contact us at:

repository@maastrichtuniversity.nl

providing details and we will investigate your claim.
}

Copyright and moral rights for the publications made accessible in the public portal are retained by the authors and/or other copyright owners and it is a condition of accessing publications that users recognise and abide by the legal requirements associated with these

- Users may download and print one copy of any publication from the public portal for the purpose of private study or research.

- You may not further distribute the material or use it for any profit-making activity or commercial gain

If the publication is distributed under the terms of Article $25 \mathrm{fa}$ of the Dutch Copyright Act, indicated by the "Taverne" license above, 
I eat when I'm sad, I eat when I'm glad

On the role of cue reactivity and classical conditioning in emotional eating

Peggy Bongers 
I eat when I'm sad, I eat when I'm glad

On the role of cue reactivity and classical conditioning in emotional eating

Cover design: Jayce Yard - CLRD Graphic Design

Layout \& Printing: Ridderprint BV

ISBN: 978-94-6299-229-0

(c) Peggy MH Bongers, Maastricht 2015 


\section{I eat when I'm sad, I eat when I'm glad \\ On the role of cue reactivity and classical conditioning in emotional eating}

PROEFSCHRIFT

ter verkrijging van de graad van doctor aan de Universiteit Maastricht, op gezag van de Rector Magnificus, Prof. Dr. L. L. G. Soete, volgens het besluit van het College van Decanen,

in het openbaar te verdedigen op

dinsdag 15 december 2015 om 14.00 uur

door

Peggy Margaretha Henrica Bongers 


\section{Promotor}

Prof. Dr. A. T. M. Jansen

\section{Co-promotor}

Dr. R. C. Havermans

\section{Beoordelingscommissie}

Prof. Dr. M. L. Peters (voorzitter)

Prof. Dr. M. J.H. Huibers (Vrije Universiteit Amsterdam)

Dr. C. Martijn

Prof. Dr. D. T. D. de Ridder (Universiteit Utrecht)

Dr. T. Smeets 


\section{CONTENTS}

Chapter 1 General Introduction 7

Chapter 2 Happy eating: The underestimated role of overeating in a positive mood

Chapter 3 Happy eating: The Single Target Implicit Association Test predicts overeating after positive emotions

Chapter 4 'Emotional' does not even start to cover it: Generalization of overeating in emotional eaters

Chapter $\mathbf{5}$ Being impulsive and obese increases susceptibility to speeded detection of high-calorie foods

Chapter 6 Emotional eating and Pavlovian learning: Does negative mood facilitate appetitive conditioning?

Chapter 7 Emotional eating and Pavlovian learning: Evidence for conditioned appetitive responding to negative emotional states

Chapter 8 General Discussion

References

Summary

Samenvatting

Valorization Addendum

Dankwoord

Curriculum Vitae 

$1 \mid$

General Introduction 

Emma is a 21-year-old student. She is quite happy with her life, and things are going well. However, today she found out she failed an important exam for the second time, even though she studied very hard for it. She experiences a sense of desperation. She sits down on the couch with a big bucket of Ben \& Jerry's.

Louise (54 years old) has been separated from her husband for two months. Although she is slowly getting used to the idea, she sometimes still becomes overwhelmed by sadness and anger. To be prepared for these times, she makes sure to always have chocolate in the cupboard.

Thirty-two-year-old Stacey has a top position in the company she works for and this earns her a good salary. However, her job is stressful because of the responsibilities she carries and the strict deadlines she has to stick to. More and more often, she finds herself eating snack food when she is alone in her office.

The situations outlined above are all quintessential examples of emotional eating. Emotional eating, also known as comfort eating or stress eating, refers to eating in response to negative emotions. It is a behaviour generally believed to relieve negative affect (Ganley, 1989), or to temporarily escape from it (Heatherton \& Baumeister, 1991), although this has not been irrefutably demonstrated (Gibson, 2012; Haedt-Matt \& Keel, 2011). Emotional eating can be considered a form of overeating or eating in the absence of hunger, as generally no feelings of hunger or a physical necessity for food intake are present. Precisely because of this characteristic, emotional eating is a risk factor for overweight (defined as having a Body Mass Index (BMI) between 25 and 29.9, where BMI is $\mathrm{kg} / \mathrm{m}^{2}$ ) and obesity (BMI > 30). 
Obesity is a worldwide problem that continues to grow. An overview of the change in overweight and obesity rates in the Netherlands from 1981 to 2004 was reported by Schokker, Visscher, Nooyens, Van Baak, and Seidell (2007). The prevalence of overweight among male adults increased from 37\% (of which 4\% obese) in 1981 to 51\% (10\% obese) in 2004. For females, these numbers increased from 30\% (6\% obese) to $42 \%$ (12\% obese). More recent figures for 2012, as collected by CBS and RIVM (Health Monitor 2012), show prevalence rates of 53\% overweight (of which $11.3 \%$ obese) for males and $43.7 \%$ overweight (of which $13.9 \%$ obese) in females. In the US, recent overweight and obesity rates were reported to be as high as $71.6 \%$ and $33.7 \%$ for males, and $66.5 \%$ and 36.5\% for females, respectively (Ogden, Carroll, Kit, \& Flegal, 2014). Obesity has serious negative consequences not only for the well-being of obese individuals themselves, but also for society in general. Overweight and especially obesity have been shown to have adverse medical effects, such as increased risk of cardiovascular disease, type 2 diabetes, certain forms of cancer, and hypertension. In addition, obesity is the second leading cause of preventable death in the US (Stein \& Colditz, 2004; Wyatt, Winters, \& Dubbert, 2006). Furthermore, the stigma attached to obesity can lead to psychosocial problems in obese individuals, including being discriminated against, increased anxiety and depression, and low self-esteem (Carr \& Friedman, 2005; Puhl \& Heuer, 2009). Finally, the treatment of obesity and its comorbid illnesses, as well as indirect effects such as lower productivity and work absence, compose a significant economic impact, estimated at 117 billion dollars per year in the US alone (Stein \& Colditz, 2004; Wyatt et al., 2006).

Several studies have shown an association between emotional eating and weight gain or weight status. In both men and women, positive associations have been found between self-reported emotional eating scores and weight status, BMI, waist circumference, and percentage body fat (Konttinen, Silventoinen, Sarlio-Lähteenkorva, Männistö, \& Haukkala, 2010; Laitinen, Ek, \& Sovio, 2002; Péneau, Ménard, Méjean, Bellisle, \& Hercberg, 2013). In a retrospective study, emotional eating was found to be a significant predictor of weight gain over 20 years in a sample of women aged 55-65 years (Hays \& Roberts, 2008). A longitudinal study spanning two years (Koenders \& Van Strien, 2011) also found a significant positive association between emotional eating and increase in BMI. However, emotional eating did not predict weight gain during one year in a sample of female freshman students (Lowe et al., 2006).

Given the potential role that emotional eating may play in weight gain and the aetiology of overweight and obesity, it is of great importance to further investigate this type of eating. This dissertation aims at addressing some open questions regarding emotional eating, focusing in particular on the specific cues that elicit overconsumption in emotional 
eaters and the involvement of classical conditioning in emotional eating. Furthermore, some attention is given to the role of individual differences, specifically weight status and impulsivity, in cued responding. The introduction will start with an overview of survey and experimental studies that have investigated emotional eating. This leads to the conclusion that although not all studies find evidence for this behaviour, there certainly are individuals who overeat in response to negative emotions. The next paragraph discusses theories that have attempted to explain the development of emotional eating, and finds that both theory and evidence are limited. The final section introduces classical conditioning as an important mechanism in emotional eating.

\section{Emotions and eating: current state of affairs}

A widely used method to assess whether an individual is an emotional eater is through self-report questionnaires. Such questionnaires originated from early studies on the role of dietary restraint (i.e., the self-reported intention to deliberately restrict food intake in order to lose weight) in eating behaviour. These studies gave rise to the notion that measures of restraint should discriminate between individuals who are continuously successful in restricting their food intake, and individuals who tend to overeat or break their diet. As this discrimination was not present in the questionnaires used at the time (e.g., Restraint Scale; Herman \& Mack, 1975), new questionnaires were developed. In their Three-Factor Eating Questionnaire (TFEQ) Stunkard and Messick (1985) made a distinction between restrained and disinhibited eating, with the latter type of eating referring to overeating in response to internal (e.g., emotions) or external (e.g., the sight or smell of food) cues. A further distinction was made when the Dutch Eating Behaviour Questionnaire (DEBQ) was introduced (Van Strien, Frijters, Bergers, \& Defares, 1986), which consists of subscales to measure dietary restraint (DEBQ-DR), external eating (DEBQ-EX), and emotional eating (DEBQ-EE). The DEBQ-EE made it possible to single out emotional eaters specifically, regardless of any other characteristics (e.g., weight status, restraint) they might have. Additional self-report questionnaires were developed in later years (Emotional Eating Scale (EES), Arnow, Kenardy, \& Agras, 1995; Emotional Eating Scale-II (EES-II), Kenardy, Butler, Carter, \& Moor, 2003; Emotional Overeating Questionnaire (EOQ), Masheb \& Grilo, 2006; Emotional Appetite Questionnaire (EMAQ), Nolan, Halperin, \& Geliebter, 2010). Although the design of the questionnaires varies, what characterises all of them is the retrospective self-report of food intake in response to negative (TFEQ, DEBQ, EES, EOQ) and positive (EES-II, EMAQ) emotions.

The following two paragraphs provide an overview of survey and diary studies on emotional eating on the one hand, and experimental studies on the other hand. Some 
studies have used the aforementioned questionnaires to investigate eating behaviour of self-reported emotional and non-emotional eaters. Other studies do not incorporate such questionnaires but instead focus on how individual characteristics (e.g., gender, dietary restraint, weight status) affect emotional eating behaviour. Although the definition of emotional eating refers to overeating in response to specifically negative emotions, recent years have seen a limited attention to eating behaviour in positive moods as well.

\section{Survey and diary studies}

Epidemiological and survey studies have shown that women report themselves to be more likely to overeat during stress, while men report overeating when anxious or bored (Bennett, Greene, \& Schwartz-Barcott, 2013; Zellner et al., 2006). Both men and women have reported feeling more inclined to eat after imagining happy compared to sad events, although the preferred food differed across gender (Christensen \& Brooks, 2006). Kandiah, Yake, Jones, and Meyer (2006) found no differences in appetite reported by restrained and unrestrained eaters following stressful or non-stressful situations. Weinstein, Shide, and Rolls (1997) investigated the effects of dietary restraint and disinhibition on food intake after stress. For females, they found a positive relationship between disinhibition, but not restraint, and overeating in response to stress. For males, neither of the constructs was associated with stress-induced overeating. Finally, healthy-weight and overweight adolescent students did not differ in the relationship between perceived stress and self-reported emotional eating (Nguyen-Rodriguez, Chou, Unger, \& Spruijt-Metz, 2008).

Other studies have taken a diary approach, in which participants fill out eating-related questionnaires, and report their mood states, negative life events and food intake during a certain period of time. Compared to a non-stressful period, O'Connor and O'Connor (2004) found increased snacking among current dieters during times of stress, but only when these dieters were also low in conscientiousness. O'Connor, Jones, Conner, McMillan, and Ferguson (2008) found a positive relationship between the experience of daily hassles and intake of snacks high in fat and sugar, and this relationship was more pronounced in participants scoring high on emotional eating, dietary restraint, external eating and disinhibition, as well as in females and obese individuals. Another diary study also reported that an increase in number of hassles was associated with an increase in snacking, but only in individuals with high stress cortisol reactivity (Newman, O'Connor, \& Conner, 2007). In addition, they found that in the high compared to low cortisol reactors, emotional eating, dietary restraint, external eating and disinhibition were more strongly related to snacking, regardless of hassles. A study conducted during the aftermath of a destructive earthquake 
revealed that emotional eaters increased their food intake in the weeks after the event, but only when they also scored high on a measure of earthquake-related distress (Kuijer \& Boyce, 2012).

A number of other diary studies have reported different findings. Adriaanse, de Ridder, and Evers (2011) showed that not emotional eating, but habits and restraint were predictive of snacking behaviour. Conner, Fitter, and Fletcher (1999) identified only external eating as a significant moderator of the relationship between hassles and food intake, and found no effect of emotional eating. In a study specifically aimed at obese individuals, both emotional eating and external eating were positively related to consumption of foods with high sugar or fat content, but not to food intake in general. When taking mood into account, participants scoring either high or low on emotional eating did not differ in food intake in response to negative or positive events (Brogan \& Hevey, 2013). A diary study among obese and healthy-weight participants revealed no significant differences in eating in response to negative or positive emotions between groups (Lowe \& Fisher Jr, 1983). Another diary study among obese participants showed larger meals were consumed in both positive and negative as opposed to neutral situations (Patel \& Schlundt, 2001).

To summarize, survey and diary studies do not result in consistent findings regarding the relationship between negative emotional states and food intake. Although some studies find evidence for increased food intake during a negative emotional experience, this is often contingent on additional factors, such as cortisol reactivity or dietary restraint. A similar number of studies fail to demonstrate a relationship between emotional eating scores, emotions and eating.

It is important to consider the drawbacks of the aforementioned studies, which have relied solely on self-report. Answers to self-report questionnaires and filling out of selfreported food diaries are susceptible to intentional (e.g., socially desirable responding) and unintentional (e.g., forgetting) distortions. A 'triple recall bias' (Evers, de Ridder, \& Adriaanse, 2009) has been suggested to affect responses to self-report measures of emotional eating. Triple recall bias refers to the difficulties in recalling negative emotions, recalling food intake, and the association between the two. Although the issues regarding questionnaires remain, experimental studies can offer a more controlled environment for measuring food intake. Moreover, the design of survey and diary studies does not warrant conclusions regarding causality, a gap that can be solved by experimental designs. 


\section{Experimental studies}

Experimental studies investigating emotional eating generally follow a standard paradigm. In this paradigm, participants undergo a mood induction procedure aimed at eliciting a specific mood state. Upon completion of the mood induction, participants take part in a bogus taste test. While participants are under the impression that their food ratings and food preferences are examined, the actual measure of interest is food intake. Although similar in design, studies vary considerably in the specific emotions that are induced, as well as the way in which emotion induction is achieved. Table 1.1 provides an overview of the key experimental studies on emotional eating, outlining the groups, the induced moods, the applied mood induction paradigms and the main findings.

A number of studies have compared emotional eating in high vs. low scorers on emotional eating questionnaires. While the obvious prediction of emotional eating scales is that high scorers increase their food intake in negative emotional states compared to a control condition and compared to low scorers, findings on this matter are far from conclusive. Whereas some studies find clear evidence for increased intake in response to negative emotions in self-reported emotional eaters, others find no such evidence or report mixed results. Raspopow, Abizaid, Matheson, and Anisman (2014) measured food intake in anticipation of a stressful event (i.e., public speech), and found increased intake in emotional eaters compared to non-emotional eaters. Van Strien and colleagues (Van Strien, Cebolla, et al., 2013; Van Strien, Herman, Anschutz, Engels, \& de Weerth, 2012, study 2) reported increased food intake in emotional eaters in a negative compared to a neutral or positive mood and compared to non-emotional eaters, but only when exclusively extreme scorers on the DEBQ-EE (i.e., below the $20^{\text {th }}$ and above the $80^{\text {th }}$ percentile) were included. After reanalyzing data from one of these samples (Van Strien et al., 2012, study 2), blunted cortisol stress reactivity (Van Strien, Roelofs, \& de Weerth, 2013), an atypical hunger stress response (i.e., an increase in hunger during stress), and high inhibitory control (Van Strien, Ouwens, Engel, \& de Weerth, 2014) were found to moderate the relationship between negative mood and intake. However, even when only considering extreme scorers, another study (Van Strien et al., 2012, study 1) showed that although emotional eaters increased and non-emotional eaters decreased their food intake after negative emotions, both groups ultimately consumed the same amount of food in the negative mood.

No evidence for emotional eating was obtained by Kenardy et al. (2003), who compared intake in a negative mood to intake in a positive mood, and found decreased intake during a negative mood, with no effect of emotional eater status. Likewise, in four studies (Evers et al., 2009), no support was found for emotional eaters increasing their food intake in re- 


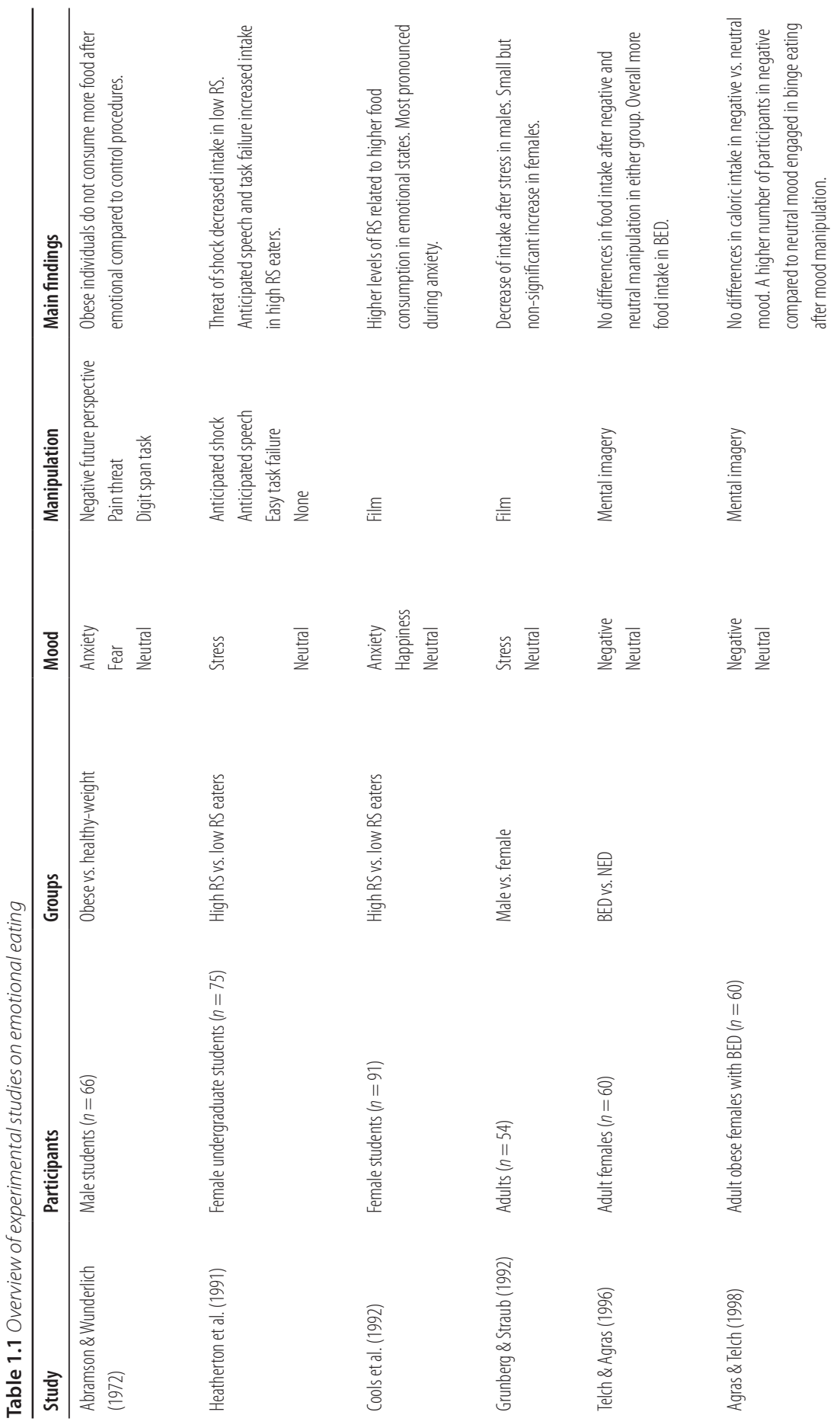




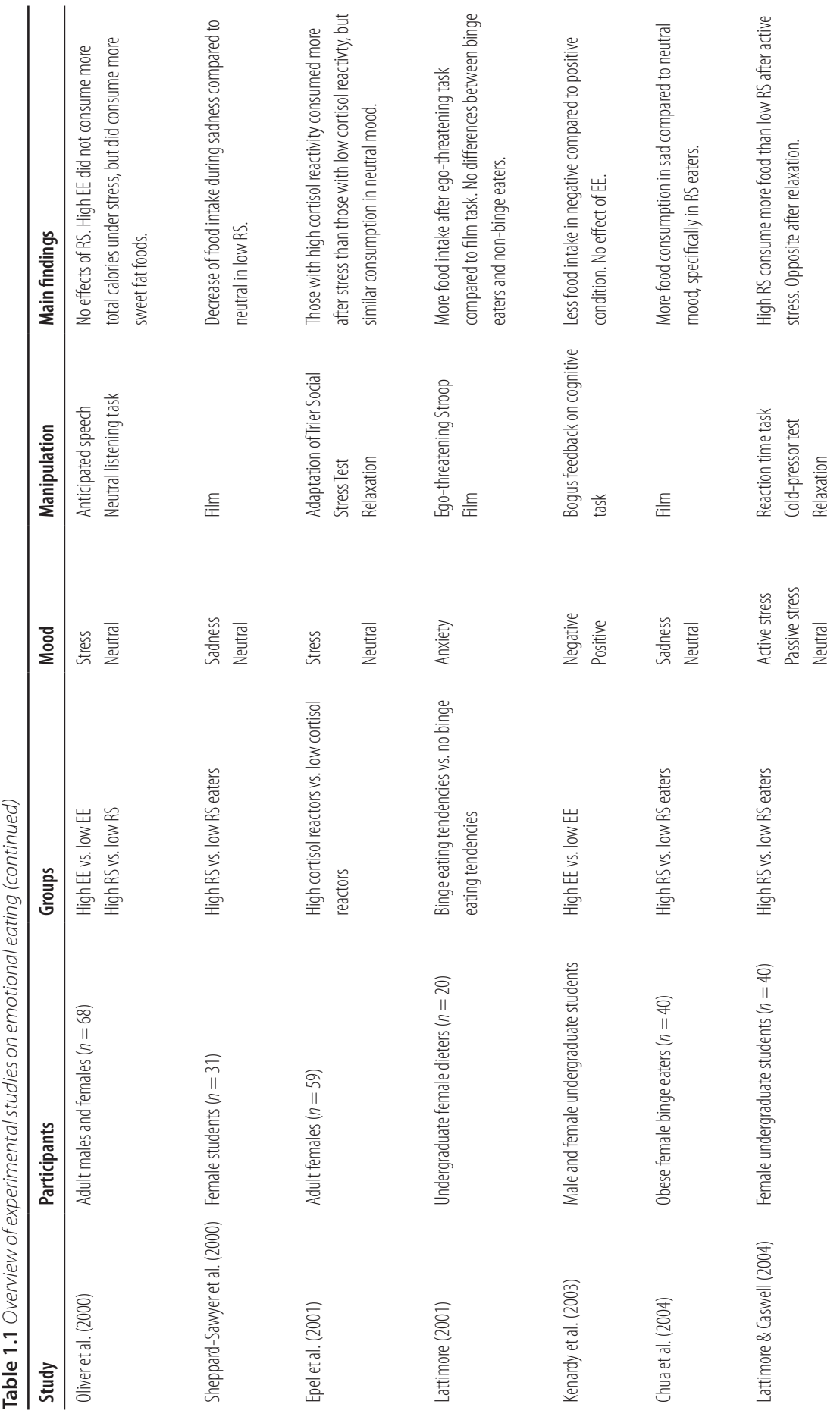




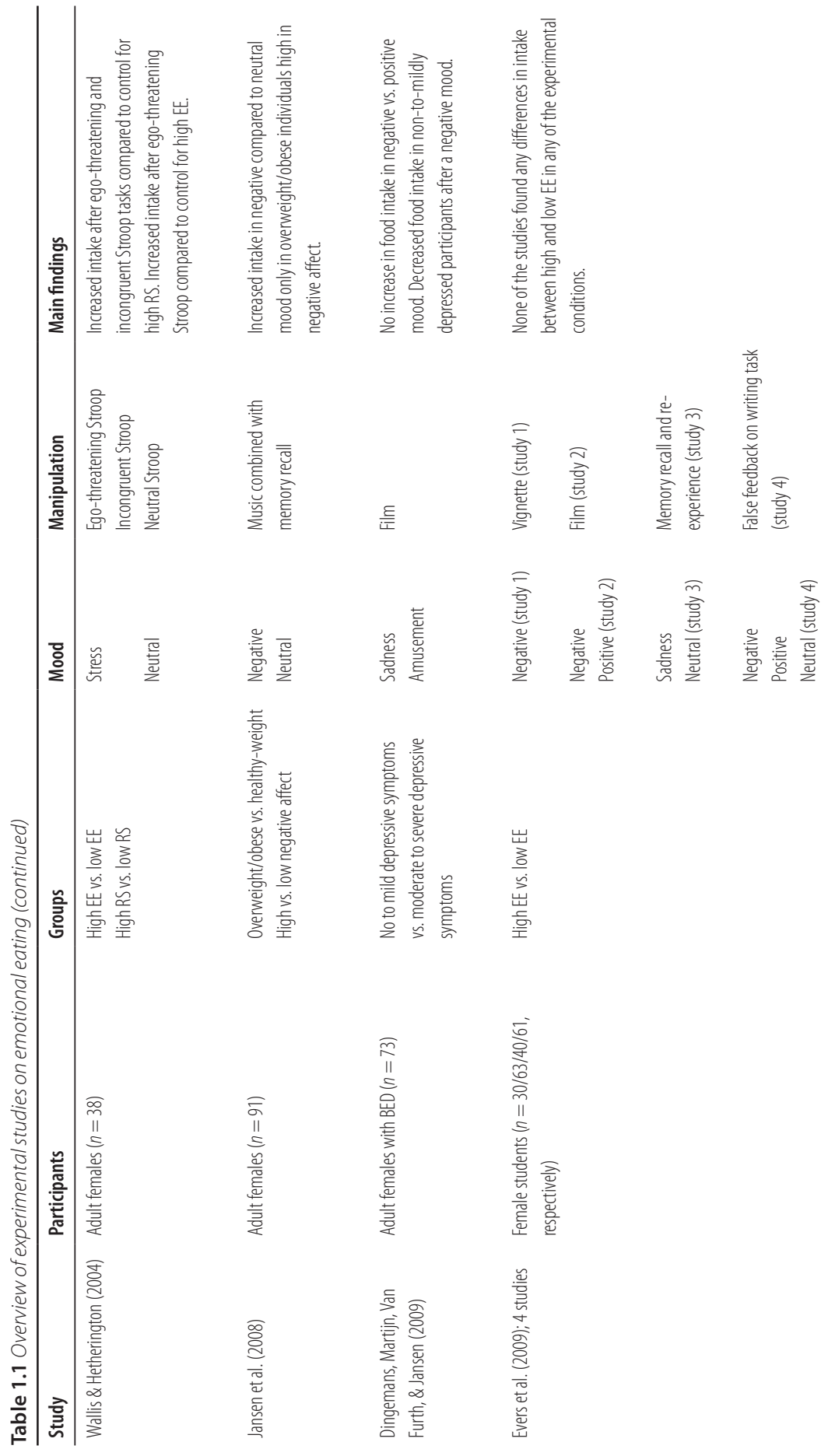



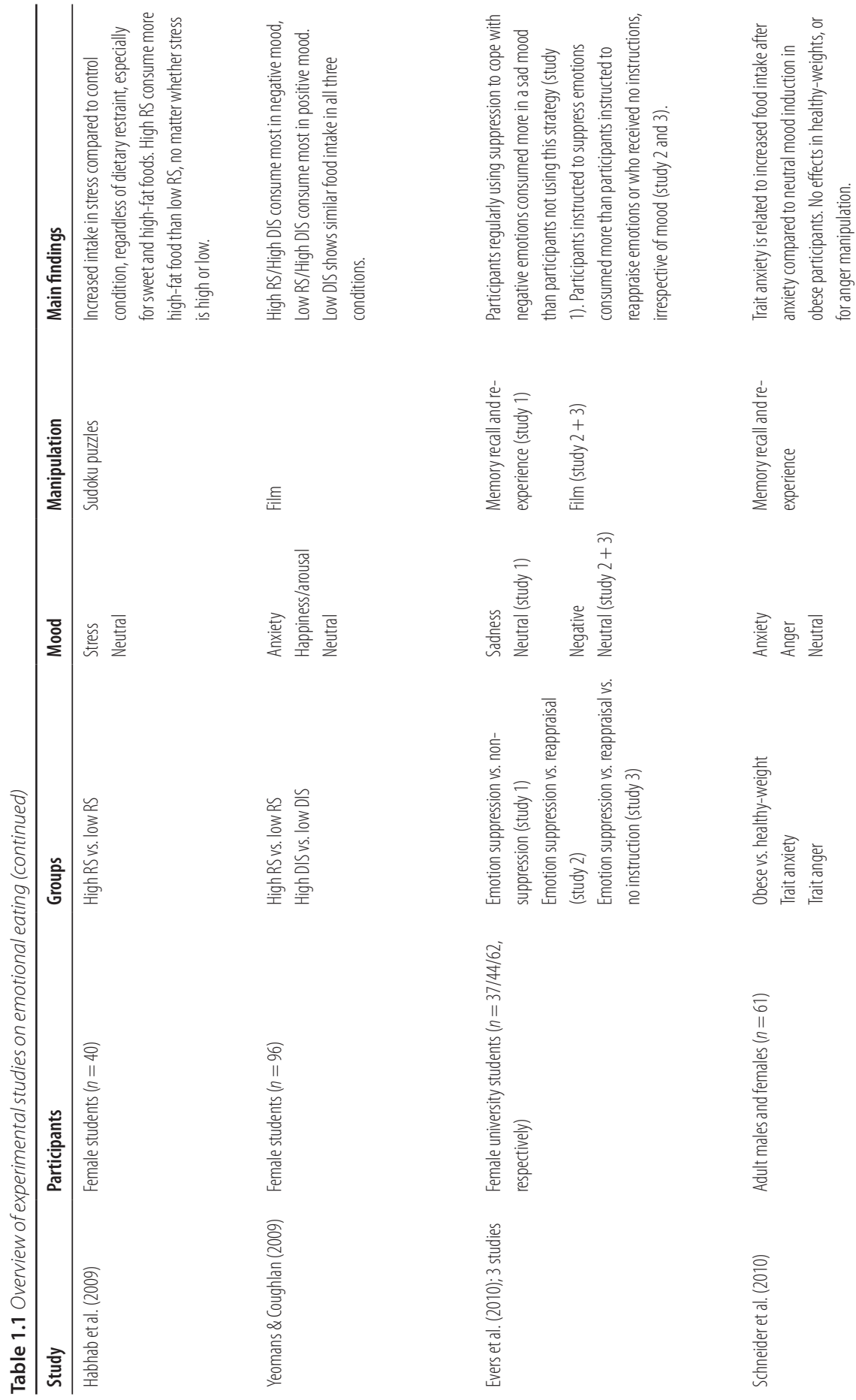


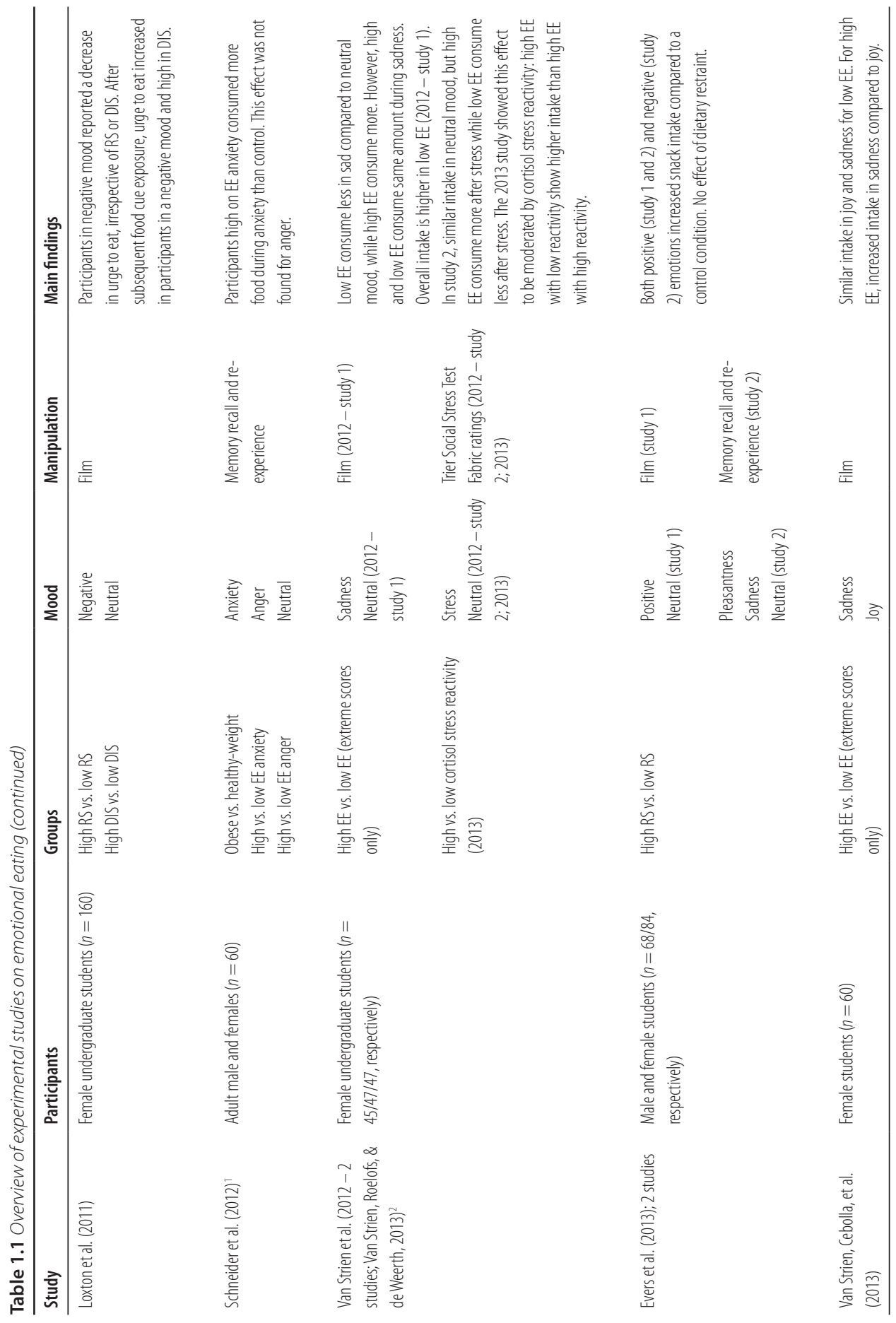




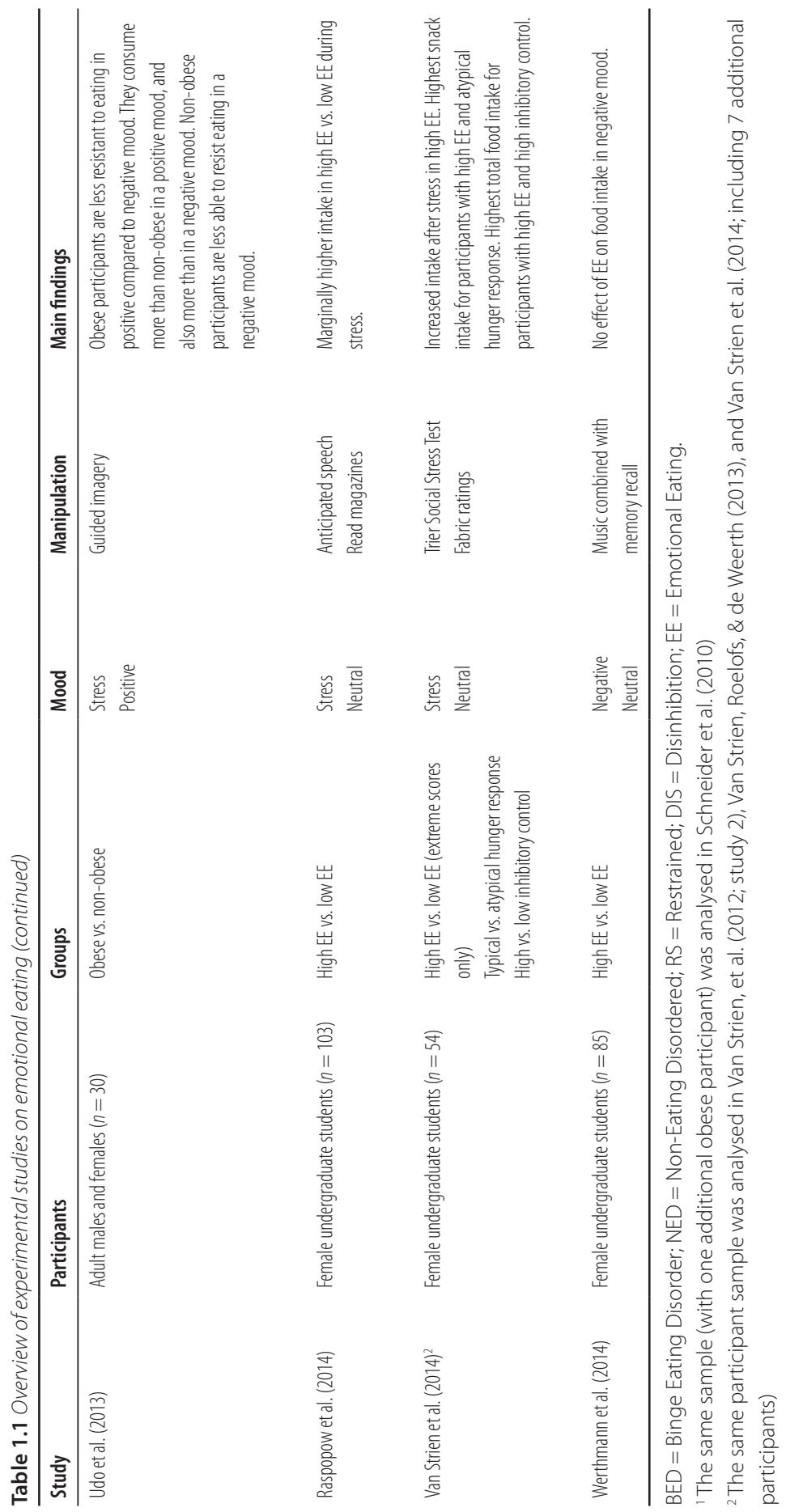


sponse to negative emotions, either compared to a control condition or to non-emotional eaters. Werthmann et al. (2014) were also unable to discover increased food intake in emotional eaters in a sad mood. Looking specifically at anger and anxiety, Schneider et al. (2012) compared food intake after inducing these emotions with a neutral control condition, and observed increased intake after an anxiety induction in participants scoring high on the EES anxiety subscale. However, no such effect was found for anger. Oliver, Wardle, and Gibson (2000) measured food intake during a buffet lunch. Although they did not find increased food consumption overall in stressed emotional eaters, this group did consume more sweet high-fat foods than non-stressed emotional eaters or non-emotional eaters. Using two different stressors, Wallis and Hetherington (2004) found greater intake in emotional eaters after an ego-threatening stress manipulation relative to a control procedure, but not after an equally stressful manipulation not involving ego-threat.

Finally, two studies investigated the effect of mood on food intake in disinhibited eaters (which includes both emotional and external eating), as measured by the TFEQ. Loxton, Dawe, and Cahill (2011) found a decrease in urge to eat following a negative mood, independent of disinhibition scores. However, upon subsequently being presented with food, urge to eat increased for women in a negative mood who were also high in disinhibition. Yeomans and Coughlan (2009) reported increased food intake in a negative mood for participants scoring high on both Restraint and Disinhibition subscales, and increased food intake in a positive mood for participants low on Restraint and high on Disinhibition. Participants with low disinhibition scores did not alter their food intake across conditions. However, for both studies it is impossible to determine whether the results were driven by emotional eating or external eating tendencies.

Taken together, experimental studies show that high scores on emotional eating scales are not necessarily predictive of increased food intake in a negative mood. When high and low scorers on emotional eating questionnaires (i.e., emotional and non-emotional eaters) are compared, only few studies find definite evidence for emotional eating. Most studies, however, do either not find any differences between emotional and non-emotional eaters concerning food intake, or only under specific conditions.

Other studies have investigated food intake during a negative mood without including scores on self-report emotional eating scales. A number of these studies are consistent in their finding that high levels of dietary restraint are related to increased food intake after experiencing negative emotions, while individuals low in restraint show either no change or a decrease in food intake (Cools, Schotte, \& McNally, 1992; Heatherton, Herman, \& Polivy, 1991; Lattimore \& Caswell, 2004; Sheppard-Sawyer, McNally, \& Fischer, 2000), although slight 
differences can emerge depending on the type of manipulation used. However, Habhab, Sheldon, and Loeb (2009) did not find an interactive effect of stress and dietary restraint. They reported higher food intake in restrained vs. unrestrained women, as well as a preference for sweet and high-fat food in stressed vs. non-stressed women. Similarly, Evers, Adriaanse, de Ridder, and De Witt Huberts (2013) reported overeating in positive and negative moods compared to neutral moods in both restrained and unrestrained eaters. In addition to dietary restraint, high cortisol reactivity (Epel, Lapidus, McEwen, \& Brownell, 2001) and a suppressive emotional coping style (Evers, Stok, \& de Ridder, 2010) have been proposed as risk factors for overeating in response to negative emotions. Highlighting the possible role of gender, Grunberg and Straub (1992) found a considerable decrease of food intake in stressed men, but a slight though insignificant increase in stressed women.

Attention has also been given to the role of weight status and Binge Eating Disorder (BED) in relation to emotional eating, yielding mixed results. Assessing resistance to commence eating during negative emotions, Udo et al. (2013) found healthy-weight participants to be less resistant to eating (i.e., shorter latencies until deciding to start eating) in a negative compared to a positive mood. However, obese individuals showed less resistance to eat in response to positive emotions, and ultimately consumed more calories than healthyweight participants or when compared to the negative mood condition. In contrast, Jansen et al. (2008) showed increased food intake in obese individuals after negative emotions, but only when these individuals were also high in negative affect. Similarly, Schneider, Appelhans, Whited, Oleski, and Pagoto (2010) found increased food intake in an obese sample after an anxiety induction, but only in the individuals high in trait anxiety. No such effects were obtained for anger. In both studies, obese participants with low trait anxiety or low negative affect did not differ from healthy-weight controls. In an older study, no evidence was found for increased intake during fear or anxiety in obese male students (Abramson \& Wunderlich, 1972). Chua, Touyz, and Hill (2004) reported that obese individuals with BED showed higher intake in a negative compared to a neutral mood. In contrast, other studies revealed no effects of mood on food intake in a similar obese sample (Agras \& Telch, 1998; Telch \& Agras, 1996). However, while BED-patients were found to generally consume more food than participants without the disorder in one study (Telch \& Agras, 1996), another study (Lattimore, 2001) showed no such differences between students with high and low binge-eating tendencies. Both groups consumed more food after a ego-threatening relative to a non-ego-threatening anxiety task. Comparing food intake in depressed and nondepressed obese BED-patients, Dingemans, Martijn, Van Furth, and Jansen (2009) found 
non-depressed participants to eat less in a negative than a positive mood, and also less than depressed patients under both mood conditions.

To summarize, as with the experimental studies that differentiate between emotional and non-emotional eaters, results of studies on emotion-induced eating behaviour are mixed. There is no unequivocal evidence for increased intake after negative emotions in a specific group of individuals or under specific circumstances.

\section{Emotional eating explained}

Despite the varied findings as outlined above, there clearly are certain individuals who increase their food intake when feeling negative. This is an interesting observation, as the normal response to negative or stressful situations is to reduce food intake (Wardle, 1990). The usual response to (physiological) stress is a decrease in gut activity, which in turn decreases the need to eat. This raises the question as to why some individuals behave differently and how this increase in food intake in the face of adversity develops. Explanations for emotional eating were first provided in the late 1950 s and early 1960s, when emotional eating was introduced as an explanation for obesity. Based on clinical observations, psychosomatic theory (Bruch, 1964; Kaplan \& Kaplan, 1957) states that a correct interpretation of bodily states needs to be learned, and that incorrect learning is responsible for emotional eating. Individuals affected by emotional eating have never learned to differentiate emotional states from hunger states, likely due to parental feeding practices. For example, an individual who as a child was always given food as a pacifier, no matter what discomfort was burdening him (e.g., hunger, sadness, pain), will also later in life continue to turn to food in times of distress. Thus, whereas negative emotions such as stress or sadness lead to a decrease in gastric contractions and subsequent food intake in most people, this was thought to have no effect on food intake in the obese.

Based on a lack of support for the psychosomatic theory (i.e., no evidence for increased food intake during emotional states in obese individuals), Schachter (Schachter, 1968; Schachter, Goldman, \& Gordon, 1968) proposed the externality theory of obesity, which postulates that obese individuals indeed have difficulties understanding internal cues (such as gastric contractions) that signal hunger, but in contrast to psychosomatic theory, do not respond to such cues. Instead, food intake in the obese is considered to be controlled by external cues, such as the sight and smell of food, time of day, and eating behaviour of others. Although Schachter reported several studies in favour of externality theory (for an overview see Schachter, 1968), the results have also been criticized (Milich, 1975). 
Observations that not all obese individuals overeat in response to external cues (Nisbett, 1968) prompted the idea that overeating may not be dependent on weight status, but on other individual differences. Taking dietary restraint into account as one such individual difference, Restraint Theory (Herman \& Mack, 1975) argues that restrained eaters are generally successful in controlling their food intake, but that certain challenging events or situations, including the presence of palatable food or the experience of negative emotions, can lead to a lessening of control and unintended food consumption. Restraint Theory emphasized the role of dietary restraint as a risk factor for emotional and external eating, thereby moving away from the idea that these eating behaviours are only present in the obese population.

It is somewhat surprising that explanations for emotional eating have rarely been tested. Instead, the lion's share of emotional eating studies is descriptive in nature. They describe eating behaviour in response to negative emotions in individuals who are for example obese, binge eaters, or score high on self-report measures of dietary restraint or emotional eating. However, studies on how emotional eating develops and is maintained are scarce. Somewhat in line with psychosomatic theory (Bruch, 1964), a few studies have focused on parental feeding strategies and emotional eating in young children. These studies do not necessarily assume that emotional eaters cannot distinguish between hunger and emotions, but rather that they have learned to use food to cope with unpleasant feelings. In a sample of 2-year old children, it was reported that mothers who were emotional eaters were more likely to use food to soothe their child in times of emotional need, which in turn was related to emotional eating in the child (Rodgers et al., 2013). Similar findings were obtained in children aged 8 to 12 (Braden et al., 2014). In an experimental design, it was found that 3-5-year old children whose mothers regularly used food to regulate their child's emotions consumed more chocolate in a negative compared to a neutral mood (Blissett, Haycraft, \& Farrow, 2010). Other studies have found specific parenting styles and emotional responsiveness to be predictors of emotional eating in children and adolescents (Snoek, Engels, Janssens, \& Van Strien, 2007; Topham et al., 2011).

A key factor in Bruch's theorizing and the aforementioned children studies appears to be learning: children learn that the appropriate response to feeling bad is eating, or that eating when feeling bad helps to regulate emotions. It is also conceivable that individuals learn that palatable food offers escape from or alleviation of negative affect at a later age. This learning aspect of emotional eating has led researchers to suggest that classical conditioning is involved in emotional eating (Greeno \& Wing, 1994; Jansen, 1990; Jansen, Havermans, \& Nederkoorn, 2011; Macht, 2008; Wardle, 1990). 


\section{A classical conditioning perspective on emotional eating}

In terms of classical conditioning, food intake is an unconditioned stimulus (US): a stimulus that unconditionally and automatically elicits a response. These automatic or unconditioned responses (URs) to food are the metabolic responses. When food intake is consistently and exclusively preceded by specific cues, these cues can develop into conditioned stimuli (CSs) that have the ability to predict food intake and elicit responses (conditioned responses; CRs) that prepare the body for food intake. Such responses are termed cephalic phase responses and include for example changes in heart rate, salivation, blood pressure, skin conductance, insulin release and blood sugar (Mattes, 1997; Nederkoorn, Smulders, \& Jansen, 2000). Subjectively, cephalic phase responses are experienced as food craving (Jansen, 1998; Nederkoorn et al., 2000). In the context of classical conditioning, all physiological and psychological conditioned responding is labelled as cue reactivity. In case of appetitive behaviours, cue reactivity encompasses the aforementioned physiological responses (e.g., increase in salivation and release of insulin) and the accompanying craving, but also for example brain activation patterns, attention bias for food, approach tendencies, and actual food intake.

Palatable high-calorie foods have high potential for conditioning. When food intake occurs, any cue or context present at that time can become associated with eating (Bouton, 2011; Bouton, Woods, Moody, Sunsay, \& García-Gutiérrez, 2006). The possibilities for such associations are seemingly endless (Bouton, 2011): food smells and tastes, time of day, food preparation rituals, the place where one eats and the tune of a television show are mere examples of potential signals for eating and classical conditioning. Animal studies have convincingly shown that previously neutral cues that have come to be predictive of food intake can elicit physiological responses to eating, such as salivation, release of insulin and increase in blood sugar (Bouton et al., 2006; Jansen, Havermans, \& Nederkoorn, 2011). Rats have been observed to increase intake when contextual cues are present that are associated with palatable food, even under conditions of satiety (Boggiano, Dorsey, Thomas, \& Murdaugh, 2009). Likewise, from classical conditioning studies in humans it becomes apparent that cued eating desires and cued overeating are relatively easily learned. Indeed, several studies have shown that neutral external cues can develop into appetitive conditioned stimuli, capable of predicting food intake. In such studies, a neutral cue (for example a green tray; called (S+) is repeatedly paired with intake of a palatable food, while a similar but not identical cue (white tray; CS-) is not paired with eating. Upon each CS presentation, participants are asked to rate their expectancy and desire to eat. Other outcome measures vary across studies, but include salivation, approach tendencies, and actual food intake. 
Of interest is whether after several (usually 4 or 5 for the CS+ and similar for the CS-) of these pairings, presented in a random order, participants respond differently to the CS+ compared to the CS-, which would indicate successful conditioning.

In a line of studies, Van Gucht and colleagues (Van Gucht, Baeyens, Hermans, \& Beckers, 2013; Van Gucht, Baeyens, Vansteenwegen, Hermans, \& Beckers, 2010; Van Gucht, Vansteenwegen, Beckers, \& Van Den Bergh, 2008; Van Gucht, Vansteenwegen, Van den Bergh, \& Beckers, 2008) used a rectangular white and a round green serving tray as neutral cues, and chocolate as a US. They demonstrated appetitive conditioning in all studies, with participants reporting higher expectancy to eat, higher desire to eat, and stronger automatic approach tendencies in response to the CS+ than the CS-. These results were replicated by Papachristou, Nederkoorn, Beunen, and Jansen (2013). Successful appetitive conditioning was also shown in response to other neutral cues, such as jewellery boxes (Van den Akker, Havermans, Bouton, \& Jansen, 2014; Van den Akker, Havermans, \& Jansen, 2015).

Other studies have looked into the role of context in appetitive conditioning. In a study among preschool children, Birch, McPhee, Sullivan, and Johnson (1989) showed contextual conditioning of meal initiation, with children showing significantly shorter latencies to start eating in the CS+ compared to the CS- environment. More recently, Van den Akker, Jansen, Frentz, and Havermans (2013) found differential responding to CS+ vs. CS- contexts (i.e., a museum and a dojo) in a virtual reality study. Participants reported increased expectancy and desire to eat in the CS+ compared to the CS- context, and showed increased salivation in response to the CS+ compared to baseline. Participants were also found to increase their actual food intake in the CS+ compared to the CS-context, but only when they scored high in self-reported impulsivity.

Impulsivity has been implicated in learning, cue reactivity and obesity. With regard to learning, Gray (Corr, Pickering, \& Gray, 1995) proposed in his BIS/BAS theory that individuals with a strong BAS (i.e., Behavioural Activation System; related to impulsivity) would show facilitated appetitive conditioning, while individuals with a strong BIS (i.e., Behavioural Inhibition System; related to anxiety) would show increased aversive learning. It is argued that the appearance of food (US) following a CS stimulates BAS, which leads to arousal. This in turn strengthens the association between that particular CS and US, and thus facilitates learning. Studies investigating this claim show mixed results however (Corr, 2004; Corr et al., 1995; Paisey \& Mangan, 1988; Zinbarg \& Mohlman, 1998; Zinbarg \& Revelle, 1989), and only two studies employed food as the appetitive stimulus (Papachristou et al., 2013; Van den Akker et al., 2013). With respect to cue reactivity, a number of studies have shown a positive relationship between impulsivity and cue-reactive responding to food in terms of 
desire to eat, desired portion size, attention bias and actual intake (see for example Guerrieri, Nederkoorn, \& Jansen, 2008; Guerrieri, Nederkoorn, Schrooten, Martijn, \& Jansen, 2009; Hou et al., 2011; Tetley, Brunstrom, \& Griffiths, 2010). Cue reactivity studies in smokers have provided evidence for increased physiological reactivity in individuals high in impulsivity (Doran, McChargue, \& Spring, 2008). Similarly, higher levels of impulsivity are associated with (Galanti, Gluck, \& Geliebter, 2007; Guerrieri, Nederkoorn, \& Jansen, 2007) - and have even been found to lead to (Guerrieri et al., 2009; Guerrieri, Nederkoorn, Stankiewicz, et al., 2007; Jansen et al., 2009) - increased food intake. Finally, impulsivity has also been found to be positively related to obesity (Guerrieri et al., 2008; Mobbs, Crépin, Thiéry, Golay, \& Van der Linden, 2010; Nederkoorn, Smulders, Havermans, Roefs, \& Jansen, 2006; Rydén et al., 2003) To summarize, impulsivity could influence the ease with which appetitive learning takes place, as well as the specific physiological and psychological behaviours resulting from this learning.

The studies discussed in this section consistently provide evidence for appetitive conditioning to external cues and contexts in humans. Similarly, internal cues such as cognitions and mood states have been suggested to have the potential to grow into conditioned stimuli for overeating (Greeno \&Wing, 1994; Jansen, 1998; Jansen, Havermans, \& Nederkoorn, 2011; Macht, 2008; Wardle, 1990). However, this has never been tested. With regard to emotional eating, it is of particular interest whether specific mood states can develop into conditioned stimuli predictive of food intake. To give a more concrete example, imagine an individual who experiences feelings of sadness, and eats a piece of chocolate. Sometime later, this individual feels sad again, and again consumes chocolate. When this continues over time, and the sadness and the chocolate consumption occur together repeatedly, at one point mere feelings of sadness will start to elicit cue reactivity; sadness has become a CS for chocolate intake. Thus, if moods are systematically associated with eating, they could eventually elicit desire to eat and actual overeating, and as such explain the origin or maintenance of emotional eating.

\section{Outline of this dissertation}

The primary aim of this thesis is to gain insight into open questions concerning emotional eating. More specifically, the main focus is on (1) the type of cues that elicit overeating in emotional eaters (chapters 2, 3 and 4), and (2) whether classical conditioning processes are involved in emotional eating (chapters 6 and 7). Attention is also given to (3) the role of individual differences in cued responding (chapter 5). Six studies have been conducted which addressed these three questions. 
Chapter $\mathbf{2}$ describes a study which investigated food intake of emotional eaters in response to negative, neutral, and positive emotions. Unlike negative emotions, positive emotions have not received a large amount of attention in the emotional eating literature, and only one study so far included a positive and negative condition, as well as a control condition. A secondary aim of this study was to look into the association between caloric intake and mood improvement. Participants viewed a positive, negative or neutral film clip to induce a corresponding mood. This was followed by a bogus taste test to measure food intake, during which participants rated their mood a number of times. It was hypothesized that emotional eaters would consume more food in the negative and positive mood compared to the neutral mood, as well as compared to the non-emotional eaters in the emotional conditions. With regard to the second aim, it was expected that both emotional and non-emotional eaters would report an increase in mood after consuming palatable food, but that this effect would be more pronounced in the emotional eaters.

Chapter $\mathbf{3}$ focuses on a study that was also on overeating in negative, positive and neutral moods, but that additionally dealt with some issues that have arisen with regard to the validity of emotional eating questionnaires. The study included two Single Target Implicit Association Tests (ST-IATS) in addition to the generally used self-report questionnaire. The ST-IATs are assumed to be more objective and implicit measures of emotional eating by measuring associations between negative emotions and food and between positive emotions and food. Furthermore, food intake was measured both during and after the mood manipulation. Participants viewed a positive, negative or neutral film clip to induce the corresponding mood. It was expected that the ST-IATs would be better at distinguishing emotional from non-emotional eaters than the questionnaire. Participants with strong food-negative associations were expected to overeat after negative emotions, while those with strong food-positive associations were thought to overeat after positive emotions. No effect of mood induction on food intake was expected for participants with weak associations.

Chapter 4 discusses a study that assessed food intake in emotional eaters not only during emotional states, but also after food cue exposure and a control condition. The study also questioned the validity of using different labels (i.e., emotional, external, restrained) to identify specific types of eaters, as opposed to making a broader and simpler distinction of cue-reactive vs. non-cue-reactive eaters. A behavioural measure of emotional eating was employed in addition to a self-report questionnaire. Participants were exposed to three experimental situations (experience of positive emotions, negative emotions, and exposure to food) as well as a control situation, which were all followed by a bogus taste test. As- 
suming that the labels are not valid, it was expected that emotional eaters would consume more food in all experimental conditions compared to the control condition, and more than non-emotional eaters in specifically the experimental conditions.

Chapter $\mathbf{5}$ describes a study on the relationship between cue reactivity, impulsivity and weight status. Obese and healthy-weight individuals were compared on attention bias for food measured by a Visual Search Task, while taking trait food craving and behavioural and self-report measures of impulsivity into account. It was predicted that higher levels of trait food craving and higher levels of impulsivity were related to a stronger attention bias for food, and that this relationship was most pronounced in obese participants.

Chapter 6 focuses on the role of classical conditioning in emotional eating. One proposed pathway is that, because of the rewarding properties of palatable food, individuals are faster to learn an association between a neutral cue and food when they are in negative mood compared to a neutral mood. Participants were brought into either a negative or neutral mood. They were then presented with two similar, but not identical, neutral cues of which one was repeatedly paired with food intake (CS+), while the other one was not (CS-). Expectancy to eat, desire to eat, salivation and food intake were measured, and it was hypothesized that although participants in both conditions would show appetitive conditioning (i.e., stronger expectancies and desires, more salivation, and more food intake in response to the CS+ compared to the (S-), this would be stronger in the negative mood condition. In addition, the role of impulsivity in appetitive conditioning was explored.

Chapter 7 tests another proposed pathway concerning classical conditioning and emotional eating; one in which negative emotions develop into CSs and over time start to predict food intake. For half the participants a variety of negative mood inducing cues (CS+) were repeatedly paired with food intake while neutral cues (CS-) were not. For the other half, neutral cues were paired with chocolate while negative cues were not. Participants reported expectancy and desire to eat after every trial and salivation measurements were taken at baseline and after conditioning. After the conditioning procedure all participants once more were brought into a negative mood and participated in a chocolate vs. money choice task. It was hypothesized that negative emotions could develop into reliable predictors of food intake, as shown by increased expectancy, desire, salivation and a preference for chocolate over money in response to the CS+ vs. CS- cue. This effect was expected to be stronger when negative cues compared to neutral cues were the CS+.

Chapter $\mathbf{8}$ provides a general overview of the studies and an integrative discussion of the results. In addition, the chapter provides methodological considerations, implications of the results for clinical practice, and suggestions for future research. 

2

\section{Happy eating: The underestimated role of overeating in a positive mood}

Published as:

Bongers, P., Jansen, A., Havermans, R., Roefs, A., \& Nederkoorn, C. (2013) Happy eating: The underestimated role of overeating in a positive mood. Appetite, 67, 74-80. 


\section{ABSTRACT}

Emotional eaters are often presumed to eat in response to negative emotions, while positive emotions have been largely neglected. The current study induced a positive, negative, or neutral mood in a student sample and subsequently measured food intake. In addition, the relation between caloric intake and mood improvement was assessed. It was expected that emotional eaters would consume more food than non-emotional eaters in the emotional conditions, and also more than in the neutral condition. Moderated regression analyses indeed showed a significant increase in food intake for emotional eaters in the positive compared to the neutral condition, and a trend towards higher food consumption than non-emotional eaters. No effects were found in the negative condition. With regard to mood changes during food intake, Pearson correlations demonstrated an association between amount of calories consumed and mood improvement after five minutes of food consumption. However, there was no evidence for differences between emotional and non-emotional eaters. The current findings underline the importance of positive emotions in emotional eating, and provide new insights on the relationship between eating and mood melioration.

Keywords: Emotional eating; Mood induction; Positive emotions; Food consumption; Mood repair. 


\section{INTRODUCTION}

Emotional eaters report to overeat in response to negative emotions, and are therefore considered to be at risk for becoming overweight or even obese (Hays \& Roberts, 2008). The concept of emotional eating originated several decades ago already (Bruch, 1964; Kaplan \& Kaplan, 1957; Schachter, Goldman, \& Gordon, 1968), and questionnaires developed to measure emotional eating (e.g. Dutch Eating Behaviour Questionnaire (DEBQ) (Van Strien, Frijters, Bergers, \& Defares, 1986; Van Strien, 2005); Emotional Eating Scale (EES) (Arnow, Kenardy, \& Agras, 1995); Emotional Overeating Questionnaire (EOQ) (Masheb \& Grilo, 2006)) have been around for the past 25 years as well. However, until quite recently, researchers rarely distinguished their participants on being classified as emotional or non-emotional eaters (that is, emotional eaters are thought to increase their food intake in response to negative emotions, while non-emotional eaters will not change their consumption levels, or might even restrict intake), even though it is important to gain knowledge on how exactly emotions influence self-reported emotional eaters.

With regard to negative emotions, Oliver, Wardle, and Gibson (2000) manipulated stress levels in their participants and presented them with sweet, salty and bland food, either high or low in caloric content. They found that emotional eaters in the stress condition consumed more sweet high-caloric foods than non-emotional eaters and participants in a non-stress condition. However, stress did not change overall food consumption. Evers, de Ridder, and Adriaanse (2009) showed in two experiments that participants scoring high on self-reported emotional eating did not consume more food after being brought in a negative emotional state than participants who scored low on emotional eating, nor did they increase their food intake in the emotional compared to the neutral condition. Adriaanse, de Ridder, and Evers (2011) found no influence of self-reported negative emotional eating on snacking in a diary-study.

In contrast to the previously described studies in which a negative mood was manipulated, Turner, Luszczynska, Warner, and Schwarzer (2010) induced a positive mood in emotional and non-emotional eaters, controlling for uncontrolled eating styles (i.e., difficulties with refraining from eating when food cues are presented). They found that food intake was not directly affected by emotional eating status. Finally, two studies induced both positive and negative moods in their participants. Kenardy, Butler, Carter, and Moor (2003) did not find any evidence for emotional eating after positive or negative mood induction, but did find lower food consumption in a negative compared to a positive mood, regardless of emotional eater status. Likewise, Evers et al. (2009) discovered no effects of either positive 
or negative emotions on food intake: emotional eaters did not consume more food than non-emotional eaters after any of the mood manipulations, nor did they have higher food intake after the emotional conditions compared to a neutral control condition.

Only this last study (Evers et al., 2009) manipulated both positive and negative mood, and included a control condition. Mood was manipulated by providing negative or positive feedback on the participant's performance on a task, thus being very personal and, in the negative condition, ego-threatening. Interestingly, studies in restrained eaters and dieters have shown that these specific groups of people actually tend to overeat when being in an ego-threatening situation, even more so than in other negative situations, like watching frightening movies (Heatherton, Herman, \& Polivy, 1991; Lattimore, 2001; Wallis \& Hetherington, 2004). However, it appears from the previously discussed studies (Evers et al., 2009; Kenardy et al., 2003; Oliver et al., 2000) that this was not the case in emotional eaters. Therefore, in the current study, a non-threatening mood manipulation was applied. Another reason for some of the null-findings in previous studies could be that participants were offered only salted crackers (i.e. Kenardy et al. (2003)), while some studies suggests that sweet high-fat foods are the preferred choice for emotional eaters (Konttinen, Männistö, Sarlio-Lähteenkorva, Silventoinen, \& Haukkala, 2010; Oliver et al., 2000).

Another issue concerns why people overeat in response to emotions. In (sub) clinical populations, such as binge eaters, affect regulation theories have been proposed to explain excessive food intake in response to emotions. These theories state that people overeat to reduce the affect they are experiencing, especially when negative (Ganley, 1989; Heatherton \& Baumeister, 1991). It is quite imaginable that comparable processes might be working in emotional eaters. Macht and Mueller (2007) examined the effects of chocolate consumption after experimentally inducing negative mood, and found that mood became significantly more positive in emotional eaters compared to non-emotional eaters, although the latter group also experienced a mood improvement. After both negative and positive emotion inductions, participants in a study by Kenardy et al. (2003) decreased in negative affect after eating. However, no differences were found between emotional and non-emotional eaters. Although increased mood after eating has been established by these studies, it is still unknown whether the amount of food consumed is of importance in reaching this improvement.

The present research aimed to investigate a reciprocal model of mood change and food intake in emotional eaters, in which mood change leads to increased food intake, and food intake in turn leads to positive changes in mood. We studied the effect of negative and positive emotional states on caloric food intake in self-reported emotional eaters by 
employing a non-ego-threatening mood manipulation. Positive emotions were included to incorporate suggestions from the clinical field that people report overeating after positive emotions, as well as to replicate previous studies (e.g. Evers et al., 2009; Kenardy et al., 2003). We hypothesised that after both negative and positive emotions, emotional eaters would consume more calories than non-emotional eaters, and that their caloric intake would be higher in both emotional conditions compared to the control condition. A second aim of the study was to shed more light on the relationship between food intake and mood change in emotional and non-emotional eaters. We hypothesised that there is a positive association between caloric intake and mood, most pronounced in emotional eaters.

\section{METHOD}

\section{Participants}

Eighty-seven (65 female) psychology students, enrolled in their second year at Maastricht University, participated in this study in return for course credit. One participant was excluded due to giving only extreme and inconsistent answers on several questionnaires. Age varied between 19 and 43 years $(M=21.6, S D=2.8$ ). The study was approved by the Maastricht University Ethical Committee.

\section{Measures}

Positive and Negative Affect Schedule (PANAS). The PANAS (Watson, Clark, \& Tellegen, 1988; Dutch translation by Peeters, Ponds, \& Vermeeren, 1996) is a scale designed to measure the experience of several positive and negative affects during the past two weeks, such as: 'interested', 'determined', 'guilty' or 'distressed'. Ratings are made on a 5-point Likert-scale, ranging from 'very slight or not at all' to 'extremely'. Higher scores indicate more positive and/or negative affect. The PANAS has high reliability and internal consistency (Crawford \& Henry, 2004; Watson et al., 1988).

Beck Depression Inventory-II (BDI-II). The BDI-II (Beck, Steer, \& Brown, 1996; Dutch translation by Van der Does, 2002) is a self-report questionnaire consisting of 21 items. All items have four statements each, and the participants have to select the answer that best describes their feelings and behaviours in the last two weeks. For example, with regard to crying, the four statements are as follows: 'I don't cry more than I used to,' 'I cry more than I used to,' I cry about every little thing', and 'I would like to cry, but I can't'. Total scores range from o to 
63, with scores between o and 13 indicating no depression, scores between 14 and 19 mild depression, between 20 and 28 moderate depression, and higher than 28 severe depression. Whisman, Perez, and Ramel (2000) found good internal consistency and reliability for the BDI-II.

Dutch Eating Behaviour Questionnaire (DEBQ). The DEBQ (Van Strien, Frijters, Bergers, \& Defares, 1986; Van Strien, 2005) is a 33-item questionnaire designed to measure self-reported emotional eating, external eating and dietary restraint. All items are scored on a 5-point Likert scale ranging from 'Never' to 'Very often'. Examples of items are 'Do you have a desire to eat when you are feeling lonely?' (Emotional eating), 'If food smells and looks good, do you eat more than usual?' (External eating), and 'How often do you try not to eat in between meals because you are watching your weight?' (Dietary restraint). Means are calculated for the three subscales and higher scores (within a range from 1 to 5) indicate increased emotional, external or restrained eating.

Mood induction. Positive and negative mood were induced by means of movie excerpts. The use of film clips has proven to be a successful method in changing subjective states (Chua, Touyz, \& Hill, 2004; Cools, Schotte, \& McNally, 1992; Evers, et al., 2009; Sheppard-Sawyer, McNally, \& Fischer, 2000; Turner et al., 2010; Yeomans \& Coughlan, 2009). In the current study, the positive movie fragment consisted of two combined scenes. The first scene was taken from the tv-series 'Mr. Bean' (1990), and shows Mr. Bean struggling to copy answers from his neighbour while taking an exam. The second scene was taken from the movie 'When Harry met Sally' (1989), and depicts a male and female friend enjoying dinner, when at one point the female simulates an orgasm in front of the full restaurant. The negative fragment came from the movie 'The Green Mile' (1999), showing an innocent man undergoing his death sentence. For the neutral control condition, part of a documentary on fishing was shown. Participants were randomly assigned to one of the three conditions.

Manipulation check. Subjective mood states were measured using $100 \mathrm{~mm}$ Visual Analogue Scales (VAS). Seven VASs were administered at five time points during the experiment. The scales asked for how frightened, sad, happy, guilty, enthusiastic, nervous and ashamed one felt at the moment, and ranged from 'not at all' (0) to 'very much' (100). Higher scores indicated a higher experience of that particular emotion. 
Food intake. An alleged taste test was set up to measure caloric food intake in participants. The foods presented were salty crisps (5.45 kcal/gram), ketchup crisps (5.40 kcal/gram) and mixed white, milk and dark chocolate (5.25 kcal/gram), all in large glass bowls. To cater for differences in food preferences and to ensure food variety, our participants were presented with both sweet and savoury high-fat food items. Studies have shown that increased food variety diminishes sensory specific satiety (Brondel et al., 2009; Norton, Anderson, \& Hetherington, 2006). Bowls contained an average of 190.64 grams (1000 kcal) of chocolate, 225.46 grams (1229 kcal) of salted crisps, and 225.44 grams (1217 kcal) of ketchup crisps. They were weighed in a separate room before and after tasting and amount of calories eaten were calculated. We were interested in total caloric intake and the means and standard deviations of kilocalories presented and consumed during the taste test are presented in Table 2.1. There were no differences between conditions in amount of kilocalories presented during the taste test, $F(2,83)=0.22, p=.81$. To ensure the cover story of a taste test was convincing, a 24-item questionnaire was constructed, with items pertaining to the taste, structure and appearance of the food. Participants were told that they had 15 minutes to complete this paper and pencil questionnaire, and that they could eat as much of the food as they liked.

Table 2.1 Means and standard deviations of food presentation and food consumption in kilocalories per condition

\begin{tabular}{lcccccc}
\hline & \multicolumn{2}{c}{ Negative condition (N=28) } & \multicolumn{2}{c}{ Neutral condition ( $N=30)$} & \multicolumn{2}{c}{ Positive condition (N=28) } \\
\cline { 2 - 7 } & $\mathrm{M}$ & $\mathrm{SD}$ & $\mathrm{M}$ & $\mathrm{SD}$ & $\mathrm{M}$ & $\mathrm{SD}$ \\
\hline Food presentation & 3447.94 & 10.87 & 3446.01 & 13.52 & 3447.00 & 8.40 \\
Food consumption & 191.58 & 120.53 & 172.22 & 125.94 & 202.31 & 157.97 \\
\hline
\end{tabular}

Awareness check. Participants were asked to describe what they thought the current study was about. The answer to this question gives an indication of whether participants might be aware of the true purpose of the research.

\section{Procedure}

Upon entering the lab, participants were seated behind a computer and were told they were about to take part in an experiment investigating the influence of movie clips on taste perception. The procedure started with filling out the first series of VAS mood scales, followed by watching either a positive, negative or neutral film fragment. Participants were instructed to give in to the feelings the movie fragment evoked in them. When the fragment was finished, participants filled out the VAS scales for the second time. The experimenter 
then left the room and came back with three bowls of food containing ketchup crisps, salty crisps and small chocolates for the taste test. A glass of water was also provided. In addition, they were provided with two more VAS scales assessing mood, and it was explained to them that they would hear a tone twice during the tasting (after 5 and 10 minutes), which indicated filling out a VAS scale. The experimenter then left the room to return 15 minutes later, administering the fifth and final mood measurement. After the taste test, participants filled out the BDI, PANAS, DEBQ and manipulation checklist, and height and weight were measured. To counteract possible long-lasting negative effects of watching the negative movie clip, participants in this condition were shown the positive fragment when they had completely finished the experiment.

\section{Data analysis}

One-way ANOVA's were used to check for pre-existing differences between conditions on age, BMI, depression, positive and negative affect, emotional and external eating, dietary restraint, and current mood. A 2 (time: before vs. after) × 3 (condition: negative vs. neutral vs. positive) MANOVA followed by simple effects analysis was performed to determine the success of the mood manipulation. For the main analysis, a hierarchical regression analysis was conducted. Gender, BMI, DEBQ external eating, DEBQ dietary restraint, and Negative Affect were taken into consideration as possible confounders. To avoid problems with multicollinearity, all variables were centred prior to entering the interaction term in the model. The variance inflation factor (VIF) and tolerance statistics indicated no evidence for multicollinearity, with VIFs close to 1 and tolerance never below 0.2. The Durbin-Watson statistic remained close to 2, thereby confirming that the assumption of independent errors was not violated. In addition, dummy variables were created for the three conditions (with the neutral condition acting as the reference group). To test the second hypothesis, bivariate and partial Pearson correlations were conducted. Three participants had incomplete (N $=1$ ) or outlying $(N=2)$ data regarding mood measurements, and were not included in the correlations.

\section{RESULTS}

\section{Participant characteristics}

A one-way ANOVA showed no pre-existing differences between participants in the positive (17 female, 11 male), negative (24 female, 4 male) and neutral conditions ( 23 female, 7 male), 
as shown in Table 2.2. A chi-square test indicated equal gender distribution over conditions, $X^{2}(2, N=86)=4.72, p=.094$.

\section{Mood manipulation}

Negative VAS ratings were reverse scored, added to the positive ratings and divided by two. This resulted in one single mood score for each time-point, with higher scores indicating a more positive mood. A 2 (time: before vs. after mood induction) $\times 3$ (condition: negative

Table 2.2 Mean and standard deviation of participant characteristics per condition

\begin{tabular}{|c|c|c|c|c|c|c|c|c|}
\hline & \multicolumn{2}{|c|}{ Negative condition $(\mathrm{N}=28)$} & \multicolumn{2}{|c|}{ Neutral condition $(\mathrm{N}=30)$} & \multicolumn{2}{|c|}{ Positive condition ( $\mathrm{N}=28)$} & \multirow[t]{2}{*}{$\mathrm{F}$} & \multirow[t]{2}{*}{$p$} \\
\hline & M & SD & M & SD & M & SD & & \\
\hline Age & 21.54 & 1.58 & 21.23 & 1.50 & 21.14 & 1.60 & .49 & .62 \\
\hline BMl & 23.89 & 4.97 & 23.66 & 3.77 & 23.10 & 3.08 & .28 & .75 \\
\hline $\mathrm{BDI}$ & 5.75 & 4.43 & 5.13 & 4.40 & 5.71 & 3.94 & .19 & .83 \\
\hline Positive affect ${ }^{1}$ & 32.04 & 5.88 & 30.93 & 6.23 & 32.14 & 5.39 & .38 & .68 \\
\hline Negative affect ${ }^{1}$ & 17.86 & 6.09 & 18.43 & 5.90 & 18.79 & 6.80 & .16 & .86 \\
\hline Emotional Eating² & 2.38 & 0.79 & 2.18 & 0.76 & 2.13 & 0.64 & .93 & .40 \\
\hline External Eating ${ }^{2}$ & 3.22 & 0.59 & 3.19 & 0.48 & 3.35 & 0.60 & .61 & .55 \\
\hline Dietary Restraint ${ }^{2}$ & 2.20 & 0.86 & 2.52 & 0.76 & 2.59 & 0.89 & 1.72 & .19 \\
\hline Current mood ${ }^{3}$ & 75.39 & 9.17 & 69.92 & 12.55 & 73.89 & 6.88 & 2.39 & .10 \\
\hline
\end{tabular}

${ }^{1}$ Positive and negative affect measured by PANAS

${ }^{2}$ Emotional Eating, External Eating, and Dietary Restraint measured by DEBQ

${ }^{3}$ Mood measured by $100 \mathrm{~mm}$ VAS scales at baseline, higher scores indicate better mood

vs. neutral vs. positive) MANOVA on subjective mood ratings before and after viewing the movie fragments was conducted, with the first factor within subjects and the second factor between subjects. The analysis revealed a significant multivariate interaction effect, $F(2,83)$ $=25.16, p<.001$. Subsequent simple main effects showed no significant differences in mood between conditions at baseline, $F(2,83)=2.39, p=.09$. Mood induction was successful in the positive condition, with participants becoming more positive (pre, $M=73.89, \mathrm{SD}=6.88$; post, $\mathrm{M}=79.50, \mathrm{SD}=10.84, F(1,83)=9.66, p=.003)$ after watching the movie clip. In the negative condition participants were significantly more negative after the manipulation (pre, $M=75.39, S D=9.17$; post, $M=63.24, S D=12.98, F(1,83)=45.26, p<.001$ ). In the neutral condition, participant's mood did not change (pre, $M=69.92$, SD = 12.55; post, $M=69.66$, $\mathrm{SD}=11.49, F(1,83)=.02, p=.89)$. It was concluded that the mood manipulations were successful. 


\section{Hypothesis 1: After both negative and positive emotions, emotional eaters will consume more calories than non-emotional eaters. In addition, their caloric intake will be higher in both emotional conditions compared to the control condition.}

Participants in the three conditions did not differ in caloric intake, $F(2,83)=0.37, p=.69$, as shown in Table 2.1. In order to test the hypothesis, a hierarchical multiple regression was performed. Gender, BMI, DEBQ External Eating scores, DEBQ Dietary Restraint scores, and negative affect were entered in the first block as control variables. In the second block, condition and emotional eating scores were entered, and finally the Condition X Emotional Eating interaction terms were added in the third block. The results of the regression analysis are shown in Table 2.3. With regard to the control variables, only the DEBQ external eating score was a significant predictor, $\beta=.25, t(85)=2.12, p=.04$. The variables added in block 2 (condition and emotional eating scores) did not change the model significantly, $\Delta R^{2}=.005$, $p=.93$, but the third block revealed a significant effect of the interaction between condition (neutral vs. positive) and emotional eating scores on food intake $\Delta R^{2}=.07, p=.04$.

Simple slopes and interactions with standardized regression coefficients for participants scoring high (+ 1 SD; emotional eaters) and low (-1 SD; non-emotional eaters) on emotional

Table 2.3 Results of Hierarchical Regression Analysis ( $N=86)$

\begin{tabular}{|c|c|c|c|c|c|c|}
\hline \multirow[b]{2}{*}{ Variables and steps ${ }^{\dagger}$} & \multicolumn{6}{|c|}{ Caloric intake } \\
\hline & \multicolumn{2}{|l|}{ Step 1} & \multicolumn{2}{|l|}{ Step 2} & \multicolumn{2}{|c|}{ Step 3} \\
\hline & B & SE & B & SE & B & SE \\
\hline Gender & 25.14 & 37.27 & 27.00 & 38.46 & 15.71 & 37.70 \\
\hline BMI & -6.05 & 3.58 & -5.91 & 3.66 & -4.29 & 3.63 \\
\hline External Eating & $59.82^{*}$ & 28.22 & 57.37 & 31.62 & 45.74 & 31.23 \\
\hline Dietary Restraint & -20.98 & 20.68 & -21.87 & 21.97 & -29.71 & 21.52 \\
\hline Negative Affect & -1.54 & 2.48 & -1.47 & 2.52 & -1.08 & 2.46 \\
\hline Emotional Eating & & & .076 & 22.58 & -55.61 & 32.39 \\
\hline Condition (Neutral vs. Negative) & & & 7.01 & 35.52 & 7.28 & 34.66 \\
\hline Condition (Neutral vs. Positive) & & & 22.58 & 34.95 & 34.69 & 34.23 \\
\hline Emotional Eating X Neutral-Negative & & & & & 67.10 & 44.54 \\
\hline Emotional Eating X Neutral-Positive & & & & & $132.03^{*}$ & 51.10 \\
\hline$R^{2}$ & & & & & & \\
\hline$\Delta R^{2}$ & & & & & & \\
\hline
\end{tabular}

† Note: $\mathrm{B}$ is the unstandardized regression coefficient; ${ }^{*} p<.05$. 
eating are plotted in Figure 2.1. When comparing the emotional and non-emotional eaters, there was a trend-significant difference in the positive condition, in which emotional eaters tended to eat more than non-emotional eaters, $\beta=.42, t(85)=1.87, p=.07$. In the neutral condition there was a trend-significant difference in the opposite direction, $\beta=-.30, t(85)$ $=1.72, p=.09$, whereas there were no differences between emotional and non-emotional eaters in the negative condition, $\beta=.06, t(85)=0.34, p=.74$ Looking within the group of emotional eaters, there was significantly higher food intake in the positive compared to the neutral condition, $\beta=-.46, t(85)=2.43, p=.02$, and no difference between the negative and neutral conditions, $\beta=-.20, t(85)=1.24, p=.22$ or negative and positive conditions, $\beta=-.26$, $t(85)=1.44, p=.16$. There were no differences between conditions in the non-emotional eaters, all ts $<-1.31$, all $p s>$.19. In sum, emotional eaters ate more in a positive than in a neutral mood whereas there were no differences for non-emotional eaters. Negative mood had no effect on food consumption in both types of eaters (emotional and non-emotional).

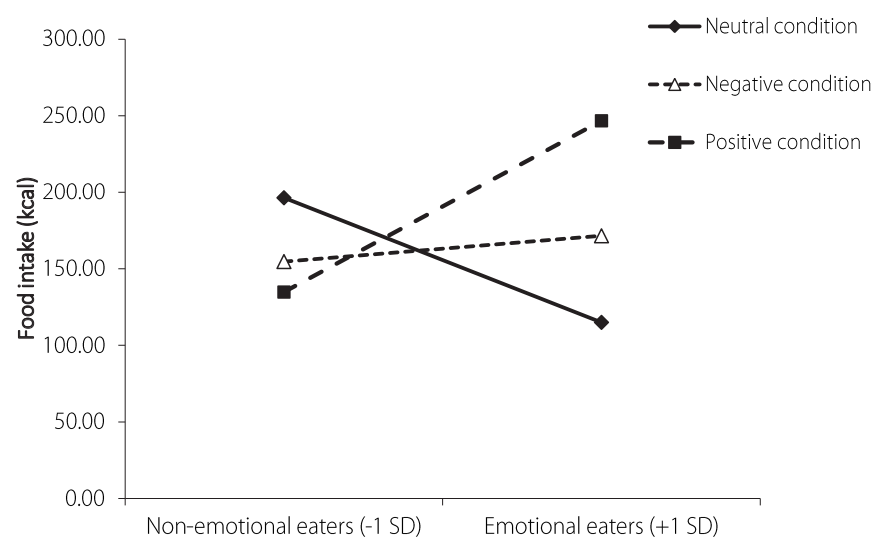

Figure 2.1 Food intake as a function of condition and emotional eating scores (-1 SD and +1 SD from the mean DEBQ score, respectively)

\section{Hypothesis 2: In self-reported emotional eaters, there will be a positive association between caloric intake and mood.}

Mood difference scores were calculated by subtracting mood immediately following viewing of the film clip from mood after 5, 10, and 15 minutes of tasting. Table 2.4 shows the mean mood scores on the four time points and before the film for the group as a whole, as well as separated for emotional and non-emotional eaters. A 2 (emotional vs. non-emotional eater) 
$X_{5}$ (mood at the 5 time-points) mixed ANOVA with Greenhouse-Geisser correction showed a main effect of mood, $F(2.30,191.21)=12.65, p=.00$. There was no significant interaction between mood and emotional eater type, $F(2.30,191.21)=1.84, p=.16$, nor was there was a significant effect of eater type, indicating that mood improvement for emotional and nonemotional eaters was the same, $F(1,83)=.24, p=.62$. Simple contrasts revealed that mood at each time-point during the taste test was significantly better than mood immediately after the film all Fs $(1,83)>16.51$, all ps < .001. In other words, mood increased significantly in the first five minutes of eating, and remained constant thereafter.

Due to the sample size and the fact that we do not expect differences between conditions in the mood effect of calorie intake, correlations over all conditions taken together are reported. Bivariate Pearson correlation coefficients for the group as a whole indicated an association between caloric intake and mood improvement after five minutes of eating. Follow-up partial Pearson correlations with DEBQ emotional eating scores as covariate led to similar results $(p=.052$ ). The results of the partial correlations indicate that emotional eating scores are not relevant to the association between caloric intake and mood improvement. All correlations are shown in Table 2.5.

Table 2.4 Means and standard deviations of mood scores before and during taste test

\begin{tabular}{|c|c|c|c|c|c|c|c|c|c|c|}
\hline & & & & & \multicolumn{6}{|c|}{ Minutes into taste test } \\
\hline & \multicolumn{2}{|c|}{ Before film } & \multicolumn{2}{|c|}{ After film } & \multicolumn{2}{|c|}{5} & \multicolumn{2}{|c|}{10} & \multicolumn{2}{|c|}{15} \\
\hline & $M$ & SD & $M$ & SD & $M$ & SD & $M$ & SD & $M$ & SD \\
\hline Total sample ( $\mathrm{N}=83$ ) & 72.84 & 10.13 & 71.17 & 12.58 & 75.51 & 10.50 & 74.80 & 11.00 & 75.35 & 11.55 \\
\hline Non-emotional eaters $(\mathrm{N}=46)$ & 72.50 & 10.28 & 72.54 & 12.12 & 76.00 & 10.87 & 75.12 & 11.08 & 75.71 & 11.53 \\
\hline Emotional eaters $(\mathrm{N}=37)$ & 73.26 & 10.07 & 69.47 & 13.09 & 74.91 & 10.14 & 74.40 & 11.03 & 74.90 & 11.71 \\
\hline
\end{tabular}

Table 2.5 Pearson correlations between calorie intake, emotional eating, and mood improvement

\begin{tabular}{lccc}
\hline & \multicolumn{3}{c}{ Minutes into taste test } \\
\hline & 5 & 10 & 15 \\
\cline { 2 - 4 } Bivariate correlations $(\mathrm{N}=83)$ & $.22^{* *}$ & .07 & .10 \\
Partial correlations $(\mathrm{N}=83)^{1}$ & $.22^{*}$ & .06 & .09 \\
\hline
\end{tabular}

** $p<.05,{ }^{*} p=.052$

${ }^{1}$ DEBQ emotional eating scores were added as a covariate in the partial correlations 


\section{DISCUSSION}

This study showed that self-reported emotional eaters respond in a different way to emotions than non-emotional eaters. Emotional eaters ate more in a positive mood compared to a neutral mood whereas non-emotional eaters ate about the same amount in both conditions. Within the positive mood condition, emotional eaters also tended to eat more than non-emotional eaters. Contrary to expectations, a negative mood did not increase the calorie consumption of emotional eaters. A significant positive correlation between calorie intake and mood improvement was found: the more calories participants ate in the first five minutes of tasting, the better their mood. However, being an emotional or non-emotional eater did not influence this association.

Our findings are in line with some previous studies, showing higher food intake after positive compared to negative emotions in non-depressed binge eaters (Dingemans, Martijn, Van Furth, \& Jansen, 2009), a non-clinical sample of students (Kenardy et al., 2003), and underweight people (Geliebter \& Aversa, 2003). Other researchers found that obese people (Patel \& Schlundt, 2001), restrained eaters (Cools et al., 1992), and people characterized by uncontrolled eating styles (Turner et al., 2010) consumed more in a positive than in a neutral mood. In short, these studies all point to increased food intake during positive emotions for eating- or weight concerned people (e.g. emotional and restrained eaters, as well as obese and underweight people). In a clinical or real-world setting, positive moods might be underestimated as a risk factor for overeating. However, mechanisms explaining why a positive mood would lead to overeating in these groups have not yet been identified.

Most self-reported emotional eating is related to negative moods and even the questionnaire we used to select emotional eaters, that is, the $D E B Q$, only asks for self-reported eating in response to negative moods. However, the high scorers on this questionnaire did not overeat in response to negative emotions whereas they did overeat in response to positive emotions. Why did emotional eaters not overeat in a negative mood?

One explanation is that the laboratory setting could have made participants feel observed and uncomfortable, which prevented them from overeating. Another possibility is that a standard mood manipulation like we used may not be able to trigger the right mood for overeating in every participant. A mood manipulation tailored to the individual might lead to more convincing results. However, naturalistic diary studies (Adriaanse et al., 2011; Conner, Fitter, \& Fletcher, 1999) were also unable to find effects of emotional eating scores on food intake after negative events. In addition, we did find overeating in the positive mood condition, in the same laboratory setting and also using a standardised mood induction. Kenardy 
et al. (2003) argued that emotional overeating may only be present in clinical samples. A further explanation could be that real emotional eaters do overeat in a negative mood, but that the questionnaire used in this study (DEBQ) was not sensitive or valid enough to truthfully measure emotional eating and identify emotional eaters. Indeed, some controversy has arisen on the validity of emotional eating scales. While it was empirically shown that emotional eating may occur, there is less consensus about emotional eating scales being the most fruitful way to identify emotional eaters. Evers et al. (2009) questioned the validity of emotional eating scales, stressing the difficulties and biases people encounter when recalling/judging their own behaviour. Agras and Telch (1998) suggested that a negative mood decreases the sense of control a binge eater has over eating, thereby estimating intake to be higher than it actually is. If this is applicable to self-reported emotional eaters or the population in general as well, people might easily overestimate their intake in negative moods and are therefore not able to fill out emotional eating questionnaires in an objective way. Adriaanse, de Ridder, and Evers (2011) proposed that it is the 'concerned eaters' (e.g., those who worry about and monitor their eating behaviour) who tend to score high on measures of emotional eating, while in fact not being emotional eaters at all. A similar suggestion of 'concerned eaters' was made by Jansen, Nederkoorn, et al. (2011), who found no discriminant validity between emotional, external and restraint eating scales.

The second aim of this study was to investigate the influence of food intake on mood. Emotional eating is supposed to exist because the act of consuming food is assumed to improve mood. We expected a mood improvement that is highly correlated with calorie intake in self-reported emotional eaters. Pearson correlations did confirm the hypothesis that mood improved by eating calories, but only in the first five minutes of the taste test, and for the whole sample, i.e. not only for emotional eaters. With regard to mood improvement after food consumption, our findings fit with the studies showing mood improvement after eating (Agras \& Telch, 1998; Dingemans, Martijn, Jansen, \& Van Furth, 2009a; Kenardy et al., 2003; Macht \& Mueller, 2007). In addition we showed that the amount of calories eaten is significantly correlated to the amount of mood improvement in the first five minutes of eating. Whereas Macht and Mueller (2007) reported stronger effects for emotional eaters, we did not find any differences between the two types of eaters: mood improved after eating, in both the emotional and non-emotional eaters.

One important limitation to mention with regard to the second hypothesis is that there was no non-eating control group. Therefore it is impossible to conclude that the changes in mood were actually due to food intake, and not to other factors, such as simply the passing of time. 
Another limitation of the study is that there were no hunger measurements taken. Hunger levels could influence food intake beyond the effects of mood, possibly confounding the results. However, since participants were randomised over conditions, this should not have caused differences between groups. Furthermore, one could argue that measuring eating behaviour in a lab environment is disadvantageous as well. Lab environments may prevent people (especially those who have issues concerning eating, such as emotional eaters) from eating as much as they would normally do. However, considering that increased food intake was found in emotional eaters in the positive condition, this concern is largely irrelevant. Finally, to avoid priming effects, the DEBQ was administered at the end of the experiment, after the mood manipulation and bogus taste test. However, we cannot exclude the possibility that the manipulation influenced scores on the questionnaire.

The present findings, although in need of replication, could be of value for the treatment of obesity. Considering that emotional eating is a risk factor for becoming obese (Hays \& Roberts, 2008), gaining a deeper understanding of the processes involved in emotional eating are of importance in combating the obesity epidemic. The current study underlines the importance of positive emotions in overeating, which are often overlooked.

\section{ACKNOWLEDGEMENTS}

This study is part of an ongoing project that is financed by the Netherlands Organisation for Scientific Research (NWO): Vici Grant 453.10.006, awarded to Anita Jansen. 

3

Happy eating: The Single Target Implicit Association Test predicts overeating after positive emotions

Published as:

Bongers, P., Jansen, A., Houben, K., \& Roefs, A. (2013). Happy eating: The Single Target Implicit Association Test predicts overeating after positive emotions. Eating Behaviors, 14, 348-355. 


\section{ABSTRACT}

For many years, questionnaires have been considered the standard when examining emotional eating behaviour. However, recently, some controversy has arisen about these questionnaires, and their usefulness in identifying emotional eaters has been questioned. The current study aimed to investigate the Single Target Implicit Association Test (ST-IAT) as a measure of emotional eating. Two ST-IATs (assessing food-positive and food-negative associations respectively) and the Dutch Eating Behaviour Questionnaire (DEBQ) were compared in undergraduate students. A positive, negative or neutral mood was induced by means of a film clip, and milkshake consumption was measured during and after the mood induction. It was hypothesized that participants with strong emotion-food associations on the ST-IATs (i.e., IAT-emotional eaters) would consume more food in the emotion induction condition corresponding to that emotion, as compared to those with weak emotion-food associations as well as to those in the neutral condition. Participants who scored high on both the positive and negative ST-IAT ate more during a positive mood induction than during a negative mood induction. This effect did not extend to milkshake consumption after the mood induction procedure. In addition, IAT-positive emotional eaters consumed more food than IAT-non-emotional eaters. No effects of the DEBQ on milkshake consumption were found. It is concluded that the ST-IAT has potential as a measure of emotional eating.

Keywords: Emotional eating; Implicit Association Test; Dutch Eating Behaviour Questionnaire; Food intake; Mood. 


\section{INTRODUCTION}

Most people have at one time or another experienced how emotional states influence their eating behaviour. They may have not been able to eat when grieving or in love, or celebrated happy events or achievements with big dinners. However, for some people, eating and emotions have become too interconnected. So much, in fact, that they have considerable difficulties distinguishing emotional arousal from feelings of hunger or food desires, and even confuse emotions for hunger or desire. This observation was first made by Bruch (1964), and in the years thereafter interest in the concept of 'emotional eating' has grown (Van Strien, Frijters, Bergers, \& Defares, 1986).

To measure emotional eating, several questionnaires have been developed (Emotional Eating Scale (EES) (Arnow, Kenardy, \& Agras, 1995); Emotional Overeating Questionnaire (EOS) (Masheb \& Grilo, 2006); Emotional Eating subscale of the Dutch Eating Behaviour Questionnaire (DEBQ) (Van Strien, Frijters, Bergers, \& Defares, 1986)). Although the design of these questionnaires may vary, they all have the same goal: to retrospectively self-assess eating behaviour in response to (mainly) negative emotions. A significant problem that arises with this kind of measures is that people are generally not very good at recalling their emotions, not good at recalling their eating behaviour, and not good at all at recalling associations between the two. Evers, de Ridder, and Adriaanse (2009) therefore suggest that emotional eating scales may suffer from a 'triple recall bias'. Along the same lines, the idea that emotions and eating are related is a kind of common sense in the general population, which may lead people to be biased towards experiences that confirm this notion (Ganley, 1989). These problems with current emotional eating scales might explain recent findings that show that scores on such scales are poor predictors of food intake following induction of a positive or negative mood. Evers et al. (2009) conducted five studies in which positive and/ or negative emotions were induced in emotional and non-emotional eaters as identified by the DEBQ subscale of emotional eating, before subjecting them to a bogus taste test. The way of inducing mood states (e.g., film excerpts or false feedback) and the type of mood induced (e.g., sad, happy or anxious) varied over studies. None of the studies showed increased food intake in DEBQ-emotional eaters as compared to non-emotional eaters, in either emotional or neutral conditions. Conner, Fitter, and Fletcher (1999) conducted a study in a more naturalistic setting in which participants kept track of daily hassles (i.e., stress) and filled out food diaries for one week. They found that, although the amount of daily hassles was positively related to snacking, this relationship was moderated by external eating (i.e. eating after being exposed to food stimuli), but not DEBQ-emotional eating. Another 
naturalistic study (Adriaanse, de Ridder, \& Evers, 2011) was also unable to discover an influence of DEBQ-emotional eating scores on snacking, neither under neutral nor emotional circumstances. Adriaanse et al. (2011) subsequently hypothesized that emotional eating scales measure a person's beliefs about the association between emotions and eating, but not their actual eating behaviour in response to negative emotions. These personal beliefs would follow from concern about one's own eating behaviour. Indeed, Adriaanse et al. (2011) showed that higher self-reported emotional eating scores are significant correlates of more worrying about and monitoring of one's own eating behaviour, lower control over one's eating behaviour, and a higher extrinsic motivation for healthy eating. A similar suggestion was made by Jansen, Nederkoorn, et al. (2011), who found that there was no discriminant validity of the emotional and external eating scales of the $\mathrm{DEBQ}$, and a moderate correlation between the subscales. Jansen, Nederkoorn, et al. (2011) concluded that the DEBQ, and possibly other eating scales as well, do not distinguish between emotional, external and restraint eaters, but simply indicate a general 'eating concern' in high-scoring people. A final problem with the emotional eating scales is that they mostly focus on negative emotions, while neglecting positive emotions, even though research has shown that positive mood can also increase food intake compared to neutral mood (Cools, Schotte, \& McNally, 1992; Patel \& Schlundt, 2001; Turner, Luszczynska, Warner, \& Schwarzer, 2010).

To overcome the problems related to self-report eating scales, the present study tested whether a Single Target Implicit Association Test (ST-IAT; Karpinski \& Steinman, 2006) is a better measure of emotional eating. The Implicit Association Test (IAT; Greenwald, McGhee, \& Schwartz, 1998) is an implicit measure of associations between concepts. Implicit measures are defined by De Houwer, Teige-Mociegemba, Spruyt, and Moors (2009) as "measurement outcomes that are causally produced by the to-be-measured attribute in the absence of certain goals, awareness, substantial cognitive resources, or substantial time" (p. 350). In the IAT, participants distinguish two categories of words (e.g., pleasant and unpleasant; the attributes) presented on the screen, as well as two other categories of words or pictures (e.g., flowers and insects; the target-concepts), by either a left-hand or right-hand response. The general idea is that when associated attributes and target-concepts require pressing the same response button (e.g., right-hand response for flowers and pleasant words), responses are faster than when unassociated attributes and target-concepts share the same response key (e.g., right-hand response for insects and pleasant words). This difference in response time is taken as an implicit measure of an association between the target and attribute categories. The ST-IAT is a modification of the original IAT in that there is only one target category, and it has been found to be equally reliable as the IAT (Karpinski \& Steinman, 
2006). For the current study, the target-concept is food, and the attribute categories are 'emotional' and 'neutral'. To measure associations between food and both positive and negative emotions, two ST-IATs are administered.

The hypothesis is that high emotional eaters can be distinguished from low emotional eaters based on their ST-IAT performance: Participants with stronger ST-IAT food-emotion associations (referred to as 'IAT-emotional eaters') will (1) consume more food after an emotion induction than after a control procedure, and (2) show higher food intake in the emotional conditions than participants with weaker ST-IAT food-emotion associations (referred to as 'IAT-non-emotional eaters'). Furthermore, this overeating is expected to be emotion-specific: those scoring high on IAT-positive emotional eating will overeat in the positive mood condition, while IAT-negative emotional eaters will overeat in the negative mood condition. No effect of emotion induction on food intake is expected for the IAT-nonemotional eaters.

\section{METHODS}

\section{Participants}

Participants were 122 female undergraduate students at Maastricht University. They were recruited through advertisements at the university, announcing a study on the relationship between empathy and perception. Participants were instructed not to consume any food in the two hours prior to the experiment. No exclusion criteria were applied. Participants took part for either course credit or a monetary reward of $€ 7.50$. The study's procedure was approved by the local ethical committee.

\section{Measures}

ST-IAT. Stimuli of the ST-IAT included neutral words (6), emotion words (6) and food words (6). Attribute category labels were presented in the top left ('neutral') and top right ('emotion') corners of the screen, and these remained on screen during the task. The target category label (i.e., 'food') was situated either below the 'neutral' label or below the 'emotion' label. Stimulus words and pictures were presented in the middle of the screen. Participants were instructed to sort the presented stimulus words by a button press (left or right) according to the category labels on screen. Stimuli remained on the screen until a response was given. After a practice-block of 24 trials with only neutral and emotional words, the combined ST-IAT blocks were presented, each consisting of 84 trials. Of these trials, 60 were attribute 
Table 3.1 Overview of neutral words, positive emotional words, negative emotional words and food pictures used in the ST-IATS

\begin{tabular}{llll}
\hline Neutral words & Positive emotional & Negative emotional & Food (pictures) \\
\hline Average & Happy & Sad & Potato crisps \\
Undecided & Joyful & Angry & Magnum ice cream \\
General & Satisfied & Gloomy & Chocolate \\
Normal & Cheerful & Lonely & Chocolate ice cream \\
Usual & Merry & Scared & French fries \\
Common & Relieved & Disappointed & Cupcake \\
\hline
\end{tabular}

(i.e., emotional and neutral words) trials and 24 were target (i.e., food pictures) trials. In one block, target food pictures were combined with emotional words, whereas food pictures were combined with neutral words in the other block. Order of these blocks was counterbalanced over participants, and participants could take a short break between blocks. A red cross appeared shortly (500 ms) on the screen when a mistake was made. Within the food-emotion block, participants made 48 responses on the right response key, and 36 responses on the left response key. In the neutral-emotion block this was reversed, with 36 right-key and 48 left-key responses being made. Each participant completed two ST-IATs: one with neutral words, positive emotions and high-caloric food pictures, and one with neutral words, negative emotions and high-caloric food pictures. The order of the two STIATs was counterbalanced over participants, and participants started with the same block in both ST-IATS (i.e., if a participant started with the food-emotional block in the positive IAT, she also started with the food-emotional block in the negative IAT). Because research has shown that emotional overeating mostly involves palatable, high-caloric food (Ganley, 1989), only these types of food items were used in the task. Table 3.1 gives an overview of the neutral, positive emotional and negative emotional words that were used, as well as the content of the food pictures.

Films. Participants watched either a positive, negative or neutral film fragment, lasting for approximately 2 minutes and 45 seconds. The positive fragment was taken from the comedy film "When Harry Met Sally", showing a woman faking an orgasm in a full restaurant, in front of her male friend. The negative fragment was part of "The Champ", a film drama in which a young boy watches his father die after a boxing match. The neutral excerpt was taken from a BBC documentary on tidal waves in Australia. All three films were pilot tested among female students $(N=11)$ and were shown to induce the desired emotional effect: when watching the positive film, paired-sample t-tests showed an increase in positive mood (pre, 
$M=57.05, S D=17.08$; post $, M=66.77, S D=21.44, t(10)=3.76, p=.004)$ and a trend-significant decrease in negative mood (pre, $M=14.68, S D=13.65$; post, $M=8.36, S D=9.46, t(10)=2.04$, $p=.07$ ). With regard to the neutral film, no change in positive (pre, $M=62.45, S D=15.21$; post, $\mathrm{M}=58.50, \mathrm{SD}=19.73, \mathrm{t}(10)=0.99, p=.34$ ) or negative (pre, $\mathrm{M}=12.18, \mathrm{SD}=13.02$; post, $M=13.32, S D=13.66, t(10)=0.41, p=.69)$ emotions was observed. The negative film clearly elicited a negative mood, with an increase in negative emotions (pre, $M=14.64, S D=10.24$; post, $M=29.36, \mathrm{SD}=19.19, t(10)=3.48, p=.006)$ and a decrease in positive emotions (pre, $M=60.50, S D=18.16 ;$ post, $M=46.05, S D=23.14, t(10)=4.41, p=.001)$. In addition, both the positive and negative film clip were proven to be effective mood inducers in a previous study (Macht \& Mueller, 2007). Several researchers have shown that film clips are effective in inducing positive and negative emotional states (Chua, Touyz, \& Hill, 2004; Cools, et al., 1992; Evers, et al., 2009; Sheppard-Sawyer, McNally, \& Fischer, 2000; Turner, et al., 2010; Yeomans \& Coughlan, 2009), and Gerrards-Hesse, Spies, and Hesse (1994) recommend to use either a film or story when the aim is to induce both happy and sad feelings in one study.

Milkshakes. Three vanilla milkshakes were freshly made for each participant right before she started the experiment. Milkshakes consisted of 125 grams vanilla ice cream, 100 millilitres full milk, 8 grams vanilla sugar, and 25 grams whipped cream. They were served in $300 c c$ milkshake cups, covered with a lid, and a straw was provided. The milkshakes weighed an average of 249.94 grams and the total amount of kilocalories in one milkshake was 449.75 kcal. Milkshake intake was assessed by weighing milkshakes before and after the taste test. Participants were unaware of these measurements.

Film and taste questionnaire. The questionnaire consisted of 11 questions regarding the milkshakes and the film fragment. The questions were set up to match the cover story of the relationship between empathy and taste perception, and also to give participants the chance to drink as much of the milkshake as they would like. Nine questions referred to the taste of the milkshakes (e.g., for each milkshake how much they liked it and whether they all had the exact same taste), and two questions referred to the film clip. Except for the questions regarding liking of the milkshakes, none were included in the analyses.

Mood. Mood was measured by means of four $100 \mathrm{~mm}$ VAS scales, with 'not at all' anchored on the left side of the scale and 'extremely' on the right side. The scales were labelled sad, gloomy, happy and cheerful. 
$D E B Q$. The DEBQ (Van Strien, Frijters, Bergers, \& Defares, 1986) consists of three subscales with a total of 33 items; an Emotional Eating subscale with 13 items, and External Eating and Restraint subscales with 10 items each. All items are scored on a 5-point Likert Scale, ranging from 'Never' to 'Very often'.

Restraint Scale. The Restraint Scale (Herman \& Polivy, 1980) consists of 11 items, measuring attitudes towards eating, weight change and dieting. All items (except for item 10 and 11, asking about highest and ideal weight) are scored on 4 or 5 point Likert Scales, ranging from 'Never' or 'Not at all' to 'Always' or 'Very much'. The Restraint Scale was included to control for dietary restraint in participants, as research has shown that restrained and unrestrained eaters often differ in their food intake in the lab (e.g. Chua et al., 2004; Cools et al., 1992; Sheppard-Sawyer et al., 2000; Yeomans \& Coughlan, 2009).

Manipulation Check and Suspicion Probe. On the manipulation check form, participants filled out three VAS scales concerning how well they were able to concentrate on the film, how well they could taste the milkshake during the film, and how well they could taste the milkshakes after the film. Additional questions referred to their own description of what they thought the experiment was about, what they had lastly eaten and at what time, and whether they were currently dieting or restricting their food intake.

\section{Procedure}

Upon arrival in the laboratory, the participant was explained that the study was about empathy and taste perception. She signed an informed consent form and filled out a baseline VAS for mood. Subsequently, she was seated behind a computer to perform the positive emotions and negative emotions ST-IATs. After completion of the ST-IATs, the participant filled out another mood VAS. In the meantime, the experimenter collected the first milkshake from the fridge in a kitchen next door. The experimenter placed the milkshake in front of the participant, and gave the following instruction: 'You're about to see a film fragment and you should really focus and concentrate on the film, and empathize with it. While you do this, you should taste from this milkshake. However, you should not let the milkshake divert you from the film, it is important that you keep concentrating on the fragment. You will receive some questions later.' After this, the experimenter asked the participant to put on headphones, and started the film. The experimenter left the room, and returned when the film was finished. The participant received another mood VAS to fill out, and the experimenter replaced the first milkshake with two new ones, labeled A and B. The participant 
was informed she now had five minutes to complete a questionnaire with questions about the milkshake she drank during the film, the two new milkshakes, and the film. She was told that she could drink as much as she liked from the milkshakes. This was also repeated at the end of the questionnaire, where it said: 'You are now finished with the questionnaire. Wait until the experimenter returns. Feel free to drink some more of the milkshakes; left-overs will be thrown away.' The experimenter left the room and returned after 5 minutes. The participant filled out a fourth mood VAS, followed by a manipulation check, the DEBQ and RS. Finally, height and weight were measured, and the participant received her reward for participation. The whole procedure lasted approximately 40 minutes.

\section{Statistical analyses}

One-way ANOVA's were conducted to discover any pre-existing differences between the three groups, and paired sample t-test to check whether the mood manipulation was successful. Both scores on the positive scales were taken together and divided by two to calculate one general indicator of positive mood. The same was done with the negative mood scales. To investigate the usefulness of the ST-IAT over questionnaires when measuring emotional eating, a moderated regression analysis was carried out. Variables included in the regression were centred prior to entering them in the model. As indicated by Variance Inflation Factors (always close to 1) and tolerance (never below 0.2), there was no multicollinearity between the variables in any of the conducted regressions. In addition, the Durbin-Watson statistic had a value close to 2 in all analysis, revealing that the assumption of independence of errors was met.

\section{RESULTS}

\section{Participant exclusion}

Six participants were excluded because of missing data, misunderstood instructions (i.e., assuming that consumption of the whole milkshake was required during the film), or outlying (i.e., z-score > 3.29) data on age, BMI, or milkshake consumption. Four additional participants were eliminated because they were aware of the true purpose of the study. In total, 112 out of 122 participants were included in the analyses. 


\section{ST-IAT scores}

ST-IAT effects were computed with the D600 scoring algorithm, as suggested by Greenwald, Nosek, and Banaji (2003). Higher scores on the ST-IAT D600 indicate a faster response to emotion-food pairs than neutral-food pairs, and thus reflect a stronger association between emotions and food. In addition to the positive and negative ST-IATs separately, a combined average ST-IAT score was calculated by adding the scores on the negative ST-IAT to the positive version, and dividing it by two.

\section{Mood manipulation}

The mood manipulation was, despite careful pilot testing of the film clips, only partly successful. A 2 (time: before vs. after mood induction) × 3 (condition: positive vs. neutral vs. negative) MANOVA on subjective mood ratings before and after viewing the film fragments was conducted, with the first factor within subjects and the second factor between subjects. The analysis revealed a significant multivariate interaction effect for time $X$ positive mood $F(2,108)=75.23, p<.001$ as well as for time $X$ negative mood, $F(2,108)=48.05, p<$ .001. Subsequent simple main effects showed no significant differences in mood between conditions at baseline for the positive, $F(2,108)=.08, p=.93$ nor negative, $F(2,08)=2.02, p$ $=.14$ emotions. Participants in the negative condition increased in negative emotions (pre, $M=12.78, S D=10.75 ;$ post $, M=29.85, S D=18.73, F(1,108)=95.66, p<.001)$, while positive emotions decreased (pre, $M=67.78, S D=13.39$; post, $M=51.94, S D=16.70$ ) from pre to post test, $F(1,108)=91.90, p<.001$. In the positive condition, the opposite effect was found, with an increase in positive emotions (pre, $M=68.55, S D=15.61$; post, $M=79.30, S D=13.62, F(1$, $108)=43.49, p<.001$ ) and a decrease in negative emotions (pre, $M=14.58, S D=11.89$; post, $M=10.59, S D=10.83)$ from pre to post test, $F(1,108)=5.36, p=.02)$. So both the negative and positive mood inductions were successful. However, the neutral film had the same effect as the positive film, increasing positive (pre, $M=67.29$, $S D=13.50$; post, $M=73.62$, $S D=14.61$, $F(1,108)=15.50, p=<.001$ ) and decreasing negative emotions (pre, $M=18.24, S D=12.96$; post, $M=14.74, \mathrm{SD}=13.72, F(1,108)=4.25, p=.04)$. Therefore, the participants in the positive and neutral conditions were combined and treated as one positive mood induction, and in further analyses compared to the negative condition.

\section{Participant characteristics}

A one-way ANOVA was used to compare the participants in both conditions on several baseline characteristics (i.e., age, BMI, mood at the start of the experiment and mood before watching the film, DEBQ emotional eating scores, DEBQ external eating scores, restraint, 
Table 3.2 Means and standard deviations of participant characteristics per condition

\begin{tabular}{|c|c|c|c|c|c|c|}
\hline & \multicolumn{2}{|c|}{ Positive condition ( $\mathrm{N}=76$ ) } & \multicolumn{2}{|c|}{ Negative condition ( $N=36$ ) } & \multirow[t]{2}{*}{ F } & \multirow[t]{2}{*}{$p$} \\
\hline & M & SD & M & SD & & \\
\hline Age & 20.34 & 2.24 & 19.83 & 1.83 & 1.41 & .24 \\
\hline BMI & 22.56 & 2.30 & 22.23 & 3.09 & 0.33 & .57 \\
\hline Positive mood $1^{1}$ & 67.72 & 13.35 & 70.17 & 13.75 & 0.80 & .37 \\
\hline Negative mood $1^{1}$ & 15.55 & 11.62 & 11.69 & 9.58 & 3.00 & .09 \\
\hline Positive mood $2^{2}$ & 67.49 & 13.35 & 70.17 & 13.75 & 0.01 & .92 \\
\hline Negative mood $2^{2}$ & 16.70 & 12.63 & 12.78 & 10.75 & 2.59 & .11 \\
\hline Emotional Eating ${ }^{3}$ & 2.65 & 0.70 & 2.56 & 0.67 & 0.44 & .51 \\
\hline External Eating ${ }^{3}$ & 3.32 & 0.53 & 3.26 & 0.51 & 0.34 & .56 \\
\hline Dietary Restraint ${ }^{4}$ & 12.53 & 5.05 & 11.17 & 5.70 & 1.63 & .21 \\
\hline Positive ST-IAT score ${ }^{5}$ & 0.21 & 0.31 & 0.32 & 0.34 & 3.05 & .08 \\
\hline Negative ST-IAT score ${ }^{5}$ & 0.21 & 0.34 & 0.21 & 0.27 & 0.01 & .93 \\
\hline Hours since last meal & 3.41 & 1.84 & 2.84 & 1.17 & 3.91 & .05 \\
\hline
\end{tabular}

${ }^{1}$ Mood measured at baseline by $100 \mathrm{~mm}$ VAS scales, higher scores indicate better mood

${ }^{2}$ Mood measured after ST-IATs but before film by $100 \mathrm{~mm}$ VAS scales, higher scores indicate better mood

${ }^{3}$ Emotional Eating and External Eating measured by DEBQ

${ }^{4}$ Restraint measured by Restraint Scale

${ }^{5}$ ST-IAT scores computed with the D600 scoring algorithm, higher scores indicate higher degree of emotional eating

positive and negative ST-IAT emotional eating scores, and hours since last food intake). As Levene's test showed that homogeneity of variances was violated for $\operatorname{BMl}(F(1,110)=6.30$, $p=.01)$ and hours since last food intake $(F(1,110)=6.02, p=.02)$, Welch $F$ was used for these variables. As can be seen from Table 3.2, there were some marginally significant differences for hours since last food intake, as more time had passed for the positive condition compared to the negative condition. In addition, participants in the positive condition had slightly higher scores on the negative emotions at baseline, whereas people in the negative condition scored marginally higher on the positive ST-IAT.

\section{Correlations}

Pearson correlations were calculated for the negative ST-IAT, positive ST-IAT, and DEBQ emotional eating scale. Both ST-IATs correlated significantly with each other, $r=.34, p<.001$, but no correlation was found between the DEBQ emotional eating scale and the negative ST-IAT, $r=-.01, p=.92$, nor the DEBQ emotional eating scale and the positive ST-IAT, $r=.00$, $p=.99$. 


\section{Moderated regression}

The amount of milkshakes consumed (in grams) per condition is shown in Table 3.3. Milkshake intakes during the film, $F(1,110)=7.65, p=.007$, after the film, $F(1,110)=6.57, p=.01$, and in total, Welch's $F(1,101.54)=14.68, p<.001$ were higher in the positive compared to the negative condition.

The first regression analysis focused on milkshake consumption after watching the film clips. Scores on dietary restraint (RS), external eating (DEBQ), hours since last meal, ratings of milkshake palatability and grams of milkshake consumed during the film were entered as covariates in the first block. Only palatability ratings and previous milkshake consumption were significant and used in the subsequent analysis. In the second block, either the positive ST-IAT, negative ST-IAT, combined ST-IAT, or DEBQ emotional eating score was added, together with condition. The interaction between ST-IAT or DEBQ and condition was entered in the third and final block. Results showed no effects of ST-IAT, DEBQ, or condition on food intake, nor were there any interaction effects (positive ST-IAT, all ts $<1.27$, all ps $>.21$; negative ST-IAT, all ts < 1.42, all ps > .16; combined ST-IAT, all ts < 1.33, all ps > .19; DEBQ, all ts $<1.13$, all ps $>$.21).

In the second regression analysis, milkshake consumption during the film clip was entered as the dependent variable. Because one participant did not drink anything from the milkshake during the film, 111 participants were included in this analysis. No other changes were made, except that there was only one covariate, namely palatability of the milkshake consumed during the film. Different patterns emerged for the two types of ST-IAT, the combined ST-IAT, and the DEBQ, which are described in more detail in the following paragraphs. The results of the regression are shown in Table 3.4. Simple slopes and interactions with standardized regression coefficients for participants scoring high (+ 1 SD) and low (-1 SD) on all measures of emotional eating separately are plotted in Figure 3.1.

Table 3.3 Means and standard deviations of grams of milkshake intake during the film, after the film, and in total per condition

\begin{tabular}{lcccc}
\hline & \multicolumn{2}{c}{ Positive condition (N=76) } & \multicolumn{2}{c}{ Negative condition (N=36) } \\
\cline { 2 - 5 } & M & SD & M & SD \\
\hline Milkshake consumption during film & 71.46 & 47.03 & 46.49 & 39.01 \\
Milkshake consumption after film & 113.25 & 67.25 & 81.24 & 47.77 \\
Total milkshake consumption & 184.71 & 97.66 & 126.70 & 61.12 \\
\hline
\end{tabular}




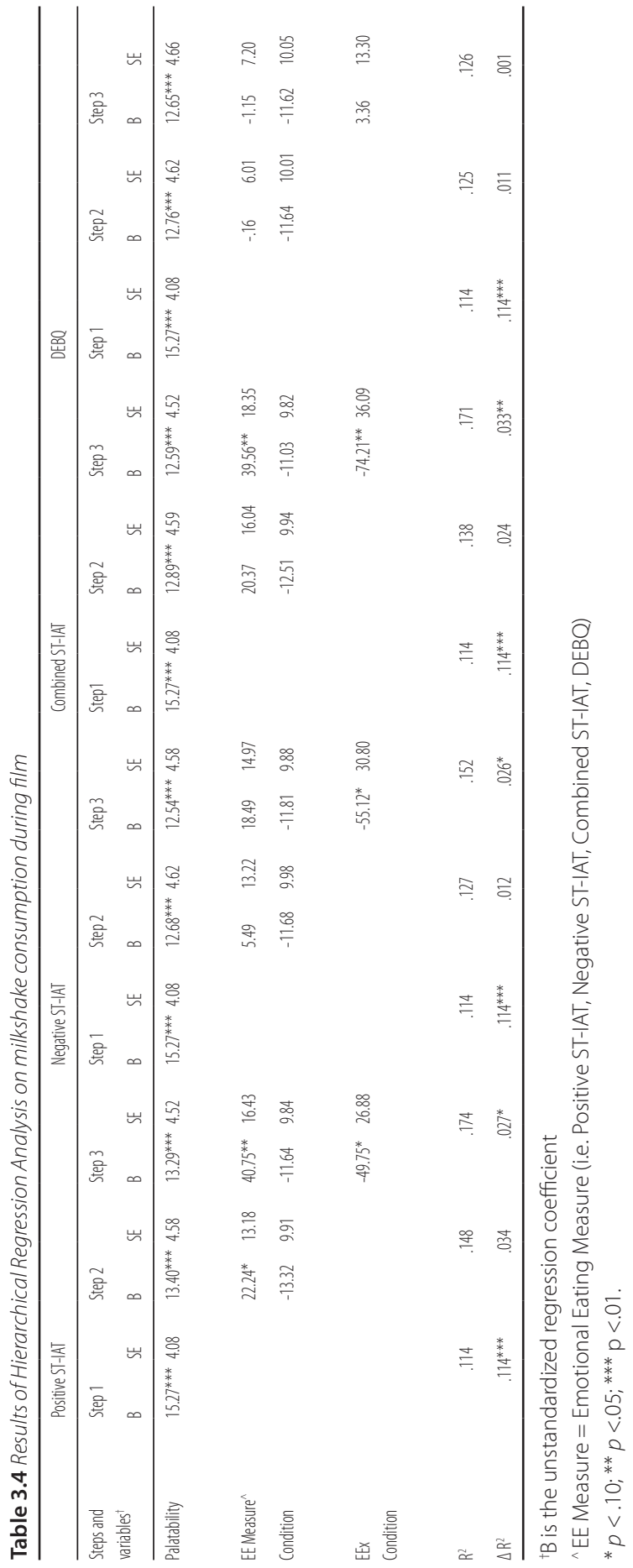



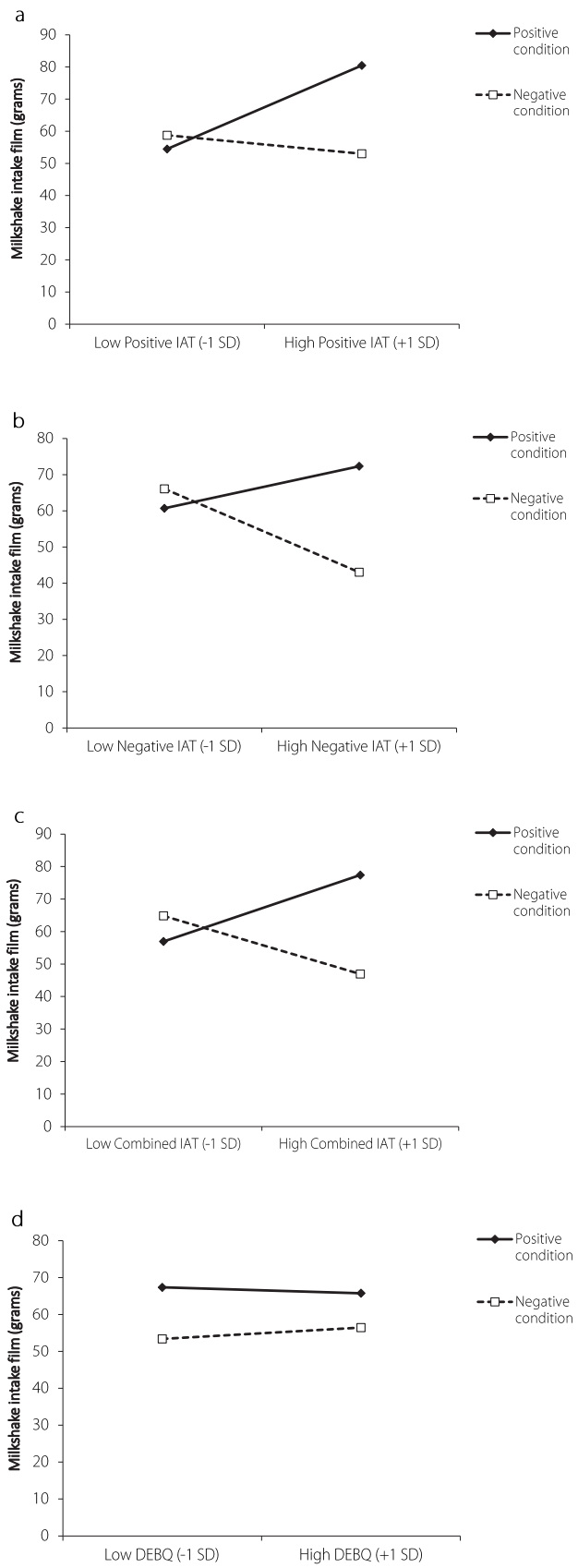

Figure 3.1 Milkshake consumption as a function of condition and low and high emotional eating scores $(-1$ SD and +1 SD from the mean) for the Positive ST-IAT (a), Negative ST-IAT (b), Combined ST-IAT (c), and DEBQ sCore (d). 


\section{Positive ST-IAT as measure of emotional eating}

Analyzing the positive ST-IAT as predictor of milkshake consumption during the film clip showed a trend-significant interaction between the positive ST-IAT and condition, $\beta=-.21$, $t(110)=1.85, p=.07$. More specifically, there was a significant difference between high and low positive ST-IAT scorers in the positive mood condition, with high scorers consuming significantly more milkshake than low scorers, $\beta=.28, t(110)=2.48, p=.02$, while there were no differences between high and low positive ST-IAT scorers in the negative mood condition, $\beta=-.06, t(110)=0.42, p=.67$. Looking at differences in food intake between the positive and negative conditions for low and high emotional eaters separately, there was no difference for low scorers, $\beta=.04, t(110)=0.31, p=.76$, but there was for high scorers. They consumed significantly more food in the positive compared to the negative mood condition, $\beta=-.28, t(110)=2.21, p=.03$. In sum, these findings confirm the hypothesis that IAT-positive emotional eaters consume more food than IAT-positive non-emotional eaters, and that this effect is specific to the positive mood condition.

\section{Negative ST-IAT as measure of emotional eating}

With regard to the negative ST-IAT, high and low scorers did not differ on food intake in either the positive, $\beta=.13, t(110)=1.24, p=.22$ or negative, $\beta=-.25, t(110)=1.36, p=.18$ mood condition. However, there was a trend-significant interaction between the negative ST-IAT and mood condition, $\beta=-.18, t(110)=1.79, p=.08$. The high ST-IAT emotional eaters consumed significantly more food in the positive than in the negative condition, $\beta=-.30$, $t(110)=2.10, p=.04$, while this was not found for those scoring low on negative ST-IAT emotional eating, $\beta=.06, t(110)=0.39, p=.70$. So, although IAT-negative emotional eaters did overeat after an emotion induction, they unexpectedly consumed more after positive than after negative emotions.

\section{Combined ST-IAT as measure of emotional eating}

When the positive and negative ST-IATs were combined into one single ST-IAT score, reflecting typical emotional eaters, a pattern similar to the one observed in the positive ST-IAT emerged, with a significant interaction between condition and ST-IAT, $\beta=-.21, t(110)=2.06$, $p=.04$. While there were no differences between high and low emotional eaters in the negative condition, $\beta=-.20, t(110)=1.12, p=.27$, there was again higher food intake in the positive condition for those with higher ST-IAT scores, $\beta=.22, t(110)=2.16, p=.03$ than those with lower scores. In addition, high scorers consumed significantly more milkshake when in a positive compared to a negative mood, $\beta=-.31, t(110)=2.32, p=.02$, whereas this did not 
occur within the group with low ST-IAT scores, $\beta=.08, t(110)=0.57, p=.57$. Thus, combined ST-IAT-emotional eaters consumed more milkshake than combined ST-IAT-non-emotional eaters, but only when in a positive mood.

\section{$D E B Q$ as measure of emotional eating}

The DEBQ revealed no significant effects. There was no interaction, $\beta=.03, t(110)=0.25, p$ $=.80$, nor were there any differences between the high and low emotional eaters in either mood condition (positive: $\beta=-.02, t(110)=0.16, p=.87$; negative: $\beta=-.04, t(110)=0.25, p$ $=.84)$. In addition, neither the low, $\beta=-.14, t(110)=1.03, p=.31$ nor high emotional eaters $\beta=-.10, t(110)=0.68, p=.50$ differed in food intake between conditions. Taken together, DEBQ-emotional eating scores did not predict food intake.

\section{Spin-off: Milkshake palatability}

Although not directly related to the hypotheses, but of relevance for the study, was an interesting finding with regard to milkshake palatability that emerged during analyses. Independent samples t-tests showed that participants in both mood conditions rated the two milkshakes tasted after the film equally palatable (first milkshake: positive condition, $M$ $=3.38, \mathrm{SD}=.96$, negative condition, $\mathrm{M}=3.10, \mathrm{SD}=.94, t(115)=1.51, p=.13$; second milkshake: $\mathrm{M}=3.46, \mathrm{SD}=.96$, negative condition, $\mathrm{M}=3.18$, $\mathrm{SD}=.91, t(115)=1.52, p=.13)$. However, the same milkshake consumed while watching the film was rated significantly tastier by the participants who had watched the positive film, $M=3.82$, SD $=.85$ compared to those who had watched the negative film, $M=2.74, \mathrm{SD}=.99, t(114)=6.06, p=.00$. In addition, paired samples t-tests revealed that while participants in the positive condition rated the milkshake consumed during the movie $(M=3.82, \mathrm{SD}=.85)$ as more palatable than the milkshakes after the movie (first milkshake: $M=3.38, \mathrm{SD}=.96, t(76)=4.33, p=.00$; second milkshake: $M=3.45, \mathrm{SD}=.97, t(76)=3.79, p=.00)$, this effect was opposite for the negative condition, where the after-movie milkshakes were judged to be tastier (first milkshake: $M$ $=3.10, \mathrm{SD}=.94, t(38)=2.02, p=.05 ;$ second milkshake: $\mathrm{M}=3.18, \mathrm{SD}=.91, t(38)=2.49, p=$ .02) compared to the milkshake ingested during the movie $(M=2.74$, SD $=.99)$. In sum, these results show that emotional stimuli presented during milkshake consumption may influence its palatability. 


\section{DISCUSSION}

The present study was designed to investigate the ST-IAT as a predictor of emotional eating, and to compare it to the prevailing method of emotional eating measures, namely questionnaires. In general, for both separate positive and negative ST-IATs and the combined score, differences emerged in milkshake consumption in emotional and non-emotional eaters while watching a movie, depending on the induced mood. More specifically, with regard to the positive ST-IAT, results showed that IAT-positive emotional eaters in the positive condition consumed significantly more milkshake than people without these associations, and they also drank more in the positive than in the negative condition. The negative ST-IAT revealed a significant difference for IAT-negative emotional eaters, who, contrary to our hypothesis, consumed more milkshake while watching a positive film compared to a negative film. When the two ST-IATs were combined into one, IAT-emotional eaters displayed higher milkshake consumption than IAT-non-emotional eaters in the positive condition, and the same difference between conditions emerged for the high scoring group: IAT-emotional eaters drinking more milkshake in a positive mood. Finally, looking at the $D E B Q$, there were no differences between the two types of eaters. Both DEBQ-emotional and DEBQ-nonemotional eaters consumed the same amount of food in each condition, and there were no differences between mood conditions within the groups.

In short, IAT-emotional eaters in the present study consume more food when in a positive mood compared to a negative mood. Moreover, in the case of the positive and combined ST-IAT, they consume more than IAT-non-emotional eaters when in a positive mood, and with all three ST-IAT measures food intake remains constant over groups when in a negative mood. There were no intake differences at all between DEBQ-emotional and DEBQ-nonemotional eaters. These findings only apply to food consumption during the experience of emotions; milkshake consumption after mood induction was not influenced by any of the emotional eating scores.

The findings on the DEBQ are not completely unexpected. Bongers, Jansen, Havermans, Roefs, and Nederkoorn (2013) also did not find DEBQ-emotional eaters to consume more food when in a negative mood as compared to DEBQ-non-emotional eaters, although they did find an increase in food consumption in the emotional eaters when they were in a positive state as compared to a neutral state. However, some other recent studies (Evers et al., 2009; Kenardy, Butler, Carter, \& Moor, 2003) found no effect of DEBQ-scores on food intake in emotional eaters, neither after a negative nor after a positive mood manipulation. Interesting to note is that while emotional eating has always been thought to happen in 
response to negative emotions (Schachter, Goldman, \& Gordon, 1968; Van Strien, Frijters, Bergers, \& Defares, 1986), these recent study results are consistent in showing that emotional eaters actually do not overeat in response to specifically negative emotions, neither when emotional eating is measured by self-report or by using an ST-IAT.

Increased food intake after positive or negative emotions is not something new; several researchers found this in for example the obese (Chua et al., 2004), restrained eaters (Cools et al., 1992; Sheppard-Sawyer et al., 2000; Yeomans \& Coughlan, 2009), and overweight people high in negative affect (Jansen et al., 2008). However, in emotional eaters it does not seem to be that straightforward. Why do emotional eaters not overeat after negative emotions? A first explanation is that they would overeat in a negative affective state in natural situations, but not in the laboratory. Participants might feel uncomfortable, self-aware and observed in a laboratory environment, which influences their food intake. However, because studies do show overeating after positive emotions, as well as overeating in other types of eaters, this does not seem a likely explanation. In addition, real-life diary studies conducted by Adriaanse et al. (2011) and Conner et al. (1999) also did not find effects of emotional eating scores on food consumption. A second possibility is that current measures of emotional eating are not valid, as was suggested by Adriaanse et al. (2011). Thirdly, it could be argued that negative emotional eating is only observable in a clinical population. Therefore, it would be interesting to repeat the current study in a clinical sample identified to suffer from emotional eating, such as binge eaters (Eldredge \& Agras, 1996; Pinaquy, Chabrol, Simon, Louvet, \& Barbe, 2003). Kenardy et al. (2003) suggested that only clinical samples increase food intake after negative emotions, or, alternatively, that a very strong mood manipulation is necessary to evoke these responses in non-clinical samples. In contrast to this, Macht and Simons (2000) found in their field study that students had a stronger motivation to eat after negative than after positive emotions. Fourth, overeating might only happen with certain negative emotions, and this might differ between people: Whereas some people overeat when feeling sad, others may only increase their food intake when angry or stressed. Current questionnaires, as well as the ST-IAT, do not differentiate between specific emotions. Perhaps there would be more convincing results with regard to negative emotions when they are tailored to the individual.

Another issue that warrants further explanation is that overeating only occurred during the emotional experience, but not after. Although these findings are in line with Evers et al. (2009) and Kenardy et al. (2003), who both found no differences between DEBQ-emotional and non-emotional eaters on food intake after a mood manipulation, they are in contrast with our (Bongers, Jansen, Havermans, et al., 2013) previous study, at least with regard to the 
positive emotions, during which DEBQ-emotional eaters overate. To our knowledge, the current study is the first that measured food consumption while undergoing an emotional experience, and it raises the question why overeating does occur at this point in time, but not afterwards. One possibility is that this is due to the food consumption during the film: there might have been differences in milkshake intake after the film if the participants had not already consumed some milkshake. A second possibility is that the effects of manipulations used in the laboratory fade away quickly, thereby being capable of eliciting increased food intake during the emotional experience, but not thereafter. Stronger manipulations, possibly tailored to the individual, might be more successful to induce longer-lasting effects. However, what these stronger manipulations should entail remains unclear; the aforementioned studies on emotional eating have used several methods of mood induction, such as film clips, bogus feedback on performance, vignettes describing emotional evens, and recalling sad memories, of which none appeared to be sufficient.

Deviating from the hypotheses of the study, but relevant to the obtained findings, is the surprising influence of emotional valence of movie fragments on taste perception. Participants in the negative condition rated the milkshake consumed during the movie as significantly less tasty than the milkshakes they received after the movie, even though all milkshakes were identical. It is interesting to see that judgments of taste depend on mood. This might mean that low mood makes food less tasty while being happy makes food taste better. Because palatability was added into the analyses as a covariate, this should not have influenced the results. The other way around, the film shown in the control condition elicited a positive mood in participants, which may have been caused by the simultaneous intake of a sweet, tasty, and high-caloric milkshake during this neutral manipulation. The control film clip was pilot tested before the study started, but without simultaneous milkshake intake, and was found not to interfere with the participant's mood. Although the influence of food on the appraisal of a neutral stimulus has never been tested directly, there is some indirect evidence that sweet and high-caloric foods can increase mood (Kenardy et al., 2003; Macht \& Dettmer, 2006; Macht \& Mueller, 2007). This might be a good example of evaluative conditioning during which a neutral stimulus changes in valence after being systematically paired with a liked or disliked stimulus: evaluative conditioning might explain why drinking a tasty milkshake during a neutral movie improves mood.

These results could also contribute to an explanation for the aforementioned issue of an absence of any effects in the negative mood condition. As the milkshakes appeared to have a positive effect on mood change after the neutral film, they might have been able to influence mood after the negative movie clip in a similar manner. The positive feelings evoked 
by milkshake consumption may have attenuated the negative feelings experienced during the negative manipulation. Therefore, although participants did decrease significantly in mood, this decrease may not have been enough to actually instigate overeating. When we apply this line of reasoning to the positive condition, it could be argued that the milkshake consumption intensified the positive emotions raised by the positive movie, therefore making it possible to find effects on food intake in this condition. Taking it one step further, these findings could explain why we did not find any effects on food intake after the film, or why some previous studies (e.g. Evers et al., 2009) failed to find a relationship between mood change and food consumption in emotional eaters: the manipulation was only strong enough when there was a combination of film and palatable food, and effects diminished when the emotion inducers are taken away.

Although the obtained findings are novel and cannot be generalized to other populations or contexts outside of the laboratory, the present results suggest that this ST-IAT has potential as a measure of emotional eating. Especially the positive ST-IAT showed predictive validity in distinguishing those who will or will not overeat after positive emotions. The negative ST-IAT was also able to differentiate between emotional and non-emotional eaters, although it unexpectedly predicted overeating after positive, and not negative, emotions. Future studies on its validity and usability in eating behaviour studies are needed.

\section{ACKNOWLEDGEMENTS}

This study is part of an ongoing project that is financed by the Netherlands Organisation for Scientific Research (NWO): Vici Grant 453.10.006, awarded to Anita Jansen. 




\section{4}

\section{'Emotional' does not even start to cover it:}

Generalization of overeating in emotional eaters

Bongers, P., de Graaff, A., \& Jansen, A. (in press). 'Emotional' does not even start to cover it: Generalization of overeating in emotional eaters. Appetite. 


\section{ABSTRACT}

Based on recent studies indicating that emotional eating is not the clearly defined problem it is often thought to be, the present study investigated whether emotional eaters overeat merely in response to negative emotional cues, or to other cues as well. It was hypothesized that emotional eaters would overeat after a variety of food cues, not limited to negative emotions. Participants took part in four conditions (negative mood manipulation, positive mood manipulation, food exposure and a control condition) divided over two sessions. Each condition was followed by a bogus taste test, after which food intake was measured. Results showed strong correlations between food intake after all four conditions, indicating that increased intake after one type of cue is related to increased intake after other cues. Participants were identified as emotional or non-emotional eaters based on food intake in the negative mood condition, and based on self-reported emotional eating scores. Both measures of emotional eating were significantly related to food intake after all cues. Based on the current findings, we conclude that individuals who show increased food intake when in a negative emotional state also overeat when experiencing other food-signalling cues. This indicates that 'emotional eating' may not fully capture the eating behaviour of individuals currently identified as 'emotional eaters'.

Keywords: Emotional eating; External eating; Food cue exposure; Cue-reactive eaters; Cue reactivity; Food intake; Types of eaters. 


\section{INTRODUCTION}

In eating research, it is common practice to use labels to define certain types of eaters. In the 1970s, Herman and Mack (1975) introduced the 'restrained eater', a term that is used to describe individuals who deliberately try to restrict their food intake to maintain or achieve their desired weight. Restrained eaters were later contrasted with disinhibited eaters (Stunkard \& Messick, 1985), to discriminate between those who are constantly able to restrict food intake, and those who tend to overeat or break their diets on a regular basis (Herman \& Polivy, 1975). Such disinhibiting factors leading to overeating could be internal cues (e.g., emotions), or external cues (e.g., the sight or smell of food), and two types of eaters have been presented accordingly: emotional eaters (assumed to be specifically responsive to negative emotions) and external eaters (assumed to be specifically responsive to external food cues) (Van Strien, Frijters, Bergers, \& Defares, 1986). These eater types are distinguished from restrained eaters, who are supposed to succeed in restraining their food intake (Van Strien et al., 1986). Currently, the distinction between emotional, external and restrained eaters is generally accepted, and the past 20 years have seen a wealth of studies devoted to these specific subtypes. Some clear empirical predictions follow from the division into these three eating types: individuals scoring high on measures of emotional eating should increase their food intake in response to the experience of (negative) emotions, high scorers on external eating scales should consume more in response to external cues, and those scoring high on restraint - but low on emotional and external eating- should not overeat.

However, recently there have been indications that emotional and external eating are not the clearly demarcated issues of overeating in response to negative emotions or external cues they have long been thought to be, but rather small aspects of a more general issue revolving around problematic food intake. Van Strien and Ouwens (2003) found that emotional eating, but not external eating or dietary restraint, moderated the relationship between a preload and food intake. Jansen et al. (2011) assessed degree of emotional eating, external eating and restrained eating in a female student sample. Unexpectedly, external eating scores did not predict food intake after exposure to food, and very similar eating patterns among high scorers on all three types of eating were found. Based on their data, Jansen et al. (2011) argued that there may be no need to distinguish between different types of eaters, but that high scorers on such scales are 'generally eating-concerned', whereas low scorers are unconcerned. According to the researchers, the eating-concerned individuals are characterized by an ever-present concern about their food intake as well as problems with restricting their food intake when confronted with intake-inducing cues such as emo- 
tions and palatable food. Along similar lines, studies taking a diary-approach were unable to relate emotional eating scores to food intake after the experience of daily hassles (Adriaanse, de Ridder, \& Evers, 2011; Conner, Fitter, \& Fletcher, 1999). However, they did identify snacking out of habit, restraint, and external eating as predictors of overeating after experiencing negative emotions. In an additional study, Adriaanse et al. (2011; study 3) found that high scores on emotional eating were predictive of eating concerns, specifically high worrying about and high monitoring of their own eating behaviour, low perceived control over the own eating behaviour, and stronger extrinsic motivation with regard to healthy eating. They proposed that people who score high on emotional eating are preoccupied with food and eating in general, and focus specifically on the negative aspects of eating.

Considering the aforementioned studies, it is conceivable that there is a bigger issue of general food responsiveness at hand and that in certain individuals many different cues will lead to overeating. This idea is further supported by studies showing strong associations between self-reported emotional eating, external eating, and dietary restraint (Jansen et al., 2011; Turner, Luszczynska, Warner, \& Schwarzer, 2010; Van Strien et al., 1986). In addition, there is some evidence that positive emotions can also induce overeating (i.e., higher intake in an experimental compared to a control procedure) in people who score high on an emotional eating questionnaire (Bongers, Jansen, Havermans, Roefs, \& Nederkoorn, 2013a). Insight into the cues that lead to overeating and whether individuals who report or display excessive food intake do so in response to only one specific cue or several cues is important for more effective prevention, assessment, and treatment of overeating.

The aim of the current study was to investigate food intake of emotional eaters in response to a variety of potentially food-signalling cues. Because substantially more studies have focused on emotional compared to external eating and some previous studies have questioned the validity of emotional eating questionnaires and classifications (see for example Adriaanse et al., 2011; Bongers et al., 2013a; Evers, de Ridder, \& Adriaanse, 2009), we use emotional eating as the reference point in this study. In addition, because recent studies have shown that high scores on questionnaires assessing eating after negative emotions do not necessarily correspond with actual eating behaviour after negative emotions (Adriaanse et al., 2011; Bongers et al., 2013a; Bongers, Jansen, Houben, \& Roefs, 2013b; Brogan \& Hevey, 2013; Conner et al., 1999; Evers et al., 2009), we sought to add to self-report questionnaires by including actual food intake after experiencing negative emotions to identify emotional and non-emotional eaters.

It is hypothesized that participants identified as emotional eaters will consume more food in a negative emotional state, in a positive emotional state and after food cue expo- 
sure compared to a control condition. No intake differences between conditions in the non-emotional eaters are expected. In addition, it is hypothesized that emotional eaters will consume more food than non-emotional eaters after all experimental conditions, but not the control condition.

\section{METHODS}

\section{Participants}

Participants were 42 female undergraduate students of Maastricht University, ranging in age from 19 to 27 years old $(M=20.26, S D=1.82)$. They were recruited through advertisements distributed throughout the university and online. The advertisements called for female undergraduate students in the ages 18 to 30 to participate in a study allegedly on taste perception under different circumstances. Students suffering from food allergies were excluded from participating. The study was approved by the local ethics committee.

\section{Conditions and manipulations}

The study employed a within-subject design, with participants partaking in all five conditions. The conditions were divided over two sessions one week apart, with each session containing one control condition and one emotional condition. The emotional conditions were divided over the two sessions to avoid difficulties in switching from positive to negative moods or vice versa in a short time-frame. One control condition was implemented in each session to check for increased food intake during the second session, in light of the possibility that participants felt more comfortable to eat upon returning to the lab. The exposure condition always took place in the first session. Order of the emotion conditions and of the conditions within sessions was counterbalanced. The conditions and sessions are depicted in Table 4.1.

Negative mood. While listening to personal sad music (see procedure), participants wrote down a sad memory. If they were to finish writing before the music ended, they were instructed to keep thinking about the sad memory. The manipulation lasted for 5 minutes, and was proven to be effective in earlier studies (Bongers, Van den Akker, Havermans, \& Jansen, submitted; Vuoskoski \& Eerola, 2012). 
Table 4.1 Overview of conditions per session

\begin{tabular}{ll}
\hline Conditions in Session 1 (week 1) & Conditions in Session 2 (week 2) ${ }^{1}$ \\
\hline Negative or Positive & Negative or Positive $^{2}$ \\
Exposure & Control \\
Control & \\
\hline
\end{tabular}

${ }^{1}$ Order of conditions was counterbalanced within sessions

${ }^{2}$ The emotional condition in session 2 was opposite from the emotional condition in session 1

Positive mood. This procedure was similar to the negative mood induction, except that participants listened to a personal happy piece of music, while thinking of and writing down a happy memory.

Food exposure. Participants were presented with two bowls containing two varieties of one of their top 3 chosen foods (e.g., for chocolate, they would receive M\&M's and Maltesers). For 3 minutes, they were instructed by the experimenter to smell the food and think about eating it, but not to actually eat it.

Control. In the control condition, participants solved connect-the-dots puzzles for 5 minutes. The puzzles ranged from 118 to 270 dots.

\section{Measurements}

Manipulation check. To evaluate successfulness of the manipulations, participants filled out four 10omm VAS scales before and after every manipulation. The VAS scales asked how sad, happy and hungry the participant felt, as well as how strong their desire to eat was. The scales ranged from 'not at all' to 'very much' for the measures of sadness, happiness and hunger, and from 'not strong at all' to 'very strong' for the desire-measure.

Food intake. Participants were presented with three types of food which they had selected as their favourites from five types of food before the start of the experiment. This selection was included to ensure food liking. For each type of food, two varieties were presented, as studies have shown that food variety counters sensory specific satiety (Brondel et al., 2009; Hetherington, Foster, Newman, Anderson, \& Norton, 2006). The types of food and their varieties (kcal per 100 grams reported in brackets) were: Chocolate - M\&M's (479 kcal) and Maltesers (498 kcal); Crisps - salty (555 kcal) and paprika (560 kcal); Peanuts - salted peanuts (615 kcal) and cocktail nuts (535 kcal); Cookies - mini chocolate chip cookies (505 kcal) and typical Dutch mini syrup waffles (445 kcal); Sweets - gummy bears (328 kcal) and 
gummy cola bottles (343 kcal). Food was presented in large bowls, containing between 553.97 grams (SD = 15.92; for crisps) and 1007.16 grams (SD = 96.10; for M\&Ms) of each food. For each participant, the top three foods were counterbalanced over conditions. The two control conditions and the two emotional conditions were paired with the same type of food (i.e., if a participant received chocolate during the first control condition, she received chocolate during the second control condition taste test as well). Participants filled out questions regarding the chosen foods during the bogus taste tests, which took place after every manipulation. Questions were asked about the palatability of the food, the flavour, and how the two food varieties compared to each other. Participants were instructed to taste of each food variety in order to answer the questions, and they were told that they were free to eat as much as they liked. Each taste test lasted for 5 minutes. Actual food intake was measured by weighing the bowls with food in a separate room before and after each taste test.

Dutch Eating Behaviour Questionnaire (DEBQ). The DEBQ (Van Strien, 2005) is a 33-item selfreport questionnaire measuring dietary restraint (DR; 10 items), external eating behaviour (EX; 10 items) and emotional eating behaviour (EE; 13 items). Questions are answered on a 5-point Likert Scale, ranging from 'never' to 'very often'. A mean score per subscale is calculated. Although the DEBQ has high internal consistency and factorial validity (Van Strien et al., 1986), the predictive and discriminant validity of the external (Jansen et al., 2011) and emotional subscales (Bongers et al., 2013a; Evers et al., 2009) is debatable.

Awareness check. A questionnaire was used to check whether participants were aware of the hypothesis of the study and whether they complied with the instruction to not eat in the 2 hours prior to the experiment.

BMI. BMI was obtained by measuring and weighing participants in the lab, while wearing street clothes and no shoes.

\section{Procedure}

Participants signed up for participation in a study on the palatability of food. They were instructed by email not to eat two hours prior to the experiment, and asked to rank five types of food (chocolate, crisps, peanuts, cookies, sweets) from most to least palatable. In addition, they were requested to fill out the DEBQ and to email back the completed questionnaire. Finally, they were asked to bring two songs that made them sad and two 
songs that made them happy with them to the lab on both testing days. Then dates for the first and second session were agreed upon. Upon entering the lab for the first session, the participant filled out an informed consent form and was informed about the procedure, using a cover story of taste perception under different circumstances. Then, the first mood VAS was filled out, followed by one of the manipulations (either negative or positive emotion, exposure, or control). The experimenter left the room during all manipulations, except for the exposure. After the manipulation, the participant was provided with another mood VAS. Subsequently, she was presented with two chosen bowls of food and filled out the taste questionnaire. The experimenter left the room during the 5 minutes of the taste test. Upon return, the experimenter took away the bowls of food and the participant relaxed for five minutes to make sure the effects of the manipulation and taste test would subside. Several magazines on gardening and home decoration were provided, carefully checked for the presence of eating-related advertisements or other food cues; whenever food was found in the magazines, the particular page was taken out. After relaxation, the exact same procedure was repeated for the other two manipulations. At the end, participants filled out a question regarding adherence to food intake restrictions, and the date for the second session was confirmed. The second session took place one week later, at the same time of day. The procedure was exactly the same as in the first session. The participant underwent the manipulation for the emotional condition opposite to the one in the previous session and a control condition. This order was counterbalanced across participants. At the end of the second session, the participant filled out the awareness check and height and weight were determined. Upon completion of the experiment, the participant was rewarded with course credits or a $€ 15$ voucher.

\section{Statistical analyses}

All intake data was converted from grams to kcal, and all analyses on intake use kcal consumed as the dependent variable. Intake in the two control conditions did not differ significantly (Control 1, $M=169.02, S D=105.81$; Control 2, $M=181.31, S D=119.31, t(41)=.76$, $p=.46)$, therefore one averaged variable of intake for the control condition was calculated and used in all analyses. Repeated Measures (M)ANOVAs with an adjusted alpha of .01 to correct for multiple testing were used to assess the effectiveness of the four manipulations (negative mood, positive mood, exposure and control). Pearson correlation coefficients were computed to assess associations between intake in different conditions. To analyze data with regard to the specific hypotheses, a Repeated Measures ANOVA with intake per condition (positive, exposure and control) as WS-factor and Z-transformed intake after 
negative emotions as covariate was conducted. A similar analysis was performed concerning Z-transformed self-reported emotional eating scores as covariate, with the addition of intake after negative emotions to the WS-factor. Greenhouse-Geisser corrections are reported whenever Mauchly's test indicated a violation of sphericity. Significant interactions were further investigated through spotlight analyses, in which intake was assessed at 1 SD below and 1 SD above the mean of emotional eating.

\section{RESULTS}

\section{General}

\section{Participant characteristics}

Participants' BMI ranged from 17.48 to $25.51(M=21.83, S D=2.14)$. DEBQ-EE scores ranged from 1.15 to $4.23(M=2.46, S D=.68)$. Compared to $D E B Q$-EE norm scores for female students (2.61-2.66; Van Strien, 2005), the mean score is slightly below average. The awareness check revealed that none of the participants was aware of the hypotheses of the study.

\section{Manipulations}

Four separate Repeated Measures (M)ANOVAs (WS-factor Condition: negative mood, positive mood, exposure and control) were conducted to assess changes in sadness, happiness, hunger and desire to eat in all five conditions. To correct for multiple testing across the five conditions, an alpha of .01 was applied to these analyses. The results are reported in Table 4.2. From the analyses it is clear that all manipulations were successful in reaching the intended effects (marked in grey). However, there was also a significant decrease in hunger and desire in the negative mood, and a small but significant increase in desire to eat during the second control condition. The effect of the negative mood manipulation on hunger and desire is not surprising as this is a normal response to aversive states, resulting from decreased gut activity (Wardle, 1990). 
Table 4.2 Mean and SD of VAS ratings before and after manipulation for each condition

\begin{tabular}{|c|c|c|c|c|c|c|c|c|}
\hline & & \multicolumn{2}{|c|}{ Before manipulation } & \multicolumn{5}{|c|}{ After manipulation } \\
\hline \multicolumn{2}{|l|}{ Condition } & M & SD & M & SD & $F$ & $p^{1}$ & $\eta^{2}$ \\
\hline \multirow[t]{4}{*}{ Negative mood } & $\mathrm{Sad}^{2}$ & 13.74 & 16.31 & 52.55 & 21.25 & 120.21 & .000 & .75 \\
\hline & Happy & 64.48 & 13.29 & 37.60 & 16.44 & 145.12 & .000 & .78 \\
\hline & Hungry & 55.62 & 19.01 & 44.50 & 19.11 & 26.76 & .000 & .39 \\
\hline & Desire & 60.45 & 17.76 & 44.60 & 22.12 & 29.38 & .000 & .42 \\
\hline \multirow[t]{4}{*}{ Positive mood } & Sad & 14.71 & 14.54 & 9.88 & 12.09 & 12.76 & .001 & .24 \\
\hline & Happy & 66.62 & 10.01 & 77.86 & 11.70 & 94.08 & .000 & .70 \\
\hline & Hungry & 52.14 & 20.47 & 53.07 & 20.59 & .50 & .48 & - \\
\hline & Desire & 56.83 & 17.35 & 57.24 & 18.54 & .05 & .83 & - \\
\hline \multirow[t]{4}{*}{ Food exposure } & Sad & 17.38 & 18.49 & 15.50 & 16.04 & 4.34 & .04 & - \\
\hline & Happy & 63.93 & 13.63 & 66.95 & 12.46 & 3.43 & .07 & - \\
\hline & Hungry & 49.10 & 23.66 & 54.21 & 22.52 & 11.17 & .002 & .21 \\
\hline & Desire & 52.69 & 20.75 & 64.48 & 19.92 & 25.62 & .000 & .38 \\
\hline \multirow[t]{4}{*}{ Control 1} & Sad & 16.86 & 16.74 & 15.21 & 15.92 & 2.77 & .10 & - \\
\hline & Happy & 65.74 & 11.36 & 68.95 & 10.98 & 5.84 & .02 & - \\
\hline & Hungry & 51.71 & 21.30 & 52.86 & 23.58 & .70 & .41 & - \\
\hline & Desire & 52.90 & 17.05 & 52.79 & 19.65 & .006 & .94 & - \\
\hline \multirow[t]{4}{*}{ Control 2} & Sad & 19.07 & 20.06 & 17.38 & 16.91 & 1.55 & .22 & - \\
\hline & Happy & 63.50 & 15.52 & 64.38 & 14.15 & .28 & .60 & - \\
\hline & Hungry & 55.98 & 18.31 & 55.74 & 19.78 & .04 & .84 & - \\
\hline & Desire & 56.93 & 20.50 & 60.14 & 20.43 & 7.59 & .009 & .15 \\
\hline
\end{tabular}

${ }^{1}$ An a of .01 was used to correct for multiple testing

${ }^{2}$ The highlighted data (grey) reflect the intended effects of the various manipulations

\section{Intake within sessions}

Participating in multiple taste tests within one session did not appear to affect food intake. Repeated Measures ANOVA showed that both within session 1 (test 1, $M=136.46, S D=89.70$; test $2, M=151.62, S D=80.41$; test $3, M=163.82, S D=97.12, F(2,82)=1.96, p=.15)$ and within session 2 (test 1, $M=165.91, S D=104.63$; test 2, $M=180.77$, $S D=110.04, F(1,41)=.76, p=.39$ ) the average amount of kcal consumed per taste test was equal. 


\section{Emotional eating - Actual consumption}

\section{Correlations}

Food intake in a negative mood correlated significantly with food intake in a positive mood $(r=.87, p<.001)$, food intake after food exposure $(r=.53, p<.001)$ and food intake after a control procedure ( $r=.48, p=.001)$. Thus, in line with our hypothesis, increased food intake after negative mood is strongly related to increases in food intake after a positive mood and intake after exposure. Unexpectedly, there was also a strong correlation between the negative mood and the control condition.

\section{Food intake}

The Repeated Measures ANOVA revealed a significant Condition X Emotional Eating interaction, $F(1.74,67.66)=3.88, p=.031, \eta^{2}=.08$, as well as a significant effect of Emotional Eating, $F(1,39)=54.63, p<.001, \eta^{2}=.58$. Pairwise comparisons with Bonferroni correction at 1 SD below (i.e., non-emotional eaters) and 1 SD above (i.e., emotional eaters) the mean of emotional eating showed no condition differences in non-emotional eaters (Positive Mood: $M=82.87, S E=9.64$; Exposure: $M=100.13, S E=14.46$; Control: $M=128.33$, $S E=20.12$; all $p$ 's > .14). In the emotional eaters, intake in both the positive $\operatorname{mood}(M=234.58, S E=9.44 ; p=1.0)$ and exposure $(M=179.66, S E=14.16 ; p=.096)$ conditions did not differ from intake in the control condition $(M=222.81, S E=19.71)$. There was however a significant intake difference

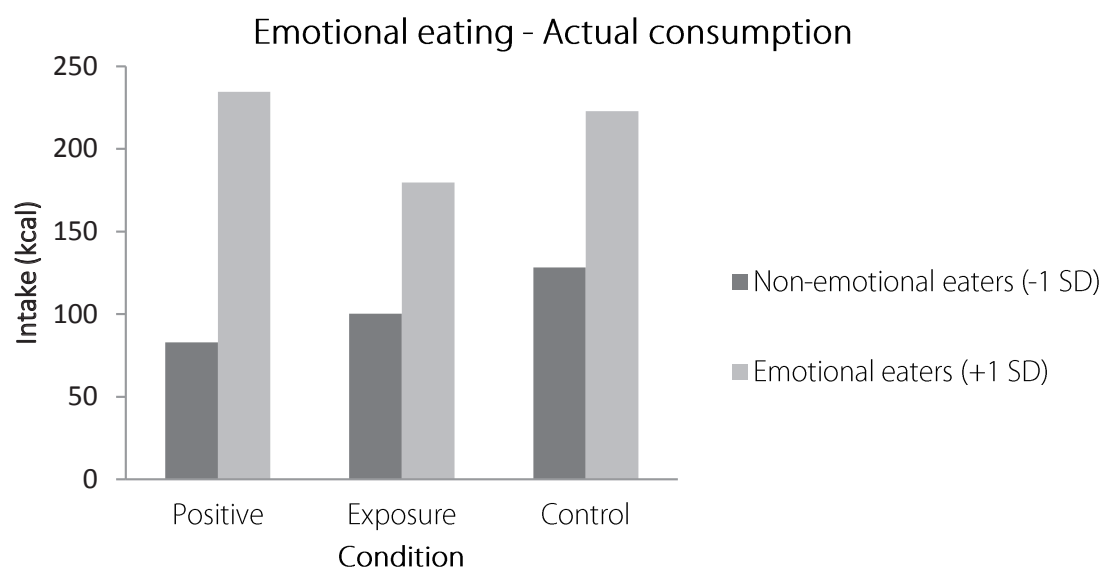

Figure 4.1 Caloric intake of emotional (1 SD above the mean) and non-emotional eaters (1 SD below the mean), based on actual consumption, in the positive mood, exposure and control conditions 
between the positive mood and exposure conditions, $p=.003$. Results are displayed in Figure 4.1. These findings indicate that emotional eaters (based on actual consumption) show overall increased food intake compared to non-emotional eaters, with intake differing across conditions only in the emotional eaters.

\section{Emotional eating - Self-report}

\section{Correlations}

There were small but non-significant correlations between the DEBQ-EE and the other DEBQ subscales (EE - EX, $r=.21, p=.18$; EE - RS, $r=.26, p=.10$ ). Self-reported emotional eating scores correlated significantly with intake in all conditions (negative mood, $r=.32$, $p<.042$; positive mood, $r=.32, p<.041$; exposure, $r=.31, p<.047$; control, $r=.31, p<.047$ ).

\section{Food intake}

The Repeated Measures ANOVA showed no significant Condition X Emotional Eating interaction, $F(2.14,83.62)=.08, p=.93$, nor a main effect of Condition, $F(2.14,83.62)=2.31, p=.10$. There was however a significant effect of Emotional Eating, $F(1,39)=6.30, p=.016, \eta^{2}=.16$. Results are plotted in Figure 2 . These data show that self-reported emotional eating scores are significantly related to increased food intake in all conditions, i.e. after a variety of cues.

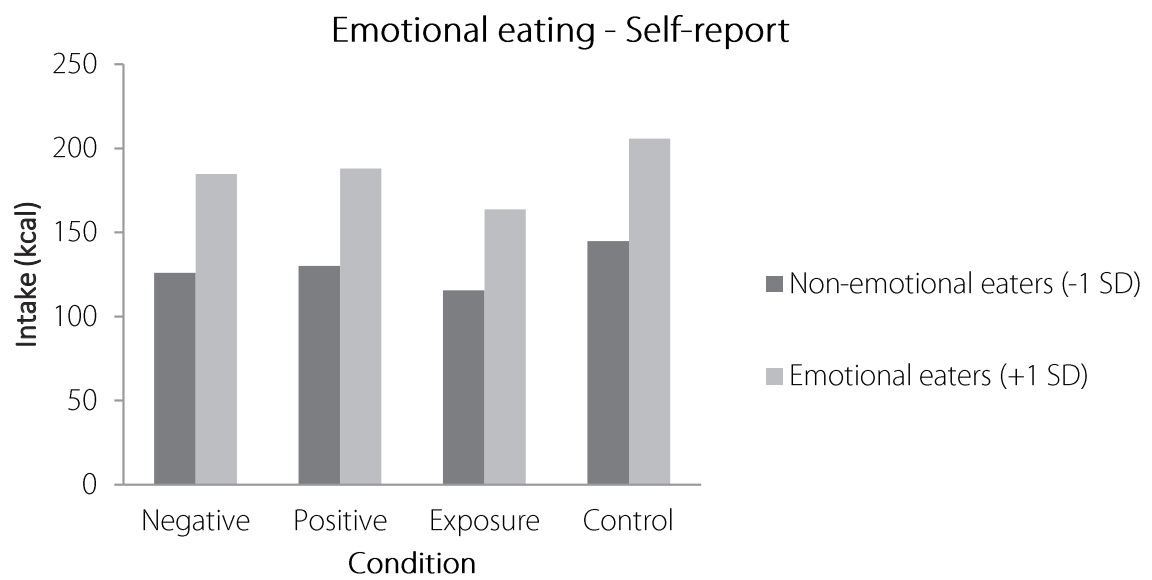

Figure 4.2 Caloric intake of self-reported emotional (1 SD above the mean) and non-emotional eaters (1 SD below the mean) in the negative mood, positive mood, exposure and control conditions 


\section{DISCUSSION}

In the current study we aimed to investigate whether people who overeat after experiencing negative emotions (based on both self-report and actual intake) are not merely emotional eaters, but instead overeat after a variety of food cues. The high correlations among intake during negative emotions, positive emotions, and after food exposure support this idea: increased intake after negative emotions is associated with increased intake in response to other cues, both in self-reported emotional eaters and emotional eaters identified by actual food intake. In addition to this, we also made predictions with regard to emotional versus non-emotional eaters. More specifically, we expected emotional eaters to show increased food intake in every experimental condition compared to the control condition, while we expected no differences in food intake in any of the conditions in the non-emotional eaters. Furthermore, we hypothesized that in all experimental conditions, but not the control condition, emotional eaters would consume more food than nonemotional eaters. The latter prediction was partly confirmed: emotional eaters tended to consume more food in all conditions, including the control condition. With regard to the first hypothesis, as predicted, the non-emotional eaters consumed equal amounts of food under all circumstances. However, the emotional eaters - at least when identified on basis of their intake - consumed more food in the positive mood than in the exposure condition, but neither condition differed from control. The data are in line with studies that show a strong correlation between questionnaire scores on emotional and external eating (Jansen et al., 2011; Turner et al., 2010; Van Strien et al., 1986) and studies that have shown increased food intake in response to positive emotions in emotional eaters (Bongers et al., 2013a). Furthermore, a recently published study (Vainik, Neseliler, Konstabel, Fellows, \& Dagher, 2015) showed that various eating related traits, including emotional eating, (i.e., emotional eating, attention paid to food, control over eating, eating impulsivity and binge eating) all share a similar underlying construct, which the researchers labelled 'uncontrolled eating'. With regard to intake in emotional eaters, the data show that self-reported emotional eaters consumed more food than non-emotional eaters in response to all cues. Emotional eaters classified on their actual intake also overeat in response to all cues, albeit to a lesser degree after exposure compared to when in a positive mood. It might be that food exposure is a different construct from emotions and does not lead to the same intake patterns. If so, however, it could be argued that the non-emotional control condition should also have led to different intake levels, and this was not observed. Together, the findings suggest that 
high levels of emotional eating are indicative of increased food consumption in general, and not specifically in response to negative emotions.

Interestingly, we also found high correlations between the experimental and control conditions and the control procedure seemed to elicit the exact same behaviour in participants as our experimental procedures did. One possibility is that certain individuals (i.e., those identified as emotional eaters) always eat more than other individuals (i.e., non-emotional eaters), no matter what the circumstances are. The mere presence of food during the taste test after the control condition was already enough to trigger increased intake. However, similar control conditions (i.e., taste test without a preceding manipulation) have been used numerous times without leading to an increase in food intake. On the other hand, it is also conceivable that the control condition might have unintentionally served as a fourth experimental condition: the knowledge that eating of high-caloric food would be necessary as a participant in the experiment, or having already consumed food in a condition preceding the control condition, could have served as triggers for eating. Similarly, it is possible that the puzzles we used caused boredom, ego depletion, stress, or feelings of disappointment or inadequacy, which could also all act to induce overeating (Greeno \& Wing, 1994; Groesz et al., 2012; Havermans, Vancleef, Kalamatianos, \& Nederkoorn, 2015; Kahan, Polivy, \& Herman, 2003; Sellahewa \& Mullan, 2015; Vohs \& Heatherton, 2000). Even though we instructed our participants that they could make the puzzles at their own leisure, and it did not matter how many they would finish, we cannot exclude the possibility that participants set selfimposed goals on how many of the puzzles they wanted to complete, and perhaps felt ego-depleted by the effort they put in, or disappointed when they did not reach this goal. It would be interesting to replicate the current study with a control condition that is unlikely to elicit feelings of boredom or a need to achieve. Future studies incorporating an improved control condition could elucidate whether the observed overeating in emotional eaters is conditional on the presence of food-related cues, or whether the mere availability of food is a cue in itself and sufficient to induce overeating.

It has repeatedly been shown that emotional eating does not predict food intake in response to negative emotions in both student (Adriaanse et al., 2011; Bongers et al., 2013a; Bongers et al., 2013b; Conner et al., 1999; Evers et al., 2009) and obese samples (Brogan \& Hevey, 2013). In contrast with these findings, but in line with some other studies (Raspopow, Abizaid, Matheson, \& Anisman, 2014; Van Strien et al., 2013; van Strien, Herman, Anschutz, Engels, \& de Weerth, 2012), the current results indicate that self-reported emotional scales may have at least some predictive validity, in the sense that individuals scoring high on this measure increased their food intake when in a negative mood. However, 'emotional 
eating' appears to be a misleading name that does not fully capture the eating behaviour of individuals currently named 'emotional eaters'. Indeed, emotional eaters overeat after a variety of cues, not restricted to negative emotions. If future studies replicate the current findings, 'cue-reactive eaters' might be a more appropriate name for these individuals.

The current study has some limitations that should be noted. First, the sample consisted of healthy young women, and therefore the results cannot be generalized to other populations, such as individuals who seek treatment, or those who are obese or otherwise eatingdisordered. The second limitation concerns the lab-setting the experiment was conducted in. It is possible that some individuals are more comfortable with eating in the lab than others, and therefore a lab-design might not accurately capture those specific individuals who overeat in response to negative emotions in real life. Third, although advertised as a study on taste perception under different circumstances, we cannot rule out the possibility that some participants were aware that we measured food intake and that this altered their behaviour. Fourth, questionnaires and behaviour can mutually influence each other. Although we aimed to minimize this effect by having participants fill out the DEBQ at the moment of study sing-up and not during one of the study sessions, we cannot exclude the possibility that filling out the questionnaire exerted some influence on eating behaviour. A final concern is the repeated taste tests in the study, both within and between sessions. It is possible that participating in taste tests in session 1 influenced participants' intake during the taste tests in session 2. In the current study this seems unlikely, given the finding that in the two control conditions in session 1 and session 2 intake was not significantly different. With regard to taste test influences within sessions, even little intake of food during one taste test might lessen hunger or could cause lesser intake in subsequent taste tests. However, because the order of manipulations was fully counterbalanced, if this effect was indeed present, it should have been the same for all conditions.

Taken together, the results of this study provide the first experimental evidence for the idea that so-called emotional eaters increase their food intake in response to a variety of cues. This raises the question whether'emotional eating' fully captures the eating behaviour of individuals classified as 'emotional eaters'. 


\section{ACKNOWLEDGEMENTS}

This study is part of an ongoing project that is financed by the Netherlands Organisation for Scientific Research (NWO): Vici Grant 453.10.006, awarded to Anita Jansen. We thank Angeliki-Maria Miliaraki for her help with data collection. 


5

Being impulsive and obese increases susceptibility to speeded detection of highcalorie foods

Published as:

Bongers, P., Van de Giessen, E., Roefs, A., Nederkoorn, C., Booij, J., Van den Brink, W., \& Jansen, A. (2015). Being impulsive and obese increases susceptibility to speeded detection of high-calorie foods. Health Psychology, 34, 677-685. 


\section{ABSTRACT}

Background: Overeating and obesity are associated with impulsivity. In studies among patients with a substance use disorder, impulsivity was found to be associated with substance-related attentional bias. Objective: To examine whether obesity, impulsivity and food craving are associated with a high calorie food attentional bias. Participants: Obese $(\mathrm{N}=185$, mean $\mathrm{BMI}=38.18 \pm 6.17)$ and matched healthy-weight $(\mathrm{N}=134$, mean $\mathrm{BMI}=$ $22.35 \pm 1.63)$ men (27.9\%) and women (72.1\%), aged 18-45 years. Measurements: Participants were tested on several self-report and behavioural measures of impulsivity (i.e., response inhibition and reward sensitivity) and self-reported trait craving. In addition, they performed a visual search task to measure attentional bias for high- and low-caloric foods. Results: Self-reported impulsivity influenced the relationship between weight status and detection speed of high- and low-caloric food items: High-impulsive participants with obesity were significantly faster than high-impulsive healthy-weight participants in detecting a highcaloric food item among neutral items, whereas no such difference was observed among low-impulsive participants. No significant effects were found on low-caloric food items, for trait craving or any of the behavioural measures of impulsivity. Conclusion: Self-reported impulsivity, but not trait craving or behavioural measures of impulsivity, is associated with an attentional bias for high-caloric foods, but only in people with obesity. It is in particular the speedy detection of high caloric-foods in the environment that characterizes the impulsive person with obesity, which in turn may cause risky eating patterns in a society were high-caloric food is overly present.

Keywords: Attention bias; Impulsivity; Food craving; Obesity; Toxic environment. 


\section{INTRODUCTION}

Attentional bias for high calorie food is putatively related to increased food cravings and a risk of overeating (Kemps \& Tiggemann, 2009; Smeets, Roefs, \& Jansen, 2009; Werthmann, Field, Roefs, Nederkoorn, \& Jansen, 2014; Werthmann et al., 2011). As such, this type of attentional bias can be viewed as an early cognitive part of a chain of subjective and behavioural cue reactivity responses (desire, craving, salivation, approaching) during food cue exposure. Cue reactivity, including attentional biases, to addiction-related cues has been associated with substance use and relapse risk (Field \& Cox, 2008) and recent studies have shown that the personality trait impulsivity is positively related to increased cue reactivity: more impulsive heavy drinkers show increased cue reactivity (craving) during cue exposure compared to less impulsive heavy drinkers (Papachristou, Nederkoorn, Havermans, Van der Horst, \& Jansen, 2012). Impulsivity is also purportedly positively related to attention biases for substance-related cues (Coskunpinar \& Cyders, 2013; Field \& Cox, 2008). Like substance use disorders, obesity and overeating are characterized by increased impulsivity. Higher levels of impulsivity are associated with (Galanti, Gluck, \& Geliebter, 2007; Guerrieri, Nederkoorn, \& Jansen, 2007) - and have even been found to lead to (Guerrieri, Nederkoorn, Schrooten, Martijn, \& Jansen, 2009; Guerrieri, Nederkoorn, Stankiewicz, et al., 2007; Jansen et al., 2009) - increased food intake.

Impulsivity is a multidimensional construct (Dawe \& Loxton, 2004; De Wit, 2009; Whiteside \& Lynam, 2001), which can be conceptualized in different ways. One such conceptualization is a division into three broad components: self-reported trait impulsivity, impaired response inhibition/impulsive action, and increased delay discounting/impulsive choice (Guerrieri, Nederkoorn, \& Jansen, 2008). All three impulsivity components have been linked to overeating and obesity (Guerrieri, et al., 2008; Mobbs, Crépin, Thiéry, Golay, \& Van der Linden, 2010; Nederkoorn, Smulders, Havermans, Roefs, \& Jansen, 2006; Rydén et al., 2003). This influence of impulsivity on overeating might moderate an attention bias for food. As impulsive people are characterized by, among others, increased distraction, acting without thinking, an inability to inhibit inappropriate responses and a hypersensitivity to immediate reward (Reynolds, Ortengren, Richards, \& De Wit, 2006), this could lead their attention to be more easily and more automatically drawn to salient and rewarding stimuli, such as high-calorie foods. In addition, they might also experience more difficulties in directing their attention away from such stimuli. Although several (Castellanos et al., 2009; Nijs, Muris, Euser, \& Franken, 2010; Werthmann et al., 2011), but not all (Nummenmaa, Hietanen, Calvo, \& Hyönä, 2011), studies have shown that attentional bias for high calorie food cues is more 
frequent in people with overweight and obesity than in healthy-weight participants, only one study investigated the relationship between impulsivity and food attentional bias (Hou et al., 2011). The authors found a positive correlation between self-reported trait impulsivity and attentional bias, as measured by a dot probe task. Other studies (Kemps \& Tiggemann, 2009; Smeets et al., 2009; Werthmann et al., 2011) found attention biases for food to be positively influenced by state craving, but no data are currently available on the association between attentional bias and a general trait craving for high calorie foods. Because different kinds of food constantly and simultaneously surround us, a general food craving in combination with an attention bias would make the current environment even more tempting and more difficult to resist.

Taken together, studies have clearly shown an association between impulsivity and increased food intake and overweight. In addition, in the field of substance use disorders higher levels of impulsivity are associated with biases in attention for substance cues. However, it is unknown whether impulsivity is also related to an attentional bias for tasty high calorie food cues, and if so, how weight status affects this relationship. In the present study, it is hypothesized that higher levels of impulsivity and trait food cravings are predictive of a stronger attentional bias, especially in participants with obesity.

\section{METHOD}

This research was part of a larger study on the heritability of obesity, which was conducted as collaboration between the Academic Medical Centre (Amsterdam, The Netherlands) and Maastricht University (Maastricht, The Netherlands). Participants were recruited from and tested either in the Amsterdam or the Maastricht area. The study procedures were approved by the Medical Ethics Committee of the Academic Medical Centre and the Psychology Ethics Committee of Maastricht University.

\section{Participants}

Participants ( $N=391)$ were recruited through advertisements in newspapers, supermarkets, hospitals, general practitioners, and obesity clinics. The advertisements called for Caucasian people, which was a requirement for the heritability aspect of the study, aged between 18 and 45 years, either healthy-weight (body mass index (BMI) 18-25 or obese (BMI >30), who were willing to participate in a study on the heritability of obesity. People with major neurological or psychiatric disorders, use of psychotropic medication, diseases affecting 
stomach, intestines, liver, kidneys or thyroid that could lead to an altered metabolism, and those who were pregnant or breastfeeding were excluded from participation. In addition, people who reported food deprivation were excluded. Participants with obesity and those of healthy weight were matched on a group level on age and gender. One participant was excluded because he reported not having had a proper meal in two weeks, and another participant for not following experimenter instructions. Twenty-five additional participants were excluded because their BMI was either below $18(n=2)$ or between 25 and $30(n=23)$. Finally, 45 healthy-weight participants did not match the obese group on the age-level. As age is a crucial factor, these participants were excluded. This left a sample of 319 participants: 134 healthy-weight (99 female, 35 male), and 185 with obesity (131 female, 54 male). Participants received a compensation of $€ 25$ and reimbursement for travelling expenses.

\section{Measurements}

Visual Search Task. The visual search task (based on Smeets et al., 2009; Smeets, Roefs, Van Furth, \& Jansen, 2008) is designed to measure attentional bias for food, and allows distinguishing between speeded detection of food and increased distraction by food. Diverging from the original task which used only chocolate and candy pictures, in the current task a distinction was made between high and low energy content to investigate whether attentional bias for food would be present for food in general, or for high-caloric food specifically. Food items that were used were typical high-caloric (e.g. ice cream, crisps, burgers, French fries, pizza) and low-caloric food (e.g. apples, crackers, carrots, grapes, broccoli). In addition, the original task used bags and couches as neutral stimuli, whereas bags and balls were used in the current task. Participants were presented with a screen showing a $5 \times 4$ matrix of pictures from two out of three different categories: neutral items, high caloric foods, and low caloric foods. The pictures were either all of the same category (odd-one-out absent), or one of them was from one of the other categories (odd-one-out present). The task for the participant was to decide as quickly and accurately as possible whether all pictures belonged to the same category, by pushing either a left ('all pictures belong to the same category') or right ('one picture belongs to a different category') button. Two different versions of the task were used in the study, counterbalanced over participants. In the first version, high-caloric food, low-caloric food, or a picture from neutral category I (e.g., balls) was presented among pictures of neutral category II (e.g., bags) for measuring detection, whereas a picture from neutral category II was presented among high-caloric food pictures, low-caloric food pictures, or pictures from neutral category I to measure distraction. In the second version, neutral category I was replaced by neutral category II, and vice versa. 
Before starting the task, participants performed 12 practice trials. The actual visual search task consisted of 154 trials (114 odd-one-out present and 40 odd-one-out absent), with a short break after 80 trials. During a trial, a short (100 ms) beep was presented, followed by a 500 ms fixation point. This was succeeded by the picture matrix, which remained on the screen until the participant responded or 20 seconds had passed. In case of a wrong, too slow, or too fast response, participants received feedback on the screen. A clear screen was presented for $1500 \mathrm{~ms}$ before the next trial started. Pictures were randomly allocated to their positions in the matrix, with the restriction that they were never placed immediately below or above the fixation point.

Scores for detection speed were calculated by subtracting response latencies on trials with a neutral picture from neutral category I (e.g., a ball) among neutral pictures from neutral category II (e.g., bags) from trials with a food picture among neutral pictures from category II (e.g., bags). Negative scores indicate faster detection of food items than of neutral items, while positive scores indicate the reverse (i.e., faster detection of neutral than of food items). Distraction was calculated by subtracting trials with one neutral picture from neutral category II (e.g., a bag) among neutral pictures from neutral category I (e.g., balls) from trials with one neutral picture from neutral category II (e.g., a ball) among food pictures. Positive scores are indicative of relatively more distraction by food than by neutral items, whereas negative scores indicate the reverse (i.e., more distraction by neutral than food items). Ultimately, each participant had a score for detection of high-caloric food, detection of low-caloric food, distraction by high-caloric food, and distraction by low-caloric food.

Stop Signal Task. The Stop Signal Task is a measure of impulsivity, based on the premise that impulsivity has to do with the inability to inhibit responses (Logan, Schachar, \& Tannock, 1997). Participants perform a reaction time task (go-task) in which they are required to respond to a stimulus as quickly as possible. Within a trial, a fixation cross (500 ms) is followed by either an arrow on the right of the computer screen pointing to the right, or an arrow on the left side pointing to the left. Correct responses are pressing a button on the right or left, respectively. However, in $25 \%$ of the trials, participants are presented with an auditory stop signal (i.e., a beep administered through headphones) immediately after the stimulus, indicating that they have to inhibit their response. The time between the stimulus and stop signal (stop delay) varies in steps of 50 ms depending on how well the participant is capable of inhibiting his response, ultimately resulting in an accuracy of around 50\% for everyone. By subtracting the mean stop delay from the mean reaction time on go-trials, the stop signal reaction time (SSRT) is calculated. A higher SSRT refers to less inhibitory control 
and higher impulsivity. In the current study, participants first performed 20 practice trials. These were followed by the actual task, consisting of 4 blocks containing 128 trials each. Participants could take short breaks in between blocks.

Delay Discounting. Delay discounting is a measure of impulsivity based on the premise that impulsivity has to do with an increased sensitivity for immediate reward. Participants are presented with choices between hypothetical monetary rewards, of which one is a smaller immediate reward and the other a delayed larger reward. The time delay was either 2 weeks, 1 month, 6 months, 1 year, 3 years, 5 years, or 10 years (presented in random order). The point at which someone has no preference for either the immediate smaller reward or the delayed larger reward at a specific time delay is the indifference point for that time delay. In the particular task used in this study, the immediate reward was successively approaching the indifference point depending on the person's choices and varied in value from $€ 12$ to $€$ 988. The delayed reward was fixed at $€ 1000$. The subject was presented 8 trials per time delay. Area under the curve (AUC) was calculated for all participants (Myerson, Green, \& Warusawitharana, 2001). AUCs were standardized to values between o and 1, with smaller AUCs reflecting steeper discounting and thus higher levels of impulsivity.

Barratt Impulsiveness Scale - version 11 (BIS-11). The BIS-11 (Patton, Stanford, \& Barratt, 1995) is a 30-item self-report questionnaire, designed to measure trait impulsivity. Items have to be answered on a 4-point scale, with higher total scores reflecting higher levels of trait impulsivity. The BIS-11 has high internal consistency, test-retest reliability, and convergent validity (Patton, et al., 1995; Stanford, et al., 2009). The total BIS-11 score was used in the analyses. In the current study, Cronbach's alpha for the BIS-11 was .78.

General Food Craving Questionnaire - Trait (GFCQ-T). The GFCQ-T is a self-report questionnaire focusing on a general trait for food craving and is a modification of the original version of the Trait Food Craving Questionnaire (TFCQ; Cepeda-Benito, Gleaves, Williams, \& Erath, 2000), translated and modified by Nijs, Franken, and Muris (2007), which measures craving for specific types of food. The GFCQ-T consists of 21 items to be scored on a six-point Likert-scale, ranging from 'never or not applicable' to 'always'. The questionnaire is reliable (as indicated by test-retest reliability and internal consistency) and valid with good discriminant validity and construct validity (Nijs et al., 2007). The total GFCQ-T score was used in the analyses. Cronbach's alpha in the current study was .95. 
External eating and dietary restraint. External eating and dietary restraint were measured by the External Eating and Dietary Restraint subscales of the Dutch Eating Behaviour Questionnaire (DEBQ; Van Strien, 2005; Van Strien, Frijters, Bergers, \& Defares, 1986). Both subscales consist of 10 items regarding eating in response to food cues in the environment (external eating) and restricting food intake (dietary restraint). Answers are given on a five-point Likert scale ranging from 'never' to 'very often'. Final scores range from 1 to 5 , with higher scores pointing to a higher degree of external eating or dietary behaviour. The scales have high internal consistency and factorial validity (Van Strien, Frijters, Bergers, \& Defares, 1986) and the Dietary Restraint subscale has moderate to good predictive validity (Van Strien, Frijters, Van Staveren, Defares, \& Deurenberg, 1986). However, a lack of discriminative and predictive validity for the External Eating subscale has been reported (Jansen, Nederkoorn, et al., 2011). Cronbach's alphas for the external eating and dietary restraint subscales in the current study were .84 and .89 , respectively.

Binge episodes. The Eating Disorder Examination (EDE; Cooper \& Fairburn, 1987) was used to assess the presence of binge episodes. The EDE is a semi-structured interview designed to measure eating psychopathology. For the present study, only questions on objective episodes of overeating were included. Participants were considered to have binge episodes when those occurred at least once in the past four weeks. The EDE has good discriminant validity (Cooper, Cooper, \& Fairburn, 1989) as well as moderate convergent validity (Rosen, Vara, Wendt, \& Leitenberg, 1990).

Demographic information. Demographic information was collected through questionnaires. Participants were asked about their age, gender, daytime activities (answering options: student, employed, unemployed, housewife/houseman, other), living situation (answering options: alone, together with partner, together with partner and children, alone with children, alone with roommates, with parents/caregivers/foster parents), highest level of completed education (answering options: no education, primary education, and all different levels of the Dutch educational system in secondary and further education), personal income and family income (answering options for both questions in Euro's: less than 1000 (i.e. \$1350), 1000-1500, 1500-2000, 2000-2500, 2500-3000, more than 3000).

Physical measurements. To calculate BMI, participant's height and weight were measured using a stadiometer (brand: Seca) and a digital weighing scale (brands: Seca and MyWeigh) while they were wearing street clothes and no shoes. Experimenters were trained in the 
use of the stadiometer and weighing scale, and all had prior experience in taking these measures. Participants were asked to remove heavy clothing, such as jackets or thick vests. Participants whose hairstyle impeded height measurement were asked to untie their hair. Measurements were done once, and BMI was calculated using the following formula: weight $(\mathrm{kg}) /\left(\right.$ height $\left.(\mathrm{m})^{2}\right)$.

\section{Procedure}

Participants were individually invited to the lab and were instructed to eat something small (e.g. an apple or sandwich) two hours before the start of the experiment to standardize hunger levels. Upon arrival they received an explanation about the study, and signed an informed consent form. They were then asked to take place behind a computer to execute the Delay Discounting Task, Stop Signal Task and the Visual Search Task. These computer tasks lasted for approximately 40 minutes in total. Instructions for the tasks were given by the experimenter and on the computer screen. At the end of the study, participants were interviewed about binge episodes, and filled out a battery of questionnaires, including demographic questions, the GFCQ-T, BIS-11, and DEBQ. Finally, height and weight were measured and the monetary compensation was given.

\section{Statistical analysis}

Outlying data (i.e. $z$-score > 3.29) on the Stop Signal Task $(n=2)$ and Visual Search Task (high-caloric detection, $\mathrm{n}=3$; low-caloric detection, $\mathrm{n}=1$; high-caloric distraction, $\mathrm{n}=1$; low-caloric distraction, $n=1$ ) were recoded into scores one unit (i.e., one ms) larger than the largest non-outlying score (Tabachnick, Fidell, \& Osterlind, 2001). With regard to the Visual Search Task, only odd-one-out present trials were included in the analyses. Trials with errors $(13.20 \%)$ or slow responding ( $M+3$ SD) to correct trials were discarded ( $0.27 \%$ of trials), as were trials slower than $20000 \mathrm{~ms}$ (did not occur in odd-one-out present trials) or faster than 200 ms (.013\% of trials). Due to technical problems, Stop Signal Task, Visual Search Task, and Delay Discounting data were missing for 34 (16 with obesity), 8 (2 with obesity), and 2 ( 1 with obesity) participants, respectively. One-way ANOVAs and Pearson chi-square tests were conducted to check for any pre-existing differences between the obese and healthy-weight groups. To test the hypothesis, moderated regression analyses with simple slope testing and spotlight-analyses at one standard deviation above or below the mean were carried out. The four components of the Visual Search Task were the dependent variables and weight-status and either GFCQ-T, BIS-11, Delay Discounting or SSRT score the independent variables. So, for each measure of impulsivity or craving, four moderated re- 
gressions were carried out: one for speeded detection of high-caloric food, one for speeded detection of low-caloric food, one for increased distraction by high-caloric food, and one for increased distraction by low-caloric food. In all analyses, age, gender, test location, DEBQ Dietary Restraint scores, DEBQ External Eating scores, presence of binge episodes, daytime activities, living situation, education level, and family income were entered as covariates in the first block. Non-significant variables were omitted from the model. In the second block, group (healthy-weight versus obese) and BIS-11 score, GFCQ-T score, Delay Discounting (AUC), or SSRT were added. In the third and final block, the interaction between the two variables of the second block was entered. Variables were centred prior to entering them in the regression model. As indicated by Variance Inflation Factors (always close to 1) and Tolerance (never below 0.2), there were no multicollinearity problems in any of the conducted regressions. In addition, the Durbin-Watson statistic had a value close to 2 in all analysis, indicating that the assumption of independence of errors was met. P-values below o.05 were considered significant.

\section{RESULTS}

\section{Sample characteristics}

Table 5.1 shows the results of the one-way ANOVA testing for differences between the obese and healthy-weight groups on age, BMI, GFCQ-T, BIS-11, and DEBQ scores and the performance on the Stop Signal Task, Visual Search Task, and Delay Discounting Task. Significant group differences emerged for age, BMI, GFCQ-T, BIS-11, DEBQ External Eating and DEBQ Dietary Restraint, with the people with obesity scoring higher on all these variables. Levene's test showed no homogeneity of variance for BMI $(F(1,317)=97.73, p<.001)$, GFCQ-T $(F(1$, $317)=15.22, p<.001)$, DEBQ External Eating $(F(1,317)=5.27, p=.022)$, DEBQ Dietary Restraint $(F(1,317)=12.30, p=.001)$, and speeded detection of high-caloric food in the Visual Search Task $(F(1,309)=5.33, p=.022)$. Welch $F$ was used for these variables. A Pearson's chi-square test was performed on binge episodes, showing binges to be significantly more common in participants with obesity (46 out of 139 participants reported at least one binge episode in the past 4 weeks) than in healthy-weight participants (binge episodes reported by 7 out of 127 participants), $X^{2}(1)=21.40, p<.001$. Additional Pearson chi-square tests on demographic variables (see Table 5.2) showed differences between the group with obesity and the group with a normal-weight on daytime activities, $X^{2}(4)=25.77, p<.001$, living situation, $X^{2}(5)=$ 
Table 5.1 Means and standard deviations of participant characteristics per group

\begin{tabular}{|c|c|c|c|c|c|c|c|}
\hline & \multicolumn{2}{|c|}{ Participants with healthy weight $(\mathrm{N}=134)$} & \multicolumn{2}{|c|}{ Participants with obesity $(N=185)$} & \multirow[t]{2}{*}{$F$} & \multirow[t]{2}{*}{$p$} & \multirow[t]{2}{*}{ Cohen's d } \\
\hline & M & SD & M & SD & & & \\
\hline Age & 33.04 & 8.15 & 35.19 & 7.59 & 5.90 & .02 & .27 \\
\hline BMI & 22.35 & 1.63 & 38.18 & 6.17 & 1110.72 & .00 & 3.51 \\
\hline GFCQ-T & 46.46 & 14.75 & 65.09 & 20.21 & 90.55 & .00 & 1.05 \\
\hline $\mathrm{BIS}-11^{1}$ & 59.96 & 9.12 & 63.29 & 9.08 & 10.46 & .00 & .37 \\
\hline$D E B Q-E X^{1}$ & 2.79 & 0.51 & 3.13 & 0.62 & 28.52 & .00 & .60 \\
\hline $\mathrm{DEBQ}-\mathrm{DR}{ }^{1}$ & 2.44 & 0.84 & 2.90 & 0.68 & 27.20 & .00 & .60 \\
\hline Delay Discounting ${ }^{2}$ & 0.50 & 0.23 & 0.46 & 0.25 & 2.26 & .13 & - \\
\hline SSRT ${ }^{1}$ & 179.76 & 45.74 & 176.36 & 43.07 & 0.41 & .53 & - \\
\hline VShcds ${ }^{3}$ & -301.86 & 333.78 & -355.90 & 410.49 & 1.63 & .20 & - \\
\hline VSIC ds ${ }^{3}$ & -362.43 & 318.38 & -344.22 & 376.31 & 0.20 & .66 & - \\
\hline VShedi ${ }^{3}$ & 133.36 & 383.26 & 174.02 & 351.78 & 0.94 & .33 & - \\
\hline$V S \mid c d i^{3}$ & 167.56 & 447.70 & 204.75 & 406.39 & 0.58 & .45 & - \\
\hline
\end{tabular}

${ }^{1}$ GFCQ-T = General Food Craving Questionnaire - Trait; BIS-11 = Barratt Impulsiveness Scale - 11; DEBQ = Dutch Eating Behaviour Questionnaire; DEBQ - EX = External Eating subscale of the DEBQ; DEBQ - DR = Dietary Restraint subscale of the DEBQ; SSRT = Stop Signal Reaction Time

${ }^{2}$ Values presented are Areas under the Curve

${ }^{3}$ VS = Visual Search task; hc = high-caloric food items, lc = low-caloric food items, ds = detection speed (lower scores indicate faster detection of food), di = distraction (higher scores indicate more distraction by food)

Table 5.2 Cell count corresponding to Pearson chi-square tests on demographic variables

\begin{tabular}{|c|c|c|c|c|c|c|c|c|c|}
\hline Daytime activities & & Student & Employed & Unemployed & Housewife/man & Other & & & \\
\hline & Healthy-weight & 32 & 77 & 18 & 5 & 2 & & & \\
\hline & Obese & 16 & 132 & 11 & 23 & 3 & & & \\
\hline \multirow[t]{3}{*}{ Living situation } & & Alone & With partner & $\begin{array}{l}\text { With partner } \\
\text { and children }\end{array}$ & $\begin{array}{l}\text { Alone with } \\
\text { children }\end{array}$ & $\begin{array}{l}\text { Alone with } \\
\text { roommates }\end{array}$ & $\begin{array}{l}\text { With } \\
\text { parents }\end{array}$ & & \\
\hline & Healthy-weight & 38 & 37 & 22 & 9 & 21 & 7 & & \\
\hline & Obese & 40 & 36 & 74 & 14 & 7 & 14 & & \\
\hline \multirow[t]{3}{*}{ Educational level'1 } & & None & Primary & VMBO/MAVO & HAVO & VWO & MBO & $\mathrm{HBO}$ & wo \\
\hline & Healthy-weight & 1 & 3 & 7 & 9 & 14 & 16 & 39 & 45 \\
\hline & Obese & 0 & 1 & 32 & 14 & 14 & 68 & 42 & 14 \\
\hline \multirow[t]{3}{*}{ Personal income } & & $<€ 1000$ & $€ 1000-1500$ & $€ 1500-2000$ & $€ 2000-2500$ & $€ 2500-3000$ & $>€ 3000$ & & \\
\hline & Healthy-weight & 53 & 28 & 26 & 19 & 5 & 3 & & \\
\hline & Obese & 55 & 49 & 41 & 22 & 9 & 9 & & \\
\hline \multirow[t]{3}{*}{ Family income } & & $<€ 1000$ & $€ 1000-1500$ & $€ 1500-2000$ & $€ 2000-2500$ & $€ 2500-3000$ & $>€ 3000$ & & \\
\hline & Healthy-weight & 28 & 18 & 19 & 16 & 21 & 32 & & \\
\hline & Obese & 12 & 27 & 33 & 38 & 27 & 48 & & \\
\hline
\end{tabular}

$1 \mathrm{VMBO} / \mathrm{MAVO}, \mathrm{HAVO}$ and VWO are forms of secondary education, with VMBO/MAVO being the lowest level and VWO the highest level. MBO, $\mathrm{HBO}$ and WO are forms of further education, corresponding to the three types of secondary education. $\mathrm{HBO}$ corresponds to college in the US, and WO to university in the US. 
31.30, $p<.001$ highest level of education $x^{2}(8)=63.56, p<.001$, and family income, $x^{2}(5)=$ $17.17, p=.004$, but not personal income, $x^{2}(5)=5.47, p=.36$.

\section{Hypothesis: Higher levels of impulsivity and trait food cravings are predictive of a stronger attention bias, especially in participants with obesity}

\section{Impulsivity - BIS-11}

Detection of high-caloric food. None of the covariates were significant, and they were therefore not retained in the model. The analysis showed a significant interaction between weight group and BIS-11 score, $\beta=-.18, t(310)=2.05, p=.041, P=.013$, indicating that participants with obesity and with a healthy-weight differed in their detection speed of high-caloric food, depending on their degree of impulsivity. More specifically, within the high-impulsive participants, the participants with obesity were significantly faster at detecting high-caloric food than the healthy-weight participants, $\beta=-.19, t(310)=2.32, p=.021$. There was no difference within the low-impulsive participants, $\beta=.04, t(310)=.52, p=.60$. Within the participants with obesity and within the healthy-weight participants, there was no effect of impulsivity (obese: $\beta=-.10, t(310)=1.34, p=.18$; healthy-weight: $\beta=.14, t(310)=1.55, p=.122$ ). Simple slopes and interactions with standardized regression coefficients for participants scoring high (+ 1 SD) and low (-1 SD) on impulsivity are plotted in Figure 5.1. Results of this regression analysis can be found in Table 5.3.

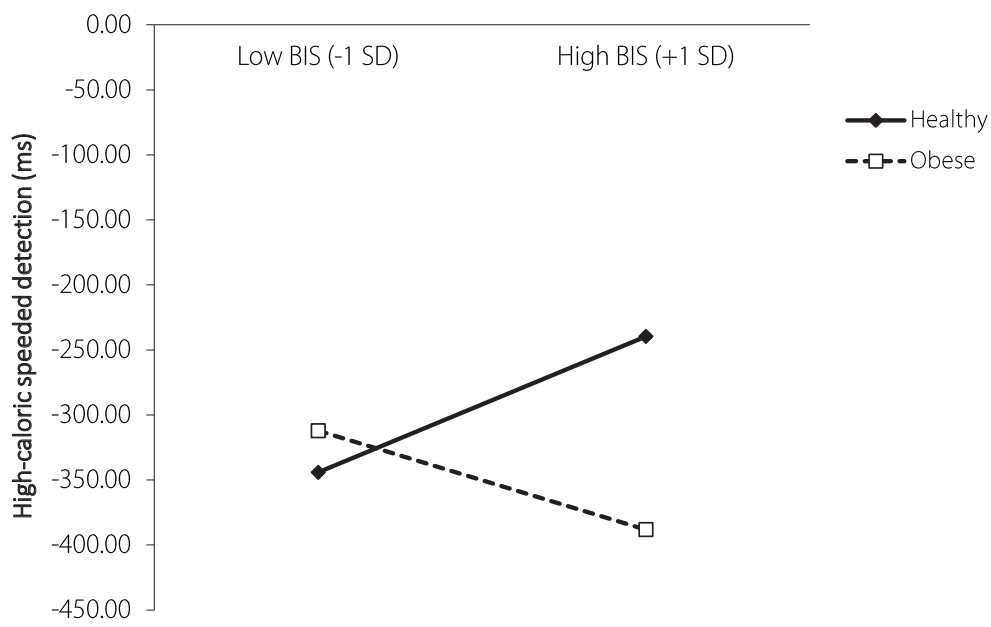

Figure 5.1 Detection speed of high-caloric food as a function of weight and BIS-11 impulsivity scores (-1 SD and +1 SD from the mean BIS-11 score, respectively). Lower scores indicate faster detection of food stimuli 
Table 5.3 Results of moderated regression analysis of weight group and BIS-11 scores on speeded detection of high-caloric food

\begin{tabular}{|c|c|c|c|c|}
\hline \multirow{3}{*}{ Steps and variables ${ }^{\dagger}$} & \multicolumn{4}{|c|}{ High-cal speeded detection } \\
\hline & \multicolumn{2}{|c|}{ Step 1} & \multicolumn{2}{|c|}{ Step 2} \\
\hline & B & SE & B & SE \\
\hline \multicolumn{5}{|l|}{ Location^ } \\
\hline Weight group & -53.90 & 44.59 & -58.25 & 44.41 \\
\hline BIS-11 & -0.04 & 2.37 & 5.66 & 3.65 \\
\hline Weight group X BIS-11 & & & $-9.78^{*}$ & 4.78 \\
\hline$R^{2}$ & \multicolumn{2}{|c|}{.005} & \multicolumn{2}{|c|}{.018} \\
\hline$\Delta R^{2}$ & \multicolumn{2}{|c|}{.005} & \multicolumn{2}{|c|}{$.013^{*}$} \\
\hline
\end{tabular}

${ }^{+}$Note: $\mathrm{B}$ is the unstandardized regression coefficient

$\wedge$ No covariate was included in the regression for high-cal speeded detection, because they did not have a significant effect

* $p<.05$

Detection of low-caloric food. Location was a borderline significant ( $p=.056$ ) covariate and was therefore retained in the model. There was no interaction between BIS-11 scores and weight group, $\beta=-.16, t(310)=1.77, p=.077$, indicating no role of trait impulsivity in the relationship between weight status and the detection speed of low-caloric food items.

Distraction by high- and low caloric food. There was no significant interaction between weight status and BIS-11 score for distraction by either high-caloric, $\beta=.035, t(310)=.46, p=$ .647 , or low-caloric food, $\beta=.092, t(310)=1.22, p=.224$. These findings indicate no effect of self-reported impulsivity and weight status on being distracted by food.

\section{Impulsivity - SSRT}

The weight status X SSRT interaction was non-significant for detection of high-caloric food, $\beta=-.037, t(283)=-.47, p=.639$, detection of low-caloric food, $\beta=.077, t(283)=.98, p=.329$, distraction by high-caloric food, $\beta=.008, t(283)=.10, p=.922$, or distraction by low-caloric food, $\beta=-.002, t(283)=-.024, p=.981$. In sum, there was no effect of weight status and SSRT on an attention bias for food. 


\section{Impulsivity - Delay Discounting}

We found no significant interaction between weight status and Delay Discounting scores for detection of high-caloric food, $\beta=.134, t(309)=1.44, p=.151$, detection of low-caloric food, $\beta=.019, t(309)=.207, p=.836$, distraction by high-caloric food, $\beta=.083, t(309)=.89$, $p=.373$, or distraction by low-caloric food, $\beta=.134$, t(309) $=1.44, p=.151$. Taken together, these results indicate no influence of reward sensitivity and weight status on attention bias for food.

\section{Trait Craving - GFCQ-T}

There were non-significant interactions between weight status and GFCQ-T scores for detection of high-caloric food, $\beta=.061, t(310)=.80, p=.426$, detection of low-caloric food, $\beta=.027, t(310)=.35, p=.729$, distraction by high-caloric food, $\beta=-.029, t(310)=-.38, p=.702$, and distraction by low-caloric food, $\beta=-.004, t(310)=-.05, p=.963$. These results show that weight status and trait food craving do not influence attention bias for food.

\section{DISCUSSION}

Results of the present study showed that trait impulsivity moderated the relationship between obesity and attention bias: high-impulsive participants with obesity were significantly faster than high-impulsive healthy-weight participants in detecting a high-caloric food item among neutral items. All other analyses, including those using the other two measures of attentional bias (i.e. distraction by high and low caloric foods) and other measures of impulsivity (i.e. stop-signal-task, delay discounting task) or trait craving did not yield significant results.

These results point out that in our participants with obesity an impulsive personality is associated with a speedy detection of high-caloric food, while this is not the case in healthy-weights. High calorie foods are more salient and more rewarding for people with obesity than for healthy-weights (Stice, Spoor, Bohon, Veldhuizen, \& Small, 2008; Stoeckel et al., 2008) and therefore they might more easily draw the attention of impulsive people with obesity. In healthy-weight impulsive people, immediate pleasure might be derived from other sources than palatable food. A preference for high-caloric food seems to be less pronounced in the healthy-weight population (Ransley et al., 2003; Stoeckel et al., 2008), and an additional lack of impulsivity might lower such a preference even more. 
Remarkably, we only found these effects with self-reported trait impulsivity and not when impulsivity was measured with behavioural tasks. Whereas trait impulsivity refers to a general impulsive personality, behavioural tasks measure only one specific state-like aspect of impulsivity, in this case response inhibition or sensitivity to immediate reward. Apparently, these specific aspects of impulsivity do not influence attention for food, while being a generally impulsive person does. However, exactly why this would happen remains unclear. Perhaps the specificity of the behavioural tasks limits generalization to other behaviours (Reynolds et al., 2006). Furthermore, impulsivity questionnaires do not fully reflect the behavioural measures and can therefore not be assumed to measure the same. The results do add to a growing body of literature demonstrating no or weak correlations between different self-report and behavioural measures of impulsivity (Enticott, Ogloff, \& Bradshaw, 2006; Marsh, Dougherty, Mathias, Moeller, \& Hicks, 2002; Reynolds et al., 2006), thereby stressing the multidimensionality of the impulsivity construct. In line with our results, Hou et al. (2011) found a correlation between self-reported trait impulsivity and attentional bias for food cues. It thus seems that self-reported trait impulsivity is a more critical factor than response inhibition or reward sensitivity in understanding attention biases for food cues. These findings on food attentional bias differ from substance-related biases however: A recent meta-analysis showed a significantly stronger relationship between behavioural measures of impulsivity and substance-related attentional bias than between self-reported trait measures and such an attentional bias (Coskunpinar \& Cyders, 2013). Without doubt, impulsivity is a complicated construct that can be conceptualized in many ways (Dawe \& Loxton, 2004). It remains a question for future research to see whether a different conceptualization would lead to different results. Likewise, measures of attentional bias vary across studies as well, using for example dot-probe vs. visual search tasks, food words vs. food pictures, and some including eye-tracking measurements. It cannot be ruled out that alternative measures of attention bias would have yielded different results.

Another noteworthy finding related to the three types of impulsivity measures is that we did show higher impulsivity in participants with obesity compared to healthy-weight participants on self-reported trait impulsivity, but not on behavioural tasks. Our findings on self-report are in concordance with previous studies (Fassino et al., 2002; Mobbs et al., 2010; Rydén et al., 2003), whereas the findings on response inhibition (Hendrick, Luo, Zhang, \& Li, 2011; Nederkoorn et al., 2006) and delay discounting are mixed (Nederkoorn et al., 2006; Weller, Cook, Avsar, \& Cox, 2008; Yeomans, Leitch, \& Mobini, 2008). If and how weight is related to effective response inhibition and reward sensitivity are questions that remain to be answered. 
The absence of any findings on the influence of trait craving on attention bias is surprising, as studies including trait chocolate cravers have found both increased distraction by and speeded detection of chocolate cues (Kemps \& Tiggemann, 2009; Smeets et al., 2009). Future studies might test whether trait craving for a specific food in combination with an attention bias paradigm involving that particular food elicits stronger effects than general trait food craving with an attention task consisting of different types of tasty foods, as was the case in the present study.

Finally, we would like to note that trait impulsivity is linked to the dopaminergic system. More specifically, a recent imaging study showed that trait impulsivity is negatively associated with the expression of midbrain dopamine $D_{2 / 3}$ autoreceptors and with amphetamineinduced release of endogenous striatal dopamine (Buckholtz et al., 2010). In addition, we and others showed loss of striatal $D_{2 / 3}$ receptors in obesity (De Weijer et al., 2011; Van de Giessen, Celik, Schweitzer, Van den Brink, \& Booij, 2014; Wang et al., 2001), which are located predominantly post-synaptically. Taking into account these results as well as the results of the present study, it would be interesting to evaluate in future studies whether impulsive patients with obesity, compared to non-impulsive patients with obesity, show loss of expression of midbrain dopamine $\mathrm{D}_{2 / 3}$ autoreceptors as well as an increased striatal dopamine release, and whether this is also related to increased attentional bias for high-caloric food.

This study has some limitations. First, there was an overrepresentation of (former) students in the normal-weight group. Although the participants were matched on age and gender, differences between groups emerged on other demographic variables, namely level of education, living situation, daytime activities, and family income. A second limitation of the present study concerns the absence of hunger and craving measures before administration of the visual search paradigm, as previous studies have indicated that these could be influential variables (see for example Castellanos et al. (2009) and Werthmann et al. (2011)). However, all participants were instructed to eat something small two hours before the start of the experiment to standardize hunger levels. Third, because information on construct validity was not available for all used questionnaires, we cannot be certain whether the constructs used were adequately measured. However, all questionnaires are among the most well-known and commonly used in the field, ensuring the possibility of comparing our results to those of other studies.

Finally, it is important to note that only one of the measures yielded significant results, and that the effects obtained by this measure were quite small. It is therefore too early to draw firm conclusions about the role of impulsivity in attentional bias for food. However, if the results of the present study can be replicated, they could be of relevance for clinical 
practice, as they underline the role of self-reported impulsivity in the relationship between weight status and attentional bias for food cues. Of most clinical relevance is the finding that high impulsive participants with obesity were faster at detecting high caloric foods than their low impulsive counterparts, suggesting that impulsivity should be taken into account as an additional factor in the treatment of obesity or overweight in clinical practice. In addition, attention retraining in which patients are trained to avoid or disengage attention from food cues could be useful to reduce the attention-grabbing powers of food in this particular group of high impulsive people with obesity. Studies in groups of heavy drinkers (Schoenmakers, Wiers, Jones, Bruce, \& Jansen, 2007), abstinent alcohol-dependent patients (Schoenmakers et al., 2010; Wiers, Eberl, Rinck, Becker, \& Lindenmeyer, 2011), smokers (Attwood, O'Sullivan, Leonards, Mackintosh, \& Munafò, 2008), and concerning food intake (Werthmann et al., 2014) have yielded promising results in this regard.

In conclusion, trait impulsivity is associated with increased attentional bias for high calorie food in people with obesity, but not in people with a healthy weight. This characteristic might make it more difficult for impulsive overweight people to live in a society with an overwhelming presence of high-caloric food.

\section{ACKNOWLEDGEMENTS}

This study was partly made possible by financial support of the Netherlands Organisation for Scientific Research (NWO): Vici Grant 453.10.006, awarded to Anita Jansen. The authors would like to thank Nicolette Siep, Bart van den Boogard, Mieke Dorssers, Melanie de Wit, Brit Giesbertz, Linda Hermens, and Michelle de Haan for their assistance in conducting the study. The authors would also like to thank Co-Eur, centre for obesity and eating disorders, for their help in the recruitment and testing of participants. 



\section{6}

\section{Emotional eating and Pavlovian learning:}

Does negative mood facilitate appetitive conditioning? 


\section{ABSTRACT}

Objective: Emotional eating has been suggested to be a learned behaviour; more specifically, classical conditioning processes might be involved in its development. In the present study we investigated whether a negative mood facilitates appetitive conditioning, and whether trait impulsivity influences this process. Method: After undergoing either a negative or neutral mood induction, participants were subjected to a differential classical conditioning procedure, using neutral stimuli and appetizing food. Two initially neutral distinctive vases with flowers were (CS+) or were not (CS-) paired with chocolate mousse intake. We measured participants' expectancy and desire to eat (4 CS+ and 4 CS- trials), salivation response, and actual food intake. The BIS-11 was administered to assess trait impulsivity. Results: In both mood conditions, participants showed a classically conditioned appetite. Unexpectedly, there was no evidence of facilitated appetitive learning in a negative mood with regard to expectancy, desire, salivation, or intake. However, immediately before the taste test, participants in the negative mood condition reported a stronger desire to eat in the CS+ compared to the CS- condition, while no such effect occurred in the neutral group. An effect of impulsivity was found with regard to food intake in the neutral mood condition: high-impulsive participants consumed less food when presented with the CS+ compared to the CS-, and also less than low-impulsive participants. Discussion: An alternative pathway to appetitive conditioning with regard to emotions is that it is not the neutral stimuli, but the emotions themselves that become conditioned stimuli and elicit appetitive responses.

Keywords: Emotional eating; Appetitive conditioning; Mood; Impulsivity; Cue reactivity. 


\section{INTRODUCTION}

Emotional eating (i.e., eating in response to negative emotions) is a contributing factor in obesity and eating disorders (Hays \& Roberts, 2008; Polivy \& Herman, 2002). Several studies have shown overeating after the experience of negative emotions, such as stress, anxiety or sadness, in dieters (Cools, Schotte, \& McNally, 1992; Heatherton, Herman, \& Polivy, 1991; Loxton, Dawe, \& Cahill, 2011; Wallis \& Hetherington, 2004), obese people (Patel \& Schlundt, 2001; Schneider, Appelhans, Whited, Oleski, \& Pagoto, 2010), and obese binge eaters (Agras \& Telch, 1998; Chua, Touyz, \& Hill, 2004). However, it is unknown how emotional eating originates or how it is maintained.

It has been suggested that emotional eating is a learned behaviour, as the natural response for most people in the face of adversity is to decrease food intake (Wardle, 1990). Specifically, classical or Pavlovian conditioning has been proposed to be the involved mechanism (Greeno \& Wing, 1994; Jansen, 1998; Jansen, Havermans, \& Nederkoorn, 2011; Wardle, 1990). In classical conditioning, food intake is regarded as an unconditioned stimulus (US), eliciting unconditioned physiological responses (URs), such as insulin release, blood sugar increase and salivation. Over time, stimuli that are systematically paired with food intake can start to predict intake, thereby becoming conditioned stimuli (CS). After learning that the CS predicts the occurrence of the US, CSs are capable of eliciting appetitive responses, such as a conditioned desire to eat (i.e., cue reactivity). Virtually any stimulus in the environment can become a food-signaling CS, including the sight or smell of food, a certain time of the day, the environment or context (Jansen, 1998; Wardle, 1990) and it is also suggested that emotions can become food-signaling CSs (Jansen, Havermans, \& Nederkoorn, 2011).

Experimental studies indeed show that eating desires can be conditioned quite easily. Van Gucht and colleagues (Van Gucht, Baeyens, Hermans, \& Beckers, 2013; Van Gucht, Baeyens, Vansteenwegen, Hermans, \& Beckers, 2010; Van Gucht, Vansteenwegen, Beckers, \& Van Den Bergh, 2008a; Van Gucht, Vansteenwegen, Van den Bergh, \& Beckers, 2008b) and Papachristou, Nederkoorn, Beunen, and Jansen (2013) convincingly demonstrated appetitive conditioning to a neutral cue (i.e., a serving tray) in several studies, with participants reporting increased food expectancy, eating desire, and automatic approach tendencies when presented with the cue that predicted the intake of appetizing foods (CS+; a tray in one shape and colour) compared to the cue that did not predict the intake of appetizing foods (CS-; a tray in another shape and colour). Van den Akker, Jansen, Frentz, and Havermans (2013) extended these findings by showing appetitive conditioning in response to contexts (neutral environments in a virtual reality lab), indicating that besides specific 
cues, specific contexts can also come to elicit food expectancy and eating desire after the context becomes a predictor of intake. In addition to expectancy and desire, Van den Akker et al. (2013) also measured salivation and actual food intake. They found increased saliva production in participants presented with the CS+, but not the CS- context. Thus, after only 6 pairings of a neutral context with the intake of appetizing food, some evidence was found that participants salivated more in the context that predicted the intake compared to a context that did not predict intake. The authors also found that food intake increased in the CS+ compared with the CS- contexts, but only for participants high in impulsivity. This effect of impulsive people showing increased conditioned food intake is interesting, considering the wealth of research showing a positive association between impulsivity on the one hand and food consumption and obesity on the other hand (see Guerrieri, Nederkoorn, \& Jansen, 2008 for an overview; Velázquez-Sánchez et al., 2014). Importantly, a role for impulsivity in appetitive conditioning was proposed by Gray (Corr, Pickering, \& Gray, 1995) in his BIS/BAS theory, which predicted that high impulsivity (i.e., strong BAS; Behavioural Approach System) is related to increased associative appetitive learning, while high anxiety (i.e., strong BIS; Behavioural Inhibition System) is related to increased aversive learning. Research with regard to the facilitating role of impulsivity in appetitive learning has however yielded mixed results (Corr, 2004; Corr et al., 1995; Paisey \& Mangan, 1988; Papachristou et al., 2013; Van den Akker et al., 2013; Zinbarg \& Mohlman, 1998; Zinbarg \& Revelle, 1989) and studies specifically on food as the appetitive stimulus are scarce (Papachristou et al., 2013; Van den Akker et al., 2013).

If emotional eating is, as we suggest, learned through classical conditioning, two pathways through which this could occur are most obvious. First, it is frequently reported by emotional eaters that they overeat when feeling bad. If a particular emotional state is frequently associated with the intake of appetizing high calorie foods, and the contingency between this emotion and eating is strong (i.e., the probability of the emotion leading to eating approaches one), this association will ultimately lead to the emotion becoming a reliable predictor of food intake, i.e., a CS. After conditioning, confrontation with the CS (emotion) will elicit cue reactivity, i.e., an eating desire or food craving, and ultimately food intake. An alternative pathway is negative mood facilitating the learning of associations between neutral stimuli and food intake. High-calorie foods have strong rewarding properties (see for example Coletta et al., 2009; Macht \& Dettmer, 2006; Macht \& Mueller, 2007; Small, Zatorre, Dagher, Evans, \& Jones-Gotman, 2001), which might be extra rewarding and thus relevant for people who find themselves in a negative emotional state (Baker, Piper, McCarthy, Majeskie, \& Fiore, 2004; Hepworth, Mogg, Brignell, \& Bradley, 2010). They would benefit 
from learning that certain cues predict food intake that might alleviate their bad mood. In this model, a neutral stimulus from the environment, or the environment itself, becomes the CS. The CS predicts food intake and as a consequence of the rewarding foods, a better mood. This pattern might be considered a specific form of state-dependent learning or context learning, in which the negative mood functions as a state or context. Furthermore, research in fear conditioning has brought forward some evidence that negative contexts (i.e., threat) facilitate conditioning (Karos, Meulders, \& Vlaeyen, 2014). The latter pathway is the one under investigation in the current study.

In the present study, we tested the hypothesis that participants in a negative mood would show facilitated appetitive conditioning. We expected stronger differential responses to the CSs for the group that was conditioned in a negative mood compared to the group that was conditioned in a neutral mood: those in a negative mood would show a larger difference in expectancies to receive food, desires to eat food, saliva production, and food intake in response to the CS predicting the intake of high-caloric food (CS+) than to the CS not predicting food intake (CS-). Because impulsivity is strongly related to overeating and obesity and earlier research shows that highly impulsive participants in particular eat more after classical conditioning (Van den Akker et al., 2013), we investigated the possibility of a moderating effect of impulsivity on expectancy, desire, salivation and food intake, with higher levels of impulsivity facilitating conditioning.

\section{METHOD}

\section{Participants}

A total of 127 female undergraduate students, aged between 17 and 30 years $(M=19.98$, SD = 1.79), took part in the study. The students were told that the study concerned examining the relationship between music and taste. To be included, participants had to like chocolate mousse (the food of choice in the current study; scoring at least 3 on a 5-point Likert scale ranging from 'I do not like chocolate mousse at all' (1) to 'I really like chocolate mousse' (5)), which was assessed with a single question among four filler questions during sign up. They were instructed to eat something small (e.g., a sandwich or an apple) 2 hours before the start of the experiment, and to refrain from food intake (with the exception of water) in the time thereafter. Furthermore, they were asked not to eat any chocolate in the 24 hours before participating. For participation, participants received either one course credit, or $€ 7.50$. The study's procedure was approved by the local ethics committee. At the end 
of the study, when all testing was completed, participants received a debriefing via email containing an explanation of the aims and measures of the study.

\section{Design}

The study used a mixed design. Participants were randomly divided over 2 conditions: negative mood and neutral mood (from hereon called Negative and Neutral, respectively). Within these conditions, participants were divided into two groups for the post-conditioning saliva measurement and taste test. Half of the group in each mood condition received these measurements in presence of the CS+ (from here on called 'Negative CS+' and 'Neutral CS+'), while the other half received them in presence of the CS- (from here on called 'Negative CS-' and 'Neutral CS-'). Mood, expectancy to receive food and desire to eat were within-subject variables, measured 8 times for each participant (4 times in response to CS+, 4 times in response to (S-). Saliva production was an additional within-subject variable, measured at baseline and at the end of the conditioning procedure (for half the participants in presence of the CS+, for the other half in presence of the CS-).

\section{Stimuli}

Mood manipulation. After sign-up, the participant was instructed to bring at least three pieces of music (lasting for a minimum of 8 minutes) that made her happy and three pieces of music that made her sad (also totalling at least 8 minutes) with her to the lab. Participants were randomly assigned to either the neutral or negative condition. To induce a negative mood, the participant was asked to think of a sad event that happened to her. When she had something in mind, the sad songs chosen by the participant were played and she was given a blank sheet of paper and asked to write down that memory as detailed as possible. If she finished writing before the experimenter returned, she was instructed to keep thinking about the event she had written about. She was told that her memory would not be read by the experimenter. All stories were anonymously checked by an independent rater (A.J.) however, and this check confirmed that all participants had followed the instructions. In the neutral mood procedure, the participant was asked to write down the route she travelled from her home to the university library, and when she got there, which steps she would need to copy pages from a book. In the neutral condition, this assignment was accompanied by the musical piece De l'aube à midi sur la mer by Claude Debussy (playing time: 8 min $53 \mathrm{sec})$. Vuoskoski and Eerola (2012) reported that personally relevant sad music combined with the recollection of sad autobiographical memories is an effective way of inducing sad moods. In addition, they found De l'aube à midi sur la mer did not change 
participant's emotions. Total duration of the mood manipulation was 8 minutes. Shorter versions of the mood manipulation were repeated after trials 4 and 7 of conditioning, to ensure the participant remained in the required mood. During these in-between manipulations, participants continued writing down or thinking about their memory for 1 minute, while the sad or neutral music played. The personally selected pieces of happy music were only used at the end of the experiment, to counteract possible lingering negative feelings.

US and CSs. Plastic teaspoons with approximately 2 grams of chocolate mousse (Almhof) were used as USS. A round green vase with three artificial red roses and a rectangular orange vase with three artificial white daisies were used as CSs. One of these vases served as CS+ and the other as CS-, and this was counterbalanced across participants. Four CS+ and four CS- trials were presented to each participant. The order of the trial presentation was random, with the restriction that the first two trials always consisted of one CS+ and one CS-, counterbalanced across participants. Furthermore, there were no more than two consecutive trials of the same type (CS+ or CS-).

\section{Measures}

Hunger. Hunger was measured before and after the mood manipulation by means of a 100 mm Visual Analogue Scale (VAS). Participants had to answer the question 'How hungry are you at this moment?', with the left anchor of the scale corresponding to 'not hungry at all', and the right anchor to 'very hungry'.

Expectancy and desire. Expectancy to receive chocolate mousse and desire to eat chocolate mousse were assessed with $100 \mathrm{~mm}$ VASs. Expectancy was measured with the question 'How strongly do you expect to receive chocolate mousse at this moment?', with the scale ranging from 'no expectancy at all' to 'very strong expectancy'. For desire, the question was 'If you concentrate on the vase with flowers, how strong is your desire to eat chocolate mousse?' Ratings ranged from 'no desire at all' to 'very strong desire'. Identical desire-VAS's were administered before and after the mood manipulation (for eating in general), and before the taste test (specifically aimed at eating chocolate mousse).

CS liking. Liking for the CS was measured in two different ways. Upon the first encounter with each CS, participants filled out a VAS scale asking 'How pleasant do you find this vase with flowers?', with answers ranging from 'not pleasant at all' to 'very pleasant'. This question was repeated for both CSs after the last conditioning trial. In addition, participants were 
asked to imagine that they were allowed to take one of the flower vases home, and they had to indicate which of the two they preferred.

Contingency awareness. To check whether participants were aware of the contingency between CS+ and chocolate mousse consumption, they were asked 'Was there a specific vase with flowers after which you were always asked to eat chocolate mousse?' If participants answered this question with 'yes', they were asked to indicate which vase was always followed by food intake. Other answering options were 'no' and 'I don't know'.

Saliva. Saliva production was measured with 2.5 cotton dental rolls (Hartmann Celluron no. 2), which were weighed beforehand on a kitchen scale (0.01 g precision) and presented to the participant in a small plastic bag. She placed the two whole rolls between her cheek and lower gum on each side of her mouth, and an additional half dental roll under her tongue. The rolls were removed after one minute, replaced in the bag, and weighed again.

Taste test and food intake. The taste test consisted of 3 identical small bowls each filled with approximately $74.13(\mathrm{SD}=5.97)$ grams of chocolate mousse. The bowls were brought into the testing room by the experimenter, together with either the CS+ or CS- vase with flowers (which was counterbalanced across participants), and placed on the table in front of the participant. She was told there were some small differences between the three types of mousse that she had to detect (in fact they were all identical and the same as the mousse used during conditioning), in order to stimulate her to really try and taste from all the bowls. She received a short questionnaire regarding taste and structure of the three bowls of chocolate mousse, and was told she had 5 minutes to answer the questions, and that the experimenter would return once that time had passed. Unbeknownst to the participant, the bowls were weighed before and after the taste test to assess chocolate mousse consumption.

Chocolate mousse liking. Three of the questions incorporated in the taste test questionnaire measured liking of the chocolate mousse. For each of the three bowls, participants rated the mousse on a 5-point Likert scale, ranging from 'I do not like this mousse at all' to 'I like this mousse very much'. The remaining questions were not used for analyses.

Mood. Two $100 \mathrm{~mm}$ VAS's were used to assess mood, one measuring sadness and one measuring happiness. They stated 'how sad/happy do you feel at this moment?' and ranged 
from 'not at all' to 'very much.' In addition, a similar question regarding boredom was administered to be able to check whether boredom during conditioning possibly influenced the sad and happy mood ratings.

$D E B Q$ (EE and DR). The Emotional Eating (EE) and Dietary Restraint (DR) subscales of the Dutch Eating Behaviour Questionnaire (DEBQ; Van Strien, 2005) were used to measure emotional eating and dietary restraint. The subscales consist of 13 and 10 items, respectively, and answers are given on a five-point Likert scale ranging from 'never' to 'very often'. Final scores on each DEBQ subscale range from 1 to 5 , with higher scores pointing to a higher degree of emotional eating or dietary behaviour.

BIS-11. The Barratt Impulsiveness Scale - 11 (Patton, Stanford, \& Barratt, 1995) measures impulsivity through a 30-item self-report questionnaire. Items have to be answered on a 4-point scale, with higher total scores reflecting higher levels of impulsivity. Only total score on the BIS-11 was included in analyses. Patton et al. (1995) reported that the BIS-11 has high internal consistency.

Compliance to eating instructions. Participants were asked to report what and when they had last eaten before participating in the experiment. In addition, they were asked whether they had consumed any chocolate in the past 24 hours, and if yes, when exactly this had occurred.

\section{Procedure}

Upon arrival in the laboratory, the participant was seated at a table and signed an informed consent form. A baseline saliva measure was conducted, and she then filled out VASs concerning mood, hunger and desire to eat. This was followed by either the neutral or negative mood manipulation, and then again the mood, hunger and desire to eat VASs. At this point, the conditioning procedure started. The participant was informed that she would now be presented with vases with flowers, and that one of the vases would always be followed by something to eat, while the other one would not. The first vase was then taken from a large bag hidden underneath the table and placed on the table in front of the participant. She was instructed to concentrate on the vase and flowers for 10 seconds, and then received the VASs for desire, expectancy, and CS liking. From a closed box placed on the table, the experimenter then took out a small cup containing either a spoon filled with chocolate mousse or an empty spoon, depending on whether the vase was determined to be CS+ 
or CS-. In the case of CS+, the participant was instructed to eat the chocolate mousse. In case of the CS-, the experimenter told her that because the spoon was empty, no eating would occur. The spoon and vase were placed back in the box and bag respectively, and a 45-second intertrial interval followed, during which the participant received the mood VASs to fill out. This sequence of events was repeated randomly 8 times, 4 times with a CS+ and 4 times with a CS-. After trials 4 and 7, one-minute mood manipulations took place. During trial 8, after being presented with the CS+ or CS- and filling out the VASs for expectancy and desire, but before receiving (or not receiving) chocolate mousse, a second saliva measurement was performed. After finishing the conditioning trials, the participant filled out the questions regarding CS liking. The experimenter then brought in the bowls of chocolate mousse for the taste test together with the CS+ or CS-. The participant rated her desire to eat the mousse, and was left alone to taste. The chocolate mousse was taken away after 5 minutes and the participant filled out the contingency VAS. The participant then filled out the DEBQ, BIS-11 and questions regarding compliance to eating instructions while listening to her selected happy songs. Finally, her height and weight were measured wearing street clothes and no shoes, and she was reimbursed for participation.

\section{Statistical analyses}

Six participants were excluded because they did not report contingency awareness, as there is a wide consensus among conditioning researchers that contingency learning and contingency awareness are necessary for conditioning to occur (Boddez, et al., 2013; Lovibond \& Shanks, 2002). One additional participant was excluded for not bringing her own music to the lab. With regard to chocolate intake, 115 participants complied with the instructions and had not eaten chocolate for 24 hours, 4 participants had eaten chocolate but on the previous day, and one participant reported eating chocolate on the day of the experiment. We reanalysed our data excluding the 5 participants who did not comply with our instructions. Because this did not change our results, we reported analyses based on all 120 participants, with the exception of analyses including saliva measurements, which were performed on 118 participants due to missing data.

To investigate the effect of the mood manipulation, a 2 (Condition: negative vs. neutral) $X$ 10 (Time: baseline, post-manipulation, 8 measurements during conditioning) mixed ANOVA was conducted. To control for dietary restraint, and to check for influences of impulsivity and emotional eating on acquisition, DEBQ emotional eating, DEBQ dietary restraint, and BIS-11 scores were included as covariates in all following ANCOVAs. Given that there were no a priori hypotheses with regard to emotional eating and dietary restraint, a was adjusted to 
.008 (for analyses on expectancy and desire) or .016 (for all other analyses) to correct for the number of interactions tested among these variables.

Differential acquisition of expectancy and desire to eat chocolate mousse was measured through mixed 2 (Condition: negative vs. neutral) X 2 (CS-type: CS + vs. CS-) X 4 (Trial: 1, 2, 3, 4) ANCOVAs. Changes in liking of the CS's were investigated by a 2 (Condition: negative vs. neutral) X 2 (CS type: CS+ vs. CS-) X 2 (Time: baseline, post-conditioning) mixed ANCOVA. A binomial test was used to assess preference of CS+ over CS-. A 2 (Condition: negative vs. neutral -) X 2 (CS-type: CS + vs. CS-) X 2 (time: baseline, trial 4) mixed ANCOVA was conducted to analyse saliva production. With regard to all repeated measures analyses, GreenhouseGeisser corrections are reported when sphericity was violated.

Desire to eat immediately before the taste test and food intake (in grams) were first analysed in a 2 (Condition: negative vs. neutral) X 2 (CS-type: CS+ vs. CS-) ANCOVA. Subsequently we performed moderated regression analyses with simple slope testing and spotlightanalyses at one standard deviation above or below the mean of the BIS-11 for the neutral and negative conditions separately, to investigate the moderating role of impulsivity on chocolate mousse consumption. Chocolate mousse intake was the dependent variable and condition and BIS-11 scores the independent variables. In all analyses, DEBQ Emotional Eating scores and DEBQ Dietary Restraint scores were entered as covariates in the first block. In the second block, CS type (CS- vs. CS+ with CS- coded as reference group) and BIS-11 score were added. In the third and final block, the interaction between the two variables of the second block was entered. Variables were centred prior to entering them in the regression model. As indicated by Variance Inflation Factors (always close to 1) and Tolerance (never below 0.2), there were no multicollinearity problems in any of the conducted regressions. In addition, the Durbin-Watson statistic had a value close to 2 in all analysis, indicating that the assumption of independence of errors was met. P-values below 0.05 were considered significant.

\section{RESULTS}

\section{Group characteristics}

As shown in Table 6.1, the participants in the four conditions did not differ on age, BMI, BIS-11 scores, DEBQ emotional eating, DEBQ dietary restraint, baseline mood (i.e., happiness and sadness), minutes since last eaten, or baseline hunger. However, a difference in hunger did emerge after the mood manipulation. Direct comparison between hunger and desire to eat 
Table 6.1 Mean and standard deviation of participant characteristics per condition

\begin{tabular}{|c|c|c|c|c|c|c|c|c|c|c|}
\hline & \multicolumn{2}{|c|}{ Negative $C S+(n=30)$} & \multicolumn{2}{|c|}{ Negative CS- $(n=31)$} & \multicolumn{2}{|c|}{ Neutral $C S+(n=30)$} & \multicolumn{2}{|c|}{$\begin{array}{c}\text { Neutral CS- } \\
(n=29)\end{array}$} & \multirow[b]{2}{*}{$\mathrm{F}$} & \multirow[b]{2}{*}{$p$} \\
\hline & M & SD & M & SD & M & SD & M & SD & & \\
\hline Age & 19.90 & 1.75 & 20.00 & 1.59 & 20.03 & 2.27 & 19.97 & 1.57 & .03 & .99 \\
\hline BMI & 22.04 & 3.04 & 22.35 & 3.18 & 22.70 & 4.34 & 22.25 & 2.65 & .20 & .90 \\
\hline BIS-11 & 62.87 & 9.87 & 61.35 & 10.44 & 63.80 & 8.64 & 64.21 & 10.24 & .50 & .68 \\
\hline DEBQ-EE & 2.61 & .64 & 2.66 & .67 & 2.71 & .57 & 2.98 & .54 & 2.21 & .09 \\
\hline DEBQ-DR & 2.53 & .96 & 2.95 & .73 & 2.87 & .83 & 2.68 & .81 & 1.57 & .20 \\
\hline Sadness & 13.40 & 15.97 & 10.23 & 9.03 & 15.83 & 11.29 & 12.03 & 13.48 & 1.06 & .37 \\
\hline Happiness & 65.07 & 17.32 & 68.13 & 15.92 & 65.30 & 10.66 & 67.59 & 14.07 & .34 & .80 \\
\hline Minutes since eating ${ }^{1}$ & 157.00 & 40.34 & 155.97 & 46.86 & 142.86 & 40.74 & 151.85 & 27.04 & .76 & .52 \\
\hline Hunger baseline & 53.40 & 22.17 & 48.77 & 22.15 & 49.67 & 17.03 & 52.66 & 21.20 & .36 & .79 \\
\hline Hunger after manipulation & 40.03 & 23.35 & 39.58 & 20.02 & 52.87 & 18.82 & 54.03 & 17.48 & 4.64 & .004 \\
\hline Desire baseline & 62.43 & 16.84 & 58.97 & 19.58 & 63.60 & 15.12 & 63.97 & 15.78 & .55 & .65 \\
\hline Desire after manipulation & 41.07 & 19.08 & 40.23 & 21.25 & 60.30 & 16.98 & 58.83 & 17.53 & 10.17 & .000 \\
\hline
\end{tabular}

${ }^{1}$ For the Neutral CS+ and Neutral CS- conditions, analyses are based on the data of 28 and 27 participants, respectively.

in the negative and neutral mood conditions yielded significant differences, $F(1,119)=14.10$, $p<.001$ and $F(1,119)=30.87, p<.001$, respectively.

\section{Mood manipulation}

Condition X Time repeated measures ANOVAs showed that the mood induction was successful, as indicated by significant Condition X Time interactions on both sadness $F(6.08$, $717.64)=52.53, p<.001$ (see Figure 6.1a), and happiness $F(6.91,815.70)=21.49, p<.001$ (see Figure 6.1b).

\section{US expectancy}

With regard to US expectancy, a mixed Condition X CS-Type X Trial ANCOVA revealed a significant CS-type X Trial interaction, $F(2.06,230.76)=180.63, p<.001$, confirming differential acquisition of US expectancy in response to CS+ or CS-. Thus, participants learned to expect food when confronted with the CS+, and to not expect food when confronted with the CS- (see Figure 6.2). There was no influence of impulsivity on differential expectancy over time, $F(2.06,230.76)=.67, p=.52$. The Condition X CS-type X Trial interaction was not significant, $F(2.06,230.76)=.45, p=.64$, indicating no differences in acquisition of US expectancy 

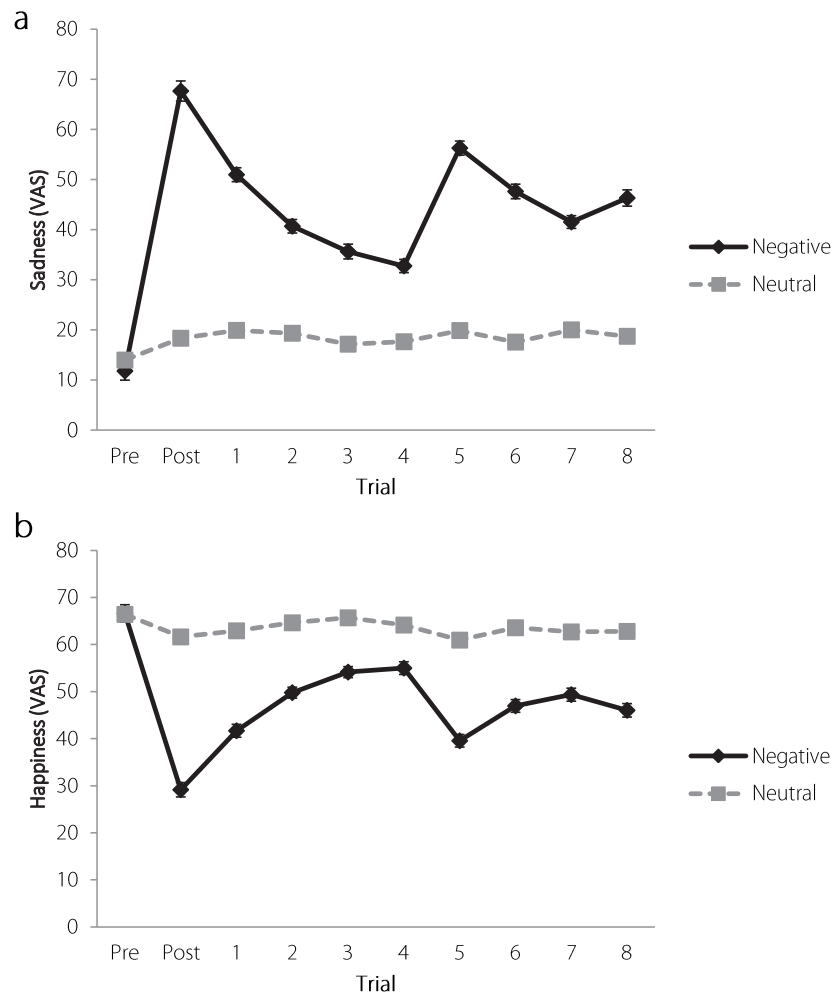

Figure 6.1 Mean sadness (a) and happiness (b) scores (+ SEM) for participants in the negative and neutral conditions at baseline (pre), after mood manipulation (post), and during the conditioning procedure (trial 1-8)

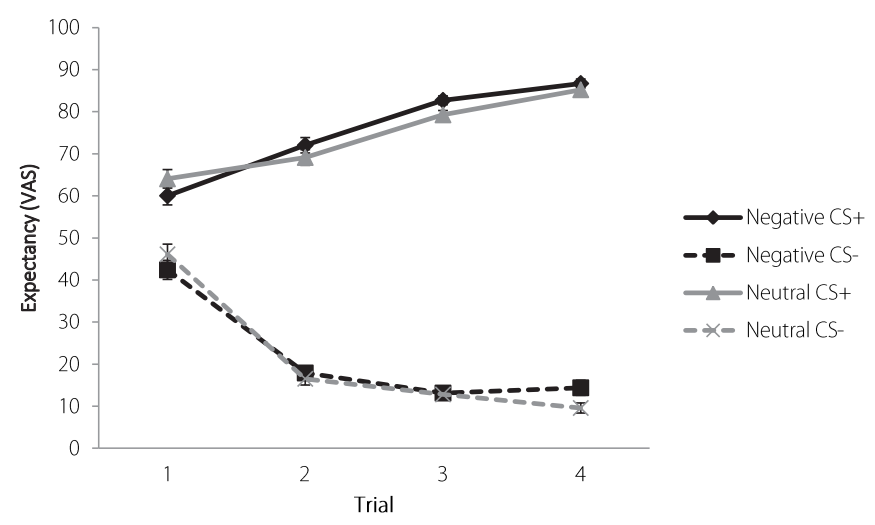

Figure 6.2 Mean US expectancy scores (+ SEM) by CS-type and trial, for the negative and neutral conditions separately 
between the negative and neutral conditions. Impulsivity did again not exert any influence on this interaction, $F(2.06,230.76)=1.27, p=.28$.

\section{US desire}

The ANCOVA on desire to eat chocolate mousse also showed differential acquisition through a significant Condition X CS-type XTrial interaction, $F(2.78,311.49)=4.08, p=.009$, indicating differences between the conditions in the acquisition of desire (see Figure 6.3): participants in the neutral mood condition showed a different pattern of desire than the participants in the negative mood condition. To investigate the differences in acquisition, a CS-Type $X$ Condition interaction was computed for trials 2, 3 and 4 . There was no significant CS-Type $X$ Condition interaction at trial $2, F(1,115)=.24, p=.62$, or trial $3, F(1,115)=2.55, p=.11$, but at trial 4 differential desire to eat was significantly smaller for the negative compared to the neutral condition $F(1,115)=5.48, p=.021$, indicating a less strong conditioning of desire for people in a negative than in a neutral mood. However, at trial 4 participants in the negative condition still reported higher desire in response to the CS+ than the CS-, t(6o) $=3.74, p<.001$. So, successful conditioning of desire to eat was observed in both conditions. Impulsivity did not influence differential acquisition of desire to eat, CS-Type X Trial X Impulsivity, $F(2.78$, $311.49)=.11, p=.95$ and Condition X CS-Type XTrial X Impulsivity, $F(2.78,311.49)=1.77, p=.16$.

Taken together, our conditioning procedure was successful. However, participants in a negative mood did not show facilitated appetitive conditioning as measured by expectancy or desire compared to participants in a neutral mood. In fact, at the end of the

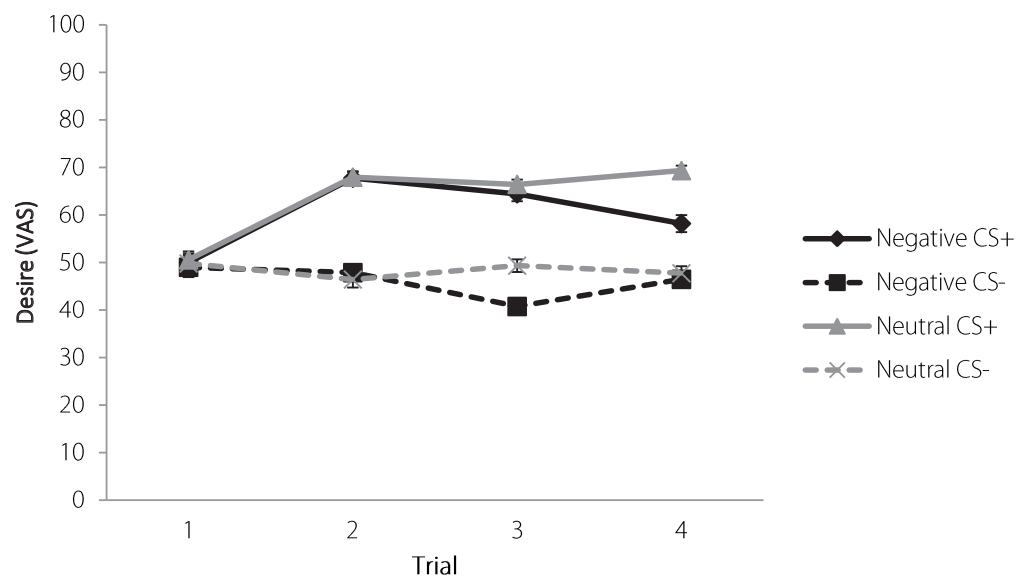

Figure 6.3 Mean US desire scores (+ SEM) by CS-type and trial, for the negative and neutral conditions separately 
conditioning procedure, conditioned desire to eat was significantly lower in the negative mood compared to the neutral mood group. Conditioned expectancy to eat did not differ between groups.

\section{CS liking}

A Condition X CS-type X Time mixed ANCOVA yielded a significant CS-type X Time interaction, $F(1,112)=104.05, p<.001$, indicating a change in differential liking of the CS+ vs. CS- during conditioning. As expected, no differences in CS liking were found between the two mood conditions, as shown by a non-significant three-way interaction, $F(1,112)=1.06, p$ $=.31$. Overall, liking for the CS+ increased slightly and almost reached statistical significance (baseline $M=67.31, S D=18.14$, post $M=70.40, S D=15.18, F(1,116)=3.86, p=.052$ ). Liking for the CS- decreased significantly over time (baseline $M=65.38, S D=17.74$, post $M=43.38$, SD $=19.47, F(1,116)=151.28, p<.001)$. Thus, mostly as a consequence of a significant decrease in liking of the CS-, the CS+ was more liked than the CS- in both mood conditions after conditioning. There was no effect of impulsivity on differential CS liking for either the Time X CS-Type interaction, $F(1,112)=1.62, p=.21$ or the Time X CS-Type X Condition interaction , $F(1,112)=.38, p=.57$. With regard to CS preference, the CS+ was chosen by $79 \%(n=95)(p$ $<$.001) of the participants. These data again show successful conditioning, with a difference in liking of the CS+ and CS- emerging after conditioning, but no significant differences between conditions.

\section{Saliva}

A Condition X CS-Type X Time mixed ANCOVA revealed a main effect of CS-type $F(1,102)=$ $4.04, p=.047$, indicating higher salivation in response to the $C S+(M=.97, S D=.08)$ than the CS- $(M=.74, S D=.08)$. Furthermore, a main time effect for salivation was found, $F(1,102)=$ 27.37, $p<.001$, but no CS-Type X Time interaction, $F(1,102)=2.35, p=.13$, nor a Condition $X$ CS-Type $X$ Time interaction, $F(1,102)=1.53, p=.22$. Impulsivity did not exert any influence on salivation, $F(1,102)=.13, p=.72$. The salivation data is depicted in Figure 6.4. Our hypothesis that participants in a negative mood would produce a stronger conditioned salivation response than participants in a neutral mood when presented with the CS+ could not be confirmed.

\section{Desire to eat before taste test}

An ANCOVA on reported desire to eat when presented with the three bowls of chocolate mousse just before the taste test revealed no significant main effect of Condition, $F(1,104)$ 


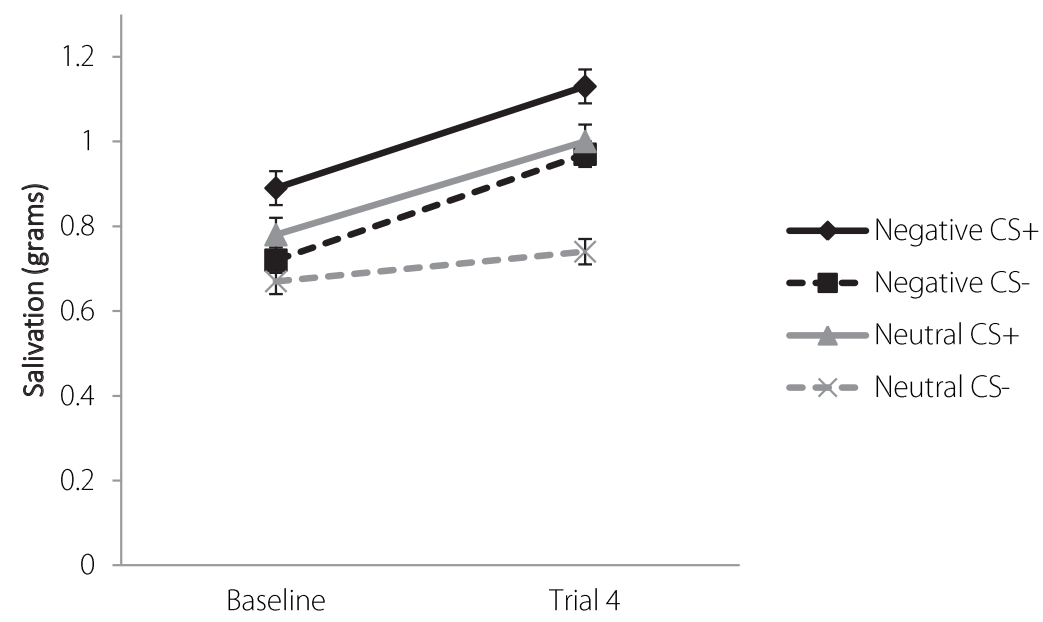

Figure 6.4 Mean salivation in grams at baseline and after the conditioning procedure for all four conditions separately

$=.70, p=.40$. However, there was a significant main effect of CS-type, $F(1,104)=5.43, p=$ .02, as well as a significant Condition X CS-Type interaction, $F(1,104)=4.10, p=.045$. The participants in a negative mood showed more desire to eat when being in the presence of the CS+ compared to the CS-, whereas participants in a neutral mood did not (Negative $C S+: M=75.13, S D=16.41$; Negative $C S-: M=60.00, S D=23.27, F(1,60)=8.56, p=.005 ;$ Neutral $C S+: M=70.33, S D=21.73 ;$ Neutral CS-: $M=69.93, S D=19.56, F(1,58)=.006, p=.94)$. Although there was no Condition X CS-Type interaction with impulsivity, $F(1,104)=1.02, p=.32$, there was a significant interaction with dietary restraint, $F(1,104)=6.16, p=.015$ (adjusted $a=$ .016). A moderated regression analysis showed no difference in desire to eat in presence of the CS+ or CS- between people high and low in restraint when in a neutral mood, CS-Type $X$ Dietary Restraint $\beta=.06, t(58)=.29, p=.78$. However, in a negative mood, high and low restrained eaters did not differ in desire to eat in presence of the CS- , but did so in presence of the CS+, CS-Type X Dietary Restraint $\beta=-.60, t(60)=-2.98, p=.004$. Specifically those low in restraint reported a higher desire to eat when the CS+ was present.

\section{Food intake}

With regard to food intake, there was no main effect of mood condition, $F(1,104)=.002, p=$ .97, and no main effect of CS-Type, $F(1,104)=.05, p=.83$. The Condition X CS-Type interaction was also not significant, $F(1,104)=1.57, p=.21$. These results indicate that participants consumed the same amount of food, irrespective of mood or CS-Type (Negative CS+: M = 
69.94, $S E=6.53 ;$ Negative CS-: $M=60.21, S E=6.40 ;$ Neutral $C S+: M=61.34, S E=6.31 ;$ Neutral CS-: $M=68.13$, SE = 7.10). We did however find a 3-way interaction between Condition, CS-Type, and Impulsivity, $F(1,104)=4.08, p=.046$, suggesting that degree of impulsivity influences food intake after appetitive conditioning in a neutral vs. negative mood.

\section{Impulsivity and food intake}

To follow-up on the significant 3-way interaction, CS-Type $x$ Impulsivity interactions were conducted for the two mood conditions. For the neutral condition, the analysis showed a significant interaction between CS-Type and BIS-11 score, $\beta=.44, t(58)=2.35, p=.023$, indicating that intake in response to the CS+ and CS- is dependent on degree of impulsivity. More specifically, and contrary to expectations, at high levels of impulsivity, chocolate mousse intake was significantly lower when the CS+ was present than when the CS- was present, $\beta=-.46, t(58)=-2.62, p=.011$. At low levels of impulsivity, food intake in response to the CS+ and CS- did not differ, $\beta=.13, t(58)=.70, p=.49$. Furthermore, within the Neutral CS+ group, food intake was significantly lower for high impulsive compared to low impulsive participants, $\beta=-.55, t(58)=-2.83, p=.006$, whereas there was no such difference within the Neutral CS- group, $\beta=.04, t(58)=.22, p=83$.

With regard to the negative condition, no significant effects of CS-Type and impulsivity on mousse intake were found, $\beta=.23, t(60)=1.27, p=.21$. Within the high impulsive and

Table 6.2 Results of moderated regression analysis of condition and BIS-11 scores on chocolate mousse intake

\begin{tabular}{|c|c|c|c|c|c|c|c|c|c|c|c|c|}
\hline \multirow{3}{*}{ Steps and variables ${ }^{\dagger}$} & \multicolumn{6}{|c|}{ Neutral condition $(n=59)$} & \multicolumn{6}{|c|}{ Negative condition $(n=61)$} \\
\hline & \multicolumn{2}{|c|}{ Step 1} & \multicolumn{2}{|c|}{ Step 2} & \multicolumn{2}{|c|}{ Step 3} & \multicolumn{2}{|c|}{ Step 1} & \multicolumn{2}{|c|}{ Step 2} & \multicolumn{2}{|c|}{ Step 3} \\
\hline & B & SE & B & SE & B & SE & B & SE & B & SE & B & SE \\
\hline Emotional Eating & 16.12 & 8.58 & 15.75 & 8.86 & $18.01^{*}$ & 8.56 & 4.31 & 6.72 & 2.54 & 6.89 & .97 & 6.96 \\
\hline Dietary Restraint & -1.86 & 5.98 & -2.76 & 6.05 & -2.55 & 5.81 & $-10.63^{*}$ & 5.02 & -8.72 & 5.18 & -9.85 & 5.23 \\
\hline Condition^ $\wedge$ & & & -13.10 & 9.90 & -12.59 & 9.51 & & & 8.30 & 8.89 & 7.64 & 8.85 \\
\hline BIS-11 & & & -.83 & .53 & .15 & .66 & & & .53 & .44 & .02 & .60 \\
\hline Condition X BIS-11 & & & & & $-2.34^{*}$ & 1.00 & & & & & 1.13 & .89 \\
\hline$R^{2}$ & \multicolumn{2}{|c|}{.060} & \multicolumn{2}{|c|}{.131} & \multicolumn{2}{|c|}{.213} & \multicolumn{2}{|c|}{.075} & \multicolumn{2}{|c|}{.113} & \multicolumn{2}{|c|}{.139} \\
\hline$\Delta R^{2}$ & \multicolumn{2}{|c|}{.060} & \multicolumn{2}{|c|}{.071} & \multicolumn{2}{|c|}{$.082^{*}$} & \multicolumn{2}{|c|}{.075} & \multicolumn{2}{|c|}{.039} & \multicolumn{2}{|c|}{.025} \\
\hline
\end{tabular}

† Note: B is the unstandardized regression coefficient.

$\wedge \mathrm{CS}$ - is reference group

${ }^{*} p<.05$. 

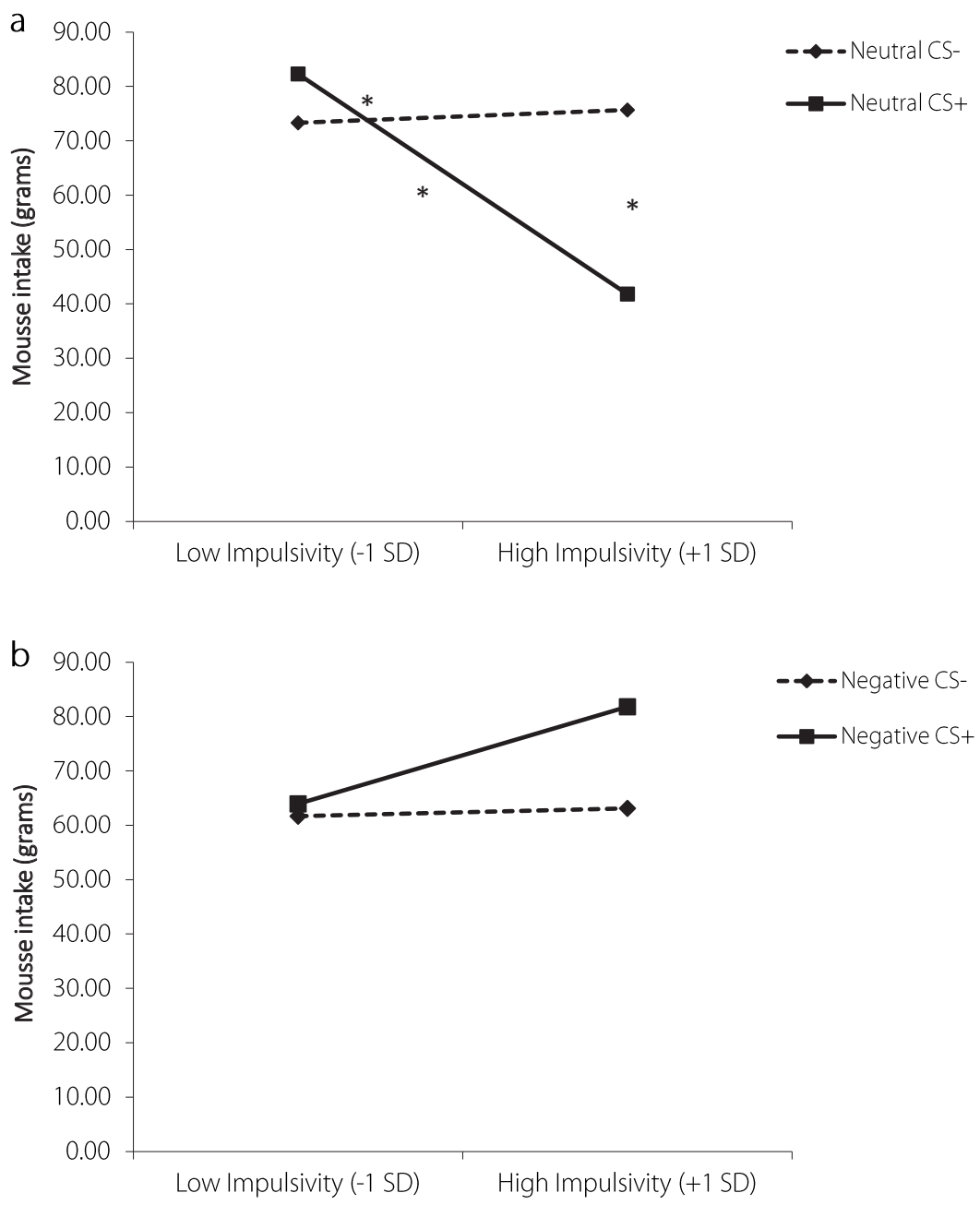

Figure 6.5 Chocolate mousse intake as a function of neutral (a) or negative (b) condition and impulsivity scores (-1 SD and +1 SD from the mean BIS-11 score, respectively). The asterisks indicate the significant effects. 
low impulsive groups there were no differences in intake in response to the CS+ or CS-, and there was no effect of impulsivity on intake within the Negative CS+ and Negative CS- groups, all beta weights, $p>$.13. In short, whereas impulsivity moderated CS-related food intake in the neutral condition, this was not true for the negative condition. Simple slopes and interactions with standardized regression coefficients for participants scoring high (+ 1 SD) and low (-1 SD) on impulsivity are plotted in Figures 6.5 a (neutral condition) and 6.5b (negative condition). Results of both regression analyses can be found in Table 6.2.

\section{DISCUSSION}

In the current study we took a first step in shedding light on the origin of emotional eating by testing whether classical conditioning processes might be involved. More specifically, we investigated whether being in a negative mood facilitates appetitive conditioning, as measured by the expectancy to eat, desire to eat, saliva production and food intake. The results indicate that the conditioning procedure in itself was successful; that is, differential acquisition of eating expectancy was found in both conditions. In other words, participants successfully learned associations between neutral cues and the presence or absence of food. With regard to the effects of this procedure, participants in both conditions also showed differential acquisition of desire to eat. These findings are in line with recent previous studies demonstrating Pavlovian appetitive conditioning in humans (Papachristou et al., 2013; Van den Akker et al., 2013; Van Gucht et al., 2013; Van Gucht et al., 2010; Van Gucht, Vansteenwegen, Beckers, \& Van den Bergh, 2008; Van Gucht, Vansteenwegen, Van den Bergh, \& Beckers, 2008). Contrary to our hypothesis, however, we could not demonstrate facilitated appetitive conditioning in participants in a negative compared to a neutral mood. When it comes to eating expectations, there were no differences in the differential acquisition of learned expectations to eat between the two mood conditions. The desire to eat showed however an interesting difference: Both mood conditions showed conditioned eating desires, but participants in the negative mood condition showed a significantly smaller differential response to the CSs at trial 4, meaning that their learned appetitive desire to eat was weaker than the learned appetitive desire in the neutral mood condition. Whether this is the start of a trend in appetitive conditioning responses or a temporary effect remains to be seen - our design consisted of only 4 trials. However, data on desire to eat immediately before the taste test suggest that a delayed learning effect might take place. Participants in the Negative CS+ condition reported a significantly higher desire to eat on an additional VAS-scale adminis- 
tered directly before the taste test, when the food was presented, compared to participants in the Negative CS- condition. No such difference was found between the Neutral CS+ and CS- groups. This finding suggests the successful learning of a differential response to desire to eat for participants in a negative compared to a neutral mood when presented with a large quantity of food after a conditioning procedure. Another study from our lab (Schyns, Roefs, Mulkens, \& Jansen, submitted for publication) recently found an identical pattern: participants who underwent a food cue exposure training (i.e., prolonged exposure to the sight and smell of a well-liked food without consuming the food, aimed at extinguishing craving for and subsequent intake of that particular food) showed a strong decrease in desire to eat specifically after the exposure procedure, but before the start of a taste test. It could be that a delayed learning effect occurs in certain experimental groups, in which the effects of a conditioning or extinction procedure only become visible after a (short) break. Future studies could look into this by varying the time-interval of a post-conditioning or post-exposure desire measure. As the common factor between the two studies is the presence of a large amount of food and the expectancy to eat, another possibility is that this presence of food and/or expectancy to eat affects conditioned or extinguished desire to eat in the intended direction: increased desire to eat after a conditioning procedure aimed at increasing eating desires, and a decreased desire to eat after an extinction procedure aimed at reducing eating desires. Why such an increase or decrease would occur is a question for future research. Interestingly, in our study, the difference in desire to eat just before the taste test did not translate to a difference in actual food intake: The participants in the neutral and negative mood conditions consumed the same amount of food in the presence of the CS+ and CS-. It could be that exposure to large quantities of real food, which can be considered to be a very strong stimulus, overruled the experimentally conditioned associations, thereby nullifying their effects. Conditioning in real life is likely to be longer and stronger (lasting for months or years), which might be required for conditioned consumption effects.

With regard to salivation, we found no evidence for stronger conditioning in the negative compared to the neutral mood condition. Although salivation increased significantly over time, perhaps due to the exposure to and intake of food during the conditioning procedure, there were no differences in this increase with regard to the CS+ and CS- between the two mood conditions. When looking at the figure depicting salivary responses, it is interesting to note that there is some indication that participants in the negative mood condition displayed increased salivation in response to both the CS+ and CS-, whereas participants in the neutral mood condition showed such a response only when presented with the CS+. Why participants in a negative mood would show increased salivation in response to the 
CS- is unclear, especially considering the conditioned expectations to eat and the learned desire to eat that were both found in the negative mood condition. The inhibited salivary response to the CS- in the neutral mood condition would indicate that they have learned that the CS- would not be followed by food intake. The negative mood condition, on the other hand, did not show evidence of inhibited salivation to the CS-. In anticipation of more research on this topic, it could be that a negative mood generally leads to increased salivation, and that this general response is stronger than a conditioned inhibitory response. The few existing findings on mood states influencing salivation are mixed. Bulik, Lawson, and Carter (1996) reported a negative association between depression levels and salivation, while studies on the relationship between anxiety or stress and salivation have shown either an increase (Rohleder, Wolf, Maldonado, \& Kirschbaum, 2006), a decrease (Bates \& Adams, 1968) or no salivary changes (Ben-Aryeh et al., 1985; Bulik et al., 1996). With our negative mood manipulation we cannot be sure exactly what kind of negative emotions we tapped into and how this might have influenced salivary responding.

Similar to Van den Akker et al. (2013) and Papachristou et al. (2013) we found no effect of impulsivity on expectancy, desire and salivation. However, whereas Van den Akker et al. (2013) found an increase in food intake for high-impulsive participants in response to the CS+ vs. CS-, we found a decrease. Our neutral-mood participants, who are comparable to the participants in the Van den Akker et al. (2013) study, showed lower food intake when presented with the CS+ compared to the CS-. Furthermore, in response to the CS+, the neutral high-impulsive participants consumed less chocolate mousse than the neutral low-impulsive participants. These results are unexpected, as previous studies have generally been consistent in their findings that impulsivity is related to increased food intake (Guerrieri et al., 2008). It might be that the mixed results are due to differences in strength or type of impulsivity not captured by the BIS-11. Whether our findings are spurious or whether impulsivity can indeed inhibit food intake on some occasions is a question for further research.

Interestingly, we did not find any influence of emotional eater status on appetitive conditioning in a negative mood. It could be that our sample of normal-weight healthy students did not contain many, if any, true emotional eaters. Although our participants scored just above average according to the DEBQ norm-scores for female students (our sample $M=2.74, S D=.62$; norm-scores average is 2.61-2.66 (Van Strien, 2005)), previous studies with student and morbidly obese samples were unable to demonstrate emotional eating in these groups (Adriaanse, de Ridder, \& Evers, 2011; Bongers, Jansen, Havermans, Roefs, \& Nederkoorn, 2013; Bongers, Jansen, Houben, \& Roefs, 2013; Brogan \& Hevey, 2013; 
Evers, de Ridder, \& Adriaanse, 2009). These studies raise the question whether the DEBQ is a valid enough measure to identify emotional eaters. For now, there is no evidence that emotional eaters, as selected by the $\mathrm{DEBQ}$, are more susceptible to conditioned eating when emotional than non-emotional eaters. It could be of interest to investigate the influence of emotional eating on appetitive conditioning in a study in clinically identified samples of emotional eaters.

An elaboration on the design of the current study could test the idea that emotions act as an occasion setter in the development of emotional eating. In this case, the negative emotion would function as a CS that signals the occurrence of another CS-US association, and the CR only occurs when the CS-US association is accompanied by the negative emotion. Applied to the current study, this would entail that participants learn that the vase with flowers is followed by chocolate mousse intake only when a negative mood is present, and appetitive responses to the CS only arise when participants are in a negative mood. Another interesting endeavour for future research would be to investigate the first proposed pathway: it is the emotions themselves that become conditioned stimuli and elicit appetitive responses. Previous studies have shown that a variety of initially neutral cues can function as CSs in appetitive conditioning in humans, including objects, colours, and contexts (Birch, McPhee, Sullivan, \& Johnson, 1989; Dols, Willems, Van Den Hout, \& Bittoun, 2000; Thewissen, Havermans, Geschwind, Van den Hout, \& Jansen, 2007; Thewissen, Van Den Hout, Havermans, \& Jansen, 2005; Van den Akker et al., 2013; Van Gucht, Vansteenwegen, Beckers, \& Van den Bergh, 2008; Van Gucht, Vansteenwegen, Van den Bergh, \& Beckers, 2008). It seems plausible that emotions are no exception.

The present study benefits from several strengths, such as a manipulation check to ensure both a successful mood induction and successful conditioning (expectancy ratings), as well as the use of subjective, physiological and behavioural measures of conditioned responding. However, some limitations should also be noted. First, considering that our sample consisted of healthy female undergraduate students, we cannot generalize these results to other groups of interest, such as males or people with overweight or obesity. Second, although our sadness induction was very effective, we cannot be sure if any additional emotions were elicited in our participants (e.g., anger, nostalgia, remorse, anxiety). This seems to be an insurmountable issue however, as it would be very hard, if not impossible, to elicit one single, pure emotion in humans. If additional emotions were elicited, this could have influenced the subjective, physiological or behavioural measures. A third limitation concerns the stimuli we used. When participants were presented with the CS-, this was followed by an empty spoon. We chose this empty spoon to resemble the CS+ 
trials (in which a spoon with chocolate mousse was presented) as closely as possible, while still making clear to participants that no eating would occur. However, it is possible that the spoon was not neutral enough, given its typical association with eating, which caused participants to still create a link between the CS- vase and food. Although this did not influence conditioned expectancies and desires to eat, it could have influenced the null findings with regard to actual food intake. Fourth, although widely used in eating research with student samples, the BIS-11 has not been validated in this population.

To conclude, we are the first to demonstrate successful appetitive conditioning in neutral and negative moods. However, a negative mood does not seem to facilitate this conditioning process.

\section{ACKNOWLEDGEMENTS}

This study is part of an ongoing project that is financed by the Netherlands Organisation for Scientific Research (NWO): Vici Grant 453.10.006, awarded to Anita Jansen. 

7

Emotional eating and Pavlovian learning:

Evidence for conditioned appetitive

responding to negative emotional states

Bongers, P. \& Jansen, A. (in press). Emotional eating and Pavlovian learning: Evidence for conditioned appetitive responding to negative emotional states. Cognition and Emotion. 


\section{ABSTRACT}

Appetitive learning has been demonstrated several times using neutral cues or contexts as a predictor of food intake and it has been shown that humans easily learn cued desires for foods. It has however never been studied whether internal cues are also capable of appetitive conditioning. In this study, we tested whether humans can learn cued eating desires to negative moods as conditioned stimuli, thereby offering a potential explanation of emotional eating. Female participants were randomly presented with ten different stimuli eliciting either negative or neutral emotional states, with one of these states paired with eating chocolate. Expectancy to eat, desire to eat, salivation and unpleasantness of experiencing negative emotions were assessed. After conditioning, participants were brought into a negative emotional state and were asked to choose between money and chocolate. Data showed differential conditioned responding on the expectancy and desire measures, but not on salivation. Specific conditioned effects were obtained for participants with a higher BMI on the choice task, and for participants high on emotional eating on the unpleasantness ratings. These findings provide the first experimental evidence for the idea that negative emotions can act as conditioned stimuli, and might suggest that classical conditioning is involved in emotional eating.

Keywords: Appetitive conditioning; Emotional eating; Emotional states; Mood; Cue reactivity. 


\section{INTRODUCTION}

Palatable high-calorie foods have high potential for conditioning. Every time one eats, there is an opportunity to associate the eating with cues or contexts that are present at the time (Bouton, 2011; Bouton, Woods, Moody, Sunsay, \& García-Gutiérrez, 2006). The number of daily possibilities to associate cues and contexts with palatable food intake is almost endless (Bouton, 2011). The place where one eats, food preparing rituals, food smells and tastes, time of the day; they are all potential signals for eating and classical conditioning. Animal studies show that physiological responses to food intake (e.g. insulin-release, blood-sugar increase and salivation) can be elicited by any stimulus predictive of eating (Bouton, et al., 2006; Jansen, Havermans, \& Nederkoorn, 2011a). Context-cues associated with palatable food intake drive overeating in rats, even when they are sated and when the food is less preferred (Boggiano, Dorsey, Thomas, \& Murdaugh, 2009; Weingarten, 1983).

In humans, it likewise appears to be relatively easy to learn cued eating desires and cued overeating through classical conditioning. In a series of experimental studies, successful appetitive conditioning to neutral cues like serving trays, vases and children's jewellery boxes was demonstrated by systematically pairing these neutral cues with the eating of something palatable, like a small piece of chocolate (Bongers, van den Akker, Havermans, \& Jansen, 2015; Papachristou, Nederkoorn, Beunen, \& Jansen, 2013; van den Akker, Havermans, Bouton, \& Jansen, 2014; van den Akker, Havermans, \& Jansen, 2015; Van Gucht, Baeyens, Hermans, \& Beckers, 2013; Van Gucht, Baeyens, Vansteenwegen, Hermans, \& Beckers, 2010; Van Gucht, Vansteenwegen, Beckers, \& Van Den Bergh, 2008a; Van Gucht, Vansteenwegen, Van den Bergh, \& Beckers, 2008b). These studies consistently show that after the learning of a stimulus predicting intake, the mere presence of the food-predictive stimulus is sufficient to elicit eating expectations and eating desires. Also when satiated a signal that predicts consumption is able to elicit food desires; just thinking of how delicious the dessert would taste might make people feel 'hungry' and eat, even when they had a large meal. Such cued eating desires increase the risk of overeating (Ferriday \& Brunstrom, 2011; Jansen, 1998; Jansen et al., 2011a). A mere four cue - intake trials being enough to obtain these effects shows that appetitive learning develops quickly. Appetitive conditioning was also demonstrated with contexts instead of cues. Birch, McPhee, Sullivan, and Johnson (1989) showed contextual conditioning of meal initiation, with pre-school children showing significantly shorter latencies to start eating in an environment previously paired with eating compared to an environment not associated with eating. Van den Akker, Jansen, Frentz, and Havermans (2013) conducted a virtual reality study in which distinct physical environments (contexts) 
were paired with food intake. As with all kinds of other external cues, contexts easily developed into conditioned signals eliciting increased food expectancies, food craving, salivation and intake. Though these data show that human appetitive conditioning to external cues and contexts is a quite robust finding, it has never been studied whether internal cues are also capable of appetitive conditioning. Of special interest is whether specific emotional states have the potential to act as conditioned stimuli for overeating. If emotional states are systematically associated with eating they might, in the end, easily trigger eating desires and overeating. Overeating induced by emotions is the hallmark of emotional eating, a term first coined in the 1960 s (Bruch, 1964). Although originally introduced as an explanation for obesity and with a focus on merely negative emotions, emotional eating has since evolved into a behaviour also ascribed to other populations (Herman \& Mack, 1975; Nisbett, 1968; Van Strien, Frijters, Bergers, \& Defares, 1986). In addition, studies have shown that positive emotions are also capable of eliciting overeating in emotional eaters (Bongers, Jansen, Havermans, Roefs, \& Nederkoorn, 2013a; Bongers, Jansen, Houben, \& Roefs, 2013b; Evers, Adriaanse, de Ridder, \& de Witt Huberts, 2013).

If emotional eating is a specific demonstration of appetitive conditioning, two possible pathways through which this could occur are most obvious (Bongers et al., 2015). The first pathway proposes that emotional eating develops through the facilitating effect a negative mood has on the learning of an association between a neutral cue and food intake. This hypothesis was based on the idea that the eating of highly palatable foods is rewarding and mood-enhancing (see for example Coletta et al., 2009; Macht \& Dettmer, 2006; Macht \& Mueller, 2007; Small, Zatorre, Dagher, Evans, \& Jones-Gotman, 2001), which is especially reinforcing for individuals who are in a negative mood. They would benefit from learning that certain cues predict tasty foods, and thus an alleviation of a negative mood. To test this, half of our participants underwent a differential conditioning procedure while in a negative mood, the other half while in a neutral mood. One distinct vase with flowers was repeatedly paired with food intake, whereas a second distinct vase was not. Expectancy to eat, desire to eat, salivation and food intake were measured. Though appetitive conditioning was demonstrated, no clear evidence for a facilitating effect of negative mood on appetitive conditioning was found (Bongers et al., 2015). The second pathway, which is under investigation in the current study, specifies the emotions in itself as conditioned stimuli. If a particular emotional state is (nearly) always followed by the intake of palatable high-calorie foods, the specific emotional state will become a predictor signaling high calorie food intake. It then follows that the mere experience of such an emotion will automatically elicit food cravings, a salivation response and ultimately cued consumption. Although never experimentally 
tested, emotions have been suggested to have the potential to grow into conditioned stimuli (Greeno \& Wing, 1994; Jansen, 1998; Jansen et al., 2011a; Macht, 2008; Wardle, 1990).

In the present study we investigate whether, after repeatedly being paired with chocolate (US, or unconditioned stimulus), negative emotional states can act as conditioned appetitive stimuli (CSs) that elicit cue reactivity, like cued eating expectations, cued eating desires, and cued salivation (conditioned responses; CRs). We included expectancy to eat and desire to eat ratings as self-report measures, saliva production as a physiological measure, and a money vs. chocolate choice task during negative mood as a behavioural measure. Because the eating of high calorie foods is especially rewarding in response to negative emotional states, we expect the appetitive conditioning of negative emotional mood cues to be stronger, quicker and easier than the appetitive conditioning of neutral mood cues. We hypothesize differential responding to the CSs (i.e., negative and neutral emotional states) on the self-report and physiological measures. With regard to the behavioural measure, we expect participants conditioned to eat in a negative emotional state to have a stronger preference for chocolate than participants conditioned to eat in a neutral state. Finally, some earlier appetitive conditioning studies show a significant increase in CS+ (i.e., the CS paired with food) liking after conditioning (Bongers et al., 2015; Papachristou et al., 2013; van den Akker et al., 2014; van den Akker et al., 2015; Van den Akker et al., 2013; Van Gucht et al., 2010). If appetitive conditioning with negative emotions as the CS+ is successful, and CS+ liking occurs, negative emotions should be evaluated as less negative after conditioning. We therefore predicted that the experienced unpleasantness of negative emotions will reduce in individuals for whom negative emotions were paired with chocolate.

\section{METHOD}

\section{Participants}

47 female undergraduate students aged 18 to 30 years old $(M=20.25, S D=2.53)$ participated in the study in return for course credit or a gift voucher worth $€ 12.50$. Sample size was based on previous studies on appetitive conditioning in humans that utilized a similar paradigm (Meyer, Risbrough, Liang, \& Boutelle, 2015; van den Akker et al., 2014; Van Gucht 
et al., 2010; Van Gucht et al., 2008a; Van Gucht et al., 2008b). Participants were recruited through advertisements which referred to a study on 'the influence of concentration and emotions on taste perception. To be eligible for participation, participants had to be female undergraduate students between the ages of 18 and 30, with no food allergies and a liking of chocolate. A single question via email after sign-up assessed chocolate liking on a scale of 1 (does not like chocolate at all) to 5 (likes chocolate very much), and a minimum score of 3 was required. The study was approved by the ethics committee of the Faculty of Psychology and Neuroscience, Maastricht University.

\section{Study design and conditioning procedure}

Participants were randomly divided over two conditions: in the FoodNeg condition ( $\mathrm{n}=$ 22) participants were conditioned to eat during negative emotional states (i.e., receiving chocolate when experiencing a negative emotional state (CS+) and no chocolate in a neutral emotional state (CS-)), in the other (FoodNeu condition, $n=25$ ) this was reversed (i.e., receiving chocolate in a neutral emotional state $(\mathrm{CS}+)$ and no chocolate in a negative emotional state (CS-)). Ten conditioning trials ( 5 trials with negative mood and 5 trials with neutral mood) were randomly presented, with the restriction that the first two and last two trials consisted of a CS+ and CS- trial, counterbalanced across participants. In addition, there could be no more than two consecutive trials of the same type. After each trial there was a 3-minute intertrial interval to return the participants' mood back to its original level.

\section{Stimuli}

\section{CSs}

Five different stimuli were used to evoke negative or neutral emotional states. All stimuli had a negative and a neutral variety and were presented for 3 minutes. All stimuli were pilot tested and found to have the desired effect.

Text. The negative text consisted of a 12 year old girl detailing what happened to her and her family during the Cambodian genocide in the late 1970s. The neutral text was a travel blog of a Dutch girl who visited some well-known Cambodian temples. Both texts consisted of approximately 600 words. Three relevant pictures were added to each text. 
Music. For the negative music, participants listened to a song which elicited sad feelings in them. The song of choice was personal and therefore different for each participant. The neutral music was La Traviata (Prelude to Act 1) by Giuseppe Verdi and was the same for each participant. La Traviata was found to be neutral in a previous study (Mitterschiffthaler, Fu, Dalton, Andrew, \& Williams, 2007).

Film. A scene from the movie The Champ in which a boy watches his father die was used as the negative stimulus. The neutral film explained the making of model train cars.

Memory. To induce a negative mood, participants read and thought about a negative memory. For the neutral mood, participants read and thought about a neutral memory.

Pictures. 18 negative pictures selected from the International Affective Picture System ' (IAPS; Lang, Bradley, \& Cuthbert, 1999) were used to induce a negative mood; those selected for this study were previously rated high on sadness and low on disgust (Mikels, et al., 2005). Each picture was presented for 10s, while Adagio in Sol Minore by Tomaso Albinono played in the background. Adagio in Sol Minore has been found to be an effective inducer of negative mood (Mitterschiffthaler, et al., 2007). For the neutral version, 18 neutral IAPS pictures ${ }^{1}$ were presented for 10 s with Georg Friedrich Haendel's Watermusic Minuet playing in the background. Both the pictures (Lang, et al., 1999) and the music (Mitterschiffthaler, et al., 2007) were reported as neutral in previous studies.

\section{USS}

Small star-shaped chocolates (brand: Maison Blanche Dael) were used as USs. The chocolates were individually presented in a small transparent cup. Participants had a choice between milk and dark chocolate and each chocolate weighed approximately 1.7 grams.

\section{Puzzles}

Participants were instructed to work on puzzles during the 3-minute intertrial interval. It was stressed to participants that although they should focus only on the puzzles, there was no goal to reach and they could switch between puzzles whenever they wanted. A

1 IAPS pictures used for the negative version were 2053, 2141, 2205, 2800, 2900, 3230, 3350, 6570, 6838, 9000, $9041,9050,9415,9421,9520,9530,9611,9910$. IAPS pictures used for the neutral version were 1670, 2191, 2393, $2410,2487,2514,2518,5390,5395,5520,7002,7004,7039,7041,7090,7150,8211,8311$. 
selection of seven different puzzles (e.g., word finder, Sudoku, crossword, connect-the-dots) was available for participants to choose from. The puzzles were used to keep participants occupied during the intertrial interval and to counteract the possibly ongoing effects of the prior (negative) manipulation. A pilot study with ten participants showed this to be an effective technique.

\section{Measures}

Mood. To take into account the variety of negative emotions that could be induced by our stimuli, and because we were interested in the experience of a negative emotional state in general, and not specific negative feelings, participants received the following instructions before the first mood measurement at baseline: "During the study you will experience several negative feelings. These can for example be sadness, anger or anxiety, but also other unpleasant or disagreeable feelings. We will not ask about what kind of feelings you experience specifically, but we do want to know how strong these feelings are." Mood was then rated on a 10omm VAS which asked 'How strong are your negative feelings at this moment?', ranging from 'Not strong at all' to 'Extremely strong'. The VAS scale was presented before and after every stimulus presentation.

Expectancy and desire. Expectancy to receive chocolate and desire to eat chocolate were measured by means of VAS scales after every stimulus presentation. For expectancy, the question was formulated as 'Focus on how you feel right now. How strongly do you expect to receive chocolate at this moment?' and the VAS ranged from 'Not at all' to 'Very much'. The question for desire read 'Focus on how you feel right now. How much do you desire to eat chocolate at this moment?' and the anchor points on the VAS were identical to those of the expectancy-question.

Emotional Valence /CS-liking. At baseline and after conditioning, participants rated on a VAS scale how unpleasant they found it to experience negative emotions. The VAS ranged from 'not unpleasant at all' to 'very unpleasant'.

Imagination. To check whether participants followed instructions and did their best to imagine themselves in the scenes depicted in the stimuli, they were asked how hard they tried to imagine themselves in all situations and how well they managed to do this imagination. Both questions were answered on a VAS ranging from 'not hard at all / not well at all' to 'very hard / very well'. 
Contingency Awareness. Contingency awareness was measured through two questions, framed in the following way: 'You just finished a computer task where you had to empathize with several stimuli. During the task, did you experience a certain feeling or certain feelings which were always followed by eating chocolate?' Answering options were 'yes', 'no', or 'I don't know'. If participants answered 'yes', they were asked to write down which feelings were always followed by chocolate. An identical question was asked where 'feeling(s)' was replaced by 'stimulus/stimuli'.

Salivation. Salivation was measured at baseline and after trials 9 and 10. Dental cotton roles (Hartmann Celluron no. 2) were used to assess saliva production. For one minute, participants placed one cotton role in each side of their mouth, between the cheek and lower gum. They placed an additional half role under their tongue. Cotton roles were measured in grams before and after the salivation measurement on a .01 gram precision kitchen scale.

Choice task. A wooden tray on which a 2-euro coin and a small bag of the star-shaped chocolates (approximately 90 grams, retail price $€ 2$ ) were presented was put in front of the participants and they were invited to take the item they would most like to have at that moment.

$D E B Q$. The Emotional Eating (EE) and Dietary Restraint (DR) subscales Dutch Eating Behaviour Questionnaire (DEBQ; Van Strien, 2005) were used to assess emotional eating and dietary restraint. The scales consist of 13 and 10 questions, respectively, and items are answered on a 5-point Likert scale. Higher scores indicate a higher degree of emotional or restrained eating behaviour.

Compliance with eating instructions. Participants were asked to indicate when and what they had last eaten before participating in the experiment. Additionally, they were asked whether they had consumed chocolate in the last 24 hours, and if so, when.

Height and weight. Participants' height and weight was measured while wearing street clothes but no shoes. 


\section{Procedure}

After sign-up, participants received an email with instructions to not consume any chocolate in the 24 hours before the experiment, as well as to eat something small (e.g., an apple or sandwich) 2 hours before participation, but to refrain from eating or drinking (except water) thereafter. They were also asked to send the artists and titles of two songs that made them happy and two songs that made them sad to the experimenter. Finally, they were asked to write down a personal sad memory, a personal happy memory, and a neutral memory (i.e., a description of the route from home to the university library) on three separate sheets of paper and to take those with them to the lab on the testing day. The happy music and happy memory were not used in the experiment, but were included to keep the content of the study obscure. Upon arrival in the lab, participants were asked to put cotton rolls in their mouth for the baseline saliva measurement. They then answered the baseline question for CS liking, and following this received instructions (orally and in writing) with regard to the conditioning part of the experiment: "Next you will see and hear a number of different stimuli. With stimuli we mean film clips, music, memories, texts and pictures. The film clips, music and pictures will be shown on the screen, the memories and texts are on paper. Some of these stimuli will elicit negative feelings, others will not. After one of the two (so after negative or neutral feelings) you will receive something to eat, after the other you will not. So, after every stimulus, you can predict whether or not you will receive something to eat." The next screen contained the explanation of the mood measurements, and subsequently the first trial started with a mood VAS. Before presentation of every stimulus, the following information appeared on the participants' screen: "You are about to see a stimulus. We want you to fully focus on this stimulus and concentrate on the feelings the stimulus elicits in you. You should really experience the feelings." After 3 minutes stimulus presentation was stopped, and the mood VAS was administered again, followed by the expectancy and desire measures. The experimenter then approached the participant with either an empty cup or a cup with a chocolate in it, dependent on the trial type. Participants were instructed to eat (or not) the chocolate, and following this, the computer screen showed the puzzle instructions. This trial procedure was repeated 9 more times. During trial 9 and 10, the expectancy and desire ratings were followed by a second and third saliva measurement. After trial 10 the participant answered the second VAS to measure CS-liking. Finally, all participants listened to their second sad song. After 3 minutes the music stopped and the experimenter approached the participant and told her that she would get the standard reward for participating, but that the research team would like to give her something extra as a token of appreciation because of the considerable length 


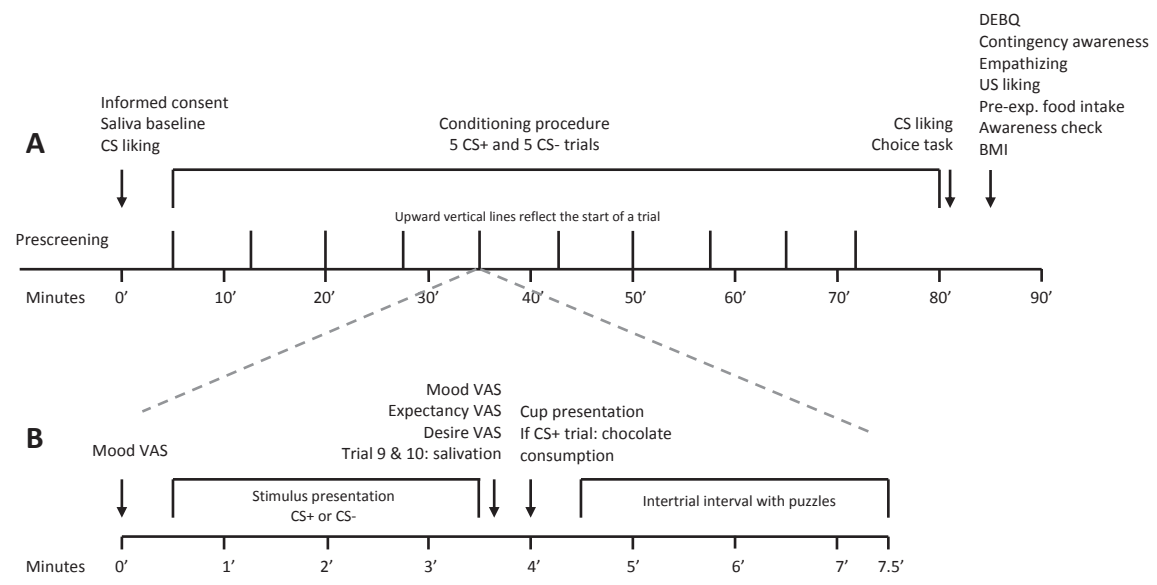

Figure 7.1 Timelines of the full experimental procedure (A) and a single conditioning trial (B).

and negative stimuli in the experiment. After the participants made their choice of either chocolate or money, they were asked to fill out a few more questionnaires, including the $D E B Q$, and the questions regarding contingency awareness, empathizing, US liking, and compliance with eating instructions. They were measured and weighed, received their reward, and thanked for participation. The total duration of the experiment was 90 minutes. Timelines of both the whole experimental procedure and one single conditioning trial are provided in Figure $7.1 \mathrm{a}$ and $7.1 \mathrm{~b}$, respectively. Participants received a debriefing via email after all data collection was completed.

\section{Statistical analyses}

31 participants (18 in the FoodNeg condition) reported contingency awareness on the self-report measure, whereas 16 participants did not or were unclear in their answers (6 in the FoodNeg condition). Closer inspection of expectancy graphs of these 16 participants revealed that 9 of the 16 did show conditioned expectancies in their responses. Analyses were run with and without these 9 participants and revealed a similar pattern of results, and were therefore included in the analysis. The 7 participants who did not report contingency awareness, neither in self-report nor in the expectancy graphs, were all in the FoodNeu condition and were not included in the analyses. The final sample consisted of 40 participants (22 in the FoodNeg condition, 18 in the FoodNeu condition).

A one-way ANOVA was carried out to check for pre-existing baseline differences between the two conditions. To investigate the effectiveness of our mood stimuli, we conducted a 2 
(time: pre vs. post) X 2 (stimulus: negative vs. neutral) Repeated Measures ANOVA for each of the five stimuli. In case of a significant Time $X$ Stimulus interaction, paired sample t-tests were carried out to examine simple effects.

In all analyses on our main outcome variables (expectancy, desire, salivation, CS liking and the choice task), centered BMI and DEBQ EE scores were included as covariates. Because Pearson's correlations revealed a moderate correlation between BMI and DEBQ-DR, $r=.38$, $p=.017$, only BMI was included as a covariate. Given that there were no a priori hypotheses regarding the covariates, a were adjusted to .008 (for expectancy and desire analyses), .016 (for salivation analysis) or .025 (for CS liking analysis) to correct for the number of factor and covariate interactions tested among these variables. Acquisition of expectancy and desire to eat was analyzed in a mixed 2 (Condition: FoodNeg vs. FoodNeu) X 2 (CS-type: CS+ vs. CS-) $X_{5}$ (Trial: $\left.1,2,3,4,5\right)$ ANOVA. Salivary responding was analyzed in a mixed 2 (Condition: FoodNeg vs. FoodNeu) X 2 (CS-type: CS+ vs. CS-) X 3 (Time: baseline, CS+, CS-) ANOVA. A 2 (Condition: FoodNeg vs. FoodNeu) X 2 (Time: pre-conditioning vs. post-conditioning) mixed ANOVA was used to assess changes in CS liking. Additional analyses were performed in the case of significant interactions. When sphericity was violated, Greenhouse-Geisser corrections are reported. A logistic regression (method = Enter) with choice (chocolate vs. money) as the dependent variable, condition (FoodNeg vs. FoodNeu) as the main predictor variable (Block 1), BMI and DEBQ EE scores (Block 2) and their interactions with Condition (Block 3) as covariates was conducted to investigate the effect of condition on reward choice. Reported effect sizes are eta-squared.

\section{RESULTS}

\section{Participant characteristics}

As shown in Table 1, participants did not differ on age, imagination, emotional valence, DEBQ EE, or minutes since last eaten. There were significant condition differences on BMI (Cohen's $d=.75$ ) and DEBQ-DR (Cohen's $d=.49$ ). None of the participants were aware of the hypotheses of the study.

\section{Emotional states manipulation}

Pre- and post mood scores per stimulus are displayed in Table 7.2. The Time X Stimulus interactions were significant for all five mood stimuli (all $F_{S}>128.41$, all $p s<.000$ ). Follow-up paired-sample t-tests to test for simple effects revealed a significant increase in negative 
Table 7.1 Means and standard deviations of participant characteristics per condition

\begin{tabular}{lllllll}
\hline & $\begin{array}{l}\text { FoodNeg } \\
(\mathrm{n}=22)\end{array}$ & & $\begin{array}{l}\text { FoodNeu } \\
(\mathrm{n}=18)\end{array}$ & & $F$ & $P$ \\
\hline Age & $M$ & SD & $M$ & SD & & \\
BMl & 19.86 & 2.64 & 20.72 & 2.37 & 1.15 & .29 \\
Imagination (effort) & 21.59 & 1.97 & 24.94 & 5.97 & 6.15 & .02 \\
Imagination (success) & 80.59 & 9.26 & 84.50 & 8.63 & 1.88 & .18 \\
Emotional valence (baseline) & 75.00 & 10.07 & 77.56 & 13.40 & .47 & .50 \\
DEBQ-EE' & 64.41 & 21.12 & 68.06 & 22.91 & .27 & .60 \\
DEBQ-DR' & 3.01 & .53 & 2.72 & .64 & 2.57 & .12 \\
Minutes since last eaten & 2.62 & .66 & 3.05 & .54 & 4.87 & .03 \\
\hline
\end{tabular}

${ }^{1}$ DEBQ-EE: emotional eating scale of the DEBQ; DEBQ-DR: dietary restraint scale of the DEBQ

Table 7.2 Means and standard deviations of pre and post mood score (on a scale from 0 to 100 with higher scores indicating higher negative feelings) per stimulus type

\begin{tabular}{|c|c|c|c|c|c|c|c|}
\hline & \multicolumn{2}{|c|}{$\operatorname{Pre}(n=40)$} & \multicolumn{2}{|c|}{ Post $(n=40)$} & \multirow[t]{2}{*}{$t$} & \multirow[t]{2}{*}{$p$} & \multirow[t]{2}{*}{ Cohen's d } \\
\hline & M & SD & M & SD & & & \\
\hline \multicolumn{8}{|l|}{ Film } \\
\hline Negative & 8.88 & 10.40 & 68.55 & 20.39 & 16.71 & $<.001$ & 2.64 \\
\hline Neutral & 18.43 & 16.90 & 10.50 & 13.42 & 4.34 & $<.001$ & .69 \\
\hline \multicolumn{8}{|l|}{ Memory } \\
\hline Negative & 12.30 & 14.45 & 68.78 & 18.09 & 16.65 & $<.001$ & 2.63 \\
\hline Neutral & 18.58 & 18.44 & 11.68 & 14.69 & 3.04 & .004 & .48 \\
\hline \multicolumn{8}{|l|}{ Pictures } \\
\hline Negative & 12.78 & 14.81 & 61.03 & 20.13 & 14.21 & $<.001$ & 2.25 \\
\hline Neutral & 15.28 & 15.86 & 12.05 & 13.43 & 1.30 & .203 & - \\
\hline \multicolumn{8}{|l|}{ Text } \\
\hline Negative & 11.60 & 14.25 & 63.73 & 19.28 & 13.35 & $<.001$ & 2.11 \\
\hline Neutral & 18.85 & 16.63 & 9.70 & 13.96 & 4.12 & $<.001$ & .66 \\
\hline \multicolumn{8}{|l|}{ Music } \\
\hline Negative & 12.08 & 14.10 & 65.20 & 21.06 & 14.07 & $<.001$ & 2.22 \\
\hline Neutral & 15.88 & 16.22 & 20.53 & 18.98 & 1.24 & .118 & - \\
\hline \multicolumn{8}{|l|}{ Music $\|^{1}$} \\
\hline Negative & 19.90 & 18.31 & 63.60 & 20.76 & 13.04 & $<.001$ & 2.06 \\
\hline
\end{tabular}

\footnotetext{
${ }^{1}$ The second music manipulation, at the end of the conditioning procedure, consisted of a negative version only.
} 

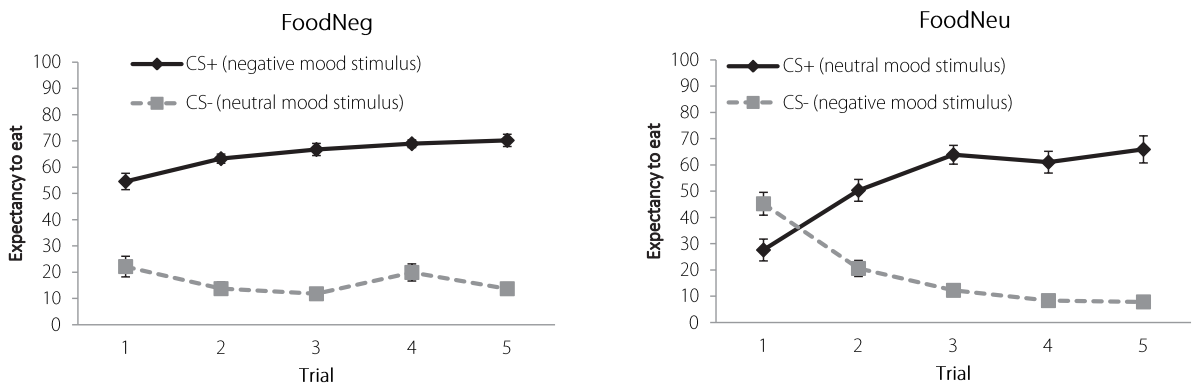

Figure 7.2 Mean US expectancy scores (+SEM) by CS-type and trial, for the FoodNeg and FoodNeu conditions separately

feelings for all five negative stimuli. With regard to the neutral stimuli, there was a slight but significant decrease in negative feelings for the film, memory, and text manipulations. Together, these results show successful mood manipulations.

\section{Conditioning effects}

\section{US Expectancy}

US Expectancy ratings for participants in both conditions are shown in Figure 7.2. A significant Trial X CS-Type X Condition interaction, F $(3.39,115.38)=7.84, p<.001, \eta^{2}=.11$, indicated differences between the FoodNeu and FoodNeg group in expectancy learning. There was no effect of BMI or Emotional Eating (all Fs $<1.51$, all ps $>$.20. Follow-up analyses of Trial XCSType interactions per condition revealed that acquisition was successful in both conditions (Negative: $F(2.42,46.02)=3.47, p=.031, \eta^{2}=.14$; Neutral: $\left.F(4,60)=25.40, p<.001, \eta^{2}=.59\right)$. Closer inspection of expectancy scores on Trial 1 showed a significant CS-Type X Condition interaction on this trial, $F(1,34)=20.37, p<.001, \eta^{2}=.33$, indicating a larger baseline difference between CS+ and CS- for the FoodNeg compared to the FoodNeu condition, although baseline differences on Trial 1 were present in both conditions (Negative: CS+M $=57.73, S D=21.06 ; C S-M=25.45, S D=24.12, F(1,21)=23.37, p<.001, \eta^{2}=.53 ;$ Neutral: $C S+$ $\left.M=31.28, S D=27.05 ; C S-M=45.83, S D=23.50, F(1,17)=4.94, p=.04, \eta^{2}=.23\right)$. There was a significant differentiation between CS+ and CS- trials on Trial $5, F(1,34)=115.09, p<.001, \eta^{2}$ $=.76$, with no differences between conditions, $F(1,34)=.02, p=.88$. 
FoodNeg

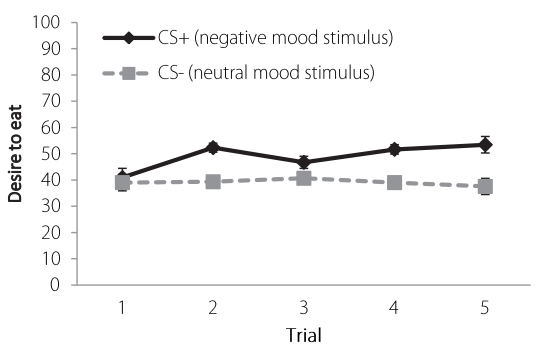

FoodNeu

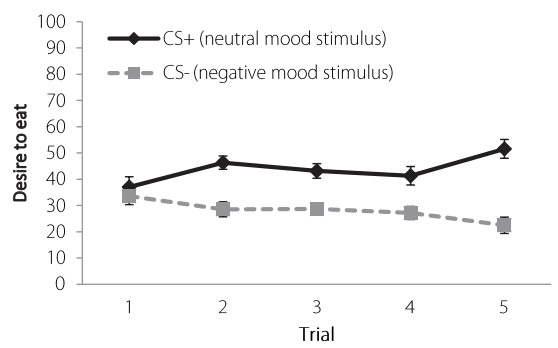

Figure 7.3 Mean US desire scores (+SEM) by CS-type and trial, for the FoodNeg and FoodNeu conditions separately

\section{Desire to eat}

Figure 7.3 displays desire ratings in both conditions. The participants showed a conditioned desire to eat, $F(3.2,108.85)=5.1, p=.002, \eta^{2}=.09$, which did not differ between the two conditions, $F(3.2,108.85)=.60, p=.63$. BMl and Emotional eating did not have a significant effect (all Fs $<3.67$, all ps <.013). Differential desire to eat was present on Trial $5, F(1,34)=$ $13.64, p=.001, \eta^{2}=.27$, with no differences between conditions, $F(1,34)=1.17, p=.29$.

\section{Salivation}

Salivation data of one participant in the neutral condition was missing. There was a main effect of time, $F(1.70,56.00)=3.72, p=.037, \eta^{2}=.09$, indicating a difference in salivation across the three measurements (Negative: baseline $M=.80, S D=.38 ; C S+M=1.18, S D=.82$; $C S-M=1.26, S D=.76 ;$ Neutral: baseline $M=.88, S D=.58 ; C S+M=1.19, S D=.64 ; C S-M=1.03$, $S D=.55$. Pairwise comparisons showed an increase of salivation from baseline to CS+ $(p=$ .04), from baseline to CS- ( $p=.01)$, but no differences in salivation between CS+ and CS- ( $p$ $=.75)$. BMI and EE did not exert any influence, all $F s<1.27$, all $p s>.29$.

\section{Choice task}

In the FoodNeg condition, 19 out of 22 (86\%) participants chose chocolate over money. In the FoodNeu condition, this was 10 out of 18 participants $(56 \%), X^{2}(1)=4.71, p=.03, \mathrm{OR}=$ 5.07. However, after controlling for $\mathrm{BMI}$ and $\mathrm{EE}$ in a logistic regression, the predictive effect of condition was no longer significant. EE X Condition ( $B=1.09, S E=1.81, p=.55)$ and the main effect of $E E(B=1.42, S E=.87, p=.10)$ were not significant and removed from the model. The final model with BMI and BMI X Condition as covariates showed a significant contribution of the interaction to the model $\left(B\right.$ lock $X^{2}(1)=3.96, p=.047$; Model $X^{2}(3)=11.98, p=.007$, 


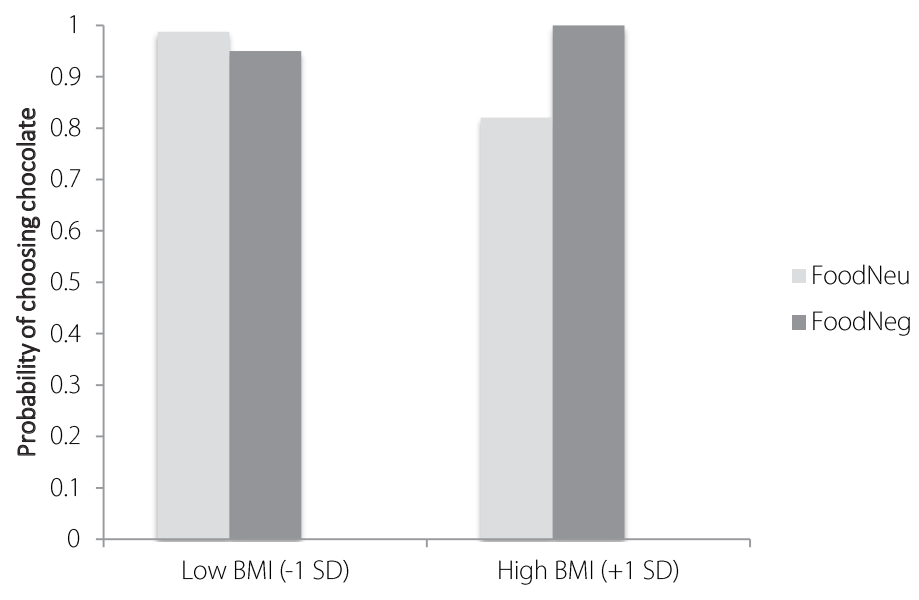

Figure 7.4 Probability of choosing chocolate as a function of condition and BMI (-1SD and +1 SD from the mean BMI score)

Nagelkerke $\mathrm{R}^{2}=.37 . B(S E)$ for all variables in this model were $2.37(1.43), p=.097$ for condition, $-.32(.20), p=.12$ for BMI, and .84 (.50), $p=.09$ for BMI X Condition. To further investigate the $\mathrm{BMI} X$ Condition interaction a spotlight-analysis at one standard deviation above and below mean BMI was conducted. The results of this analysis are depicted in Figure 7.4. At low levels of BMI (-1 SD), participants in the FoodNeu and FoodNeg conditions did not differ in their probability of choosing chocolate (FoodNeu $=.99$, FoodNeg $=.95, B=-1.43$, SE $=1.16, p=$ .37). At high levels of $\mathrm{BMI}$, there was a trend-significant effect indicating a higher probability of choosing chocolate for those in the FoodNeg (1.0) compared to the FoodNeu condition (.82), $B=6.18, S E=3.4, p=.07$. These results suggest a conditioned behavioural effect for participants with higher levels of BMI.

\section{CS Liking Emotional valence}

The Condition XTime interaction was not significant, $F(1,34)=.46, p=.50$, but a significant Condition XTime X EE interaction, $F(1,34)=6.06, p=.019, \eta^{2}=.14$, indicated that EE scores influence the change in emotional valence over time differently for each condition. A subsequent moderated regression and spotlight-analysis with simple slope testing at one standard deviation below or above the mean EE-score was conducted on the emotional valence difference score (baseline - post-conditioning) and is plotted in Figure 7.5. Centred Condition and Emotional Eating scores were entered in Block $1\left(R^{2}=.027, p=.61\right)$, and their interaction was added in Block $2\left(R^{2}=.238, p=.003\right)$. This analysis revealed a significant Con- 


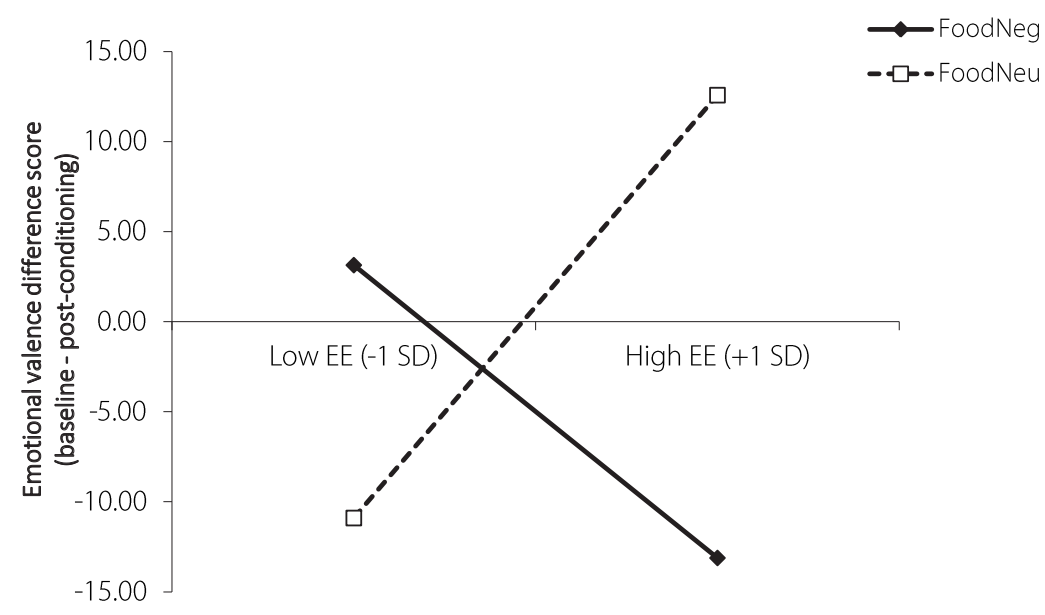

Figure 7.5 Emotional valence difference scores as a function of condition and low and high emotional eating scores (1SD and +1 SD from the mean). Positive scores indicate an increase in unpleasantness of experiencing negative emotions from baseline to post-conditioning, while negative scores reflect a decrease in unpleasantness.

dition X Emotional Eating interaction, $B(S E)=33.52$ (10.60), $\beta=.69, t(39)=3.16, p=.003$. More specifically, low emotional eaters (-1 SD) in both conditions did not differ on the change in unpleasantness ratings after experiencing negative emotions, B (SE) = -14.04 (8.82), $t$ (39) $=-1.59, p=.12$. On the other hand, participants scoring high on emotional eating ( $+1 \mathrm{SD})$ responded differently to the experience of negative emotions, depending on condition, $B$ $(\mathrm{SE})=25.69$ (8.85), $t(39)=2.90, p=.006$ : when negative emotions were not accompanied by eating chocolate, high emotional eaters rated experiencing negative emotions as more unpleasant, and significantly more so than low emotional eaters, B (SE) = 19.80 (7.17), t (39) $=2.76, p=.009$. When negative emotions were paired with eating chocolate, however, the unpleasantness ratings decreased, and were marginally lower than those of low emotional eaters, $B(S E)=-13.71(7.80), t(39)=-1.76, p=.089$.

\section{DISCUSSION}

We investigated whether negative emotional states can act as conditioned appetitive stimuli that elicit cue reactivity, and we expected the appetitive conditioning of emotional mood cues to be stronger than the appetitive conditioning of neutral mood cues. We found 
clear evidence of appetitive conditioning: in both conditions, participants successfully learned to expect and desire foods when confronted with a CS+ while they did not expect and desire foods while confronted with the CS-. This study again shows how relatively easy it is to learn cued desires to eat. However, contrary to expectations, this effect on expectations and desires was not more pronounced in the FoodNeg condition, meaning that the appetitive conditioning using emotional mood cues as CS+ was not stronger, quicker or easier than the conditioning using neutral mood cues as CS+. Though both the negative and the neutral emotional states rather easily elicited cued expectations and desires, such an effect was not found for salivation: significant differences in salivation in response to the CS+ and CS- were not found. The behavioural data, the choice task, did however show the predicted effect, with participants in the FoodNeg condition being more inclined to choose chocolate than participants in the FoodNeu condition. A closer look at these data revealed that while at low levels of BMI the probability of choosing chocolate was equal in both conditions, participants higher in BMI chose the chocolate reward more frequently than the monetary reward compared to participants lower in BMI and to the condition in which neutral emotions were associated with the eating. Of note is that in the current design, participants in both conditions experienced only a negative stimulus before the choice task, limiting interpretation of the choice-effect. Adjusting the design so that half of the participants in both conditions are presented with a negative stimulus and the other half with a neutral stimulus could reveal whether the conditioned effect of choosing chocolate is as strong for the FoodNeu as for the FoodNeg condition. Differences in the emotional valence of emotions in the FoodNeg condition were also predicted: if appetitive conditioning with negative emotional states as the CS+ is successful, and CS+ liking occurs, negative emotions should be evaluated as less negative after conditioning. We found that self-reported emotional eating scores significantly influenced the emotional valence of negative emotions after appetitive conditioning: high emotional eaters reported a decrease in experienced unpleasantness of negative emotions after repeated exposure to negative emotions when these emotions were followed by the eating of chocolate.

When examining the expectancy ratings, it is interesting to note that expectancies to receive food differ at the first trial. This might be due to the awareness of emotional eating in the general public: the coverage of emotional eating in the media means that most people will be familiar with the topic and endorse the idea that an association between negative emotions and eating exists, which translated to specific expectancies during the first conditioning trial. The data on salivation are in line with some other studies from our lab that assessed salivary responses in appetitive conditioning (Bongers et al., 2015; van den 
Akker et al., 2014; Van den Akker et al., 2013). None of these studies found clear evidence for a conditioned salivation response. Possibly salivation responses are vulnerable to crossover effects when measuring responses to CS+ and CS- within subjects and using cotton dental rolls. A recent study by Meyer et al. (2015) measured swallow responses according to the method of Nederkoorn, Smulders, and Jansen (1999) instead of using cotton rolls. They found conditioned responding (increased swallowing in response to the CS+) but only in obese and not healthy-weight participants. Together with our findings of an increased preference of chocolate over money which was present in the sample with higher BMI, these results might suggest that obese individuals are more susceptible to cue-induced responding than their healthy-weight counterparts. This idea fits with numerous previous studies that have demonstrated an association between weight status and food cue reactivity, in which higher BMI is related to increased food cue reactivity like a stronger attention bias for food (Bongers et al., 2014; Castellanos et al., 2009; Nijs, Muris, Euser, \& Franken, 2010; Werthmann et al., 2011), stronger desire to eat after exposure to the sight or smell of food (Ferriday \& Brunstrom, 2011; Tetley, Brunstrom, \& Griffiths, 2009), more salivation (Epstein, Paluch, \& Coleman, 1996), increased brain reward activity (Pursey et al., 2014) and increased cued intake (Jansen et al., 2003; Jansen et al., 2008).

Of interest is our finding that, after being followed by the eating of chocolate, negative emotions were rated as less unpleasant (i.e., 'liked' more) by emotional eaters only. This finding seems to be in line with affect regulation (Ganley, 1989) and escape theories (Heatherton \& Baumeister, 1991), which propose that food intake can serve to improve mood, either by reducing negative feelings (affect regulation) or by creating a distraction from aversive self-awareness (escape theory). However, the current design withholds us from drawing any conclusions regarding eating-induced changes in mood. Inclusion of a third mood assessment after chocolate consumption could provide more insight into the mood-improving effects of food intake. Also, though affect regulation and escape theory do predict a change in mood, they do not necessarily predict changes in the experienced valence of negative emotions. A more parsimonious explanation for the demonstrated change in the valence of negative emotions is the occurrence of evaluative conditioning. Evaluative conditioning refers to a valence change of the conditioned stimulus, i.e., mood in the current study, due to its co-occurrence with the unconditioned stimulus, i.e., the tasting of pleasant foods. The current findings are in line with previous studies demonstrating increased liking of a neutral cue (CS) after it was repeatedly paired with palatable foods (Bongers et al., 2015; Papachristou et al., 2013; van den Akker et al., 2014; van den Akker et al., 2015; Van den Akker et al., 2013; Van Gucht et al., 2010). Similar to the increased liking of vases, trays or jewelry 
boxes in those earlier studies, the increased liking (or lower disliking) of negative emotions (CS) as observed in the current study could be explained in terms of evaluative conditioning. Remarkably, this change in emotional valence of negative emotions was only observed in participants scoring high on self-reported emotional eating. Why this change is specific to this group is a question for future research. Emotional eating questionnaires should be interpreted with some caution. Although the data clearly show an effect of DEBQ-EE scores on change in unpleasantness ratings, high scorers on emotional eating scales do not necessarily represent true emotional eaters. Recent studies have cast doubt on this assumption (Adriaanse, de Ridder, \& Evers, 2011; Bongers et al., 2013a; Bongers et al., 2013b; Bongers, de Graaff, \& Jansen, 2015; Brogan \& Hevey, 2013; Conner, Fitter, \& Fletcher, 1999), and it has been proposed that high-scoring individuals are instead characterized by a more general eating concern or constant worrying about eating (Adriaanse et al., 2011; Jansen et al., 2011b).

One question the current design cannot account for is whether it is truly a feeling that has become a conditioned stimulus or whether it is the knowledge that something sad is about to occur that elicited conditioned responding. In the design of the study, we focused strongly on feelings. Participants were instructed before every stimulus to concentrate on how the stimulus would make them feel, and when rating their expectancy and desire, the question was framed in such a way that participants had to focus on how they felt and answer the questions depending on this feeling. However, for all of the stimuli it was most likely clear from early on whether they were negative or neutral (e.g., the first image of the film clip being of a man dying, or being given the instructions to read the sad memory). Thus, after some pairings, participants might have been able to know a stimulus would be sad at first sight, leading immediately to the knowledge that chocolate would (or would not) follow, without actually having experienced feelings of sadness (which would develop during the 3 minutes of experiencing the stimulus). Although it would be interesting to design a future study in such a way that conditioned responding to knowledge can be separated from responding to feelings, for example by adding expectancy and desire ratings soon after stimulus onset, both types of responding could be involved in emotional eating. In real life, it is likely that emotional eaters initiate food intake when they experience negative feelings, which trigger conditioned responses. On the other hand, it is also conceivable that the knowledge that something aversive is about to occur is enough to elicit these responses. It might be that the knowledge in itself induces apprehension - a negative feeling - and thus leads to craving and food consumption. An alternative is that conditioned responding occurs in such situations to 'soften the blow' of what is coming; eating palatable food could have a protective effect, and could be a learned precautionary measure. 
The current findings have implications for combating emotional eating. Based on the classical conditioning principle of extinction, individuals should form a CS (i.e., negative feeling) - no US (i.e., eating) contingency, in which the CS does no longer predict the US. The new contingency should become stronger and more prominent than the original CS - US relationship. This can be obtained by repeatedly presenting the CS without it being followed by the US. Cue exposure with response prevention therapies (CERP), in which sight and smell of food are CSs, have proven fruitful in reducing CS - US contingencies in patients with bulimia (Jansen, Broekmate, \& Heymans, 1992; Martinez-Mallén et al., 2007; Toro et al., 2003), overweight children (Boutelle et al., 2011), and overweight adults (Schyns, Roefs, Mulkens, \& Jansen, 2015). It would be worthwhile to investigate whether CERP is also effective with negative emotional states as CSs.

Finally, a limitation of the study is that only healthy young women were included in the sample. Although this enabled us to study appetitive conditioning on emotional states in a homogenous group and made it possible to compare our results directly to the results of previous studies, generalization of the findings to other populations is limited.

To conclude, it appears that negative emotional states do not differ from other stimuli in terms of learning potential, and can thus easily become conditioned stimuli for the intake of high-calorie snack foods. Although this has been theorized before, the current study is the first to experimentally show appetitive conditioned responding to negative emotional states and provides exciting evidence for emotional devaluation of the conditioned stimuli, i.e. negative emotions, in emotional eaters.

\section{ACKNOWLEDGEMENTS}

This study is part of an ongoing project that is financed by the Netherlands Organisation for Scientific Research (NWO): Vici Grant 453.10.006, awarded to Anita Jansen. 

8

General Discussion 

The current dissertation addressed emotional eating. The topics covered in this thesis broadly pertain to three categories: (1) cues that elicit overconsumption in emotional eaters, (2) the role of classical conditioning in emotional eating, and (3) the influence of individual factors on cued responding. Chapter $\mathbf{2}$ and Chapter $\mathbf{3}$ described two studies investigating overeating after positive emotions in emotional eaters, whereas Chapter $\mathbf{4}$ focused on overeating in response to both emotional and external cues. In Chapter 5, cue reactivity in the form of attention bias was studied in relation to impulsivity and weight status. Chapter 6 and Chapter $\mathbf{7}$ focused on the role of classical conditioning in emotional eating. In the current chapter, a summary of the research findings is presented, followed by a discussion of these findings. Attention is then given to implications for clinical practice as well as methodological consideration of the studies included in this dissertation. Recommendations for future research will be made throughout the chapter.

\section{SUMMARY OF THE FINDINGS}

\section{Cue-elicited overeating in emotional eaters}

The studies described in Chapters $\mathbf{2}$ and $\mathbf{3}$ both aimed to investigate the role of positive emotions in emotional eating. Participants underwent a negative, positive, or neutral mood induction and took part in a bogus taste test. In Chapter 2, emotional eaters were identified through a self-report questionnaire. The results showed that individuals scoring high on this questionnaire (i.e., emotional eaters) consumed more food in the positive compared to the neutral condition, and showed higher intake after positive emotions compared to non-emotional eaters. There was no increase of food intake after negative emotions. A 
second aim of the study was to assess changes in mood as a result of eating palatable food. Emotional and non-emotional eaters did not differ in this respect. Both groups reported an increase in mood in the first five minutes of eating, and remained at this mood level during the remaining ten minutes. In Chapter 3, an implicit measure (i.e., single-target IAT) was added to the self-report questionnaire. In this particular IAT, stronger implicit associations between emotions and food are thought to reflect more emotional eating. In this study, emotional eaters defined on basis of self-report did not differ from non-emotional eaters in terms of food intake. Both groups consumed the same amount of food in all conditions. Participants who showed strong associations between positive emotions and food consumed most food after the positive mood induction. Interestingly, the positive mood induction also led to highest food intake in individuals with strong associations between negative emotions and food. Taken together, these studies show that positive moods can also elicit overeating in emotional eaters, perhaps even more so than negative moods.

In Chapter $\mathbf{4}$ emotional eaters were identified based on self-report and on actual eating behaviour in response to negative emotions. A food cue exposure condition was added to the two emotional conditions and the control condition. Emotional eaters were found to consume more food in all conditions, even the control condition, than non-emotional eaters. Furthermore, food intake in emotional eaters did not differ between the experimental and control conditions. Based on these data it is proposed that emotional eaters might be more appropriately termed 'cue-reactive eaters', to reflect their tendency to overeat in response to a variety of cues, not restricted to emotions.

\section{Individual differences: trait food craving, impulsivity and weight status}

Chapter $\mathbf{5}$ focused on a study designed to elucidate the role of trait craving, impulsivity and weight status in cue reactivity, more specifically attention bias. Participants completed a selfreported trait craving questionnaire, self-reported and behavioural impulsivity measures, and a visual search task. Evidence for an attention bias was found in individuals who were obese and displayed high self-reported impulsivity. These participants showed a speeded detection of high-caloric foods compared to healthy-weight impulsive participants.

\section{The role of classical conditioning in emotional eating}

Chapters 6 and 7 focused on two conditioning pathways proposed to be potentially involved in emotional eating. These are 1) facilitation of appetitive conditioning in a negative mood and 2) the development of negative emotional states into conditioned stimuli. In Chapter 6 it was investigated whether a negative mood facilitates appetitive condition- 
ing to neutral stimuli, and whether this is influenced by trait impulsivity. Participants who were brought into a negative or a neutral mood underwent a conditioning procedure, and expectancy and desire to eat, salivation and food intake were measured. Results showed successful appetitive conditioning in terms of expectancy and desire to eat, but a negative mood did not facilitate conditioning. An effect of impulsivity was found with regard to food intake. High impulsive participants consumed less food when presented with the CS+ (i.e., neutral cue paired previously paired with food) compared to the CS- (i.e., neutral cue previously not paired with food).

Chapter 7 reported on the second pathway, in which negative emotions were hypothesized to develop into conditioned stimuli and predictors of food intake. Participants were randomly divided over two conditions, one in which negative feelings were followed by eating chocolate but neutral feelings were not (FoodNeg condition), and one in which this was the other way around (FoodNeu condition). Expectancy and desire to eat were measured, as well as salivation, unpleasantness of experiencing negative emotions, and a chocolate vs. money choice task. The expectancy and desire data showed that negative emotions can indeed develop into conditioned stimuli. Participants who received chocolate when in a negative emotional state reported increased expectancy and desire to eat when feeling negative compared to feeling neutral. A stronger preference for chocolate over food in a negative mood was observed for participants in the FoodNeg condition, but only at high levels of BMI. Furthermore, in emotional eaters, consumption of palatable food during a negative emotional experience seemed to attenuate the effects of that experience. Based on the results of these studies, it seems likely that classical conditioning processes can lead to emotional eating.

\section{GENERAL DISCUSSION}

\section{Cue-elicited overeating in emotional eaters}

The first three studies described in this dissertation (Chapters 2-4) cast serious doubt on the assumption that emotional eaters overeat specifically and exclusively in response to negative emotions. They also (and perhaps even more so) overeat when happy. In fact, emotional eaters could be more accurately described as cue-reactive eaters who overeat upon encountering a variety of cues (see Chapter 4). Interestingly, high scores on selfreported emotional eating were related to different behaviour in all three studies: In the first study, self-reported emotional eaters increased food intake in a positive mood, but not in a 
negative mood; In the second study, emotional eaters did not differ in intake when feeling negative compared to positive, nor did they differ from non-emotional eaters; In the third study, there was evidence for increased intake in emotional eaters after experiencing both negative and positive emotions, as well as after food cue exposure and upon mere presentation of food. The divergent nature of the findings of these three studies is characteristic of all emotional eating research (see Table 1.1 in Chapter 1 for an overview).

\section{Methodological differences}

One explanation for the variety in findings in the field of emotional eating research could be the methodological differences across the studies. A number of different emotional states have been induced in a number of different ways, and also participant samples vary greatly. In fact, in the 38 experimental studies described in Table 1.1, 14 different mood inductions have been used to evoke seven different kinds of negative moods and four kinds of positive moods, in samples with male and female students, male and female adults, individuals with obesity, binge eating disorder or depression, restrained eaters and disinhibited eaters. It could be argued that there may not be a universal mood induction or mood state with which to obtain the strongest effects. Indeed, it seems more likely that there are individual differences in this respect: whereas one individual might experience a desire to eat when watching a sad film, another might feel this way when recalling a sad memory, while a third person's eating behaviour is not affected by sadness but by stress. And while for some individuals the situation that evokes the emotions needs to be personally relevant, for another this may not matter. The role of the arousal level of the induced emotions has also been stressed (Evers, Adriaanse, de Ridder, and De Witt Huberts, 2013). From this perspective, although more complicated to implement, tailored mood manipulations could be a worthwhile addition to future research. Another consideration is that food intake is generally measured after the mood induction, and it can be questioned how long the negative effects of a short lab-induced mood will last, and whether this differs between manipulations.

A second methodological difference lies in the way analyses are carried out. A number of studies that did find evidence for emotional eating restricted their sample to extreme scores; only individuals with extremely high (i.e., $80^{\text {th }}$ percentile) and extremely low (i.e., $20^{\text {th }}$ percentile) emotional eating scores were included in the study (Van Strien, Cebolla, et al., 2013; Van Strien, Herman, Anschutz, Engels, \& de Weerth, 2012; Van Strien, Ouwens, Engel, \& de Weerth, 2014; Van Strien, Roelofs, \& de Weerth, 2013). The lack of consistent findings and the need for extreme scores implies that emotional eating might not be a robust phenomenon. If emotional is eating is (nearly) only found in extreme scorers on self-report 
questionnaire or when using very specific paradigms in very specific samples, it follows that it might not be such a common occurrence as some scientists, the media and general opinion often lead us to believe.

\section{Interpret with caution}

From the conflicting results of earlier studies as well as the studies in this dissertation it becomes clear that self-reported emotional eating questionnaires should be interpreted with caution. Data from studies incorporating such questionnaires varies greatly and evokes the question what it is exactly that is measured by such questionnaires. Apparently, they do not reliably measure actual food intake in response to negative emotions, that is, emotional eating in the original sense of the word. However, understanding the true meaning of a high self-reported emotional eating score is crucial for the interpretation of all studies on this topic. For example, studies that have related emotional eating to depression (Konttinen, Silventoinen, Sarlio-Lähteenkorva, Männistö, \& Haukkala, 2010; Ouwens, Van Strien, \& Van Leeuwe, 2009), BMI (Konttinen, Silventoinen, et al., 2010; Laitinen, Ek, \& Sovio, 2002; Péneau, Ménard, Méjean, Bellisle, \& Hercberg, 2013) or weight gain (Hays \& Roberts, 2008; Koenders \& Van Strien, 2011) should no longer be assumed to have found an association between 'increased food intake in a negative mood' on the one hand and depression, BMI, or weight gain on the other hand. Likewise, experimental studies may not have compared 'individuals who increase food intake in a negative mood' to individuals who do not. However, how these data should be interpreted remains unclear. Suggestions have been made for concerned eaters (Adriaanse, de Ridder, \& Evers, 2011; Jansen, Nederkoorn, et al., 2011), uncontrolled eaters (Vainik, Neseliler, Konstabel, Fellows, \& Dagher, 2015), and cue-reactive eaters (this dissertation). All three interpretations are similar in the sense that they propose there is more to emotional eating than eating in response to negative emotions, and the interpretations are not mutually exclusive. However, they are not in full accordance either: whereas concerned eaters are characterized by a continuous concern about and preoccupation with eating behaviour and is cognitive in nature, uncontrolled and cue-reactive eating are descriptive of actual eating behaviour. How concerned eaters and uncontrolled and cue-reactive eating are exactly related is a topic for future research.

Because previous studies have resulted in different outcomes with regard to self-report questionnaires (i.e., high scores are predictive of increased food intake after negative emotions, increased intake after positive emotions, or no increase at all), it remains to be seen whether the self-report results from Chapter 4 (i.e., increased food intake in a negative mood, positive mood, after food cue exposure and after a control condition in self-reported 
emotional eaters) can be replicated. In support though, there are indications that emotional eating is not a clearly demarcated phenomenon. Studies have found correlations between emotional and external eating (Jansen, Nederkoorn, et al., 2011; Turner, Luszczynska, Warner, \& Schwarzer, 2010; Van Strien, Frijters, Bergers, \& Defares, 1986) and eating patterns of emotional eaters cannot always be discriminated from those of other types of eaters (Jansen, Nederkoorn, et al., 2011). Furthermore, the results from the behavioural measure speak in favour of such labelling. Participants who display increased food intake after negative emotions do so after other cues as well. It could be argued that the behavioural measure is a more reliable measure of emotional or cue-reactive eating than scores on questionnaires, whose validity has been called into question repeatedly. Thus, these data provide compelling evidence for the idea that 'emotional eating' does not cover the extent of overeating in what are currently called 'emotional eaters'. However, because the control condition in our study elicited the same eating behaviour as the experimental conditions, it is unclear whether these cue-reactive eaters always consume more food than non-cue-reactive eaters, or whether the control condition unintentionally served as a fourth experimental condition (i.e., cue). In case of the former, it would mean that some individuals will (nearly) always consume food when there is the chance while others do not, and 'eaters' vs. 'noneaters' might be a suitable distinction as well.

\section{Where does dietary restraint fit in?}

A final question to address is how dietary restraint fits in the suggested distinction between cue-reactive and cue-non-reactive eaters. The discrimination between emotional, external and restrained eaters was introduced three decennia ago, based on suggestions that restrained eaters who are successful at restricting food intake should be distinguished from restrained eaters who tend to break their diet and overeat. Following this reasoning, emotional and external eating are forms of disinhibition, and are considered to be behaviours that can only occur as a reaction to inhibition, or restraint. However, equal to letting go of the idea that emotional and external eaters are fundamentally different, perhaps a similar change in perspective on dietary restraint is called for. It does not seem all that unfeasible that in our current obesogenic society with its abundance of palatable food and food cues, dietary inhibition is not a prerequisite for overeating (Westenhoefer, 1991; Yeomans \& Coughlan, 2009). Even an individual who does not restrict food intake can be enticed to overeat if the right cues are present. In addition, taking a conditioning perspective on how cues elicit eating desires, conditioned responding to food cues would not require dietary restraint to be present. Thus, in terms of cue-reactive and non-cue-reactive types of eaters, eating 
restraint could be present (or not) in both types: a restrained eater who is non-cue-reactive would encounter only few challenges, while a non-cue-reactive and non-restrained eater would likely not be concerned about food at all. An individual who is highly responsive to food cues might not be bothered by this if there are no self-imposed eating restrictions. On the other hand, someone who does adhere to such restrictions and responds easily to cues would have to put more effort in staying in control. Finally, whereas the original distinction between restrained, emotional and external eating presumed emotional or external eating to be a consequence of restraint, it can also be argued that unwanted cue-reactive overeating is a precursor to restriction of intake (Johnson, Pratt, \& Wardle, 2012).

\section{Conclusions and future directions}

From the work presented in the first three chapters of this dissertation, it becomes apparent that although it may not be clear what exactly emotional eating is, it is clear what it is not: emotional eating is not merely overeating in a negative mood. Instead, emotional eaters seem more likely to overeat in response to a variety of cues, including but not limited to negative emotions. These findings should be taken into account in the interpretation of findings based on self-reported emotional eating scores. An interesting next step would be to pursue the idea that 'emotional eating' reflects only a small part of a larger issue regarding overeating. It seems highly unlikely that there are individuals who overeat only in response to negative emotions and are perfectly able to control their food intake under other challenging circumstances. Instead, emotional eaters are better described as cue-reactive eaters. Future studies could assess whether the proposed distinction of cue-reactive vs. non-cue-reactive eaters is valid, and if so, how restrained eating is related to these two eater types. Do cue-reactive eaters increase food intake whenever the opportunity for eating arises (i.e., are there simply eaters and non-eaters?), or is it conditional on specific cues? Are these cues the same for every individual? How does cue-reactive eating develop; have the cues been conditioned to food intake? Are some individuals easier to condition to appetitive cues than others, and are there specific traits that influence this? How can we reliably assess cue-reactive eating? Should restrained eating be considered as a type of eater separate from cue-reactive eaters, or is it a trait that can be present in both cue-reactive and non-cue-reactive eaters?

\section{The role of classical conditioning in emotional eating}

Classical conditioning processes have long been suggested to underlie overeating (Jansen, 1990, 1998; Jansen, Havermans, \& Nederkoorn, 2011; Jansen, Van den Hout, De Loof, 
Zandbergen, \& Griez, 1989; Wardle, 1990), and all kinds of cues have the ability to become predictive of food intake. As such, chronic overeaters (e.g., cue-reactive eaters) may have developed conditioned associations between eating and a multitude of cues, both internal and external, leading them to overeat in a variety of situations. The following paragraphs focus on the role of emotional states in classical conditioning.

\section{Conditioning and mood states}

Based on findings that high-calorie foods have strong rewarding properties (Coletta et al., 2009; Macht \& Dettmer, 2006; Macht \& Mueller, 2007; Small, Zatorre, Dagher, Evans, \& JonesGotman, 2001) which might be extra rewarding and thus relevant for people who find themselves in a negative emotional state (Baker, Piper, McCarthy, Majeskie, \& Fiore, 2004; Hepworth, Mogg, Brignell, \& Bradley, 2010), it was hypothesized that a negative mood would lead to facilitated conditioning compared to a neutral mood. Both studies (Chapters 6 and 7) provided convincing evidence for appetitive conditioning. Participants learned to expect food and their desire to eat the food increased when presented with the CS+ stimulus (i.e., one particular vase or emotional state), but not when presented with the CS- stimulus (i.e., another vase or emotional state). However, the hypothesis regarding mood states was not confirmed. Negative mood neither led to faster, easier, or stronger appetitive conditioning of neutral cues (Chapter 6), although participants in a negative mood did report a higher desire to eat when facing both the conditioned cue (CS+) and a large quantity of palatable food. In addition, negative mood states did not develop more quickly into conditioned stimuli than neutral mood states (Chapter 7). With little apparent effect of a negative mood states, it is interesting to consider if positive mood states would differently affect conditioning. Given the evidence for the ease with which external and negative internal cues can develop into conditioned stimuli, it seems very likely that positive emotional states will not differ in this respect. Of interest is that findings from studies on fear conditioning (Stegen, De Bruyne, Rasschaert, Van de Woestijne, \& Van den Bergh, 1999; Van Den Bergh, Kempynck, Van De Woestijne, Baeyens, \& Eelen, 1995; Van den Bergh, Stegen, \& Van de Woestijne, 1997, 1998) suggest that similarities in valence (e.g., the CS and US being both positive in valence) and confirmed expectancies (e.g., higher inclination to expect a positive US after a positive CS) are important factors that can promote CS-US learning. Because positive mood or positive stimuli are congruent with a palatable food US in terms of valence and positive CSs are likely to raise positive expectations, positive moods may have the ability to expedite the conditioning process. 


\section{Aetiology or maintenance?}

The results from Chapter 7 provide evidence for the idea that emotional states can act as conditioned stimuli, and thus point to a role for classical conditioning in emotional eating. It is interesting to consider whether classical conditioning is involved in the aetiology of emotional eating, the maintenance of emotional eating, or both. Imagine that a child is repeatedly pacified with food when in distress (e.g., feeling anxious, sad, or angry). Classical conditioning can take place and after some time the child will automatically and immediately desire food when experiencing such distress: emotional eating has developed. Indeed, a number of studies (Blissett, Haycraft, \& Farrow, 2010; Braden et al., 2014; Rodgers et al., 2013) and theories (Bruch, 1964) consider emotional eating as something that can develop during childhood, though the precise processes are not specified by these authors. It is also conceivable that emotional eating develops later in life. Imagine an adult woman who hears a friend say that she always feels better after eating sugar candy. Next time this woman feels bad, she takes some sugar candy, and notices that it improves her mood. If she engages in this behaviour repeatedly, a negative feeling will automatically lead to eating desires and food intake. In both examples, classical conditioning is a mechanism involved in the development of the emotional eating.

Although eating may give a short respite from negative feelings (Kenardy, Butler, Carter, \& Moor, 2003; Macht \& Mueller, 2007; Tuomisto, Tuomisto, Hetherington, \& Lappalainen, 1998), it has also been argued to have particularly negative consequences on the longer term. The relief from negative emotions is often followed by even stronger negative feelings, such as guilt, shame or distress (Haedt-Matt \& Keel, 2011; Hendy, 2012; Macht, Gerer, \& Ellgring, 2003; Solomon, 2001; Wansink, Cheney, \& Chan, 2003), and emotional eating has been related to aversive outcomes such as weight gain (Hays \& Roberts, 2008; Koenders \& Van Strien, 2011). Even when someone acknowledges that the costs of emotional eating outweigh the benefits, attempts to stop the behaviour are not always successful. This illustrates the possible maintaining effect of classical conditioning on emotional eating. Conditioned responses are hard to overcome, and increase in strength with every CS - US pairing.

\section{Food and feelings}

The notion that eating diminishes negative feelings is the central tenet of affect reduction theories (Ganley, 1989), but this supposition has received mixed support (Haedt-Matt \& Keel, 2011). An alternative explanation has been provided by escape theory (Heatherton \& Baumeister, 1991), which states that individuals engage in eating to escape from aversive self awareness. Instead of focusing attention on the self, distraction takes place by means 
of focus on something in the environment, such as food. Whereas affect reduction theories assume that a negative mood is permanently alleviated through food intake, escape theory proposes that this decrease in negative effect lasts for as long as the individual engages in eating (i.e., distraction). When food intake is terminated and focus returns to the self, negative mood will increase again. The emotional valence data from Chapter 7 suggests a third mechanism through which eating can affect mood. These data show that in selfreported emotional eaters, consumption of chocolate during a negative emotional state decreases the unpleasantness of such states. Thus, the consumption of highly rewarding foods (US) might reduce the negative valence of the food-predicting emotions (CS) and therefore eating possibly has a protective effect when experiencing negative emotions. As such, instead of reaching a maximum level of negativity that is subsequently reduced by eating, as proposed by affect reduction and escape theories, food intake previous to or concurrent with a negative emotional experience might prevent negative mood from reaching its highest level.

\section{Conclusion and future directions}

Two main conclusions can be drawn from the two studies on emotional eating and classical conditioning presented in this dissertation: (1) Negative emotional states can act as conditioned stimuli and elicit cue- reactive behaviour, as shown by increased eating expectancies and desires and choice behaviour, and (2) negative mood does not facilitate (or impair) appetitive conditioning to neutral cues. A tentative third conclusion is that appetitive conditioning of negative emotions predicting food rewards might lead to changes in the emotional valence of negative emotions. It would be highly interesting to investigate whether inducing positive mood states differs in any way from inducing negative or neutral mood states. It is clear that positive moods can induce overeating. If these kinds of moods can also lead to faster or stronger conditioned responding, regardless of whether this would be as an occasion setter or CS, these specific moods could be given extra attention in treatment to prevent or reduce positive-mood-induced overeating. Furthermore, knowledge about conditioned responding in positive moods could provide additional insight into the mechanisms behind conditioned overeating.

Likewise, the studies could be repeated using other participant samples, such as obese individuals, to investigate whether they show different conditioned responding than healthy students. It seems likely that differences would indeed emerge between the two groups. Previous studies have demonstrated heightened food cue reactivity in obese individuals (Ferriday \& Brunstrom, 2011; Jansen et al., 2003; Sobik, Hutchison, \& Craighead, 2005; 
Tetley, Brunstrom, \& Griffiths, 2009), which could be reflected in faster food-cue related conditioned responding. The only study on human appetitive conditioning that has taken weight into account found evidence for stronger conditioned swallowing responses in obese compared to lean participants (Meyer, Risbrough, Liang, \& Boutelle, 2015). The effect of weight on other conditioning outcomes (e.g., expectancies, desires, and food intake) has not yet been investigated. Whether heightened cue reactivity, and possibly facilitated conditioning, is a precursor or a consequence of obesity is a question that remains unanswered. Nevertheless, if obese individuals indeed show stronger conditioning than healthy-weight individuals, this might provide insight into the mechanisms that lead to the development or maintenance of obesity.

With regard to Chapter 6, future studies could investigate negative emotions as occasion setters for food intake. An occasion setter is a stimulus that modulates an association between a CS and a US, and could be involved in emotional eating. If a neutral stimulus becomes associated with food intake while the individual is in a negative mood, this stimulus will only elicit conditioned responding to the cue when the negative mood is present. The negative mood sets the occasion for the conditioned stimulus to be reinforced. This would also imply that emotional eating only takes place when the mood is negative and the conditioned cue is present; the presence of a negative mood is necessary but not sufficient. Whether this reflects emotional eating in real life is an empirical question. Not much is known about the places where individuals engage in emotional eating. If this is for example mostly at home, it could be that that particular context (i.e., the home environment) has been conditioned to food intake when in a negative mood. This could also contribute to an explanation for the absence of emotional eating in many studies. The occasion setting mood is present, but the conditioned cue is not. Conversely, the right cue could be present whereas the required mood is not. In studies that do find evidence for emotional eating, there might have been the right combination of mood and cues. To test negative mood states as occasion setters for conditioned responding, all participants would have to undergo conditioning trials in randomly alternating negative and neutral mood states. One CS (e.g., the vase with flowers as used in the original study) is then paired with food intake in a negative mood, but not in a neutral mood. During and after the procedure, conditioned responding should only occur when the participant is in a negative mood and in the presence of the CS. Such a design would go beyond the study set-up in Chapter $\mathbf{6}$, which focused on the facilitating effect of negative mood on learning, but did not include mood as a CS. 
Chapter 7, which reports on negative emotional states as conditioned stimuli, paves the road for follow-up studies on extinction of the CS - US (i.e., negative emotional state - food intake) association. In terms of extinction, inhibitory learning of the original association is the central mechanism (Bouton, 1993). Individuals should form a CS (e.g., negative mood or smell of tasty food) - no US (i.e., no eating) contingency, in which the CS does no longer predict the US. The new contingency should become stronger and more prominent than the original CS - US relationship, in which a cue like mood state or food smell signalled eating. This can be obtained by repeatedly presenting the CS without it being followed by the US. Promising results in this respect have been obtained with various external cues in experimental lab studies (Papachristou, Nederkoorn, Beunen, \& Jansen, 2013; Van den Akker, Havermans, Bouton, \& Jansen, 2014; Van den Akker, Havermans, \& Jansen, 2015; Van Gucht, Baeyens, Vansteenwegen, Hermans, \& Beckers, 2010; Van Gucht, Vansteenwegen, Beckers, \& Van Den Bergh, 2008; Van Gucht, Vansteenwegen, Van den Bergh, \& Beckers, 2008) and the sight and smell of food in clinical studies (Boutelle et al., 2011; Jansen, Broekmate, \& Heymans, 1992; Martinez-Mallén et al., 2007; Schyns, Roefs, Mulkens, \& Jansen, submitted for publication; Toro et al., 2003). It should be noted that whereas clinical studies have successfully decreased eating binges and eating desires, eating desires are more resistant to extinction in lab studies. These differences could be due to the setting (lab vs. clinical), duration (8 trials vs. intensive multiple-session exposure) and participant sample (student vs. overweight or eating-disordered patients). Thus, future studies could assess the effectiveness of extinction in reducing conditioned appetitive responding to emotional states. If this procedure is identified as effective in scientific studies, a translation to clinical practice can follow.

\section{Individual differences}

One topic that received limited attention in this dissertation is individual differences. Are there any personality traits or other individual characteristics that can influence responsiveness to cues in the domain of food and appetite? The results from Chapter $\mathbf{5}$ suggest that there are. A combination of being obese and impulsive was found to be related to speeded detection of high-caloric food. The speeded detection in this study reflects attention bias, a form of cue reactivity. The results are somewhat in line with several earlier studies that have related both impulsivity (Hou et al., 2011; Lattimore \& Mead, 2015) and overweight or obesity (Castellanos et al., 2009; Nijs, Muris, Euser, \& Franken, 2010; Werthmann et al., 2011) to stronger attention bias, although the specifics of this association remain open to debate. For example, whereas previous studies have highlighted influences of impulsivity and weight separately, this dissertation revealed the importance of a combination of these 
factors. Likewise, in contrast to Chapter 5, Lattimore and Mead (2015) reported slowed disengagement from food cues in high impulsive participants, but speeded detection in low impulsive participants. Finally, the nature of attention bias in obese participants differs over studies (Castellanos et al., 2009; Nijs et al., 2010; Werthmann et al., 2011).

Chapter 6 revealed that participants scoring high on self-reported impulsivity consumed less food during a taste test when a cue that was paired with eating during a prior conditioning procedure was present. However, in an earlier appetitive conditioning study, impulsivity was found to be related to increased intake in presence of the food-paired cue (Van den Akker, Jansen, Frentz, \& Havermans, 2013). Although most evidence points in the direction of a strong positive association between impulsivity, cue reactivity and increased food intake (for an overview see Guerrieri, Nederkoorn, \& Jansen, 2008; Van den Akker, Stewart, Antoniou, Palmberg, \& Jansen, 2014) and is in line with the latter findings, not all studies reach the same conclusion (Larsen, Hermans, \& Engels, 2012; Lattimore \& Mead, 2015).

With respect to BMI, Chapter 7 showed a conditioned preference of chocolate over money in a choice task for higher levels of BMI. These data indicate that whereas participants with lower BMI's were not influenced by the conditioning procedure in terms of actual subsequent food-related behaviour, participants higher in BMI were. Taken together with recent findings that showed a conditioned swallowing response in obese but not healthyweight participants (Meyer et al., 2015), this might suggest that a higher BMl is related to stronger cue-induced responding after conditioning.

It would be a logical next step to extend the findings on impulsivity and weight status to the other studies in this dissertation. Considering the ample evidence for a positive association between impulsivity, cue reactivity and overweight/obesity (Guerrieri et al., 2008; Van den Akker, Stewart, et al., 2014), it would be of interest to investigate whether the general cue-reactive responding to food cues as reported in Chapter $\mathbf{4}$ is perhaps more pronounced in impulsive compared to non-impulsive individuals or obese compared to healthy-weight individuals. With regard to appetitive conditioning, as discussed in the previous section, future studies could focus on whether obese individuals respond differently to an appetitive conditioning procedure with external or internal cues than their healthy-weight counterparts, in terms of self-reported expectancy and desire ratings as well as physiological and behavioural outcomes. 


\section{CLINICAL IMPLICATIONS}

Emotional eating is not an eating disorder in and of itself, but rather a specific eating style that can be present in eating disorders such as anorexia nervosa (Ricca et al., 2012), bulimia nervosa (Ricca et al., 2012), binge eating disorder (Eldredge \& Agras, 1996; Pinaquy, Chabrol, Simon, Louvet, \& Barbe, 2003), and obesity (Geliebter \& Aversa, 2003; Hays \& Roberts, 2008), as well as in the general non-eating disordered population (Laitinen et al., 2002; Van Strien, Herman, \& Verheijden, 2009). The golden standard for treatment of eating disorders and eating-related pathology is cognitive behavioural therapy (CBT) (Hay, 2013; Wilson, Grilo, \& Vitousek, 2007). CBT makes use of cognitive and behavioural strategies to change undesired eating-, weight-, and shape-related behaviours and thoughts. One of the strategies that could be - but rarely is (Fairburn, 2008) - incorporated in CBT to reduce overeating is cue exposure with response prevention (CERP). Based on the principles of extinction as explained earlier (i.e., inhibition of the CS-US association, and creation of a stronger CS - no US association), during CERP, individuals are exposed to exactly those cues that elicit a desire to eat and which are usually followed by eating. However, CERP does not allow for food intake to occur. Repeatedly presenting food cues without actual food intake will ultimately extinguish the desire to eat elicited by that cue. CERP has been proven fruitful in reducing CS - US contingencies in patients with bulimia (Jansen, 1990; Jansen et al., 1992; Jansen et al., 1989; Martinez-Mallén et al., 2007; Toro et al., 2003), overweight children (Boutelle et al., 2011), and overweight adults (Schyns et al., submitted for publication). So far, studies on CERP have focused on the sight, smell, and taste of food as CSs, and have been successful in reducing for example eating expectancies, eating binges and food intake.

Based on the findings from Chapter 7, which suggest that negative emotional states can also develop into CSs and elicit cue reactivity, CERP could be applied to emotional states as well. As with exposure to food smells and tastes, clients could be repeatedly exposed to the emotional states that trigger overeating, without receiving the opportunity to eat. Over time, the contingency between emotions and eating should become inhibited, and overruled by a stronger emotion - no eating contingency. Furthermore, given the results from Chapters 2-4 that emotional eaters do not overeat merely in response to negative emotions, it could be useful for therapists to conduct CERP on all salient cues that elicit overeating in a certain individual. Addressing only one of the conditioned cues would mean addressing only a small part of a much larger problem. Thus, for the treatment of emotional or cue-reactive eating, all cues identified by the patient and the therapist as triggers for the 
individual's overeating could be subjected to exposure with response prevention (see for example Jansen, 1998).

\section{METHODOLOGICAL CONSIDERATIONS}

Some methodological issues should be taken into account when interpreting the present results and its implications.

First, there are some considerations that are applicable to most of the presented studies. All studies with the exception of Chapter $\mathbf{5}$ included only healthy (female) students as participants. As a consequence, results cannot be generalized to other populations of interest, such as individuals with overweight or obesity, individuals with clinically relevant disordered eating symptoms, or males. It would be of interest to investigate for example whether obese individuals differ from healthy-weight individuals in terms of appetitive conditioning to emotional cues, or whether males respond similarly to food cues as females. Another issue regarding more than one study (Chapters $\mathbf{2}, \mathbf{3}, \mathbf{4}$ and $\mathbf{6}$ ) is the assessment of food intake in a lab setting. Although the lab provides a controlled environment for experimental research, the circumstances under which food consumption takes place are not natural and therefore might have affected intake - though this is the same in all conditions. Finally, the experimental manipulation and an emotional eating questionnaire were administered within one test session in the studies described in Chapters $\mathbf{2 , 3} \mathbf{3} \mathbf{6}$ and 7. The order in which these components are presented should be carefully considered because of mutual influences. In the studies described here, one cannot rule out the possibility that the experimental manipulation influenced responses on the questionnaire.

The second issue pertains to mood inductions, and is relevant to Chapters $\mathbf{2 , 3 , 4 , 6}$ and 7. It is difficult, if not (ethically) impossible, to elicit immediate and unique emotions. Emotions grow stronger over time, and reach their peak after seconds or even minutes. They can for example not be induced by a single stimulus that lasts only milliseconds and has the ability to reach peak emotional levels within that time. It would be ideal, especially for a study as described in Chapter $\mathbf{7}$ in which emotional states acted as conditioned stimuli, if emotions could be evoked in such way. In addition, induced emotions likely never consist of just that one unique intended emotion. When participants are instructed to think of a sad memory, this memory could evoke a number of other feelings associated with that particular memory, creating a mood induction that is (slightly) different for each participant. 
Even watching a sad film clip that is similar for everyone could elicit different additional emotions in every participant, dependent on personal experiences.

Third, to bypass issues with self-reported emotional eating, two studies incorporated additional measures to assess emotional eating. An IAT was used to identify emotional eaters in Chapter 3, and actual emotional eating behaviour was taken into account in Chapter 4. It should be noted that although both measures have high face validity, they have not been otherwise validated.

The fourth point concerns the effect of impulsivity on cue reactivity (Chapter 5) and learning (Chapter 6). While validated measures of impulsivity were used in both studies, it has repeatedly been noted that impulsivity is a multidimensional construct that can be conceptualized in different ways (Dawe \& Loxton, 2004; De Wit, 2009; Whiteside \& Lynam, 2001). In addition, self-report and behavioural measures of impulsivity have been reported to correlate only weakly or not at all (Enticott, Ogloff, \& Bradshaw, 2006; Marsh, Dougherty, Mathias, Moeller, \& Hicks, 2002; Reynolds, Ortengren, Richards, \& De Wit, 2006). Thus, it remains uncertain whether similar results would be obtained if these studies were repeated with a different conceptualization of impulsivity or by implementing different impulsivity measures.

\section{CONCLUSION}

The main focus of this dissertation was on the type of cues that elicit overeating in emotional eaters, and how classical conditioning processes are involved in emotional eating. In addition, attention was given to individual factors influencing cue reactivity. The results from the presently conducted studies suggest that emotional eating is not the clearly demarcated issue of overeating in response to negative emotions that it is often thought to be. Instead, it appears that emotional eaters are inclined to overeat in response to a variety of other cues as well, including positive emotions and food cue exposure. Furthermore, classical conditioning is likely to play a role in emotional eating, as shown by the finding that negative emotional states can act as conditioned stimuli and easily elicit cue reactivity. Finally, individual differences such as weight status and impulsivity could influence cued responding and classical conditioning. Future studies could elucidate the role of individual characteristics in more detail, further investigate the proposed distinction of cue-reactive vs. non-cue-reactive eaters, and look into extinction of conditioned responding to emotional states. 


References 

Abramson, E. E., \&Wunderlich, R. A. (1972). Anxiety, fear and eating: A test of the psychosomatic concept of obesity. Journal of Abnormal Psychology, 79(3), 317-321.

Adriaanse, M. A., de Ridder, D. T. D., \& Evers, C. (2011). Emotional eating: Eating when emotional or emotional about eating? Psychology \& Health, 26(1), 23-39.

Agras, W. S., \& Telch, C. F. (1998). The effects of caloric deprivation and negative affect on binge eating in obese binge-eating disordered women. Behavior Therapy, 29(3), 491-503.

Arnow, B., Kenardy, J., \& Agras, W. S. (1995). The Emotional Eating Scale: The development of a measure to assess coping with negative affect by eating. International Journal of Eating Disorders, 18(1), 79-90.

Attwood, A. S., O'Sullivan, H., Leonards, U., Mackintosh, B., \& Munafò, M. R. (2008). Attentional bias training and cue reactivity in cigarette smokers. Addiction, 103(11), 1875-1882.

Baker, T. B., Piper, M. E., McCarthy, D. E., Majeskie, M. R., \& Fiore, M. C. (2004). Addiction motivation reformulated: an affective processing model of negative reinforcement. Psychological Review, 111(1), 33.

Bates, J., \& Adams, D. (1968). The influence of mental stress on the flow of saliva in man. Archives of oral biology, 13(5), 593-596.

Beck, A., Steer, R., \& Brown, G. (1996). Manual for the beck depression inventory-II. Psychological Corporation. San Antonia, TX.

Ben-Aryeh, H., Roll, R., Kahana, L., Malberger, E., Szargel, R., \& Gutman, D. (1985). Saliva as an indicator of stress. International Journal of Psychosomatics, 32(3), 3-8.

Bennett, J., Greene, G., \& Schwartz-Barcott, D. (2013). Perceptions of emotional eating behaviour. A qualitative study of college students. Appetite, 60, 187-192.

Birch, L., McPhee, L., Sullivan, S., \& Johnson, S. (1989). Conditioned meal initiation in young children. Appetite, 13(2), 105-113.

Blissett, J., Haycraft, E., \& Farrow, C. (2010). Inducing preschool children's emotional eating: relations with parental feeding practices. The American Journal of Clinical Nutrition, 92(2), 359-365.

Boddez, Y., Baeyens, F., Luyten, L., Vansteenwegen, D., Hermans, D., \& Beckers, T. (2013). Rating data are underrated: validity of US expectancy in human fear conditioning. Journal of Behavior Therapy and Experimental Psychiatry, 44(2), 201-206.

Boggiano, M., Dorsey, J., Thomas, J., \& Murdaugh, D. (2009). The Pavlovian power of palatable food: lessons for weight-loss adherence from a new rodent model of cue-induced overeating. International Journal of Obesity, 33(6), 693-701.

Bongers, P., Jansen, A., Havermans, R., Roefs, A., \& Nederkoorn, C. (2013). Happy eating: The underestimated role of overeating in a positive mood. Appetite, 67, 74-80.

Bongers, P., Jansen, A., Houben, K., \& Roefs, A. (2013). Happy Eating: The Single Target Implicit Association Test predicts overeating after positive emotions. Eating Behaviors, 14, 348-355.

Bongers, P., Van de Giessen, E., Roefs, A., Nederkoorn, C., Booij, J., Van den Brink, W., \& Jansen, A. (2015). Being Impulsive and Obese Increases Susceptibility to Speeded Detection of High-Calorie Foods. Health Psychology, $34,677-685$.

Bongers, P., Van den Akker, K., Havermans, R., \& Jansen, A. (2015). Emotional eating and Pavlovian learning: Does negative mood facilitate appetitive conditioning? Appetite, 89(0), 226-236.

Bongers, P., de Graaff, A., \& Jansen, A. (in press).'Emotional' does not even start to cover it: Generalization of overeating in emotional eaters. Appetite.

Boutelle, K. N., Zucker, N. L., Peterson, C. B., Rydell, S. A., Cafri, G., \& Harnack, L. (2011). Two novel treatments to reduce overeating in overweight children: a randomized controlled trial. Journal of Consulting and Clinical Psychology, 79(6), 759-771. 
Bouton, M. E. (1993). Context, time, and memory retrieval in the interference paradigms of Pavlovian learning. Psychological Bulletin, 114(1), 80-99.

Bouton, M. E. (2011). Learning and the persistence of appetite: Extinction and the motivation to eat and overeat. Physiology \& Behavior, 103(1), 51-58.

Bouton, M. E., Woods, A. M., Moody, E. W., Sunsay, C., \& García-Gutiérrez, A. (2006). Counteracting the ContextDependence of Extinction: Relapse and Tests of Some Relapse Prevention Methods. In M. G. Craske, D. Hermans, \& D. Vansteenwegen (Eds.), Fear and learning: From basic processes to clinical implications (pp. 175-196). Washington, DC: American Psychological Association.

Braden, A., Rhee, K., Peterson, C. B., Rydell, S. A., Zucker, N., \& Boutelle, K. (2014). Associations between child emotional eating and general parenting style, feeding practices, and parent psychopathology. Appetite, 80, 35-40.

Brogan, A., \& Hevey, D. (2013). Eating styles in the morbidly obese: restraint eating, but not emotional and external eating, predicts dietary behavior. Psychology \& Health, 28(6), 714-725.

Brondel, L., Romer, M., Van Wymelbeke, V., Pineau, N., Jiang, T., Hanus, C., et al. (2009). Variety enhances food intake in humans: role of sensory-specific satiety. Physiology \& Behavior, 97(1), 44-51.

Bruch, H. (1964). Psychological aspects of overeating and obesity. Psychosomatics, 5(5), 269-274.

Buckholtz, J. W., Treadway, M. T., Cowan, R. L., Woodward, N. D., Li, R., Ansari, M. S., et al. (2010). Dopaminergic network differences in human impulsivity. Science, 329(5991), 532-532.

Bulik, C. M., Lawson, R. H., \& Carter, F. A. (1996). Salivary reactivity in restrained and unrestrained eaters and women with bulimia nervosa. Appetite, 27(1), 15-24.

Carr, D., \& Friedman, M. A. (2005). Is obesity stigmatizing? Body weight, perceived discrimination, and psychological well-being in the United States. Journal of Health and Social Behavior, 46(3), 244-259.

Castellanos, E. H., Charboneau, E., Dietrich, M. S., Park, S., Bradley, B. P., Mogg, K., et al. (2009). Obese adults have visual attention bias for food cue images: evidence for altered reward system function. International Journal of Obesity, 33(9), 1063-1073.

Cepeda-Benito, A., Gleaves, D. H., Williams, T. L., \& Erath, S. A. (2000). The development and validation of the State and Trait Food-Cravings Questionnaires. Behavior Therapy, 31(1), 151-173.

Christensen, L., \& Brooks, A. (2006). Changing food preference as a function of mood. The journal of psychology, 140(4), 293-306.

Chua, J. L., Touyz, S., \& Hill, A. J. (2004). Negative mood-induced overeating in obese binge eaters: An experimental study. International Journal of Obesity, 28(4), 606-610.

Coletta, M., Platek, S., Mohamed, F. B., Van Steenburgh, J. J., Green, D., \& Lowe, M. R. (2009). Brain activation in restrained and unrestrained eaters: an fMRI study. Journal of Abnormal Psychology, 118(3), 598-609.

Conner, M., Fitter, M., \& Fletcher, W. (1999). Stress and snacking: A diary study of daily hassles and between-meal snacking. Psychology \& Health, 14(1), 51-63.

Cools, J., Schotte, D. E., \& McNally, R. J. (1992). Emotional arousal and overeating in restrained eaters. Journal of Abnormal Psychology, 101(2), 348-351.

Cooper, Z., Cooper, P. J., \& Fairburn, C. G. (1989). The validity of the eating disorder examination and its subscales. The British Journal of Psychiatry, 154(6), 807-812.

Cooper, Z., \& Fairburn, C. (1987). The eating disorder examination: A semi-structured interview for the assessment of the specific psychopathology of eating disorders. International Journal of Eating Disorders, 6(1), 1-8.

Corr, P. J. (2004). Reinforcement sensitivity theory and personality. Neuroscience \& Biobehavioral Reviews, 28(3), 317332.

Corr, P. J., Pickering, A. D., \& Gray, J. A. (1995). Personality and reinforcement in associative and instrumental learning. Personality and Individual Differences, 19(1), 47-71. 
Coskunpinar, A., \& Cyders, M. A. (2013). Impulsivity and substance-related attentional bias: A meta-analytic review. Drug and alcohol dependence, 133(1), 1-14.

Crawford, J. R., \& Henry, J.D. (2004). The Positive and Negative Affect Scedule (PANAS): Construct validity, measurement properties and normative data in a large non-clinical sample. British Journal of Clinical Psychology, $43(3), 245-265$

Dawe, S., \& Loxton, N. J. (2004). The role of impulsivity in the development of substance use and eating disorders. Neuroscience \& Biobehavioral Reviews, 28(3), 343-351.

De Houwer, J., Teige-Mocigemba, S., Spruyt, A., \& Moors, A. (2009). Implicit measures: A normative analysis and review. Psychological Bulletin, 135(3), 347-368.

De Weijer, B. A., Van de Giessen, E., Van Amelsvoort, T. A., Boot, E., Braak, B., Janssen, I. M., et al. (2011). Lower striatal dopamine D2/3 receptor availability in obese compared with non-obese subjects. EJNMMI research, 1(1), 1-5.

De Wit, H. (2009). Impulsivity as a determinant and consequence of drug use: a review of underlying processes. Addiction Biology, 14(1), 22-31.

Dingemans, A. E., Martijn, C., Jansen, A., \& Van Furth, E. F. (2009). The effect of suppressing negative emotions on eating behavior in binge eating disorder. Appetite, 52(1), 51-57.

Dingemans, A. E., Martijn, C., Van Furth, E. F., \& Jansen, A. (2009). Expectations, mood, and eating behavior in binge eating disorder. Beware of the bright side. Appetite, 53(2), 166-173.

Dols, M., Willems, B., Van Den Hout, M., \& Bittoun, R. (2000). Smokers can learn to influence their urge to smoke. Addictive Behaviors, 25(1), 103-108.

Doran, N., McChargue, D., \& Spring, B. (2008). Effect of impulsivity on cardiovascular and subjective reactivity to smoking cues. Addictive Behaviors, 33(1), 167-172.

Eldredge, K. L., \& Agras, W. S. (1996). Weight and shape overconcern and emotional eating in binge eating disorder. International Journal of Eating Disorders, 19(1), 73-82.

Enticott, P. G., Ogloff, J. R., \& Bradshaw, J. L. (2006). Associations between laboratory measures of executive inhibitory control and self-reported impulsivity. Personality and Individual Differences, 41(2), 285-294.

Epel, E., Lapidus, R., McEwen, B., \& Brownell, K. (2001). Stress may add bite to appetite in women: a laboratory study of stress-induced cortisol and eating behavior. Psychoneuroendocrinology, 26(1), 37-49.

Epstein, L. H., Paluch, R., \& Coleman, K. J. (1996). Differences in salivation to repeated food cues in obese and nonobese women. Psychosomatic Medicine, 58(2), 160-164.

Evers, C., Adriaanse, M., de Ridder, D. T., \& De Witt Huberts, J. C. (2013). Good mood food. Positive emotion as a neglected trigger for food intake. Appetite, 68, 1-7.

Evers, C., de Ridder, D. T. D., \& Adriaanse, M. A. (2009). Assessing yourself as an emotional eater: Mission impossible? Health Psychology, 28(6), 717-725.

Evers, C., Stok, F. M., \& de Ridder, D. T. (2010). Feeding your feelings: Emotion regulation strategies and emotional eating. Personality and Social Psychology Bulletin, 36, 792-804.

Fairburn, C. G. (2008). Cognitive Behavior Therapy and Eating Disorders. New York, NY: Guilford Press.

Fassino, S., Leombruni, P., Pierò, A., Daga, G. A., Amianto, F., Rovera, G., et al. (2002). Temperament and character in obese women with and without binge eating disorder. Comprehensive Psychiatry, 43(6), 431-437.

Ferriday, D., \& Brunstrom, J. (2011). 'I just can't help myself': effects of food-cue exposure in overweight and lean individuals. International Journal of Obesity, 35(1), 142-149.

Field, M., \& Cox, W. M. (2008). Attentional bias in addictive behaviors: a review of its development, causes, and consequences. Drug and Alcohol Dependence, 97(1), 1-20.

Galanti, K., Gluck, M. E., \& Geliebter, A. (2007). Test meal intake in obese binge eaters in relation to impulsivity and compulsivity. International Journal of Eating Disorders, 40(8), 727-732. 
Ganley, R. M. (1989). Emotion and eating in obesity: A review of the litreature. International Journal of Eating Disorders, 8(3), 343-361.

Geliebter, A., \& Aversa, A. (2003). Emotional eating in overweight, normal weight, and underweight individuals. Eating Behaviors, 3(4), 341-347.

Gerrards-Hesse, A., Spies, K., \& Hesse, F. W. (1994). Experimental inductions of emotional states and their effectiveness: A review. British Journal of Psychology, 85(1), 55-78.

Gibson, E. L. (2012). The psychobiology of comfort eating: implications for neuropharmacological interventions. Behavioral Pharmacology, 23, 442-460.

Greeno, C. G., \& Wing, R. R. (1994). Stress-induced eating. Psychological Bulletin, 115(3), 444-464.

Greenwald, A. G., McGhee, D. E., \& Schwartz, J. L. K. (1998). Measuring individual differences in implicit cognition: The implicit association test. Journal of Personality and Social Psychology, 74(6), 1464-1480.

Greenwald, A. G., Nosek, B. A., \& Banaji, M. R. (2003). Understanding and using the Implicit Association Test: An improved scoring algorithm. Journal of Personality and Social Psychology, 85(2), 197-216.

Groesz, L. M., McCoy, S., Carl, J., Saslow, L., Stewart, J., Adler, N., et al. (2012). What is eating you? Stress and the drive to eat. Appetite, 58(2), 717-721.

Grunberg, N. E., \& Straub, R. O. (1992). The role of gender and taste class in the effects of stress on eating. Health Psychology, 11(2), 97-100

Guerrieri, R., Nederkoorn, C., \& Jansen, A. (2007). How impulsiveness and variety influence food intake in a sample of healthy women. Appetite, 48(1), 119-122.

Guerrieri, R., Nederkoorn, C., \& Jansen, A. (2008). The effect of an impulsive personality on overeating and obesity: Current state of affairs. Psihologijske Teme, 17(2), 265-286.

Guerrieri, R., Nederkoorn, C., Schrooten, M., Martijn, C., \& Jansen, A. (2009). Inducing impulsivity leads high and low restrained eaters into overeating, whereas current dieters stick to their diet. Appetite, 53(1), 93-100.

Guerrieri, R., Nederkoorn, C., Stankiewicz, K., Alberts, H., Geschwind, N., Martijn, C., \& Jansen, A. (2007). The influence of trait and induced state impulsivity on food intake in normal-weight healthy women. Appetite, 49(1), 66-73.

Habhab, S., Sheldon, J. P., \& Loeb, R. C. (2009). The relationship between stress, dietary restraint, and food preferences in women. Appetite, 52(2), 437-444.

Haedt-Matt, A. A., \& Keel, P. K. (2011). Revisiting the affect regulation model of binge eating: a meta-analysis of studies using ecological momentary assessment. Psychological Bulletin, 137(4), 660.

Hay, P. (2013). A systematic review of evidence for psychological treatments in eating disorders: 2005-2012. International Journal of Eating Disorders, 46(5), 462-469.

Hays, N. P., \& Roberts, S. B. (2008). Aspects of eating behaviors 'disinhibition' and 'restraint' are related to weight gain and $\mathrm{BMI}$ in women. Obesity, 16(1), 52-58.

Health Monitor 2012 [Gezondheidsmonitor GGD'en, CBS en RIVM, 2012. In Dutch]. WWW Document (2013, September 12). Statistics Netherlands. Retrieved from http://www.cbs.nl/nl-NL/menu/themas/gezondheidwelzijn/cijfers/incidenteel/maatwerk/2013-gezondheidsmonitor2012-mw.htm. Accessed March 2015.

Heatherton, T. F., \& Baumeister, R. F. (1991). Binge eating as escape from self-awareness. Psychological Bulletin, 110(1), 86-108.

Heatherton, T. F., Herman, C. P., \& Polivy, J. (1991). Effects of physical threat and ego threat on eating behavior. Journal of Personality and Social Psychology, 60(1), 138-143.

Hendrick, O. M., Luo, X., Zhang, S., \& Li, C.-S. R. (2011). Saliency processing and obesity: a preliminary imaging study of the stop signal task. Obesity, 20(9), 1796-1802.

Hendy, H. M. (2012). Which comes first in food-mood relationships, foods or moods? Appetite, 58(2), 771-775. 
Hepworth, R., Mogg, K., Brignell, C., \& Bradley, B. P. (2010). Negative mood increases selective attention to food cues and subjective appetite. Appetite, 54(1), 134-142.

Herman, C. P., \& Mack, D. (1975). Restrained and unrestrained eating. Journal of Personality, 43(4), 647-660.

Herman, C. P., \& Polivy, J. (1980). Restrained eating. In A. J. Stunkard (Ed.), Obesity (pp. 208-225). Philadelphia: Saunders.

Hetherington, M. M., Foster, R., Newman, T., Anderson, A. S., \& Norton, G. (2006). Understanding variety: tasting different foods delays satiation. Physiology \& Behavior, 87(2), 263-271.

Hou, R., Mogg, K., Bradley, B. P., Moss-Morris, R., Peveler, R., \& Roefs, A. (2011). External eating, impulsivity and attentional bias to food cues. Appetite, 56, 424-427.

Jansen, A. (1990). Binge eating: notes and data. Doctoral dissertation. Maastricht University, The Netherlands. ISBN 9052910278 .

Jansen, A. (1998). A learning model of binge eating: Cue reactivity and cue exposure. Behavior Research and Therapy, 36, 257-272.

Jansen, A., Broekmate, J., \& Heymans, M. (1992). Cue-exposure vs self-control in the treatment of binge eating: a pilot study. Behavior Research and Therapy, 30(3), 235-241.

Jansen, A., Havermans, R. C., \& Nederkoorn, C. (2011). Cued overeating. V. R. Preedy, R. R. Watson, \& C. R. Martin (Eds.), Handbook of behavior, food and nutrition (pp. 1431-1443). New York, NY: Springer.

Jansen, A., Nederkoorn, C., Roefs, A., Bongers, P., Teugels, T., \& Havermans, R. (2011). The proof of the pudding is in the eating: Is the DEBQ - External Eating Scale a valid measure of external eating? International Journal of Eating Disorders, 44(2), 164-168.

Jansen, A., Nederkoorn, C., Van Baak, L., Keirse, C., Guerrieri, R., \& Havermans, R. (2009). High-restrained eaters only overeat when they are also impulsive. Behavior Research and Therapy, 47(2), 105-110.

Jansen, A., Theunissen, N., Slechten, K., Nederkoorn, C., Boon, B., Mulkens, S., \& Roefs, A. (2003). Overweight children overeat after exposure to food cues. Eating Behaviors, 4(2), 197-209.

Jansen, A., Van den Hout, M. A., De Loof, C., Zandbergen, J., \& Griez, E. (1989). A case of bulimia successfully treated by cue exposure. Journal of Behavior Therapy and Experimental Psychiatry, 20(4), 327-332.

Jansen, A., Vanreyten, A., Van Balveren, T., Roefs, A., Nederkoorn, C., \& Havermans, R. (2008). Negative affect and cue-induced overeating in non-eating disordered obesity. Appetite, 51(3), 556-562.

Johnson, F., Pratt, M., \& Wardle, J. (2012). Dietary restraint and self-regulation in eating behavior. International Journal of Obesity, 36(5), 665-674.

Kahan, D., Polivy, J., \& Herman, C. P. (2003). Conformity and dietary disinhibition: A test of the ego-strength model of self-regulation. International Journal of Eating Disorders, 33(2), 165-171.

Kandiah, J., Yake, M., Jones, J., \& Meyer, M. (2006). Stress influences appetite and comfort food preferences in college women. Nutrition Research, 26(3), 118-123.

Kaplan, H. I., \& Kaplan, H. S. (1957). The psychosomatic concept of obesity. Journal of Nervous and Mental Disease, 125, 181-201.

Karos, K., Meulders, A., \& Vlaeyen, J. W. (2014). Threatening Social Context Facilitates Pain-related Fear Learning. The Journal of Pain, 16, 214-225.

Karpinski, A., \& Steinman, R. B. (2006). The Single Category Implicit Association Test as a measure of implicit social cognition. Journal of Personality and Social Psychology, 91(1), 16-32.

Kemps, E., \& Tiggemann, M. (2009). Attentional bias for craving-related (chocolate) food cues. Experimental and Clinical Psychopharmacology, 17(6), 425-433.

Kenardy, J., Butler, A., Carter, C., \& Moor, S. (2003). Eating, mood, and gender in a noneating disorder population. Eating Behaviors, 4(2), 149-158. 
Koenders, P. G., \& Van Strien, T. (2011). Emotional eating, rather than lifestyle behavior, drives weight gain in a prospective study in 1562 employees. Journal of Occupational and Environmental Medicine, 53(11), 1287-1293.

Konttinen, H., Männistö, S., Sarlio-Lähteenkorva, S., Silventoinen, K., \& Haukkala, A. (2010). Emotional eating, depressive symptoms and self-reported food consumption. A population-based study. Appetite, 54 (3), 473-479.

Konttinen, H., Silventoinen, K., Sarlio-Lähteenkorva, S., Männistö, S., \& Haukkala, A. (2010). Emotional eating and physical activity self-efficacy as pathways in the association between depressive symptoms and adiposity indicators. The American Journal of Clinical Nutrition, 92(5), 1031-1039.

Kuijer, R. G., \& Boyce, J. A. (2012). Emotional eating and its effect on eating behavior after a natural disaster. Appetite, 58(3), 936-939.

Laitinen, J., Ek, E., \& Sovio, U. (2002). Stress-related eating and drinking behavior and body mass index and predictors of this behavior. Preventive Medicine, 34(1), 29-39.

Lang, P. J., Bradley, M. M., \& Cuthbert, B. N. (1999). International affective picture system (IAPS): Instruction manual and affective ratings. Gainesville, FL: The centre for research in psychophysiology, University of Florida.

Larsen, J. K., Hermans, R. C., \& Engels, R. C. (2012). Food intake in response to food-cue exposure. Examining the influence of duration of the cue exposure and trait impulsivity. Appetite, 58(3), 907-913.

Lattimore, P. (2001). Stress-induced eating: an alternative method for inducing ego-threatening stress. Appetite, 36(2), 187-188.

Lattimore, P., \& Caswell, N. (2004). Differential effects of active and passive stress on food intake in restrained and unrestrained eaters. Appetite, 42(2), 167-173.

Lattimore, P., \& Mead, B. R. (2015). See it, grab it, or STOP! Relationships between trait impulsivity, attentional bias for pictorial food cues and associated response inhibition following in-vivo food cue exposure. Appetite, 90, 248-253.

Logan, G. D., Schachar, R. J., \& Tannock, R. (1997). Impulsivity and inhibitory control. Psychological Science, 8(1), 60-64. Lovibond, P. F., \& Shanks, D. R. (2002). The role of awareness in Pavlovian conditioning: empirical evidence and theoretical implications. Journal of Experimental Psychology: Animal Behavior Processes, 28(1), 3-26.

Lowe, M. R., Annunziato, R. A., Markowitz, J.T., Didie, E., Bellace, D. L., Riddell, L., et al. (2006). Multiple types of dieting prospectively predict weight gain during the freshman year of college. Appetite, 47(1), 83-90.

Lowe, M. R., \& Fisher Jr, E. B. (1983). Emotional reactivity, emotional eating, and obesity: A naturalistic study. Journal of Behavioral Medicine, 6(2), 135-149.

Loxton, N. J., Dawe, S., \& Cahill, A. (2011). Does negative mood drive the urge to eat? The contribution of negative mood, exposure to food cues and eating style. Appetite, 56(2), 368-374.

Macht, M. (2008). How emotions affect eating: a five-way model. Appetite, 50(1), 1-11.

Macht, M., \& Dettmer, D. (2006). Everyday mood and emotions after eating a chocolate bar or an apple. Appetite, 46(3), 332-336.

Macht, M., Gerer, J., \& Ellgring, H. (2003). Emotions in overweight and normal-weight women immediately after eating foods differing in energy. Physiology \& Behavior, 80(2), 367-374.

Macht, M., \& Mueller, J. (2007). Immediate effects of chocolate on experimentally induced mood states. Appetite, $49(3), 667-674$

Macht, M., \& Simons, G. (2000). Emotions and eating in everyday life. Appetite, 35(1), 65-71

Marsh, D. M., Dougherty, D. M., Mathias, C. W., Moeller, F. G., \& Hicks, L. R. (2002). Comparisons of women with high and low trait impulsivity using behavioral models of response-disinhibition and reward-choice. Personality and Individual Differences, 33(8), 1291-1310.

Martinez-Mallén, E., Castro-Fornieles, J., Lázaro, L., Moreno, E., Morer, A., Font, E., et al. (2007). Cue exposure in the treatment of resistant adolescent bulimia nervosa. International Journal of Eating Disorders, 40(7), 596-601. 
Masheb, R. M., \& Grilo, C. M. (2006). Emotional overeating and its associations with eating disorder psychopathology among overweight patients with binge eating disorder. International Journal of Eating Disorders, 39(2), 141-146.

Mattes, R. D. (1997). Physiologic responses to sensory stimulation by food: nutritional implications. Journal of the American Dietetic Association, 97(4), 406-413.

Meyer, M. D., Risbrough, V. B., Liang, J., \& Boutelle, K. N. (2015). Pavlovian conditioning to hedonic food cues in overweight and lean individuals. Appetite, 87, 56-61.

Mikels, J. A., Fredrickson, B. L., Larkin, G. R., Lindberg, C. M., Maglio, S. J., \& Reuter-Lorenz, P. A. (2005). Emotional category data on images from the International Affective Picture System. Behavior Research Methods, 37(4), 626-630.

Milich, R. S. (1975). A critical analysis of Schachter's externality theory of obesity. Journal of Abnormal Psychology, 84(5), 586-588.

Mitterschiffthaler, M. T., Fu, C. H., Dalton, J. A., Andrew, C. M., \&Williams, S. C. (2007). A functional MRI study of happy and sad affective states induced by classical music. Human Brain Mapping, 28(11), 1150-1162.

Mobbs, O., Crépin, C., Thiéry, C., Golay, A., \& Van der Linden, M. (2010). Obesity and the four facets of impulsivity. Patient Education and Counseling, 79(3), 372-377.

Myerson, J., Green, L., \& Warusawitharana, M. (2001). Area under the curve as a measure of discounting. Journal of the Experimental Analysis of Behavior, 76(2), 235-243.

Nederkoorn, C., Smulders, F., \& Jansen, A. (1999). Recording of swallowing events using electromyography as a non-invasive measurement of salivation. Appetite, 33(3), 361-369.

Nederkoorn, C., Smulders, F. T., Havermans, R. C., Roefs, A., \& Jansen, A. (2006). Impulsivity in obese women. Appetite, $47(2), 253-256$.

Nederkoorn, C., Smulders, F. T. Y., \& Jansen, A. (2000). Cephalic phase responses, craving and food intake in normal subjects. Appetite, 35(1), 45-55.

Newman, E., O'Connor, D. B., \& Conner, M. (2007). Daily hassles and eating behavior: the role of cortisol reactivity status. Psychoneuroendocrinology, 32(2), 125-132.

Nguyen-Rodriguez, S. T., Chou, C.-P., Unger, J. B., \& Spruijt-Metz, D. (2008). BMl as a moderator of perceived stress and emotional eating in adolescents. Eating Behaviors, 9(2), 238-246.

Nijs, I. M., Muris, P., Euser, A. S., \& Franken, I. H. (2010). Differences in attention to food and food intake between overweight/obese and normal-weight females under conditions of hunger and satiety. Appetite, 54(2), $243-254$.

Nijs, I. M. T., Franken, I. H. A., \& Muris, P. (2007). The modified Trait and State Food-Cravings Questionnaires: Development and validation of a general index of food craving. Appetite, 49(1), 38-46.

Nisbett, R. E. (1968). Taste, deprivation, and weight determinants of eating behavior. Journal of Personality and Social Psychology, 10(2), 107-116.

Nolan, L. J., Halperin, L. B., \& Geliebter, A. (2010). Emotional Appetite Questionnaire. Construct validity and relationship with BMI. Appetite, 54(2), 314-319.

Norton, G. N. M., Anderson, A. S., \& Hetherington, M. M. (2006). Volume and variety: Relative effects on food intake. Physiology \& Behavior, 87, 714-722.

Nummenmaa, L., Hietanen, J. K., Calvo, M. G., \& Hyönä, J. (2011). Food catches the eye but not for everyone: a $\mathrm{BMl}$-contingent attentional bias in rapid detection of nutriments. PloS One, 6(5), e19215.

O'Connor, D. B., Jones, F., Conner, M., McMillan, B., \& Ferguson, E. (2008). Effects of daily hassles and eating style on eating behavior. Health Psychology, 27(1, Suppl), S20-S31.

O'Connor, D. B., \& O'Connor, R. C. (2004). Perceived changes in food intake in response to stress: The role of conscientiousness. Stress and Health, 20(5), 279-291. 
Ogden, C. L., Carroll, M. D., Kit, B. K., \& Flegal, K. M. (2014). Prevalence of childhood and adult obesity in the United States, 2011-2012. The Journal of the American Medical Association, 311(8), 806-814.

Oliver, G., Wardle, J., \& Gibson, L. (2000). Stress and food choice: A laboratory study. Psychosomatic Medicine, 62(6), 853-865.

Ouwens, M. A., Van Strien, T., \& Van Leeuwe, J. F. (2009). Possible pathways between depression, emotional and external eating. A structural equation model. Appetite, 53(2), 245-248.

Paisey, T. J., \& Mangan, G. L. (1988). Personality and conditioning with appetitive and aversive stimuli. Personality and Individual Differences, 9(1), 69-78.

Papachristou, H., Nederkoorn, C., Beunen, S., \& Jansen, A. (2013). Dissection of appetitive conditioning: Does impulsivity play a role? Appetite, 69, 46-53.

Papachristou, H., Nederkoorn, C., Havermans, R., Van der Horst, M., \& Jansen, A. (2012). Can't stop the craving: The effect of impulsivity on cue-elicited craving for alcohol in heavy and light social drinkers. Psychopharmaco/ogy, 219(2), 511-518.

Patel, K. A., \& Schlundt, D. G. (2001). Impact of moods and social context on eating behavior. Appetite, 36(2), 111-118.

Patton, J. H., Stanford, M. S., \& Barratt, E. S. (1995). Factor structure of the Barratt impulsiveness scale. Journal of Clinical Psychology, 51(6), 768-774.

Peeters, F. P. M. L., Ponds, R. W. H. M., \& Vermeeren, M. T. G. (1996). Affectiviteit en zelfbeoordeling van depressie en angst [Affectivity and self-report of depression and anxiety]. Tijdschrift voor Psychiatrie, 38, 240-250.

Péneau, S., Ménard, E., Méjean, C., Bellisle, F., \& Hercberg, S. (2013). Sex and dieting modify the association between emotional eating and weight status. The American Journal of Clinical Nutrition, 97(6), 1307-1313.

Pinaquy, S., Chabrol, H., Simon, C., Louvet, J. P., \& Barbe, P. (2003). Emotional Eating, Alexithymia, and Binge-Eating Disorder in Obese Women. Obesity Research, 11(2), 195-201.

Polivy, J., \& Herman, C. P. (2002). Causes of eating disorders. Annual Review of Psychology, 53(1), 187-213.

Puhl, R. M., \& Heuer, C. A. (2009). The stigma of obesity: a review and update. Obesity, 17(5), 941-964.

Pursey, K. M., Stanwell, P., Callister, R. J., Brain, K., Collins, C. E., \& Burrows, T. L. (2014). Neural responses to visual food cues according to weight status: A systematic review of functional magnetic resonance imaging studies. Frontiers in Nutrition, 1, 7.

Ransley, J., Donnelly, J., Botham, H., Khara, T., Greenwood, D., \& Cade, J. (2003). Use of supermarket receipts to estimate energy and fat content of food purchased by lean and overweight families. Appetite, 41(2), 141-148.

Raspopow, K., Abizaid, A., Matheson, K., \& Anisman, H. (2014). Anticipation of a psychosocial stressor differentially influences ghrelin, cortisol and food intake among emotional and non-emotional eaters. Appetite, 74, 35-43.

Reynolds, B., Ortengren, A., Richards, J. B., \& De Wit, H. (2006). Dimensions of impulsive behavior: Personality and behavioral measures. Personality and Individual Differences, 40(2), 305-315.

Ricca, V., Castellini, G., Fioravanti, G., Sauro, C. L., Rotella, F., Ravaldi, C., et al. (2012). Emotional eating in anorexia nervosa and bulimia nervosa. Comprehensive Psychiatry, 53(3), 245-251.

Rodgers, R. F., Paxton, S. J., Massey, R., Campbell, K. J., Wertheim, E. H., Skouteris, H., et al. (2013). Maternal feeding practices predict weight gain and obesogenic eating behaviors in young children: a prospective study. International Journal of Behavioral Nutrition and Physical Activity, 10(1), 24-33.

Rohleder, N., Wolf, J. M., Maldonado, E. F., \& Kirschbaum, C. (2006). The psychosocial stress-induced increase in salivary alpha-amylase is independent of saliva flow rate. Psychophysiology, 43(6), 645-652.

Rosen, J. C., Vara, L., Wendt, S., \& Leitenberg, H. (1990). Validity studies of the eating disorder examination. International Journal of Eating Disorders, 9(5), 519-528.

Rydén, A., Sullivan, M., Torgerson, J. S., Karlsson, J., Lindroos, A. K., \& Taft, C. (2003). Severe obesity and personality: a comparative controlled study of personality traits. International Journal of Obesity, 27, 1534-1540. 
Schachter, S. (1968). Obesity and eating. Science, 161, 751-756.

Schachter, S., Goldman, R., \& Gordon, A. (1968). Effects of fear, food deprivation, and obesity on eating. Journal of Personality and Social Psychology, 10(2), 91-97.

Schneider, K. L., Appelhans, B. M., Whited, M. C., Oleski, J., \& Pagoto, S. L. (2010). Trait anxiety, but not trait anger, predisposes obese individuals to emotional eating. Appetite, 55(3), 701-706.

Schneider, K. L., Panza, E., Appelhans, B. M., Whited, M. C., Oleski, J. L., \& Pagoto, S. L. (2012). The emotional eating scale. Can a self-report measure predict observed emotional eating? Appetite, 58(2), 563-566.

Schoenmakers, T. M., de Bruin, M., Lux, I. F., Goertz, A. G., Van Kerkhof, D. H., \& Wiers, R. W. (2010). Clinical effectiveness of attentional bias modification training in abstinent alcoholic patients. Drug and Alcohol Dependence, 109(1), 30-36.

Schoenmakers, T. M., Wiers, R. W., Jones, B. T., Bruce, G., \& Jansen, A. (2007). Attentional re-training decreases attentional bias in heavy drinkers without generalization. Addiction, 102(3), 399-405.

Schokker, D., Visscher, T., Nooyens, A., Van Baak, M., \& Seidell, J. (2007). Prevalence of overweight and obesity in the Netherlands. Obesity Reviews, 8(2), 101-107.

Schyns, G., Roefs, A., Mulkens, S., \& Jansen, A. (submitted). Expectancy violation, reduction of food cue reactivity and less eating in the absence of hunger after one food cue exposure session for overweight and obese women. Manuscript submitted for publication.

Sellahewa, D. A., \& Mullan, B. (2015). Health behaviors and their facilitation under depletion conditions: The case of snacking. Appetite, 90(0), 194-199.

Sheppard-Sawyer, C. L., McNally, R. J., \& Fischer, J. H. (2000). Film-induced sadness as a trigger for disinhibited eating. International Journal of Eating Disorders, 28(2), 215-220.

Small, D. M., Zatorre, R. J., Dagher, A., Evans, A. C., \& Jones-Gotman, M. (2001). Changes in brain activity related to eating chocolate from pleasure to aversion. Brain, 124(9), 1720-1733.

Smeets, E., Roefs, A., \& Jansen, A. (2009). Experimentally induced chocolate craving leads to an attentional bias in increased distraction but not in speeded detection. Appetite, 53(3), 370-375.

Smeets, E., Roefs, A., Van Furth, E., \& Jansen, A. (2008). Attentional bias for body and food in eating disorders: Increased distraction, speeded detection, or both? Behavior Research and Therapy, 46(2), 229-238.

Snoek, H. M., Engels, R. C. M. E., Janssens, J. M. A. M., \& Van Strien, T. (2007). Parental behavior and adolescents' emotional eating. Appetite, 49(1), 223-230.

Sobik, L., Hutchison, K., \& Craighead, L. (2005). Cue-elicited craving for food: a fresh approach to the study of binge eating. Appetite, 44(3), 253-261.

Solomon, M. R. (2001). Eating as both coping and stressor in overweight control. Journal of Advanced Nursing, 36(4), 563-572.

Stanford, M. S., Mathias, C. W., Dougherty, D. M., Lake, S. L., Anderson, N. E., \& Patton, J. H. (2009). Fifty years of the Barratt Impulsiveness Scale: An update and review. Personality and Individual Differences, 47(5), 385-395.

Stegen, K., De Bruyne, K., Rasschaert, W., Van de Woestijne, K. P., \&Van den Bergh, O. (1999). Fear-relevant images as conditioned stimuli for somatic complaints, respiratory behavior, and reduced end-tidal $\mathrm{pCO}_{2}$. Journal of Abnormal Psychology, 108(1), 143-152.

Stein, C. J., \& Colditz, G. A. (2004). The Epidemic of Obesity. The Journal of Clinical Endocrinology \& Metabolism, 89(6), 2522-2525.

Stice, E., Spoor, S., Bohon, C., Veldhuizen, M. G., \& Small, D. M. (2008). Relation of reward from food intake and anticipated food intake to obesity: a functional magnetic resonance imaging study. Journal of Abnormal Psychology, 117(4), 924-935. 
Stoeckel, L. E., Weller, R. E., Cook III, E. W., Twieg, D. B., Knowlton, R. C., \& Cox, J. E. (2008). Widespread reward-system activation in obese women in response to pictures of high-calorie foods. Neuroimage, 41(2), 636-647.

Stunkard, A. J., \& Messick, S. (1985). The three-factor eating questionnaire to measure dietary restraint, disinhibition and hunger. Journal of Psychosomatic Research, 29(1), 71-83.

Svaldi, J., Caffier, D., \& Tuschen-Caffier, B. (2010). Emotion suppression but not reappraisal increases desire to binge in women with binge eating disorder. Psychotherapy and Psychosomatics, 79(3), 188-190.

Tabachnick, B. G., Fidell, L. S., \& Osterlind, S. J. (2001). Using multivariate statistics. Boston, MA: Allyn and Bacon.

Telch, C. F., \& Agras, W. S. (1996). Do emotional states influence binge eating in the obese? International Journal of Eating Disorders, 20(3), 271-279.

Tetley, A., Brunstrom, J., \& Griffiths, P. (2009). Individual differences in food-cue reactivity. The role of BMI and everyday portion-size selections. Appetite, 52(3), 614-620.

Tetley, A. C., Brunstrom, J. M., \& Griffiths, P. L. (2010). The role of sensitivity to reward and impulsivity in food-cue reactivity. Eating Behaviors, 11(3), 138-143.

Thewissen, R., Havermans, R. C., Geschwind, N., Van den Hout, M., \& Jansen, A. (2007). Pavlovian conditioning of an approach bias in low-dependent smokers. Psychopharmacology, 194(1), 33-39.

Thewissen, R., Van Den Hout, M., Havermans, R. C., \& Jansen, A. (2005). Context-dependency of cue-elicited urge to smoke. Addiction, 100(3), 387-396.

Topham, G. L., Hubbs-Tait, L., Rutledge, J. M., Page, M. C., Kennedy, T. S., Shriver, L. H., et al. (2011). Parenting styles, parental response to child emotion, and family emotional responsiveness are related to child emotional eating. Appetite, 56(2), 261-264.

Toro, J., Cervera, M., Feliu, M., Garriga, N., Jou, M., Martinez, E., et al. (2003). Cue exposure in the treatment of resistant bulimia nervosa. International Journal of Eating Disorders, 34(2), 227-234.

Tuomisto, T., Tuomisto, M. T., Hetherington, M., \& Lappalainen, R. (1998). Reasons for initiation and cessation of eating in obese men and women and the affective consequences of eating in everyday situations. Appetite, 30(2), 211-222.

Turner, S. A., Luszczynska, A., Warner, L., \& Schwarzer, R. (2010). Emotional and uncontrolled eating styles and chocolate chip cookie consumption. A controlled trial of the effects of positive mood enhancement. Appetite, 54(1), 143-149.

Udo, T., Grilo, C. M., Brownell, K. D., Weinberger, A. H., DiLeone, R. J., \& McKee, S. A. (2013). Modeling the effects of positive and negative mood on the ability to resist eating in obese and non-obese individuals. Eating Behaviors, 14(1), 40-46.

Vainik, U., Neseliler, S., Konstabel, K., Fellows, L. K., \& Dagher, A. (2015). Eating traits questionnaires as a continuum of a single concept. Uncontrolled eating. Appetite, 90, 229-239.

Van de Giessen, E., Celik, F., Schweitzer, D. H., Van den Brink, W., \& Booij, J. (2014). Dopamine D2/3 receptor availability and amphetamine-induced dopamine release in obesity. Journal of Psychopharmacology, 1-8.

Van den Akker, K., Havermans, R. C., Bouton, M. E., \& Jansen, A. (2014). How partial reinforcement of food cues affects the extinction and reacquisition of appetitive responses. A new model for dieting success? Appetite, 81, 242-252.

Van den Akker, K., Havermans, R. C., \& Jansen, A. (2015). Effects of occasional reinforced trials during extinction on the reacquisition of conditioned responses to food cues. Journal of Behavior Therapy and Experimental Psychiatry, 48, 50-58.

Van den Akker, K., Jansen, A., Frentz, F., \& Havermans, R. C. (2013). Impulsivity makes more susceptible to overeating after contextual appetitive conditioning. Appetite, 70, 73-80. 
Van den Akker, K., Stewart, K., Antoniou, E. E., Palmberg, A., \& Jansen, A. (2014). Food Cue Reactivity, Obesity, and Impulsivity: Are They Associated? Current Addiction Reports, 1(4), 301-308.

Van Den Bergh, O., Kempynck, P. J., Van De Woestijne, K. P., Baeyens, F., \& Eelen, P. (1995). Respiratory learning and somatic complaints: a conditioning approach using $\mathrm{CO}$ 2-enriched air inhalation. Behavior Research and Therapy, 33(5), 517-527.

Van den Bergh, O., Stegen, K., \& Van de Woestijne, K. P. (1997). Learning to have psychosomatic complaints: conditioning of respiratory behavior and somatic complaints in psychosomatic patients. Psychosomatic Medicine, 59(1), 13-23.

Van den Bergh, O., Stegen, K., \&Van de Woestijne, K. P. (1998). Memory effects on symptom reporting in a respiratory learning paradigm. Health Psychology, 17(3), 241-248.

Van der Does, A. J.W. (2002). Handleiding bij de Nederlandse versie van Beck Depression Inventory-second edition (BDI-II-NL) [Manual for the Dutch version of the Beck Depression Inventory-second edition (BDI-II-NL)]. Harcourt Assessment, The Netherlands.

Van Gucht, D., Baeyens, F., Hermans, D., \& Beckers, T. (2013). The inertia of conditioned craving. Does context modulate the effect of counterconditioning? Appetite, 65, 51-57.

Van Gucht, D., Baeyens, F., Vansteenwegen, D., Hermans, D., \& Beckers, T. (2010). Counterconditioning reduces cueinduced craving and actual cue-elicited consumption. Emotion, 10(5), 688-695.

Van Gucht, D., Vansteenwegen, D., Beckers, T., \& Van Den Bergh, O. (2008). Return of experimentally induced chocolate craving after extinction in a different context: Divergence between craving for and expecting to eat chocolate. Behavior Research and Therapy, 46(3), 375-391.

Van Gucht, D., Vansteenwegen, D., Van den Bergh, O., \& Beckers, T. (2008). Conditioned craving cues elicit an automatic approach tendency. Behavior Research and Therapy, 46(10), 1160-1169.

Van Strien, T. (2005). Nederlandse Vragenlijst voor Eetgedrag 2005. Handleiding en Verantwoording [Manual of the Dutch Eating Behavior Questionnaire 2005]: Amsterdam: Boom test uitgevers.

Van Strien, T., Cebolla, A., Etchemendy, E., Gutiérrez-Maldonado, J., Ferrer-García, M., Botella, C., et al. (2013). Emotional eating and food intake after sadness and joy. Appetite, 66, 20-25.

Van Strien, T., Frijters, J. E., Bergers, G. P., \& Defares, P. B. (1986). The Dutch Eating Behavior Questionnaire (DEBQ) for assessment of restrained, emotional, and external eating behavior. International Journal of Eating Disorders, 5(2), 295-315.

Van Strien, T., Frijters, J. E. R., Van Staveren, W. A., Defares, P. B., \& Deurenberg, P. (1986). The predictive validity of the Dutch restrained eating scale. International Journal of Eating Disorders, 5(4), 747-755.

Van Strien, T., Herman, C. P., Anschutz, D. J., Engels, R. C. M. E., \& de Weerth, C. (2012). Moderation of distress-induced eating by emotional eating scores. Appetite, 58(1), 277-284.

Van Strien, T., Herman, C. P., \& Verheijden, M. W. (2009). Eating style, overeating, and overweight in a representative Dutch sample. Does external eating play a role? Appetite, 52(2), 380-387.

Van Strien, T., \& Ouwens, M. A. (2003). Counterregulation in female obese emotional eaters: Schachter, Goldman, and Gordon's (1968) test of psychosomatic theory revisited. Eating Behaviors, 3(4), 329-340.

Van Strien, T., Ouwens, M. A., Engel, C., \& de Weerth, C. (2014). Hunger, inhibitory control and distress-induced emotional eating. Appetite, 79(0), 124-133.

Van Strien, T., Roelofs, K., \& de Weerth, C. (2013). Cortisol reactivity and distress-induced emotional eating. Psychoneuroendocrinology, 38(5), 677-684.

Velázquez-Sánchez, C., Ferragud, A., Moore, C. F., Everitt, B. J., Sabino, V., \& Cottone, P. (2014). High Trait Impulsivity Predicts Food Addiction-Like Behavior in the Rat. Neuropsychopharmacology, 39, 2463-2472. 
References

Vohs, K. D., \& Heatherton, T. F. (2000). Self-regulatory failure: A resource-depletion approach. Psychological Science, $11(3), 249-254$.

Vuoskoski, J. K., \& Eerola, T. (2012). Can sad music really make you sad? Indirect measures of affective states induced by music and autobiographical memories. Psychology of Aesthetics, Creativity, and the Arts, 6(3), 204-213.

Wallis, D. J., \& Hetherington, M. M. (2004). Stress and eating: The effects of ego-threat and cognitive demand on food intake in restrained and emotional eaters. Appetite, 43(1), 39-46.

Wang, G.-J., Volkow, N. D., Logan, J., Pappas, N. R., Wong, C. T., Zhu, W., et al. (2001). Brain dopamine and obesity. The Lancet, 357(9253), 354-357.

Wansink, B., Cheney, M. M., \& Chan, N. (2003). Exploring comfort food preferences across age and gender. Physiology \& Behavior, 79(4), 739-747.

Wardle, J. (1990). Conditioning processes and cue exposure in the modification of excessive eating. Addictive Behaviors, 15(4), 387-393.

Watson, D., Clark, L. A., \& Tellegen, A. (1988). Development and validation of brief measures of positive and negative affect: The PANAS scales. Journal of Personality and Social Psychology, 54(6), 1063-1070.

Weingarten, H.P. (1983). Conditioned cues elicit feeding in sated rats: A role for learning in meal initiation. Science, 220(4595), 431-433.

Weinstein, S. E., Shide, D. J., \& Rolls, B. J. (1997). Changes in food intake in response to stress in men and women: psychological factors. Appetite, 28(1), 7-18.

Weller, R. E., Cook, E. W., Avsar, K. B., \& Cox, J. E. (2008). Obese women show greater delay discounting than healthyweight women. Appetite, 51(3), 563-569.

Werthmann, J., Renner, F., Roefs, A., Huibers, M. J. H., Plumanns, L., Krott, N., et al. (2014). Looking at food in sad mood: Do attention biases lead emotional eaters into overeating after a negative mood induction? Eating Behaviors, 15(2), 230-236

Werthmann, J., Roefs, A., Nederkoorn, C., Mogg, K., Bradley, B. P., \& Jansen, A. (2011). Can(not) take my eyes off it: Attention bias for food in overweight participants. Health Psychology, 30(5), 561-569.

Westenhoefer, J. (1991). Dietary restraint and disinhibition: is restraint a homogeneous construct? Appetite, 16(1), 45-55.

Whisman, M. A., Perez, J. E., \& Ramel, W. (2000). Factor structure of the Beck Depression Inventory-Second Edition (BDI-II) in a student sample. Journal of Clinical Psychology, 56, 545-551.

Whiteside, S. P., \& Lynam, D. R. (2001). The five factor model and impulsivity: Using a structural model of personality to understand impulsivity. Personality and Individual Differences, 30(4), 669-689.

Wiers, R. W., Eberl, C., Rinck, M., Becker, E. S., \& Lindenmeyer, J. (2011). Retraining automatic action tendencies changes alcoholic patients' approach bias for alcohol and improves treatment outcome. Psychological Science, 22(4), 490-497.

Wilson, G. T., Grilo, C. M., \& Vitousek, K. M. (2007). Psychological treatment of eating disorders. American Psychologist, 62(3), 199-216

Wyatt, S. B., Winters, K. P., \& Dubbert, P. M. (2006). Overweight and obesity: prevalence, consequences, and causes of a growing public health problem. The American Journal of the Medical Sciences, 331(4), 166-174.

Yeomans, M. R., \& Coughlan, E. (2009). Mood-induced eating. Interactive effects of restraint and tendency to overeat. Appetite, 52(2), 290-298.

Yeomans, M. R., Leitch, M., \& Mobini, S. (2008). Impulsivity is associated with the disinhibition but not restraint factor from the Three Factor Eating Questionnaire. Appetite, 50(2), 469-476.

Zellner, D. A., Loaiza, S., Gonzalez, Z., Pita, J., Morales, J., Pecora, D., et al. (2006). Food selection changes under stress. Physiology \& Behavior, 87(4), 789-793. 
Zinbarg, R., \& Mohlman, J. (1998). Individual differences in the acquisition of affectively valenced associations. Journal of Personality and Social Psychology, 74(4), 1024-1040.

Zinbarg, R., \& Revelle, W. (1989). Personality and conditioning: A test of four models. Journal of Personality and Social Psychology, 57(2), 301-314. 

Summary 

Considering the ever-growing obesity epidemic, it is of high importance to investigate any factor that can contribute to overeating and weight gain. One such factor, and the topic of this dissertation, is emotional eating. In its original definition, emotional eating refers to overeating in response to negative emotions. The main focus of this dissertation is on the specific cues that elicit overeating in emotional eaters, and on the role that classical conditioning plays in emotional eating. Attention is also given to the influence of individual differences on cued responding.

Chapter 1 provides a background on emotional eating. The chapter starts with the current state of affairs in emotional eating research by giving an extensive overview of previous studies on the topic. This is followed by an account of theories that have attempted to explain emotional eating. Classical conditioning is then introduced as an important mechanism. The chapter ends with an outline of the dissertation.

\section{Cue-elicited overeating in emotional eaters}

Chapters $\mathbf{2}$ and $\mathbf{3}$ both describe studies that aimed to investigate the role of positive emotions in emotional eating. Similar in design, participants in both studies underwent a positive, negative, or neutral mood induction, which was achieved by showing them 3-minute movie excerpts. In the first study, participants' food intake was measured during a bogus taste test, consisting of crisps and chocolate, after the mood induction. In the second study, milkshake intake was measured during the mood induction, as well as after. Furthermore, in the first study only a self-report measure of emotional eating was used, while an implicit measure (i.e., single-target IAT) was added in the second study. With regard to the self-report measure, the results of the two studies were partially in agreement. Both studies revealed 
an absence of increased food intake after negative emotions. With regard to positive emotions, in the first study emotional eaters consumed more food in the positive compared to the neutral condition, and showed higher intake after positive emotions compared to non-emotional eaters. In the second study, no increased intake in response to positive emotions was observed. In line with the self-report data of the first study, the IAT-data showed increased food intake in emotional eaters when feeling positive, but not when feeling negative. Again, no higher food consumption was observed in the negative mood. Chapter 2 also assessed changes in mood after consumption of palatable food. It was found that for both emotional and non-emotional eaters, mood increased after 5 minutes of eating and stayed at this level during the remaining 10 minutes of the taste test. Taken together, these studies suggest that positive emotions should not be underestimated in emotional eating. Positive emotions led to higher food intake than negative emotions in emotional eaters.

Chapter 4 reports on an additional study, in which it was hypothesized that emotional eaters overeat in response to a variety of cues, not restricted to merely negative emotions. Participants took part in four conditions (negative mood, positive mood, food exposure and control) divided over two sessions. Each manipulation was followed by a bogus taste test. Results were largely in support of the hypothesis. Emotional eaters identified on basis of self-report or on actual food intake in a negative mood consumed more food than non-emotional eaters in all experimental conditions, as well as the control condition. Furthermore, intake in emotional eaters did not differ between the experimental and control conditions. The data indicate that 'emotional eating' does not adequately describe the eating behaviour of so-called emotional eaters. It is proposed that emotional eating might therefore be more appropriately termed 'cue-reactive eating'.

\section{Individual differences: trait food craving, impulsivity, and weight status}

Chapter $\mathbf{5}$ shows a study designed to elucidate the role of trait craving, impulsivity and weight status in cue reactivity, more specifically attention bias. Obese and healthy-weight adults were measured on self-reported trait craving and trait impulsivity, as well as on two behavioural measures of impulsivity. They then participated in a visual search task, intended to assess speeded detection of and increased distraction by high- and low-caloric foods. Significant effects emerged with regard to self-reported trait impulsivity: Obese impulsive participants showed speeded detection of high-caloric foods compared to healthy-weight impulsive participants. No effects were found on increased distraction. Obese and healthyweight individuals scoring low on impulsivity differed on neither type of attention bias. 


\section{The role of classical conditioning in emotional eating}

Chapters $\mathbf{6}$ and $\mathbf{7}$ focus on two conditioning pathways that are proposed to be potentially involved in emotional eating, namely 1) facilitated conditioning in a negative mood and 2) development of negative emotional states into conditioned stimuli. In chapter 6 it was investigated whether a negative mood facilitates appetitive conditioning to neutral stimuli, and whether this is influenced by trait impulsivity. Participants who were brought into a negative or a neutral mood underwent a conditioning procedure, in which one neutral object (i.e., a vase with flowers) was paired with chocolate mousse intake, while a similar object was not. During and after the procedure expectancy and desire to eat, salivation and food intake were measured. Results showed successful appetitive conditioning in terms of expectancy and desire to eat, but no differences between the two conditions. A negative mood did not facilitate appetitive conditioning. An effect of impulsivity was only apparent in the taste test, in which high impulsive participants consumed less food when presented with the CS+ (i.e., vase that was paired with chocolate) compared to the CS- (i.e., vase that was not paired with chocolate).

Chapter 7 reports on the other pathway, in which negative emotions were hypothesized to develop into conditioned stimuli and predictors of food intake. Participants underwent a conditioning procedure in which they were presented with 10 different stimuli, five of which induced negative feelings, and five which induced neutral feelings. Participants were randomly divided over two conditions, one in which negative feelings were followed by eating chocolate but neutral feelings were not (FoodNeg condition), and one in which this was the other way around (FoodNeu condition). Expectancy and desire to eat were measured, as well as salivation, unpleasantness of experiencing negative emotions, and a chocolate vs. money choice task was included. The expectancy and desire data showed that negative emotions can indeed develop into conditioned stimuli. Participants who received chocolate after negative feelings reported increased expectancy and desire to eat when feeling negative compared to feeling neutral. A stronger preference for chocolate over food in a negative mood was observed for participants in the FoodNeg condition, but only at high levels of BMI. Furthermore, repeated exposure to negative feelings did not affect unpleasantness of experiencing such feelings in low emotional eaters, but did increase unpleasantness in high emotional eaters. However, when negative emotions were paired with chocolate intake, this effect was no longer apparent, and there was even a decrease in experienced unpleasantness. Thus, in emotional eaters, consumption of palatable food during a negative emotional experience seems to mitigate the effects of that experience. 
Based on the studies' results, it seems likely that classical conditioning processes can lead to emotional eating.

Chapter $\mathbf{8}$ presents a discussion and conclusion regarding each of the three focus points as outlined at the start of this summary. The first main conclusion is that emotional eaters do not merely overeat in response to negative emotions, but to do so after other cues as well. Second, classical conditioning is likely to play a role in emotional eating. Third, individual differences can influence cued responding and learning. Suggestions for future research based on the current findings are made. In addition, methodological considerations concerning the studies are given, and clinical implications of the findings are addressed. 


Samenvatting 

Gezien de nog altijd groeiende obesitas-epidemie is het van groot belang om de factoren die bijdragen aan overeten en gewichtstoename te onderzoeken. Eén van deze factoren, en het onderwerp van dit proefschrift, is emotioneel eten. In zijn oorspronkelijke definitie verwijst emotioneel eten naar overeten als reactie op het ervaren van negatieve emoties, maar onderzoek heeft laten zien dat deze omschrijving het gedrag wellicht niet geheel dekt. In dit proefschrift ligt de nadruk daarom ten eerste op de specifieke cues (interne of externe gebeurtenissen; inclusief - maar niet beperkt tot - negatieve emoties) die overeten in emotionele eters uitlokken. Ook wordt aandacht besteed aan de invloed van individuele verschillen (zoals impulsiviteit of gewicht) op hoe mensen op zulke eet-cues reageren (cue reactiviteit). Ten tweede richt dit proefschrift zich op de rol die klassieke conditionering speelt in emotioneel eten. Klassieke conditionering is een vorm van leren. In het geval van eten ontwikkelt een voorheen neutrale stimulus zich tot een voorspeller van eten (dit heet ook wel appetitieve conditionering). Dat gebeurt als die neutrale stimulus herhaaldelijk met eten gepaard gaat. Na een tijd is enkel het opmerken van de neutrale stimulus al genoeg om zin te krijgen in eten. lemand die elke week chips eet tijdens het kijken van zijn favoriete tv-programma, en op die manier chips en het programma met elkaar associeert, kan na een tijdje bij alleen het horen van de begintune van het programma al zin in chips krijgen. Als bekend is bij welke cues emotionele eters zich overeten en hoe emotioneel eten ontstaat, kan die kennis tijdens de behandeling van overeten gebruikt worden om dat gedrag te stoppen of te voorkomen.

Hoofdstuk 1 geeft achtergrondinformatie over emotioneel eten. Het hoofdstuk begint met een uitgebreid overzicht van eerdere studies, waarmee de huidige stand van zaken in onderzoek naar emotioneel eten wordt geschetst. Daarna volgt een bespreking van 
theorieën die getracht hebben emotioneel eten te verklaren, en wordt klassieke conditionering naar voren gebracht als een belangrijk mechanisme. Het hoofdstuk eindigt met een weergave van de structuur van dit proefschrift.

\section{Cues en overeten in emotionele eters}

Hoofdstukken $\mathbf{2}$ en $\mathbf{3}$ beschrijven studies die als doel hadden de rol van positieve emoties in emotioneel eten te onderzoeken. In beide studies kregen deelnemers filmfragmenten van drie minuten te zien, waarmee ze in een positieve, negatieve of neutrale stemming werden gebracht. Dit werd gevolgd door een zogenaamde smaaktest, waarin de deelnemers dachten dat ze de smaak van verschillende lekkere producten moesten beoordelen, terwijl in het geheim door de onderzoekers werd gemeten hoeveel de deelnemers precies aten. In de eerste studie (Hoofdstuk 2) werd gemeten hoeveel chips en chocolade de deelnemers consumeerden na de stemmingsinductie. In de tweede studie (Hoofdstuk 3) werd gemeten hoeveel de deelnemers dronken van een verse vanille milkshake, zowel tijdens als na het kijken van het filmfragment. Daarnaast werd in de eerste studie een vragenlijst afgenomen om te bepalen of iemand een emotionele eter is, en werd hier in de tweede studie nog een computertaak aan toegevoegd. Deze computertaak, een Impliciete Associatie Test (IAT), meet hoe sterk iemands onbewuste associaties tussen emoties en eten zijn. Zowel in de eerste als de tweede studie bleek dat emotionele eters (deelnemers die het hoogst scoorden op de vragenlijst) niet meer van het voedsel aten na negatieve emoties dan niet-emotionele eters. Ook aten ze in de negatieve stemming niet meer dan in de neutrale stemming. Daarnaast liet de eerste studie zien dat emotionele eters juist meer snacks aten in een positieve stemming vergeleken met een neutrale. In een positieve bui aten de emotionele eters ook meer dan de niet-emotionele eters. De IAT-data uit de tweede studie toonde ook aan dat emotionele eters (deelnemers met sterke impliciete associaties tussen emoties en eten) meer aten wanneer ze in een positieve stemming vergeleken met een negatieve stemming verkeerden, terwijl niet-emotionele eters niet verschilden in hun inname. De studie in Hoofdstuk $\mathbf{2}$ keek ook naar veranderingen in stemming na het eten van lekker voedsel. Emotionele eters geven namelijk vaak aan dat de reden voor het emotieeten is dat ze zich daarna beter voelen; hun negatieve stemming zou dus verminderen. Voor zowel emotionele als niet-emotionele eters werd gevonden dat stemming verbeterde tijdens de eerste vijf minuten van de smaaktest, en gedurende de resterende tien minuten niet meer veranderde. Samengenomen suggereren deze studies dat positieve emoties niet onderschat moeten worden als het gaat om emotioneel eten. In emotionele eters leidden positieve emoties tot hogere voedselinname dan negatieve emoties. 
In Hoofdstuk 4 wordt een derde studie gerapporteerd, waarin verwacht werd dat emotionele eters zich overeten na verschillende cues, niet beperkt tot (negatieve) emoties. Elke deelnemer kwam twee keer naar het lab, en onderging verdeeld over die twee sessies een negatieve stemmingsmanipulatie, een positieve stemmingsmanipulatie, voedsel exposure (het kijken naar en ruiken aan lekker voedsel, zonder ervan te eten) en een controleprocedure waarin ze puzzels maakten. Elke manipulatie werd gevolgd door een smaaktest. Deelnemers werden geclassificeerd als emotionele of niet-emotionele eters aan de hand van zowel vragenlijstscores als hun daadwerkelijke voedselinname tijdens de negatieve stemming. De resultaten ondersteunden voor een groot deel de verwachtingen. Emotionele eters (vastgesteld op basis van zowel vragenlijstscores als echte inname) aten meer dan niet-emotionele eters in alle experimentele condities (negatieve stemming, positieve stemming, voedsel exposure), en ook in de controleconditie. Bovendien aten emotionele eters evenveel in de experimentele condities vergeleken met de controleconditie. Deze bevindingen wijzen erop dat de term 'emotioneel eten' het eetgedrag van de zogenaamde emotionele eters niet afdoende beschrijft: emotionele eters lijken zich immers te overeten na veel meer cues dan alleen (negatieve) emoties. Er wordt voorgesteld dat emotioneel eten wellicht beter'cue-reactief eten' genoemd kan worden.

\section{Individuele verschillen: trait food craving, impulsiviteit, en gewicht}

Hoofdstuk 5 onderzoekt of mensen met hoge trait food craving (een over het algemeen aanwezige sterke zin om te eten), hoge impulsiviteit, en een hoger gewicht sterker reageren op cues die met voedsel te maken hebben dan mensen die deze eigenschappen niet hebben. De voedsel-cues die in deze studie gebruikt werden waren plaatjes van voedsel met veel calorieën en voedsel met weinig calorieën. Obese volwassenen en volwassenen met een gezond gewicht vulden vragenlijsten in om trait craving en impulsiviteit te meten, en deden daarnaast twee computertaken die impulsiviteit vaststellen. Vervolgens deden ze de Visual Search Taak, een computertaak waarmee aandachtsbias gemeten wordt. Specifiek kan deze taak vaststellen of iemands aandacht snel getrokken wordt door de aanwezigheid van voedsel (versnelde detectie van voedsel), en of iemand moeite heeft om zijn of haar aandacht van voedsel af te houden (verhoogde afleiding door voedsel). Zowel versnelde detectie als verhoogde afleiding zijn vormen van aandachtsbias. De resultaten lieten zien dat impulsieve obese mensen sneller hoog-calorisch voedsel opmerkten dan impulsieve mensen met een normaal gewicht. Voor deelnemers laag in impulsiviteit maakte het niet uit of ze obees waren of een normaal gewicht hadden. Die twee groepen verschilden niet in hun aandachtsbias voor voedsel. 


\section{De rol van klassieke conditionering in emotioneel eten}

Hoofdstukken 6 en $\mathbf{7}$ richten zich op twee verschillende manieren waarop conditionering mogelijk betrokken is bij emotioneel eten. De eerste mogelijkheid is dat wanneer mensen in een negatieve stemming zijn, appetitieve conditionering vergemakkelijkt wordt: zij leren de associatie tussen een neutrale cue en lekker eten sneller. Onderzoek heeft laten zien dat eten belonend is en mensen zich beter kan laten voelen, iets dat vooral interessant en relevant is voor mensen die zich negatief voelen. Omdat het voor hen van extra belang is om te leren dat er iets is dat hun negatieve stemming kan verbeteren, wordt gedacht dat zij dit ook sneller leren. Het tweede idee is dat negatieve emoties zich kunnen ontwikkelen tot geconditioneerde cues, en op die manier tot emotioneel eten leiden. In dat geval lokt, nadat conditionering heeft plaatsgevonden, een negatieve emotie automatisch zin om te eten uit.

In Hoofdstuk 6 wordt onderzocht of een negatieve stemming appetitieve conditionering vergemakkelijkt, en of dit beïnvloed wordt door impulsiviteit. De helft van de deelnemers werd in een negatieve stemming gebracht, en de andere helft in een neutrale stemming. Vervolgens ondergingen ze een conditioneringprocedure waarin één specifiek neutraal voorwerp (in dit geval een oranje vaas met witte bloemen) herhaaldelijk werd gepaard met het eten van een hapje chocolademousse, terwijl een gelijkend voorwerp (een groene vaas met rode bloemen) nooit samenging met de chocolademousse. Elke vaas, met of zonder chocolademousse, werd in willekeurige volgorde vier keer aangeboden. Elke keer als de deelnemer de vaas zag, maar voordat ze wel of niet chocolademousse kreeg, werd ze gevraagd aan te geven hoe sterk ze op dat moment verwachtte chocolademousse te krijgen, en hoeveel zin ze had om chocolademousse te eten. Daarnaast werd aan het begin en het einde ook speekselproductie gemeten bij het zien van de vazen, en op het laatste volgde een smaaktest (in aanwezigheid van één van de vazen) om voedselinname te meten. In het begin, als beide vazen nog neutraal zijn, zijn verwachting en zin om te eten bij beide vazen hetzelfde. Conditionering vindt plaats als de vaas die met eten gepaard gaat na verloop van tijd tot hogere verwachting en zin om te eten leidt dan de vaas die niet met eten gepaard gaat. Voor speeksel en inname geldt hetzelfde; als de vaas die samen is gegaan met chocolademousse meer speeksel en meer inname veroorzaakt dan de vaas die niet met de mousse is samen gegaan, is er sprake van succesvolle conditionering. De resultaten lieten zien dat appetitieve conditionering inderdaad plaats vond. Zowel verwachting als zin nam toe bij de ene vaas, en af bij de andere vaas. Het verwachte verschil tussen mensen in een negatieve en neutrale stemming werd echter niet gevonden. In beide groepen namen verwachting en zin in dezelfde mate toe of af. Een negatieve stemming vergemakkelijkt 
conditionering dus niet. Een effect van impulsiviteit werd alleen gevonden bij de smaaktest. Hoog impulsieve mensen aten minder chocolademousse in aanwezigheid van de vaas die eerder telkens met de mousse gepaard was dan in aanwezigheid van de vaas die niet met de mousse gepaard was.

Hoofdstuk $\mathbf{7}$ doet verslag van de mogelijkheid die veronderstelt dat negatieve emoties kunnen veranderen in geconditioneerde stimuli en zo voorspellers van voedselinname worden. Deelnemers ondergingen een conditioneringprocedure waarin ze tien verschillende stimuli te zien kregen. Vijf hiervan wekten negatieve gevoelens op bij de deelnemers, de andere vijf neutrale gevoelens. De deelnemers werden ook willekeurig verdeeld over twee groepen. De ene groep kreeg telkens een chocolaatje te eten als ze negatieve gevoelens ervoeren (FoodNeg groep), en de andere groep kreeg alleen een chocolaatje bij neutrale gevoelens (FoodNeu groep). Net als in de vorige studie werden verwachting om te eten, zin om te eten, en speekselproductie gemeten. Daarnaast moesten deelnemers vooraf en achteraf aangeven hoe onaangenaam ze het vonden om negatieve emoties te ervaren, en kregen ze op het laatst een chocolade vs. geld keuzetaak terwijl ze zich negatief voelden. De voorspelling was dat deelnemers voor wie negatieve emoties gepaard gingen met chocolade deze emoties achteraf als minder onplezierig zouden beoordelen. Voor de keuzetaak werd gedacht dat de groep die tijdens de conditioneringprocedure geleerd had dat negatieve gevoelens chocolade voorspelden, chocolade boven geld zouden verkiezen. De andere groep (die geen verband geleerd had tussen negatieve emoties en chocolade) zou beide opties even vaak moeten kiezen. Uit de data met betrekking tot verwachting en zin om te eten bleek dat conditionering succesvol was en dat negatieve gevoelens zich kunnen ontwikkelen tot geconditioneerde stimuli. Deelnemers die chocolade kregen bij het ervaren van negatieve emoties gaven aan dat ze een hogere verwachting en meer zin hadden om te eten als ze zich negatief voelden dan wanneer ze in een neutrale stemming waren. Zoals verwacht lieten deelnemers uit de FoodNeg-groep ook een sterkere voorkeur voor chocolade dan geld zien tijdens de keuzetaak, maar dat gold alleen voor de deelnemers met een relatief hoog BMI. Daarnaast bleek dat in de loop van het experiment niet-emotionele eters niet veranderen in hoe onaangenaam ze het ervaren van negatieve emoties vonden. Zij gaven vooraf en achteraf hetzelfde antwoord. Bij emotionele eters was dat heel anders. Zij gaven aan het eind van het onderzoek aan dat ze het ervaren van negatieve emoties vervelender waren gaan vinden, tenzij die negatieve emoties gepaard gingen met het eten van chocolade. In dat geval was er juist een vermindering in hoe onaangenaam ze die emoties vonden. Het lijkt er dus op dat in emotionele eters het eten van lekker voedsel tijdens negatieve momenten de effecten ervan verzacht: het maakt die 
negatieve momenten minder erg. Op basis van de resultaten van beide studies lijkt het waarschijnlijk dat klassieke conditionering een rol speelt bij emotioneel eten.

Hoofdstuk 8 presenteert een discussie en conclusie met betrekking tot elk van de drie hoofdpunten zoals weergegeven aan het begin van deze samenvatting. De eerste conclusie is dat emotionele eters niet specifiek overeten als reactie op negatieve emoties, maar ook na het ervaren van positieve emoties en verschillende andere cues. De tweede conclusie is dat het aannemelijk is dat klassieke conditionering kan leiden tot emotioneel eten. Ten derde kunnen individuele verschillen zowel cue reactiviteit als leren beïnvloeden. Het hoofdstuk bevat tevens aanbevelingen voor toekomstig onderzoek. Daarnaast worden methodologische overwegingen betreffende de studies gegeven, en wordt aandacht besteed aan de klinische implicaties van de bevindingen. 


Valorization Addendum 

This valorization addendum highlights the significance of the studies presented in this dissertation. This is done by outlining the way in which our studies are relevant for society, by explaining which target groups will benefit from the results, and by providing future possibilities in terms of activities and products resulting from the findings. In addition, the innovative aspects of the current studies are discussed. Finally, manners in which knowledge resulting from the studies has been disseminated are given, and ideas and plans for future knowledge dissemination are made.

\section{Relevance of the studies}

It is no secret that obesity is one of the biggest health issues of our time. In the last decades, the prevalence of obesity and overweight has risen steadily, and the turning point has yet to come. As we speak, approximately $70 \%$ of the adult population in the US is overweight or obese (Ogden, Carroll, Kit, \& Flegal, 2014). In the Netherlands, this number is close to 50\% (Health Monitor, 2012). Obesity is related to serious physical and psychosocial health concerns, and has a significant societal and economical impact. Clearly, measures need to be taken not only to stop the obesity-epidemic from rising any further, but also to knock down the current figures. To mitigate current obesity levels, research into all factors that contribute to excessive weight gain is of utmost importance. One of the most obvious factors leading to overweight and obesity is overeating, of which emotional eating is considered a specific case. To be able to address and treat emotional eating correctly, it is crucial that the phenomenon is correctly understood. Questions that therefore need to be answered include which emotions elicit overeating, whether certain individual characteristics can make one more susceptible to emotional eating, and how emotional eating develops and is maintained. The studies described in this thesis address these questions. 
Chapters 2, 3 and 4 shed light on the exact cues that elicit overeating in emotional eating. Chapter 5, although not focused on emotional eating per se, investigates the influence of weight status, impulsivity and trait craving on how individuals respond to food cues. Chapters 6 and 7 focus on classical conditioning as a developmental or maintenance factor in emotional eating.

\section{Who benefits from the findings?}

The findings as discussed in this dissertation are relevant to three distinct target groups: researchers, health care professionals, and individuals looking for assistance in controlling their (emotional) eating behaviour. Researchers with an interest in emotional eating benefit from the current studies in that these studies add new information to the existing knowledge regarding emotional eating, for example in terms of the importance of positive emotions and the role of classical conditioning. Although this dissertation does not lead to a full and complete understanding of emotional eating, the studies do contribute to the field, and fellow researchers could build on the used paradigms and obtained findings when designing new studies on this topic. Health care professionals could use the results from the studies when treating (overweight) individuals who engage in emotional eating. The results from the first three chapters stress that treatment should likely not only focus on negative emotions, but also on positive emotions and perhaps even other cues that lead to overeating. The final two chapters introduce classical conditioning as a mechanism in emotional eating. Knowledge about factors that lead to or maintain emotional eating will enable therapists to adjust treatment to be more effective. The final target group, individuals who engage in emotional eating, can benefit from the findings in the sense that a full understanding of the concept of emotional eating can, in the end, lead to purposeful and effective treatment of this type of overeating. A reduction of overeating would in turn lead to weight loss and a decrease in both the physical and mental health problems associated with overweight and obesity.

\section{Activities and products}

In general, only a small part of scientific research can be immediately translated into tangible activities, products, processes or services. Mostly, however, this is a much slower process, with many studies making small but essential contributions to a larger whole. The same is true for the studies in this dissertation: they all contribute to a better understanding of emotional eating. Importantly, the better emotional eating is understood, the better preventions or interventions aimed at this phenomenon can be. Although the current 
studies might not lend themselves for direct translation to activities and products, they are essential to the optimal design of activities, products, processes and services in the future.

One type of activity or product that could eventually be derived from the present results relates to therapeutic strategies. Based on the classical conditioning principle of extinction, cue exposure with response prevention (CERP; see chapter 8 of this dissertation for a more detailed description) has been developed as a treatment strategy aimed at reducing food intake. In CERP, the association between a cue that signals food on the one hand and actual food intake on the other hand is overruled by a stronger cue - no food intake association. This is accomplished through repeated presentation of the food-cue without it being followed by eating. CERP has been shown to be effective in reducing overeating in various populations, such as overweight adults, overweight children, and patients suffering from Bulimia Nervosa (Boutelle, et al., 2011; Jansen, Broekmate, \& Heymans, 1992; Martinez-Mallén, et al., 2007; Schyns, Roefs, Mulkens, \& Jansen, 2015; Toro, et al., 2003). The cues commonly used in these studies are the sight and smell of palatable food. Although CERP has not yet been tested specifically with regard to emotional cues, it is likely to have potential for reducing emotion-induced overeating. Upon positive evaluation of CERP with emotional cues in studies involving clinical samples, this strategy could be implemented by therapists in the treatment of emotional eating.

\section{Innovation}

The studies presented in this dissertation are innovative in several ways. Chapters 2-4 challenge the prevailing assumption that emotional eating reflects overeating in response to specifically negative emotions. In addition, chapters 3 and 4 go beyond the traditional identification of emotional eaters based on questionnaire scores (whose validity is debatable) by also classifying individuals as emotional eaters depending on their performance on an IAT and on their actual food intake when feeling negative. Although these classification measures are in need of more research, they do hold high face validity. Chapter 5 was the first study to investigate the combined effects of weight status and impulsivity on attention bias. Chapters 6 and 7 are highly innovative because they are among the few studies that do not merely describe food consumption of emotional eaters (i.e., changes in intake in response to the experience of specific emotions), but are instead aimed at the mechanisms underlying emotional eating. More specifically, these chapters experimentally test two different pathways through which classical conditioning could serve as an aetiological and/ or maintaining factor in emotional eating. Although classical conditioning has been previ- 
ously suggested to be involved in emotional eating (Jansen, 1998; Jansen, Havermans, \& Nederkoorn, 2011; Wardle, 1990), these studies are the first to put this hypothesis to the test.

\section{Planning and implementation}

Concerning activities, products, processes or services, we intend to conduct studies on extinction and CERP for emotional cues in both student and clinical samples. These studies are the next step in testing the suitability of CERP as a treatment technique for individuals who engage in emotional eating.

With regard to knowledge dissemination, we have undertaken various efforts to ensure that the knowledge obtained from our studies reached the three target groups. To spread the findings of the studies to the international research community, we have published papers in several international journals. In addition, we have presented all the findings at international conferences, which are frequented by researchers from all over the world. Findings have also been published in Dutch journals and presented at Dutch conferences. Through these Dutch channels results have not only reached Dutch scientists, but also a broader audience of mental health care professionals. Some of the studies, particularly chapter 2 and chapter 3, have been picked up by the international media. This ensured dissemination of the research findings to the general public. The studies were for example featured on medicaldaily.com (a US-based medical news website), artikalmagazin.de (a German online magazine), spirehealthcare.com (the second largest provider of private healthcare in the UK), and menshealth.com (the US website of the popular and well-known Men's Health magazine). Furthermore, the studies were given more national attention through an interview for a youth version of the Dutch magazine GezondNU.

In the future, we plan to continue disseminating our research by means of national and international journal publications, national and international conference visits, and bringing the findings to the attention of the general public by using traditional and social media. The latter will be achieved through for example press releases, media interviews, and blog posts. I have followed a course on 'scientific writing for a broad audience', which was aimed at helping scientists to translate their scientific findings into interesting and easy-to-understand articles intended for the lay public. 


\section{References}

Boutelle, K. N., Zucker, N. L., Peterson, C. B., Rydell, S. A., Cafri, G., \& Harnack, L. (2011). Two novel treatments to reduce overeating in overweight children: a randomized controlled trial. Journal of consulting and clinical psychology, 79(6), 759.

Health Monitor 2012 [Gezondheidsmonitor GGD'en, CBS en RIVM, 2012. In Dutch]. WWW Document (2013, September 12). Statistics Netherlands. Retrieved from http://www.cbs.nl/nl- NL/menu/ themas/gezondheidwelzijn/cijfers/incidenteel/maatwerk/2013- gezondheidsmonitor2012-mw.htm. Accessed March 2015.

Jansen, A. (1998). A learning model of binge eating: cue reactivity and cue exposure. Behaviour research and therapy, 36(3), 257-272.

Jansen, A., Broekmate, J., \& Heymans, M. (1992). Cue-exposure vs self-control in the treatment of binge eating: a pilot study. Behaviour Research and Therapy, 30(3), 235-241.

Jansen, A., Havermans, R. C., \& Nederkoorn, C. (2011). Cued overeating Handbook of Behavior, Food and Nutrition (pp. 1431-1443): Springer.

Martinez-Mallén, E., Castro-Fornieles, J., Lázaro, L., Moreno, E., Morer, A., Font, E., et al. (2007). Cue exposure in the treatment of resistant adolescent bulimia nervosa. International Journal of Eating Disorders, 40(7), 596-601.

Ogden, C. L., Carroll, M. D., Kit, B. K., \& Flegal, K. M. (2014). Prevalence of childhood and adult obesity in the united states, 2011-2012. JAMA, 311(8), 806-814.

Schyns, G., Roefs, A., Mulkens, S., \& Jansen, A. (2015). Expectancy violation, reduction of food cue reactivity and less eating in the absence of hunger after one food cue exposure session for overweight and obese women. Manuscript submitted for publication.

Toro, J., Cervera, M., Feliu, M., Garriga, N., Jou, M., Martinez, E., et al. (2003). Cue exposure in the treatment of resistant bulimia nervosa. International Journal of Eating Disorders, 34(2), 227-234

Wardle, J. (1990). Conditioning processes and cue exposure in the modification of excessive eating. Addictive Behaviors, 15(4), 387-393 

Dankwoord 

Het boek is af! Het moge duidelijk zijn dat het doorlopen van een promotietraject en het schrijven van een proefschrift niet iets is dat je in je eentje kunt doen. Hulp komt van vele kanten. Hier neem ik graag de gelegenheid om aan iedereen die mij in de afgelopen vier jaar op wat voor manier dan ook geholpen heeft een bedankje te schrijven.

Anita, op het moment van mijn promotie is het zo'n tien jaar geleden dat ik voor het eerst bij je aanklopte. Als masterstudent was ik op zoek naar een begeleider voor mijn onderzoeksstage, en zo zette ik mijn eerste stap in de wereld van emotionele eters. Na die eerste stage heb ik nog vele malen onder je supervisie gewerkt, als stagiaire in de research master, als student-assistent, onderzoeksassistent, promovendus, en uiteindelijk als post-doc. Ik wil je ontzettend bedanken voor alle kansen en mogelijkheden die je me in de afgelopen jaren hebt gegeven. Ik heb ontzettend veel van je geleerd, en doe dat nog steeds. Ik bewonder je als wetenschapper en als persoon, en waardeer het enorm dat je altijd tijd voor me had als het nodig was, maar ook genoeg vertrouwen in me had om me op andere momenten mijn gang te laten gaan. Ook je uitgebreide feedback op mijn stukken is erg waardevol geweest. Ik kijk uit naar de komende twee jaar!

Remco, je werd mijn co-promotor toen het project al een tijdje liep, en dat kwam precies op het juiste moment. Je kennis, ervaring en kritische onderzoeksblik hebben me veel geholpen bij het opzetten van studies en het schrijven en herschrijven van artikelen. Je optimisme en positiviteit waren erg welkom tijdens momenten van stress. Dank! 
Onderzoek is niet mogelijk zonder proefpersonen. Via deze weg bedank ik iedereen die in de afgelopen jaren aan mijn studies heeft deelgenomen. Ook grote dank aan Anastacia en Angeliki-Maria voor hun hulp bij het werven en testen van proefpersonen.

Dank aan mijn co-auteurs, Anita, Remco, Anne, Sjaan, Katrijn, Karolien, Anastacia, Elsmarieke, Jan, en Wim voor jullie betrokkenheid en hulp in de vorm van studiedesign, data verzameling, data analyses, en feedback.

Leden van de beoordelingscommissie, prof. dr. Madelon Peters, prof. dr. Marcus Huibers, dr. Carolien Martijn, prof. dr. Denise de Ridder en dr. Tom Smeets, hartelijk dank voor het lezen en beoordelen van mijn proefschrift. Dank aan prof. dr. Eric van Furth en prof. dr. Rob Markus voor het plaatsnemen in mijn promotiecommissie.

Technische en praktische ondersteuning is onmisbaar. Jacco en Erik, bedankt voor jullie hulp bij het programmeren en maken van computertaakjes en ondersteuning bij labproblemen. Jessie, Marionne, Truus en Lindy, dank jullie wel voor alle praktische hulp in de afgelopen jaren, die jullie zonder uitzondering gegeven hebben met veel geduld en een grote lach.

Veel dank aan al mijn huidige en voormalige collega's van de eetgroep: Anita, Carolien, Sjaan, Anne, Remco, Sandra, Katrijn, Eva, Janneke, Lotte, Nele, Jessica A, Karolien, Ghislaine, Bastiaan, Fania, Sieske, Eric, Valerie, Ramona, Astrid, Haris, Jessica W, en Clare. Het is geweldig om van deze groep fantastische onderzoekers deel uit te mogen maken, en ik dank jullie voor alle gezellige momenten en waardevolle feedback die jullie me gegeven hebben.

Karolien, Janneke, Jessica W, en Conny, mijn kamergenootjes door de jaren heen. Altijd hadden jullie tijd voor een gezellig gesprek, het geven van advies, of het uitwisselen van frustraties. Bedankt!

Speciale dank aan mijn mede-aio's van CPS en daarbuiten, maar in het bijzonder Rena, Jessica, Kai, Bastiaan, Karolien, Ghislaine, Fania en Sieske voor alle gezelligheid tijdens congressen, EPP-symposia, treinritten, spellenavonden, etentjes, koffiepauzes, en gewoon tussendoor. Promoveren is niet alleen maar hard werken, en ik ben blij met alle leuke en ontspannende momenten die ik dankzij jullie heb gehad. 
Rena en Maurice, Jessica en Kai, jullie zijn mijn 'Maastricht-mensen'. Ik hoop dat we nog heel vaak samen koffie drinken, lekker eten, stitch 'n bitchen, uren volpraten met zin en onzin, spelletjes spelen, en andere leuke dingen doen. Zonder jullie zou Maastricht maar half zo leuk zijn!

Rena en Jessica, mijn paranimfen. Dank jullie wel dat jullie op die o zo belangrijke dag achter me staan. Rena, we met at the start of the research master in 2008, and became friends fast. From devouring Andy Field together to being a guest at your Greek wedding and going through our PhD's almost simultaneously (fortunately Maastricht and Leuven aren't too far away from each other); we have made some wonderful memories! You're an amazing person, and I hope we continue our get-togethers with chats, crochet and balcony (or garden!) dinners until we're wrinkled and grey. Jessica, het was fantastisch om de afgelopen vier jaar samen met jou te doorlopen. Zelfs ons proefschrift schrijven begonnen we op exact dezelfde dag. Ik heb genoten van al onze koffiepausjes, of die nu waren om even lekker te klagen, onze publicaties te vieren, of gewoon bij te kletsen. Ik ben blij dat ik je behalve collega ook vriendin kan noemen, en ik verheug me op het komende jaar waarin we nog samenwerken. Mochten onze wegen zich daarna scheiden, dan is dat toch zeer zeker alleen op werkgebied!

Familie, schoonfamilie en vrienden, ik heb niet de ruimte om jullie allemaal bij naam te noemen, maar ik wil jullie stuk voor stuk bedanken voor de interesse die jullie vanaf dag één hebben getoond in mijn onderzoek en proefschrift. Ik vind het tof dat ik jullie nu eindelijk het resultaat kan laten zien!

Lieve Shanna, zusjes zijn bijzonder. Het is zo fijn om te weten dat wij er altijd en onvoorwaardelijk voor elkaar zijn, en dat er niets is dat daar tussen kan komen. Ik ben blij en trots dat jij mijn zusje bent!

Lieve pap en mam, jullie bedanken in een paar regels is eigenlijk niet te doen. Ik kan altijd bij jullie terecht, jullie staan altijd voor me klaar, en niets is ooit teveel. Zoveel liefde, steun, trots en vertrouwen zijn niet voor iedereen vanzelfsprekend, en Shanna en ik hadden het ons niet beter kunnen wensen. Dank jullie wel. Voor alles.

Lieve Rob, it's you I fell into! Wat een geluk heb ik om elke dag thuis te komen bij iemand die me opvrolijkt als het even tegenzit, en die mee juicht als er iets te vieren valt. Je bent mijn maatje, mijn beste vriend. Waar we ook zijn en wat we ook doen, met jou kan ik de hele wereld aan. 

Curriculum Vitae 

Peggy Margaretha Henrica Bongers was born on March 27, 1984, in Panningen, The Netherlands. She graduated from secondary school (VWO, Bouwens van der Boijecollege, Panningen) in 2002 and continued her education studying psychology at the Faculty of Psychology and Neuroscience at Maastricht University. She obtained her bachelor's degree in 2005 and her master's degree (Experimental Health Psychology - cum laude) in 2006. After a year of travelling around Australia, New Zealand and South-East Asia (2007/2008), she returned to Maastricht to start the two-year research master Cognitive Neuroscience, Neuropsychology and Psychopathology (Psychopathology track). During this time she did a clinical internship at U-Center in Epen, a private clinic specialized in the treatment of burnout, depression and addiction. In addition, over the course of the research master, she held several positions as a student research assistant. She received her second master's degree cum laude in 2010. After working as a research assistant for ten months, she started her PhD-project at the Department of Clinical Psychological Science at Maastricht University in June 2011. During her PhD, she obtained her BKO (Basis Kwalificatie Onderwijs / University Teaching Qualification). Since July 2015 she works as a postdoctoral fellow at the Department of Clinical Psychological Science, Maastricht University. 



\section{PUBLICATIONS}

\section{Journal articles}

Bongers, P., de Graaff, A., \& Jansen, A. (in press). 'Emotional' does not even start to cover it: Generalization of overeating in emotional eaters. Appetite.

Bongers, P., \& Jansen, A. (in press). Emotional eating and Pavlovian learning: Evidence for conditioned appetitive responding to negative emotional states. Cognition and Emotion.

Bongers, P., van den Akker, K., Havermans, R., \& Jansen, A. (2015). Emotional eating and Pavlovian learning: Does negative mood facilitate appetitive conditioning? Appetite, 89, 226-236.

Bongers, P., van de Giessen, E., Roefs, A., Nederkoorn, C., Booij, J., van den Brink, W., \& Jansen, A. (2015). Being impulsive and obese increases susceptibility to speeded detection of highcalorie foods. Health Psychology, 34, 677-685.

Bongers, P. (2014). De emotionele eter bestaat niet. De Psycholoog, 49, 28-33.

Bongers, P., Jansen, A., Houben, K., \& Roefs, A. (2013). Happy eating: The Single-Target Implicit Association Test predicts overeating after positive emotions. Eating Behaviors, 14, 348-355.

Bongers, P., Jansen, A., Havermans, R., Roefs, A., \& Nederkoorn, C. (2013) Happy eating: The underestimated role of overeating in a positive mood. Appetite, 67, 74-80.

Papachristou, H., Nederkoorn, C., Havermans, R., Bongers, P., Beunen, S., \& Jansen, A. (2013). Higher levels of trait impulsiveness and a less effective response inhibition are linked to more intense cue-elicited craving for alcohol in alcohol-dependent patients. Psychopharmacology, 228, 641-649.

Bongers, P., Jansen, A., Papachristou, H., Nederkoorn, C., \& Havermans, R. (2012). Over de te vroege teloorgang van exposuretherapie voor verslaafden. Wat is een goede exposure? Directieve Therapie, 3, 199-211. 
Jansen, A., Nederkoorn, C., Roefs, A., Bongers, P., Teugels, T., \& Havermans, R. (2011). The proof of the pudding is in the eating: Is the DEBQ - External Eating Scale a valid measure of external eating? International Journal of Eating Disorders, 44, 164-168.

\section{Conference presentations}

Bongers, P., \& Jansen, A. (2015). Emotioneel eten is eenvoudig aan te leren. Paper presented at the Najaarscongres van de Vereniging voor Gedragstherapie en Cognitieve Therapie, Veldhoven, The Netherlands.

Bongers, P., Havermans, R., \& Jansen, A. (2015). Emotional eating and Pavlovian learning: Can negative emotions become conditioned stimuli? Poster presented at the annual meeting of the British Feeding and Drinking Group, Wageningen, The Netherlands.

Bongers, P., Jansen. A., van den Akker, K., \& Havermans, R. (2014). Emotional eating: A matter of learning? Paper presented at the annual meeting of the British Feeding and Drinking Group, Portsmouth, United Kingdom.

Bongers, P., Jansen. A., van den Akker, K., \& Havermans, R. (2014). Emotioneel eten: Een kwestie van leren? Paper presented at the Najaarscongres van de Vereniging voor Gedragstherapie en Cognitieve Therapie, Veldhoven, The Netherlands.

Bongers, P., Jansen, A., Havermans, R., Nederkoorn, C., Houben, K., \& Roefs, A. (2013). Happy Eating: the role of positive mood in emotional eating. Poster presented at the annual Meeting of the British Feeding and Drinking Group, Loughborough, United Kingdom.

Bongers, P., Jansen, A., Houben, K., \& Roefs, A. (2012). The IAT as a measure of emotional eating. Paper presented at the annual meeting of the British Feeding and Drinking Group, Brighton, United Kingdom. 

